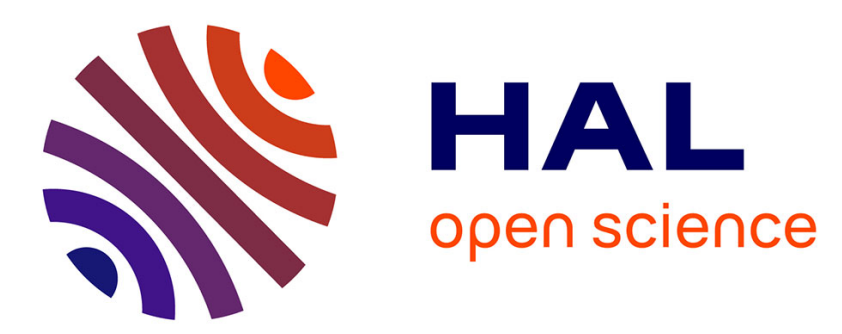

\title{
High resolution IR spectrum of the $\nu 8$ band of CH2DF: analysis of the interactions with the near vibrational levels
}

\author{
Raffaella Visinoni, Agostino Baldacci, Giandomenico Nivellini
}

\section{- To cite this version:}

Raffaella Visinoni, Agostino Baldacci, Giandomenico Nivellini. High resolution IR spectrum of the $\nu 8$ band of CH2DF: analysis of the interactions with the near vibrational levels. Molecular Physics, 2008, 106 (21-23), pp.2409-2415. 10.1080/00268970802502519 . hal-00513231

\section{HAL Id: hal-00513231 \\ https://hal.science/hal-00513231}

Submitted on 1 Sep 2010

HAL is a multi-disciplinary open access archive for the deposit and dissemination of scientific research documents, whether they are published or not. The documents may come from teaching and research institutions in France or abroad, or from public or private research centers.
L'archive ouverte pluridisciplinaire HAL, est destinée au dépôt et à la diffusion de documents scientifiques de niveau recherche, publiés ou non, émanant des établissements d'enseignement et de recherche français ou étrangers, des laboratoires publics ou privés. 


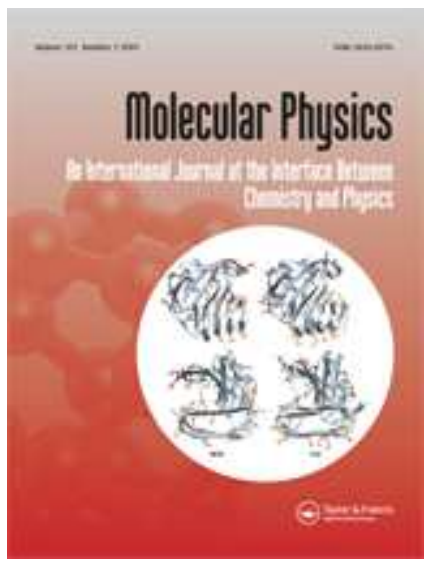

High resolution IR spectrum of the $\mathrm{v}_{8}$ band of $\mathrm{CH}_{2}$ DF: analysis of the interactions with the near vibrational levels

\begin{tabular}{|c|c|}
\hline Journal: & Molecular Physics \\
\hline Manuscript ID: & TMPH-2008-0211.R1 \\
\hline Manuscript Type: & Full Paper \\
\hline $\begin{array}{r}\text { Date Submitted by the } \\
\text { Author: }\end{array}$ & 18-Sep-2008 \\
\hline Complete List of Authors: & $\begin{array}{l}\text { Visinoni, Raffaella; Università Ca' Foscari di Venezia, Dipartimento } \\
\text { di Chimica Fisica } \\
\text { BALDACCI, Agostino; Università Ca' Foscari di Venezia } \\
\text { Nivellini, GianDomenico; Università di Bologna, Dipartimento di } \\
\text { Chimica Fisica e Inorganica }\end{array}$ \\
\hline Keywords: & $\begin{array}{l}\text { monodeutero methyl fluoride, Fourier transform spectroscopy, } \\
\text { rovibrational parameters, Fermi and Coriolis resonances }\end{array}$ \\
\hline
\end{tabular}

\section{s ScholarONE" \\ Manuscript Central}


High resolution IR spectrum of the $v_{8}$ band of $\mathrm{CH}_{2} \mathrm{DF}$ : analysis of the interactions with the near vibrational levels

\author{
R. Visinoni, A. Baldacci \\ Università $\mathrm{Ca}^{\prime}$ Foscari di Venezia \\ Dipartimento di Chimica Fisica \\ D. D. 2137, I-30123 Venezia, Italy \\ and \\ G. Nivellini \\ Università di Bologna \\ Dipartimento di Chimica Fisica e Inorganica \\ Viale del Risorgimento 4, I-40136 Bologna, Italy
}

Running title: The $v_{8}$ band of $\mathrm{CH}_{2} \mathrm{DF}$

Corresponding author:

Dr. R. Visinoni

tel: +39-0412348526, fax: +39-0412348594

e-mail: visinon@unive.it

No. of pages: 10

No. of figures: 5

No. of tables: 3 


\begin{abstract}
The IR spectrum of the $v_{8}$ band of monodeutero methyl fluoride has been recorded with a resolution of $0.004 \mathrm{~cm}^{-1}$ using a Fourier transform spectrometer. The $v_{8}$ vibration, of $\mathrm{A}^{\prime \prime}$ symmetry species, gives rise to a pure $c$-type envelope centred at $1298.58 \mathrm{~cm}^{-1}$, resembling a perpendicular band of a prolate symmetric rotor. From the spectral analysis it has been found that $v_{8}$ is coupled with $v_{4}$ for the low $K_{a}$ sublevels while for the higher ones other vibrational states, directly or indirectly, are also interacting. For a satisfactory reproduction of the rovibrational data of $v_{8}$, the Watson's Hamiltonian in the $\mathrm{I}^{\mathrm{r}}$ representation has been implemented with interaction parameters which included first and second order $a$-, $b$-type and $c$-type Coriolis couplings with $v_{4}$ and $v_{9}$, respectively. Being these states also interacting with other levels, the effective spectroscopic parameters of the analyzed band up to fourth order have been obtained from a data set formed by the six fundamentals located below $1500 \mathrm{~cm}^{-1}$.
\end{abstract}




\section{Introduction}

Accurate spectroscopic constants on methyl fluoride and its isotopologues are of considerable importance for the determination of the equilibrium structure [1], ab initio cubic force field [2] and anharmonic potential energy surface [3]. Among the different isotopologues of this molecule, $\mathrm{CH}_{2} \mathrm{DF}$ is the least characterized. Low resolution IR analysis, aimed at the identification of the fundamental bands, was carried out long time ago [4]. In the first high resolution paper [5] the $v_{5}$ and $v_{6}$ fundamentals were analyzed as isolated bands, by fixing the ground state constants to the microwave values obtained by Clark and De Lucia [6]. Subsequently, the $v_{3}$ and $v_{4}$ vibrations were studied using a dyad model [7]; improved ground state parameters were also obtained from a combined fit of infrared ground state combination differences (GSCDs) and microwave data of ref. [6]. The anomalous structure of the strongly perturbed $v_{9}$ was then investigated [8] and its interpretation required the re-analysis of the $v_{5}$ and $v_{6}$ states, which are also mutually interacting. The present contribution aims at supplying additional information on $\mathrm{CH}_{2} \mathrm{DF}$ through the rovibrational analysis of the $v_{8}$ vibration, located at $1298.58 \mathrm{~cm}^{-1}$. The rotational structure of this perturbed band has been characterized in detail. In order to obtain a satisfactory set of upper state constants, a simultaneous treatment of $v_{8}$ with the near vibrational levels was needed. The model, implemented step by step with interacting terms, required in the final form the employment of experimental data arising from the six lower fundamental bands, located below $1500 \mathrm{~cm}^{-1}$.

\section{Experimental}

The preparation of $\mathrm{CH}_{2} \mathrm{DF}$ was accomplished using the procedure adopted by Edgel and Parts [9] for the synthesis of alkyl and isotopically substituted alkyl fluorides. The precursor was the monodeuterated methyl alcohol (Aldrich, 99\%). The purity of the obtained compound, higher than $95 \%$, was tested by gas-chromatography; the gas-phase infrared spectrum showed weak absorptions due to the by-product of synthesis $\mathrm{CH}_{3} \mathrm{~F}$. The high resolution $\left(0.004 \mathrm{~cm}^{-1}\right.$, unapodized) spectrum was recorded at the University of Bologna using a Bomen DA3.002 Fourier transform infrared spectrometer. The instrument was equipped with a globar source, a $\mathrm{KBr}$ beam splitter and a high-sensitivity mercury-cadmium-tellurium (MCT) semiconductor 
as detector. A multipass absorption cell with a base length of $25 \mathrm{~cm}$, by Infrared Analysis, Inc., was used with the optical path length set at $5 \mathrm{~m}$. The spectrum was obtained at room temperature with a sample pressure of about $67 \mathrm{~Pa}$ for a negligible pressure broadening $\cong$ $0.0004 \mathrm{~cm}^{-1}$ ). A total of 800 scans were co-added for a satisfactory signal-to-noise ratio. No apodization function was applied. The resulting line width of unblended lines was about 0.005 $\mathrm{cm}^{-1}$. The calculated Doppler line width (FWHM) in the $v_{8}$ band region is $0.0027 \mathrm{~cm}^{-1}$. The weak lines of $\mathrm{CH}_{3} \mathrm{~F}$ were used as internal standard to calibrate the infrared spectrum [10].

\section{Analysis of the spectrum}

Monodeutero methyl fluoride is a near prolate asymmetric top of $\mathrm{C}_{\mathrm{s}}$ symmetry with the asymmetry parameter $\kappa=-0.978$. The $v_{8}$ mode, described as $\mathrm{CD}$ out-plane-bend $/ \mathrm{CH}_{2}$ rock [5], is of $\mathrm{A}^{\prime \prime}$ symmetry species and gives rise to a $c$-type band of medium intensity centred at $1298.58 \mathrm{~cm}^{-1}$. The low resolution infrared spectrum of this band, depicted in Figure 1, shows the $\mathrm{R}$ branch partially overlapped by the $\mathrm{P}$ branch of the weaker $v_{4}$ at $1364.17 \mathrm{~cm}^{-1}$. The prominent clusters in the $\mathrm{P}$ and $\mathrm{R}$ branches are due to the unresolved ${ }^{\mathrm{P}, \mathrm{R}} \mathrm{Q}_{\mathrm{K}}(\mathrm{J})$ lines. They were easily identified being the broad and sharp peaks near the band centre typical of the ${ }^{\mathrm{P}} \mathrm{Q}_{1}(\mathrm{~J})$ and ${ }^{\mathrm{R}} \mathrm{Q}_{0}(\mathrm{~J})$ multiplets respectively. The manifolds corresponding to ${ }^{\mathrm{P}} \mathrm{Q}_{2}(\mathrm{~J})$ and ${ }^{\mathrm{R}} \mathrm{Q}_{1}(\mathrm{~J})$ are less pronounced since influenced by the most significant effect of asymmetry splitting.

The assignment of the high resolution spectrum proceeded in a straightforward way. The $\mathrm{J}$ line sequence belonging to the strong ${ }^{\mathrm{R}} \mathrm{Q}_{0}(\mathrm{~J})$ and ${ }^{\mathrm{P}} \mathrm{Q}_{1}(\mathrm{~J})$ multiplets was readily checked through GSCDs using the ${ }^{\mathrm{P}} \mathrm{P}_{1,2}(\mathrm{~J})$ series of transitions. The details of the rotational structure of the sharp ${ }^{\mathrm{R}} \mathrm{Q}_{0}(\mathrm{~J})$ group of Figure 1 are depicted in Figure 2, where some unlabelled features belong to the weaker ${ }^{\mathrm{R}} \mathrm{P}_{\mathrm{K}}(\mathrm{J})$ and ${ }^{\mathrm{P}} \mathrm{R}_{\mathrm{K}}(\mathrm{J})$ subbranches. To be noted that the sequence degrades to lower wavenumbers up to $\mathrm{J}=21$ and then follows an opposite trend. From the assigned transitions a set of preliminary upper state parameters was determined and employed to predict further subbands through a bootstrap procedure. This was done with a semiautomatic program based on correlation plots accounting for wavenumber values and relative intensity of the lines, early proposed by Nakagawa and Overend [11]. 
In the subbands with $\mathrm{K}_{\mathrm{a}}{ }^{\prime}>4$ the structure approaches that of a perpendicular band of a symmetric top, as clearly observable in the P-branch region where the interference with lines due to $v_{4}$ is greatly reduced. Concerning the ${ }^{P} P_{K}(J)$ and ${ }^{R} R_{K}(J)$ subbranches the distinct lines, spreading over a wide range, are regularly spaced by $2 \bar{B} \cong 1.56 \mathrm{~cm}^{-1}$. In the ${ }^{\mathrm{P}, \mathrm{R}} \mathrm{Q}_{\mathrm{K}}(\mathrm{J})$ multiplets the $\mathrm{J}$ progression degrades towards higher wavenumbers, indicating that the coefficient $\left(\alpha^{\mathrm{A}}-\alpha^{\mathrm{B}}\right)$ is positive. A typical example is given in Figure 3, where the details of the ${ }^{\mathrm{P}} \mathrm{Q}_{7}(\mathrm{~J})$ manifold are shown. In the same figure it is worth noting the effect of a local interaction splitting the even and odd components of $\mathrm{K}_{\mathrm{a}}^{\prime}=6$, otherwise degenerate. The crossings occur at $\mathrm{J}=24 / 25$ even and $\mathrm{J}=25 / 26$ odd components. The experimental shifts from the predicted line positions, before and after the crossings, suggested that the perturbation arises from a $K_{a}$ sublevel located at higher energies, as evident in Figure 4 where part of the $v_{4}$ and $v_{8}$ reduced energy level diagram is shown. Therefore the observed irregularities were attributed to second-order $a$-Coriolis interaction with $\mathrm{K}_{\mathrm{a}}=4$ of $v_{4}$. Differently, the local crossing identified in the even component of $K_{a}=5$ at $J=20 / 21$ is due to second-order $b$-Coriolis mixing with the $\mathrm{K}_{\mathrm{a}}=2$ even component of $v_{4}$. The effect of this perturbation was already observed in the $v_{4}$ spectral region [7].

The assignment of the subbands with $\mathrm{K}_{\mathrm{a}}^{\prime}>9$ was performed with care since the involved levels are influenced by stronger interactions. Actually, systematic negative residuals were observed in the transitions with $\mathrm{K}_{\mathrm{a}}{ }^{\prime} \leq 11$ and a third crossing, identified for $\mathrm{K}_{\mathrm{a}}=12$ at $\mathrm{J}=$ $14 / 15$, was ascribed to first-order $b$-Coriolis coupling with $K_{a}=11$ of $v_{4}$.

The analysis was carried out between $1180-1410 \mathrm{~cm}^{-1}$. The assignment, also including the weaker ${ }^{\mathrm{P}} \mathrm{R}$ and ${ }^{\mathrm{R}} \mathrm{P}$ series, was made up to $\mathrm{K}_{\mathrm{a}}^{\prime}=12$ with a maximum of $\mathrm{J}^{\prime}=36$ for the strongest branches.

\section{Simultaneous fit of $v_{8}$ with the $v_{3}, v_{4}, v_{5}, v_{6}$ and $v_{9}$ levels.}

The $v_{8}$ level was initially analyzed by the Watson $A$-reduced Hamiltonian in the $\mathrm{I}^{\mathrm{r}}$ representation [12]. In the preliminary fits, carried out with the ground state constants fixed at the values of [7], the upper state constants showed unsatisfactory values. This is because of interaction with the near vibrational levels. Five fundamentals, besides $v_{8}$, are located in the 
spectral region of $\mathrm{CH}_{2}$ DF below $1500 \mathrm{~cm}^{-1}: v_{3}\left(1465 \mathrm{~cm}^{-1}, \mathrm{~A}^{\prime}\right), v_{4}\left(1364 \mathrm{~cm}^{-1}, \mathrm{~A}^{\prime}\right), v_{5}(1055$ $\left.\mathrm{cm}^{-1}, \mathrm{~A}^{\prime}\right), v_{6}\left(938 \mathrm{~cm}^{-1}, \mathrm{~A}^{\prime}\right)$ and $v_{9}\left(1133 \mathrm{~cm}^{-1}, \mathrm{~A}^{\prime \prime}\right)$. From symmetry considerations it follows that $a$ - and $b$-type Coriolis resonances are allowed between vibrations of different symmetry species, whereas $c$-type Coriolis coupling and/or vibrational resonance link those of identical species. Excepting $v_{8}$, the other bands have already been studied $[5,7,8]$.

As mentioned, local crossings showing the typical Coriolis J-dependence were observed in the $v_{8}$ band and ascribed to coupling with $v_{4}$. Thus, as starting point, the low $K_{a}$ transitions of $v_{8}$ were simultaneously fitted with the data of the $v_{3} / v_{4}$ dyad, by also including a number of perturbed transitions of $v_{4}$ omitted in the earlier study. At this stage of the analysis, the second order $a$ - and $b$-type Coriolis coefficients linking $v_{4}$ and $v_{8}$ were employed. In the calculations, carried out using the SPFIT program written by Pickett [13], an uncertainty of $0.0004 \mathrm{~cm}^{-1}$ was given to lines assigned to single transitions, while for blended or very weak features the uncertainty was $0.0010 \mathrm{~cm}^{-1}$. Next, to perform refinements with higher $\mathrm{J}$ and $\mathrm{K}_{\mathrm{a}}$ transitions, first-order $a$ - and $b$-type Coriolis terms connecting $v_{8}$ with $v_{4}$ were also employed. As expected, their introduction in the fit gave an improvement and most of the spectroscopic parameters of $v_{8}$ displayed suitable values. Only the rotational constant $\mathrm{C}$ and the centrifugal distortion parameter $\Delta_{\mathrm{JK}}$ revealed rather large shifts $\left(0.003\right.$ and $\left.-0.069 \times 10^{-4} \mathrm{~cm}^{-1}\right)$ from their ground state values. These shifts compared with the corresponding ones obtained for $v_{9}$ [8], of opposite sign $\left(-0.005\right.$ and $\left.0.056 \times 10^{-4} \mathrm{~cm}^{-1}\right)$, suggested the presence of a global $c$-type Coriolis resonance. All the assigned transitions of the $v_{5}, v_{6}$ and $v_{9}$ states, mutually interacting as described in ref. [8], were then included in the data set. The preliminary values for the firstorder Coriolis couplings, linking $v_{8}$ to the perturbers, were derived from the dimensionless constants of the harmonic force field [14]. Many attempts were performed in this fitting process because the convergence was somewhat difficult to reach. A goal was that the refined upper state parameters should not significantly differ from the ground state values. However, anomalous residuals remained for transitions to $\mathrm{K}_{\mathrm{a}}=12$, especially in the crossing region with $v_{4}$, and were attributed to mutual interactions also involving $v_{5}$ and $v_{9}$. Actually, the reduced energy level scheme indicates that $K_{a}=12$ of $v_{8}$ crosses $K_{a}=11$ of $v_{4}$ and comes close to $K_{a}=$ 15 and $\mathrm{K}_{\mathrm{a}}=14$ of $v_{5}$ and $v_{9}$, respectively. The lack of experimental data concerning these levels thwarted any attempt to fit the restricted number of transitions of $v_{8}$ with $\mathrm{K}_{\mathrm{a}}^{\prime}=12$ and so they were omitted from the final refinement. It should be recalled here that an extension of 
the analysis is mainly dependent on new measurements of the $v_{9}$ band which is very weak and only observed in the spectrum through perturbation-allowed transitions by intensity borrowing from the very strong $v_{5}$ band [8].

The best representation of the interacting level system was finally given by a model involving seven first- and three second-order Coriolis terms together with two second-order vibrational resonance constants, as illustrated in Figure 5. The interaction matrix elements used in the model are summarized, for convenience, in Table 1. The spectroscopic parameters obtained from a fit comprising most of the assigned transitions are given in Tables 2 and 3. The results have been obtained with a standard deviation of $0.107 \times 10^{-2} \mathrm{~cm}^{-1}$ which is higher than the accuracy of the fitted lines but comparable to that obtained in the previous analysis $[7,8]$. Table 2 collects the upper state parameters of the $v_{3} / v_{4} / v_{5} / v_{6} / v_{8} / v_{9}$ hexade and, for completeness, the ground state constants. On the whole the refined parameters appear well determined. In particular the $\mathrm{C}$ and $\Delta_{J K}$ terms of $v_{9}$, that in the previous analysis were deviating from the ground state values, are now more suitable. Where consistent with the available experimental data, the parameters $\delta_{\mathrm{J}}$ and $\delta_{\mathrm{k}}$ were refined, otherwise they were constrained to the ground state values. No sextic centrifugal distortion constants could be obtained with significance. However, it should be mentioned that some resonance contributions, which the model is unable to account for, still remain.

The Coriolis and Fermi term values are listed in Table 3. To maintain the flexibility of the model, notwithstanding the existence of strong correlations with the molecular parameters, all the coupling coefficients relevant for the analysis were refined. The table also provides, in square brackets, the computed values of the seven first-order Coriolis parameters, derived from the dimensionless constants of the harmonic force field of methyl fluoride [14]. The comparison with the empirical values show generally a satisfactory agreement. The larger difference concerns the smaller coupling coefficient $\xi_{4,8}^{b}$, probably not too well determined in the refinement.

To test the reliability of the obtained parameters, simulations were performed in different spectral regions of $v_{8}$. A small portion of the synthetic spectrum is reported in the upper trace of Figure 3. It matches very well with the experimental one (lower trace) and reproduces the anomalous splittings confirming that the employed model satisfactorily represents the system involving the $v_{3}, v_{4}, v_{5}, v_{6}, v_{8}$, and $v_{9}$ interacting levels. 
In conclusion, the high-resolution infrared study of the strongly perturbed $v_{8}$ level of $\mathrm{CH}_{2} \mathrm{DF}$ has been reported for the first time. The spectrum has been analyzed in detail and the observed irregularities were attributed to different Coriolis resonances with $v_{4}$ and $v_{9}$, in turn coupled with other levels. For this reason the previously analyzed systems were included in the data set of $v_{8}$ and, from a combined analysis of six interacting states, a set of accurate spectroscopic constants, which satisfactory reproduces the experimental features within the measurement accuracy, was determined. With this work all levels of $\mathrm{CH}_{2} \mathrm{DF}$ located below $1500 \mathrm{~cm}^{-1}$ have been characterized, providing a large amount of data which should give valuable information to test the existing anharmonic potential energy surface and to determine an improved cubic force field of methyl fluoride.

A complete list of the transitions used in the final fit has been deposited as Supplementary online material.

\section{Acknowledgements}

This work has been supported by "PRIN 2007" funds (project "Trasferimenti di energia, carica e molecole in sistemi complessi") and by University of Venice (FRA funds). The authors gratefully acknowledge A. Baldan for the synthesis of the sample. 


\section{References}

[1] J. Demaison, J. Breidung, W. Thiel, D. Papoušek, Struct. Chem., 10, 129 (1999).

[2] S. Kondo, Y. Koga, T. Nakanaga, J. Chem. Phys., 81, 1951 (1984).

[3] I. A. Atkinson, M. M. Law, Spectrochim. Acta, 58, 873 (2002).

[4] J. R. Riter, D. F. Eggers, J. Chem. Phys., 44, 745 (1966).

[5] I. A. Atkinson, M. M. Law, J. Molec. Spectrosc., 206, 135 (2001).

[6] W. W. Clark, F. C. De Lucia, J. Mol. Struct., 32, 29 (1976).

[7] A. Baldacci, R. Visinoni, G. Nivellini, Mol. Phys., 100, 22 (2002).

[8] A. Baldacci, R. Visinoni, G. Nivellini, Mol. Phys., 102, 1731 (2004).

[9] W. F. Edgell, L. Parts, J. Amer. Soc., 77, 4899 (1955).

[10] D. Papousek, J. F. Olgivie, S. Civiš, M. Winnewisser, J. Molec. Spectrosc., 149, 109 (1991).

[11] T. Nakagawa, J. Overend, J. Mol. Spectrosc., 50, 333 (1974).

[12] J. K. Watson, "Vibrational Spectroscopy and Structure" (Elsevier, Amsterdam, 1977), Vol. 6, pp 1-89.

[13] H. M. Pickett, J. Mol. Spectrosc., 148, 371 (1991).

[14] M. M. Law, J. L. Duncan, I. M. Mills, J. Mol. Struct., 92, 323 (1992). 


\section{Figure captions}

Figure 1. Overview of the low resolution $\left(0.5 \mathrm{~cm}^{-1}\right)$ IR spectrum of the $v_{8} c$-type band of $\mathrm{CH}_{2} \mathrm{DF}(\mathrm{P}=24 \mathrm{kPa}$, path length $10 \mathrm{~cm})$. The R-branch merges with the weaker hybrid $v_{4}$ band. The high resolution spectrum of the central peak marked with $*$ is reproduced in Figure 2.

Figure 2. Spectral portion of the ${ }^{\mathrm{R}} \mathrm{Q}_{0}(\mathrm{~J})$ multiplet of the $v_{8}$ band of $\mathrm{CH}_{2} \mathrm{DF}(\mathrm{P}=67 \mathrm{~Pa}$, path length $5 \mathrm{~m}$ ) showing a turnover in the assignment at $\mathrm{J}=21$. Many weaker unlabelled lines belong to different ${ }^{\mathrm{R}} \mathrm{P}$ and ${ }^{\mathrm{P}} \mathrm{R}$ subbranches with low $\mathrm{K}_{\mathrm{a}}$ values.

Figure 3. The ${ }^{\mathrm{p}} \mathrm{Q}_{7}(\mathrm{~J})$ multiplet of the $v_{8}$ band of $\mathrm{CH}_{2} \mathrm{DF}$ (lower trace; $\mathrm{P}=67 \mathrm{~Pa}$, path length 5 $\mathrm{m}$ ) is compared with the simulated spectrum (upper trace). The local resonance observed at $J=24 / 25$ and $J=25 / 26$, attributed to crossings with $K_{a}=4$ of $v_{4}$, produces split lines with intensity depletion compared to the regular series of the $\mathbf{J}$ manifold.

Figure 4. Part of the reduced energy level diagram of $v_{4}$ and $v_{8}$ of $\mathrm{CH}_{2} \mathrm{DF}$. The energies have been calculated from the upper state constants of Table 3 . The $v_{4}$ sublevels are indicated by dashed lines, those of $v_{8}$ by full lines. The $\Delta \mathrm{K}_{\mathrm{a}}= \pm 2$ (square) and $\Delta \mathrm{K}_{\mathrm{a}}= \pm 3$ (circle) crossings are due to $a$ - and $b$-type Coriolis resonances.

Figure 5. Vibrational energy levels of $\mathrm{CH}_{2} \mathrm{DF}$ below $1500 \mathrm{~cm}^{-1}$. The figure shows the level symmetry and the interaction parameters employed to analyze the $v_{3} / v_{4} / v_{5} / v_{6} /$ $v_{8} / v_{9}$ hexade. $\xi_{\mathrm{r}, \mathrm{s}}^{\mathrm{a}}(\mathrm{a}=a, b, c)$ denotes first-order Coriolis interaction between $\mathrm{v}_{\mathrm{r}}$ and $\mathrm{v}_{\mathrm{s}} ; \eta_{\mathrm{r}, \mathrm{s}}^{b, c}$ and $\theta_{\mathrm{r}, \mathrm{s}}^{b}$ indicate second order $a$ - and $b$-Coriolis coupling while $W_{\mathrm{r}, \mathrm{s}}$ refers to second order Fermi resonance. 


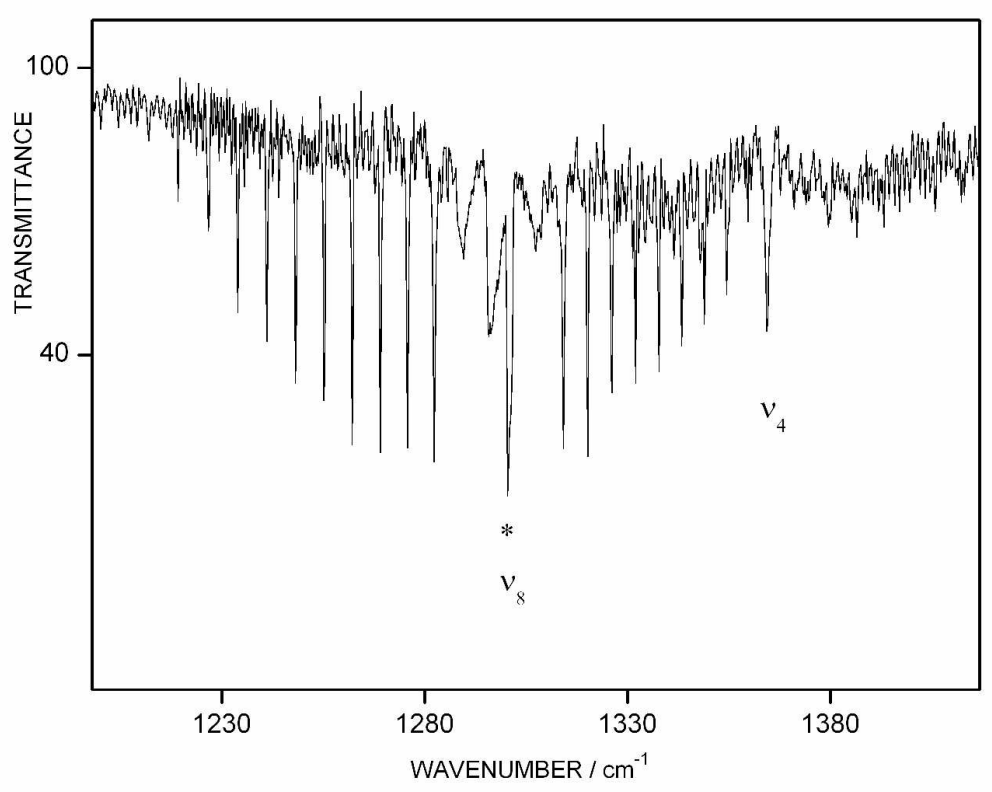

$283 \times 203 \mathrm{~mm}(300 \times 300$ DPI $)$ 


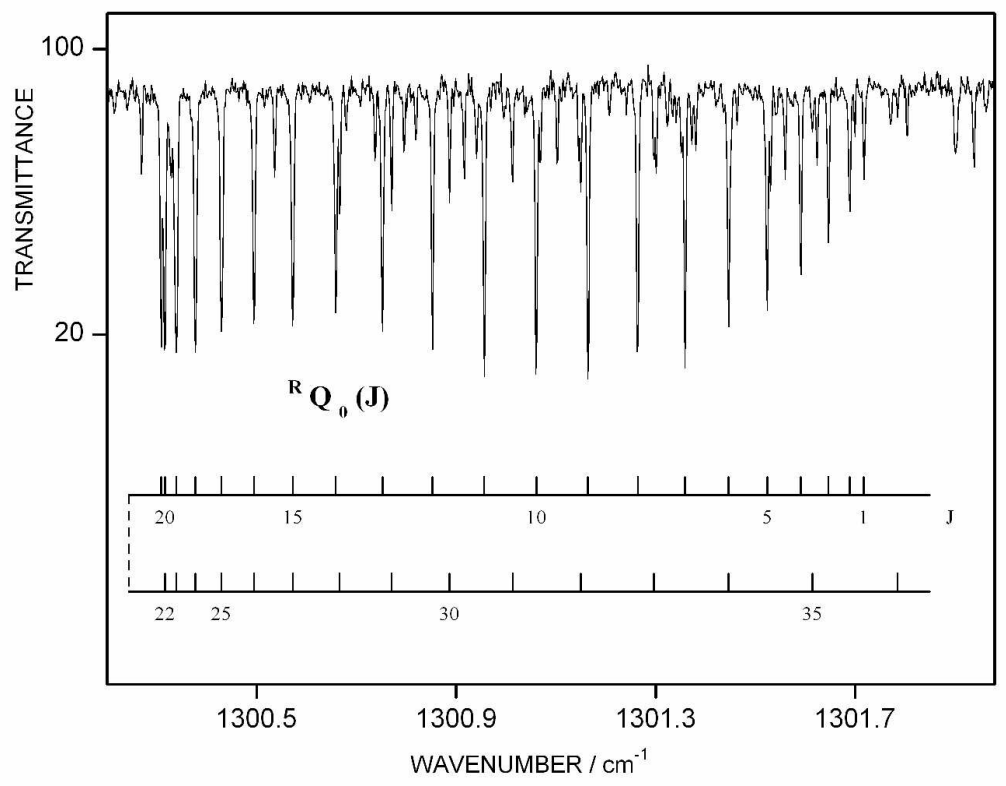

$283 \times 203 \mathrm{~mm}(300 \times 300$ DPI $)$

URL: http://mc.manuscriptcentral.com/tandf/tmph 


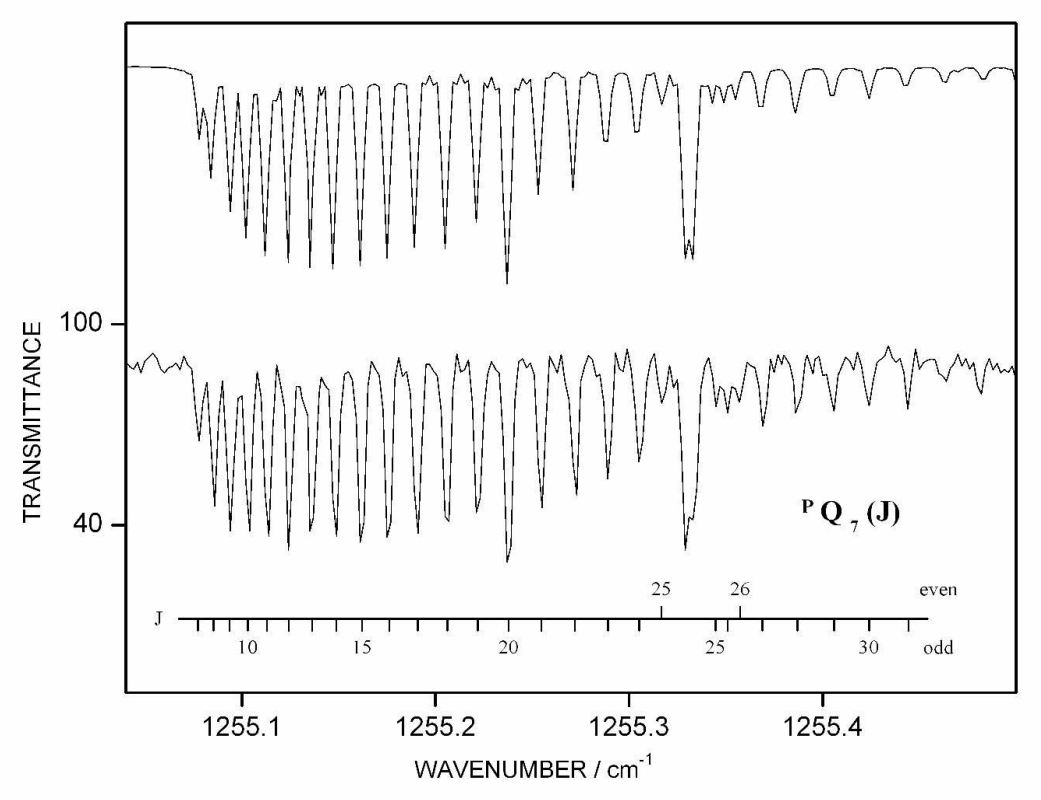

$283 \times 203 \mathrm{~mm}(300 \times 300 \mathrm{DPI})$

URL: http://mc.manuscriptcentral.com/tandf/tmph 


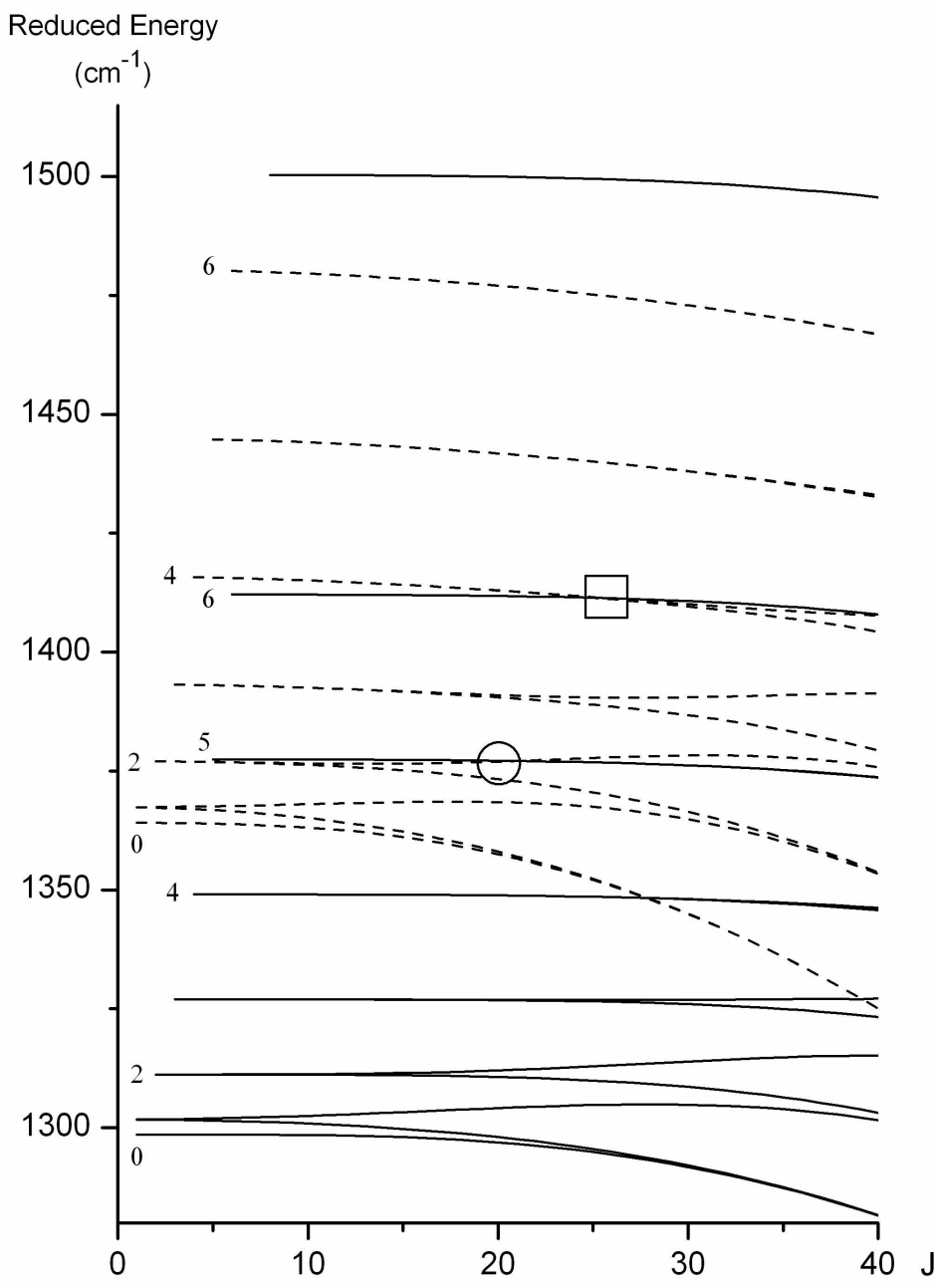

$203 \times 283 \mathrm{~mm}(300 \times 300$ DPI $)$

URL: http://mc.manuscriptcentral.com/tandf/tmph 
1

2

3

4

5

6

7

8

9

10

11

12

13

14

15

16

17

18

19

20

21

22

23

24

25

26

27

28

29

30

31

32

33

34

35

36

37

38

39

40

41

42

43

44

45

46

47

48

49

50

51

52

53

54

55

56

57

58

59

60

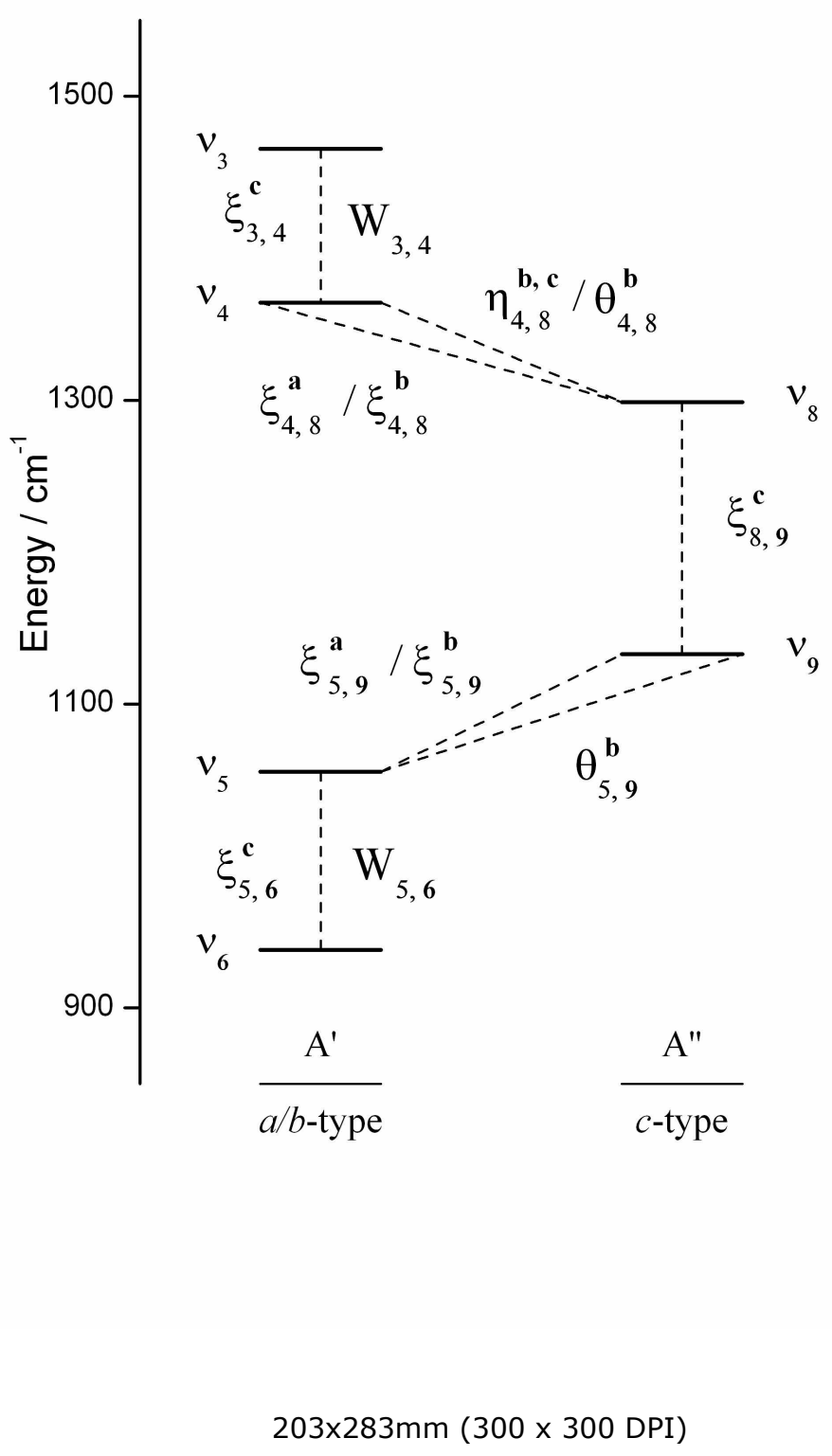

URL: http://mc.manuscriptcentral.com/tandf/tmph 
Table 1. Interaction matrix elements of the Hamiltonian in symmetric top basis

Type Resonance Interaction Matrix Elements

First order $a$-Coriolis $\quad<\mathrm{v}_{\mathrm{r}}=1, \mathrm{~J}, \mathrm{~K}|\mathbf{H}| \mathrm{v}_{\mathrm{s}}=1, \mathrm{~J}, \mathrm{~K}>=\xi_{\mathrm{r}, \mathrm{s}}^{a} \cdot \mathrm{K}$

First order $b$-Coriolis $\quad<\mathrm{v}_{\mathrm{r}}=1, \mathrm{~J}, \mathrm{~K}|\mathbf{H}| \mathrm{v}_{\mathrm{s}}=1, \mathrm{~J}, \mathrm{~K} \pm 1>=\frac{1}{2} \cdot \xi_{\mathrm{r}, \mathrm{s}}^{b} \cdot \mathrm{F}(\mathrm{J}, \mathrm{K})$

First order $c$-Coriolis $\quad<\mathrm{v}_{\mathrm{r}}=1, \mathrm{~J}, \mathrm{~K}|\mathrm{H}| \mathrm{v}_{\mathrm{s}}=1, \mathrm{~J}, \mathrm{~K} \pm 1>= \pm \frac{1}{2} \cdot \xi_{\mathrm{r}, \mathrm{s}}^{c} \cdot \mathrm{F}(\mathrm{J}, \mathrm{K})$

Second order $a$-Coriolis $\quad<\mathrm{v}_{\mathrm{r}}=1, \mathrm{~J}, \mathrm{~K}|\mathbf{H}| \mathrm{v}_{\mathrm{s}}=1, \mathrm{~J}, \mathrm{~K} \pm 2>=\mp \frac{1}{2} \cdot \eta_{\mathrm{r}, \mathrm{s}}^{b c} \cdot \mathrm{F}(\mathrm{J}, \mathrm{K}) \cdot \mathrm{F}(\mathrm{J}, \mathrm{K} \pm 1)$

Second order $b$-Coriolis $\quad<\mathrm{v}_{\mathrm{r}}=1, \mathrm{~J}, \mathrm{~K}|\mathbf{H}| \mathrm{v}_{\mathrm{s}}=1, \mathrm{~J}, \mathrm{~K} \pm 3>= \pm \frac{1}{8} \cdot \Theta_{\mathrm{r}, \mathrm{s}}^{b} \cdot \mathrm{F}(\mathrm{J}, \mathrm{K}) \cdot \mathrm{F}(\mathrm{J}, \mathrm{K} \pm 1) \cdot \mathrm{F}(\mathrm{J}, \mathrm{K} \pm 2)$

Second order Fermi $\quad<\mathrm{v}_{\mathrm{r}}=1, \mathrm{~J}, \mathrm{~K}|\mathbf{H}| \mathrm{v}_{\mathrm{s}}=1, \mathrm{~J}, \mathrm{~K} \pm 2>=\frac{1}{2} \cdot \mathrm{W}_{\mathrm{r}, \mathrm{s}} \cdot \mathrm{F}(\mathrm{J}, \mathrm{K}) \cdot \mathrm{F}(\mathrm{J}, \mathrm{K} \pm 1)$

$$
\begin{aligned}
& \mathrm{F}(\mathrm{J}, \mathrm{K})=[\mathrm{J}(\mathrm{J}+1)-\mathrm{K}(\mathrm{K} \pm 1)]^{1 / 2} \\
& \xi_{\mathrm{r}, \mathrm{s}}^{a}=\mathrm{A}_{e} \cdot \zeta_{\mathrm{r}, \mathrm{s}}^{a} \cdot\left(\omega_{\mathrm{r}}+\omega_{\mathrm{s}}\right) /\left(\omega_{\mathrm{r}} \cdot \omega_{\mathrm{s}}\right)^{1 / 2} \\
& \xi_{\mathrm{r}, \mathrm{s}}^{b}=\mathrm{B}_{e} \cdot \zeta_{\mathrm{r}, \mathrm{s}}^{b} \cdot\left(\omega_{\mathrm{r}}+\omega_{\mathrm{s}}\right) /\left(\omega_{\mathrm{r}} \cdot \omega_{\mathrm{s}}\right)^{1 / 2} \\
& \xi_{\mathrm{r}, \mathrm{s}}^{c}=\mathrm{C}_{e} \cdot \zeta_{\mathrm{r}, \mathrm{s}}^{c} \cdot\left(\omega_{\mathrm{r}}+\omega_{\mathrm{s}}\right) /\left(\omega_{\mathrm{r}} \cdot \omega_{\mathrm{s}}\right)^{1 / 2}
\end{aligned}
$$


Table 2. Spectroscopic parameters $\left(\mathrm{cm}^{-1}\right)$ for the $v_{3}, v_{4}, v_{5}, v_{6}, v_{8}, v_{9}$ band system of $\mathrm{CH}_{2} \mathrm{DF}^{\mathrm{a}}$

\begin{tabular}{|c|c|c|c|c|c|c|c|}
\hline & Ground State $^{\mathrm{b}}$ & $v_{3}$ & $v_{4}$ & $v_{5}$ & $v_{6}$ & $v_{8}$ & $v_{9}$ \\
\hline$v_{0}$ & & $1465.477574(26)$ & $1364.168036(32)$ & $1055.544760(37)$ & $938.300406(50)$ & $1298.575644(29)$ & $1132.78084(27)$ \\
\hline$A$ & 3.99193092 & $3.9931639(17)$ & $4.001852(97)$ & $3.984741(21)$ & $3.9924941(24)$ & $3.948220(97)$ & $4.002762(23)$ \\
\hline$B$ & 0.80200182 & $0.80376852(34)$ & $0.8024725(20)$ & $0.795222(14)$ & $0.79885475(44)$ & $0.7993000(21)$ & $0.797977(15)$ \\
\hline$C$ & 0.76584315 & $0.7637980(47)$ & $0.7664034(49)$ & $0.7564945(32)$ & $0.7640131(33)$ & $0.765618(18)$ & $0.764134(17)$ \\
\hline$\Delta_{J} \times 10^{5}$ & 0.164497 & $0.177118(17)$ & $0.157725(40)$ & $0.172092(38)$ & $0.157312(56)$ & $0.166028(17)$ & $0.16673(16)$ \\
\hline$\Delta_{J K} \times 10^{4}$ & 0.114538 & $0.127688(99)$ & $0.095506(77)$ & $0.108259(69)$ & $0.116286(74)$ & $0.09643(22)$ & $0.13727(28)$ \\
\hline$\Delta_{K} \times 10^{4}$ & 0.45993 & $0.47221(12)$ & $0.49182(78)$ & $0.44923(15)$ & $0.47274(24)$ & $0.40995(79)$ & $0.49874(84)$ \\
\hline$\delta_{J} \times 10^{7}$ & 0.77177 & $0.5643(15)$ & $1.0934(42)$ & $0.4089(64)$ & $0.77177^{c}$ & $0.6198(16)$ & $0.77177^{c}$ \\
\hline$\delta_{K} \times 10^{5}$ & 0.1826 & $1.4445(55)$ & $0.5656(55)$ & $0.22981(99)$ & $0.1826^{\mathrm{c}}$ & $0.16182(99)$ & $0.1826^{\mathrm{c}}$ \\
\hline \multicolumn{8}{|c|}{${ }^{\mathrm{d}} \Phi_{\mathrm{JK}} \times 10^{10}=0.587, \Phi_{\mathrm{KJ}} \times 10^{9}=0.203, \Phi_{\mathrm{K}} \times 10^{8}=0.237$} \\
\hline$\sigma \times 10^{3}$ & & 1.072 & & & & & \\
\hline No. of Data & & 2029 & 1275 & 1007 & 588 & 1737 & 210 \\
\hline $\mathbf{J}_{\max }$ & & 37 & 28 & 33 & 27 & 36 & 26 \\
\hline $\mathrm{K}_{\mathrm{a} \max }$ & & 11 & 10 & 12 & 10 & 11 & 10 \\
\hline
\end{tabular}

${ }^{\mathrm{a}}$ Quoted uncertainties are one standard deviation in units of the last significant digit.

${ }^{\mathrm{b}}$ From [7].

${ }^{\mathrm{c}}$ Fixed at the ground state value.

${ }^{\mathrm{d}}$ Ground state sextic coefficients from [7] fixed for the upper levels.

URL: http://mc.manuscriptcentral.com/tandf/tmph 
Table 3. Coriolis and Fermi coupling values $\left(\mathrm{cm}^{-1}\right)$ obtained from the fit of the $v_{3}, v_{4}, v_{5}, v_{6}, v_{8}, v_{9}$ band system of $\mathrm{CH}_{2} \mathrm{DF}^{\mathrm{a}}$

\begin{tabular}{|c|c|c|c|c|c|}
\hline Type Resonance & \multicolumn{3}{|c|}{ First Order ${ }^{b}$} & \multicolumn{2}{|r|}{ Second Order } \\
\hline$a$-type Coriolis & $\xi_{4,8}^{a}$ & $0.7433(43)$ & {$[0.9607]$} & $\eta_{4,8}^{b c}$ & $0.8629(56) \times 10^{-3}$ \\
\hline \multirow{3}{*}{$b$-type Coriolis } & $\xi_{5,9}^{a}$ & $0.98720(81)$ & {$[1.0687]$} & & \\
\hline & $\xi_{4,8}^{b}$ & $-0.19524(35)$ & {$[0.1386]$} & $\Theta_{4,8}^{b}$ & $0.11100(19) \times 10^{-1}$ \\
\hline & $\xi_{5,9}^{b}$ & $0.72490(76)$ & {$[0.6968]$} & $\Theta_{5,9}^{b}$ & $-0.3126(30) \times 10^{-2}$ \\
\hline \multirow[t]{3}{*}{$c$-type Coriolis } & $\xi_{3,4}^{c}$ & $1.25572(19)$ & [1.1787] & & \\
\hline & $\xi_{5,6}^{c}$ & $0.72747(26)$ & {$[0.5653]$} & & \\
\hline & $\xi_{8,9}^{c}$ & $0.7882(19)$ & [0.8639] & & \\
\hline \multirow[t]{2}{*}{ Fermi } & & & & $\mathrm{W}_{3,4}$ & $-0.856768(33) \times 10^{-3}$ \\
\hline & & & & $\mathrm{W}_{5,6}$ & $0.174779(33) \times 10^{-3}$ \\
\hline
\end{tabular}

\footnotetext{
${ }^{a}$ Quoted uncertainties are one standard deviation in units of the last significant digit.

$\mathrm{b}$ The parameters in brackets were derived from the harmonic force field [14].
} 
High resolution IR spectrum of the $v_{8}$ band of $\mathrm{CH}_{2} \mathrm{DF}$ : analysis of the interactions with the near vibrational levels

\author{
R. Visinoni, A. Baldacci \\ Università $\mathrm{Ca}^{\prime}$ Foscari di Venezia \\ Dipartimento di Chimica Fisica \\ D. D. 2137, I-30123 Venezia, Italy
}

and

G. Nivellini

Università di Bologna

Dipartimento di Chimica Fisica e Inorganica

Viale del Risorgimento 4, I-40136 Bologna, Italy

List of the fitted transitions of $v_{3}$ (p. 2-15), $v_{4}$ (p. 15-23), $v_{5}$ (p. 23-30), $v_{6}$ (p. 30-33), $v_{8}$ (p. 33-45) and $v_{9}$ (p. 45-46) bands.

Each column reports:

$\mathrm{J}^{\prime}, \mathrm{K}_{\mathrm{a}}{ }^{\prime}, \mathrm{K}_{\mathrm{c}}{ }^{\prime}, \mathrm{J}^{\prime}, \mathrm{K}_{\mathrm{a}}{ }^{\prime}, \mathrm{K}_{\mathrm{c}}{ }^{\prime}$, Obs $\left(\mathrm{cm}^{-1}\right)$, (Obs. - Calc. ) x $10^{4}\left(\mathrm{~cm}^{-1}\right)$, Uncertainty x $10^{4}\left(\mathrm{~cm}^{-1}\right)$. 


\begin{tabular}{|c|c|c|c|c|c|c|c|}
\hline 19 & 11 & 8 & 20 & 12 & 9 & 1364.7098 & 13 \\
\hline 18 & 11 & 7 & 19 & 12 & 8 & 1365.7453 & 15 \\
\hline 17 & 11 & 6 & 18 & 12 & 7 & 1366.7928 & 18 \\
\hline 22 & 10 & 12 & 23 & 11 & 13 & 1367.5174 & 1 \\
\hline 16 & 11 & 5 & 17 & 12 & 6 & 1367.8524 & 10 \\
\hline 21 & 10 & 11 & 22 & 11 & 12 & 1368.5839 & 0 \\
\hline 15 & 11 & 4 & 16 & 12 & 5 & 1368.9274 & 13 \\
\hline 20 & 10 & 10 & 21 & 11 & 11 & 1369.6620 & 1 \\
\hline 14 & 11 & 3 & 15 & 12 & 4 & 1370.0171 & 4 \\
\hline 19 & 10 & 9 & 20 & 11 & 10 & 1370.7522 & 1 \\
\hline 13 & 11 & 2 & 14 & 12 & 3 & 1371.1249 & 2 \\
\hline 12 & 11 & 1 & 13 & 12 & 2 & 1372.2508 & -11 \\
\hline 17 & 10 & 7 & 18 & 11 & 8 & 1372.9736 & 8 \\
\hline 11 & 11 & 0 & 12 & 12 & 1 & 1373.3980 & -21 \\
\hline 16 & 10 & 6 & 17 & 11 & 7 & 1374.1061 & 7 \\
\hline 21 & 9 & 12 & 22 & 10 & 13 & 1374.6261 & 2 \\
\hline 15 & 10 & 5 & 16 & 11 & 6 & 1375.2550 & 8 \\
\hline 20 & 9 & 11 & 21 & 10 & 12 & 1375.7529 & 9 \\
\hline 14 & 10 & 4 & 15 & 11 & 5 & 1376.4210 & 7 \\
\hline 19 & 9 & 10 & 20 & 10 & 11 & 1376.8921 & 11 \\
\hline 13 & 10 & 3 & 14 & 11 & 4 & 1377.6048 & -2 \\
\hline 18 & 9 & 9 & 19 & 10 & 10 & 1378.0459 & 21 \\
\hline 12 & 10 & 2 & 13 & 11 & 3 & 1378.8088 & -6 \\
\hline 17 & 9 & 8 & 18 & 10 & 9 & 1379.2116 & 4 \\
\hline 22 & 8 & 14 & 23 & 9 & 15 & 1379.5938 & 9 \\
\hline 11 & 10 & 1 & 12 & 11 & 2 & 1380.0333 & -15 \\
\hline 16 & 9 & 7 & 17 & 10 & 8 & 1380.3950 & 10 \\
\hline 21 & 8 & 13 & 22 & 9 & 14 & 1380.7480 & 7 \\
\hline 10 & 10 & 0 & 11 & 11 & 1 & 1381.2803 & -22 \\
\hline 15 & 9 & 6 & 16 & 10 & 7 & 1381.5938 & 8 \\
\hline 20 & 8 & 12 & 21 & 9 & 13 & 1381.9137 & 9 \\
\hline 25 & 7 & 18 & 26 & 8 & 19 & 1382.3596 & 7 \\
\hline 14 & 9 & 5 & 15 & 10 & 6 & 1382.8094 & 2 \\
\hline 19 & 8 & 11 & 20 & 9 & 12 & 1383.0907 & 1 \\
\hline 24 & 7 & 17 & 25 & 8 & 18 & 1383.4981 & -1 \\
\hline 13 & 9 & 4 & 14 & 10 & 5 & 1384.0438 & 3 \\
\hline 18 & 8 & 10 & 19 & 9 & 11 & 1384.2818 & 4 \\
\hline 23 & 7 & 16 & 24 & 8 & 17 & 1384.6498 & 11 \\
\hline 12 & 9 & 3 & 13 & 10 & 4 & 1385.2965 & -1 \\
\hline 17 & 8 & 9 & 18 & 9 & 10 & 1385.4871 & 7 \\
\hline 22 & 7 & 15 & 23 & 8 & 16 & 1385.8126 & 15 \\
\hline 11 & 9 & 2 & 12 & 10 & 3 & 1386.5687 & -9 \\
\hline 16 & 8 & 8 & 17 & 9 & 9 & 1386.7077 & 15 \\
\hline 21 & 7 & 14 & 22 & 8 & 15 & 1386.9865 & 7 \\
\hline 10 & 9 & 1 & 11 & 10 & 2 & 1387.8621 & -11 \\
\hline 15 & 8 & 7 & 16 & 9 & 8 & 1387.9421 & 5 \\
\hline 20 & 7 & 13 & 21 & 8 & 14 & 1388.1737 & 2 \\
\hline 25 & 6 & 19 & 26 & 7 & 20 & 1388.4869 & -6 \\
\hline 9 & 9 & 0 & 10 & 10 & 1 & 1389.1767 & -17 \\
\hline 14 & 8 & 6 & 15 & 9 & 7 & 1389.1937 & 3 \\
\hline 19 & 7 & 12 & 20 & 8 & 13 & 1389.3758 & 11 \\
\hline 24 & 6 & 18 & 25 & 7 & 19 & 1389.6504 & 2 \\
\hline 13 & 8 & 5 & 14 & 9 & 6 & 1390.4623 & 0 \\
\hline 18 & 7 & 11 & 19 & 8 & 12 & 1390.5905 & 4 \\
\hline 23 & 6 & 17 & 24 & 7 & 18 & 1390.8246 & 6 \\
\hline 12 & 8 & 4 & 13 & 9 & 5 & 1391.7484 & -6 \\
\hline 17 & 7 & 10 & 18 & 8 & 11 & 1391.8208 & 3 \\
\hline 22 & 6 & 16 & 23 & 7 & 17 & 1392.0106 & 11 \\
\hline 27 & 5 & 22 & 28 & 6 & 23 & 1392.3440 & -5 \\
\hline 13 & 11 & 2 & 13 & 12 & 1 & 1393.0121 & -1 \\
\hline 11 & 8 & 3 & 12 & 9 & 4 & 1393.0537 & -5 \\
\hline 16 & 7 & 9 & 17 & 8 & 10 & 1393.0671 & 1 \\
\hline 21 & 6 & 15 & 22 & 7 & 16 & 1393.2077 & 6 \\
\hline 14 & 11 & 3 & 14 & 12 & 2 & 1393.4645 & -3 \\
\hline 26 & 5 & 21 & 27 & 6 & 22 & 1393.5090 & 10 \\
\hline 15 & 7 & 8 & 16 & 8 & 9 & 1394.3318 & -2 \\
\hline 10 & 8 & 2 & 11 & 9 & 3 & 1394.3778 & -8 \\
\hline 16 & 11 & 5 & 16 & 12 & 4 & 1394.4185 & -7 \\
\hline 20 & 6 & 14 & 21 & 7 & 15 & 1394.4185 & 12 \\
\hline 25 & 5 & 20 & 26 & 6 & 21 & 1394.6823 & 13 \\
\hline 17 & 11 & 6 & 17 & 12 & 5 & 1394.9194 & 16 \\
\hline 18 & 11 & 7 & 18 & 12 & 6 & 1395.4315 & 27 \\
\hline
\end{tabular}




\begin{tabular}{|c|c|c|c|c|c|c|c|}
\hline 8 & 6 & 2 & 9 & 7 & 3 & 1410.0909 & -5 \\
\hline 17 & 4 & 13 & 18 & 5 & 14 & 1410.8101 & 8 \\
\hline 25 & 9 & 16 & 25 & 10 & 15 & 1410.8332 & -18 \\
\hline 22 & 3 & 20 & 23 & 4 & 19 & 1410.8401 & 23 \\
\hline 22 & 3 & 19 & 23 & 4 & 20 & 1410.9427 & -5 \\
\hline 12 & 5 & 7 & 13 & 6 & 8 & 1411.0278 & -1 \\
\hline 26 & 9 & 17 & 26 & 10 & 16 & 1411.3117 & -32 \\
\hline 9 & 8 & 1 & 9 & 9 & 0 & 1411.3753 & -19 \\
\hline 7 & 6 & 1 & 8 & 7 & 2 & 1411.5061 & -3 \\
\hline 10 & 8 & 2 & 10 & 9 & 1 & 1411.5969 & -2 \\
\hline 27 & 9 & 18 & 27 & 10 & 17 & 1411.7978 & -45 \\
\hline 11 & 8 & 3 & 11 & 9 & 2 & 1411.8360 & -5 \\
\hline 21 & 3 & 19 & 22 & 4 & 18 & 1412.0892 & 7 \\
\hline 12 & 8 & 4 & 12 & 9 & 3 & 1412.0939 & -6 \\
\hline 16 & 4 & 12 & 17 & 5 & 13 & 1412.1161 & 2 \\
\hline 21 & 3 & 18 & 22 & 4 & 19 & 1412.1671 & -3 \\
\hline 28 & 2 & 26 & 29 & 3 & 27 & 1412.2681 & -10 \\
\hline 13 & 8 & 5 & 13 & 9 & 4 & 1412.3706 & 0 \\
\hline 11 & 5 & 6 & 12 & 6 & 7 & 1412.3854 & -1 \\
\hline 14 & 8 & 6 & 14 & 9 & 5 & 1412.6642 & 2 \\
\hline 6 & 6 & 0 & 7 & 7 & 1 & 1412.9393 & -5 \\
\hline 15 & 8 & 7 & 15 & 9 & 6 & 1412.9746 & 6 \\
\hline 27 & 2 & 25 & 28 & 3 & 26 & 1413.1736 & -11 \\
\hline 16 & 8 & 8 & 16 & 9 & 7 & 1413.3005 & 7 \\
\hline 20 & 3 & 18 & 21 & 4 & 17 & 1413.3493 & 6 \\
\hline 20 & 3 & 17 & 21 & 4 & 18 & 1413.4067 & -3 \\
\hline 15 & 4 & 11 & 16 & 5 & 12 & 1413.4287 & 2 \\
\hline 17 & 8 & 9 & 17 & 9 & 8 & 1413.6412 & 6 \\
\hline 10 & 5 & 5 & 11 & 6 & 6 & 1413.7591 & -1 \\
\hline 18 & 8 & 10 & 18 & 9 & 9 & 1413.9964 & 8 \\
\hline 19 & 8 & 11 & 19 & 9 & 10 & 1414.3649 & 9 \\
\hline 19 & 3 & 17 & 20 & 4 & 16 & 1414.6196 & 3 \\
\hline 19 & 3 & 16 & 20 & 4 & 17 & 1414.6620 & 2 \\
\hline 20 & 8 & 12 & 20 & 9 & 11 & 1414.7453 & 4 \\
\hline 14 & 4 & 10 & 15 & 5 & 11 & 1414.7528 & -1 \\
\hline 23 & 2 & 22 & 24 & 3 & 21 & 1414.9681 & -1 \\
\hline 21 & 8 & 13 & 21 & 9 & 12 & 1415.1379 & 7 \\
\hline 9 & 5 & 4 & 10 & 6 & 5 & 1415.1495 & -1 \\
\hline 22 & 8 & 14 & 22 & 9 & 13 & 1415.5387 & -11 \\
\hline 18 & 3 & 16 & 19 & 4 & 15 & 1415.9003 & -7 \\
\hline 18 & 3 & 15 & 19 & 4 & 16 & 1415.9313 & -1 \\
\hline 23 & 8 & 15 & 23 & 9 & 14 & 1415.9499 & -14 \\
\hline 13 & 4 & 9 & 14 & 5 & 10 & 1416.0905 & -5 \\
\hline 24 & 8 & 16 & 24 & 9 & 15 & 1416.3669 & -30 \\
\hline 22 & 2 & 21 & 23 & 3 & 20 & 1416.3811 & 2 \\
\hline 8 & 5 & 3 & 9 & 6 & 4 & 1416.5565 & -2 \\
\hline 25 & 8 & 17 & 25 & 9 & 16 & 1416.7871 & -53 \\
\hline 23 & 2 & 21 & 24 & 3 & 22 & 1417.1083 & -22 \\
\hline 17 & 3 & 15 & 18 & 4 & 14 & 1417.1946 & 1 \\
\hline 17 & 3 & 14 & 18 & 4 & 15 & 1417.2159 & 0 \\
\hline 12 & 4 & 8 & 13 & 5 & 9 & 1417.4433 & -2 \\
\hline 8 & 7 & 1 & 8 & 8 & 0 & 1417.6985 & -5 \\
\hline 21 & 2 & 20 & 22 & 3 & 19 & 1417.7818 & 16 \\
\hline 9 & 7 & 2 & 9 & 8 & 1 & 1417.8794 & -9 \\
\hline 7 & 5 & 2 & 8 & 6 & 3 & 1417.9808 & -2 \\
\hline 10 & 7 & 3 & 10 & 8 & 2 & 1418.0791 & -4 \\
\hline 22 & 2 & 20 & 23 & 3 & 21 & 1418.1713 & -13 \\
\hline 11 & 7 & 4 & 11 & 8 & 3 & 1418.2950 & -3 \\
\hline 16 & 3 & 14 & 17 & 4 & 13 & 1418.5002 & -1 \\
\hline 16 & 3 & 13 & 17 & 4 & 14 & 1418.5148 & -3 \\
\hline 12 & 7 & 5 & 12 & 8 & 4 & 1418.5235 & 0 \\
\hline 13 & 7 & 6 & 13 & 8 & 5 & 1418.7328 & 9 \\
\hline 11 & 4 & 7 & 12 & 5 & 8 & 1418.8111 & 0 \\
\hline 14 & 7 & 7 & 14 & 8 & 6 & 1419.1032 & -5 \\
\hline 20 & 2 & 19 & 21 & 3 & 18 & 1419.1696 & 5 \\
\hline 21 & 2 & 19 & 22 & 3 & 20 & 1419.2659 & 6 \\
\hline 15 & 7 & 8 & 15 & 8 & 7 & 1419.3711 & -2 \\
\hline 6 & 5 & 1 & 7 & 6 & 2 & 1419.4223 & -3 \\
\hline 16 & 7 & 9 & 16 & 8 & 8 & 1419.6679 & -2 \\
\hline 15 & 3 & 13 & 16 & 4 & 12 & 1419.8192 & 1 \\
\hline 15 & 3 & 12 & 16 & 4 & 13 & 1419.8289 & -2 \\
\hline 17 & 7 & 10 & 17 & 8 & 9 & 1419.9831 & 4 \\
\hline 10 & 4 & 6 & 11 & 5 & 7 & 1420.1941 & 0 \\
\hline 18 & 7 & 11 & 18 & 8 & 10 & 1420.3132 & 3 \\
\hline 20 & 2 & 18 & 21 & 3 & 19 & 1420.3879 & -4 \\
\hline 19 & 2 & 18 & 20 & 3 & 17 & 1420.5514 & 12 \\
\hline 19 & 7 & 12 & 19 & 8 & 11 & 1420.6574 & 1 \\
\hline
\end{tabular}

$\begin{array}{rrr}1420.8816 & -1 & 4 \\ 1421.0160 & 7 & 4\end{array}$

$1421.1513 \quad 004$

$\begin{array}{rrr}1421.3865 & 4 & 4 \\ 14 & 4\end{array}$

$1421.5401 \quad-6 \quad 4$

$1421.5928 \quad-1 \quad 4$

$1421.7705 \quad 15 \quad 4$

$1421.9257 \quad-7 \quad 4$

$1422.1643 \quad 8 \quad 4$

$1422.4979 \quad 6 \quad 4$

$\begin{array}{rrr}1422.5696 & 6 & 4\end{array}$

$1422.7213 \quad-5 \quad 4$

$1422.7753 \quad 1 \quad 4$

$1422.9854 \quad 4 \quad 4$

$\begin{array}{rrr}1423.0076 & -2 & 4 \\ 1423.3000 & 1 & 4\end{array}$

$1423.4119 \quad 11 \quad 4$

$1423.5897 \quad 10 \quad 4$

$\begin{array}{lll}1423.8462 & 2 & 4\end{array}$

$1423.8586 \quad-16 \quad 4$

$\begin{array}{lll}1423.9302 & -6 & 4\end{array}$

$\begin{array}{lll}1424.0377 & -1 & 4\end{array}$

$1424.1880-4 \quad 4$

$1424.2911 \quad 11 \quad 4$

$1424.3565 \quad-2 \quad 4$

$\begin{array}{rrr}4.4390 & 0 & 4\end{array}$

$\begin{array}{rrr}1424.5425 & -1 & 4 \\ 1424.6733 & 2 & 4\end{array}$

$1424.7450 \quad-5 \quad 10$

$1424.7450 \quad 26 \quad 10$

$1424.9650 \quad-1 \quad 4$

$1425.0462 \quad 0 \quad 4$

$\begin{array}{lll}1425.1037 & -13 & 4\end{array}$

$\begin{array}{lll}1425.1659 & -7 & 4\end{array}$

$\begin{array}{rrr}1425.2013 & 4 & 4\end{array}$

$1425.2869 \quad 2 \quad 4$

$1425.4528 \quad 1 \quad 4$

$1425.7197 \quad 0 \quad 4$

$\begin{array}{rrr}1425.8868 & 1 & 4 \\ 1425.9918 & 12 & 4\end{array}$

$1426.0019 \quad 2 \quad 4$

$1426.0478 \quad-4 \quad 4$

$1426.1685 \quad 1 \quad 4$

$\begin{array}{rrr}1426.2982 & 2 & 4 \\ 1426.3449 & -2 & 4\end{array}$

$\begin{array}{lll}1426.4279 & -3 & 4\end{array}$

$\begin{array}{lll}1426.6086 & 3 & 4\end{array}$

$1426.6228 \quad-5 \quad 4$

$\begin{array}{rrr}1426.9324 & 4 & 4 \\ 1427.0787 & -1 & 4\end{array}$

$\begin{array}{lll}1427.1878 & -3 & 4\end{array}$

$1427.2683 \quad-2 \quad 4$

$\begin{array}{lll}1427.3511 & -2 & 4\end{array}$

$\begin{array}{rrr}1427.6180 & 5 & 4\end{array}$

$1427.7143 \quad-3 \quad 4$

$\begin{array}{lll}1427.8178 & -11 & 4\end{array}$

$\begin{array}{rrr}1427.9788 & 5 & 4\end{array}$

$1428.0151 \quad-1 \quad 4$

$\begin{array}{lll}1428.0405 & -12 & 4\end{array}$

$1428.3513 \quad 8 \quad 4$

$\begin{array}{lll}1428.5949 & 2 & 4 \\ 1428.7342 & 7 & 4\end{array}$

$1428.7641 \quad 5 \quad 4$

$\begin{array}{lll}1428.8114 & 1 & 4\end{array}$

$1428.8326 \quad-1 \quad 4$

$\begin{array}{lll}1428.9069 & 8 & 4 \\ 1428.9309 & 7 & 4\end{array}$

$1428.9787 \quad 0 \quad 4$

$\begin{array}{rrr}1429.0247 & -1 & 4 \\ 1429.1277 & 9 & 4\end{array}$

$\begin{array}{lll}1429.4484 & 0 & 4\end{array}$

$\begin{array}{rrr}1429.5311 & 11 & 4\end{array}$ 


\begin{tabular}{|c|c|c|c|c|c|c|c|}
\hline 19 & 0 & 19 & 20 & 1 & 20 & 1437.2429 & -9 \\
\hline 9 & 4 & 5 & 9 & 5 & 4 & 1437.2616 & -1 \\
\hline 7 & 2 & 6 & 8 & 3 & 5 & 1437.3119 & 1 \\
\hline 7 & 2 & 5 & 8 & 3 & 6 & 1437.3303 & -1 \\
\hline 23 & 2 & 22 & 24 & 1 & 23 & 1437.3500 & 3 \\
\hline 10 & 4 & 6 & 10 & 5 & 5 & 1437.4286 & -1 \\
\hline 28 & 3 & 26 & 29 & 2 & 27 & 1437.5469 & 15 \\
\hline 11 & 4 & 7 & 11 & 5 & 6 & 1437.6113 & 0 \\
\hline 12 & 1 & 11 & 13 & 2 & 12 & 1437.7053 & -2 \\
\hline 12 & 4 & 8 & 12 & 5 & 7 & 1437.8088 & -1 \\
\hline 13 & 4 & 9 & 13 & 5 & 8 & 1438.0212 & -1 \\
\hline 14 & 4 & 10 & 14 & 5 & 9 & 1438.2468 & -10 \\
\hline 20 & 1 & 20 & 21 & 0 & 21 & 1438.3866 & -3 \\
\hline 15 & 4 & 11 & 15 & 5 & 10 & 1438.4874 & -1 \\
\hline 10 & 1 & 10 & 11 & 2 & 9 & 1438.5876 & -7 \\
\hline 16 & 4 & 12 & 16 & 5 & 11 & 1438.7388 & 3 \\
\hline 6 & 2 & 5 & 7 & 3 & 4 & 1438.7715 & 3 \\
\hline 6 & 2 & 4 & 7 & 3 & 5 & 1438.7817 & 3 \\
\hline 22 & 2 & 21 & 23 & 1 & 22 & 1438.8357 & 6 \\
\hline 11 & 1 & 10 & 12 & 2 & 11 & 1438.9267 & -2 \\
\hline 17 & 4 & 13 & 17 & 5 & 12 & 1438.9957 & 6 \\
\hline 27 & 3 & 25 & 28 & 2 & 26 & 1439.1317 & 6 \\
\hline 18 & 4 & 14 & 18 & 5 & 13 & 1439.2179 & 20 \\
\hline 17 & 0 & 17 & 18 & 1 & 18 & 1439.2661 & -4 \\
\hline 17 & 4 & 13 & 18 & 4 & 14 & 1439.5907 & 2 \\
\hline 19 & 1 & 19 & 20 & 0 & 20 & 1439.5982 & -7 \\
\hline 19 & 4 & 15 & 19 & 5 & 14 & 1439.6662 & -26 \\
\hline 20 & 4 & 16 & 20 & 5 & 15 & 1439.9443 & -19 \\
\hline 10 & 1 & 9 & 11 & 2 & 10 & 1440.1774 & -2 \\
\hline 9 & 1 & 9 & 10 & 2 & 8 & 1440.1846 & -1 \\
\hline 10 & 6 & 4 & 9 & 7 & 3 & 1440.2051 & 3 \\
\hline 17 & 8 & 9 & 16 & 9 & 8 & 1440.2326 & -16 \\
\hline 5 & 2 & 4 & 6 & 3 & 3 & 1440.2451 & 2 \\
\hline 5 & 2 & 3 & 6 & 3 & 4 & 1440.2508 & 9 \\
\hline 21 & 4 & 17 & 21 & 5 & 16 & 1440.2565 & -19 \\
\hline 16 & 0 & 16 & 17 & 1 & 17 & 1440.3073 & -5 \\
\hline 21 & 2 & 20 & 22 & 1 & 21 & 1440.3387 & 4 \\
\hline 16 & 2 & 14 & 17 & 2 & 15 & 1440.4019 & -5 \\
\hline 22 & 4 & 18 & 22 & 5 & 17 & 1440.5856 & -20 \\
\hline 26 & 3 & 24 & 27 & 2 & 25 & 1440.7155 & 9 \\
\hline 18 & 1 & 18 & 19 & 0 & 19 & 1440.8413 & -4 \\
\hline 16 & 4 & 12 & 17 & 4 & 13 & 1440.9097 & 10 \\
\hline 23 & 4 & 19 & 23 & 5 & 18 & 1440.9274 & -29 \\
\hline 16 & 5 & 11 & 17 & 5 & 12 & 1440.9564 & 5 \\
\hline 17 & 1 & 17 & 18 & 1 & 18 & 1440.9659 & 16 \\
\hline 16 & 6 & 10 & 17 & 6 & 11 & 1440.9925 & 7 \\
\hline 14 & 7 & 7 & 13 & 8 & 6 & 1441.0176 & -3 \\
\hline 24 & 4 & 20 & 24 & 5 & 19 & 1441.2846 & -5 \\
\hline 15 & 0 & 15 & 16 & 1 & 16 & 1441.3703 & -5 \\
\hline 9 & 1 & 8 & 10 & 2 & 9 & 1441.4574 & -1 \\
\hline 7 & 5 & 2 & 6 & 6 & 1 & 1441.4829 & 2 \\
\hline 25 & 4 & 22 & 25 & 5 & 21 & 1441.6449 & -14 \\
\hline 25 & 4 & 21 & 25 & 5 & 20 & 1441.6495 & -19 \\
\hline 4 & 2 & 2 & 5 & 3 & 3 & 1441.7346 & -10 \\
\hline 8 & 1 & 8 & 9 & 2 & 7 & 1441.7686 & -1 \\
\hline 20 & 2 & 19 & 21 & 1 & 20 & 1441.8580 & 6 \\
\hline 26 & 4 & 23 & 26 & 5 & 22 & 1442.0204 & -12 \\
\hline 26 & 4 & 22 & 26 & 5 & 21 & 1442.0271 & -15 \\
\hline 17 & 1 & 17 & 18 & 0 & 18 & 1442.1148 & -5 \\
\hline 16 & 1 & 16 & 17 & 1 & 17 & 1442.1347 & -4 \\
\hline 18 & 8 & 10 & 17 & 9 & 9 & 1442.1511 & 13 \\
\hline 15 & 4 & 11 & 16 & 4 & 12 & 1442.2317 & 4 \\
\hline 15 & 5 & 10 & 16 & 5 & 11 & 1442.2610 & 14 \\
\hline 15 & 6 & 9 & 16 & 6 & 10 & 1442.2782 & -9 \\
\hline 25 & 3 & 23 & 26 & 2 & 24 & 1442.2953 & 13 \\
\hline 27 & 4 & 24 & 27 & 5 & 23 & 1442.4051 & -17 \\
\hline 27 & 4 & 23 & 27 & 5 & 22 & 1442.4167 & 4 \\
\hline 14 & 0 & 14 & 15 & 1 & 15 & 1442.4567 & -3 \\
\hline 8 & 1 & 7 & 9 & 2 & 8 & 1442.7665 & 0 \\
\hline 15 & 0 & 15 & 16 & 0 & 16 & 1442.7948 & -2 \\
\hline 28 & 4 & 25 & 28 & 5 & 24 & 1442.8003 & -12 \\
\hline 28 & 4 & 24 & 28 & 5 & 23 & 1442.8133 & -10 \\
\hline 15 & 7 & 8 & 14 & 8 & 7 & 1442.8489 & 6 \\
\hline 4 & 3 & 1 & 4 & 4 & 0 & 1443.1283 & 8 \\
\hline 29 & 4 & 26 & 29 & 5 & 25 & 1443.2042 & -10 \\
\hline 5 & 3 & 2 & 5 & 4 & 1 & 1443.2101 & 1 \\
\hline 29 & 4 & 25 & 29 & 5 & 24 & 1443.2192 & -29 \\
\hline
\end{tabular}


$\begin{array}{lllllllll}24 & 3 & 21 & 24 & 4 & 20 & 1447.6559 & -3 & 4\end{array}$

1444.5438

1444.6538
1444.6597

1444.6667

$113 \quad 1444.7044$

$16 \quad 1444.7518$

1444.7570

1444.8873

1444.8958

1444.9106

1445.0066

1445.1338

1445.1462

1445.2893

1445.3933

1445.4108

1445.4716

1445.6653

1445.6897

1445.7238

1445.7811

1445.8680

1445.9487

1445.9823

1446.0941

1446.1135

1446.2437

1446.2575

1446.2692

1446.2892
1446.3575

1446.4737

1446.4906

1446.5492

1446.5789

1446.5837

1446.6567

1446.8672

1446.9447

1446.9925

$1447.0408-13$

$1447.0601-1$

$\begin{array}{ll}1447.1641 & 13 \\ 1447.1681 & -6\end{array}$

$\begin{array}{lll}1 & 11 & 1447.1681 \\ 4 & 20 & 1447.1909\end{array}$

$\begin{array}{lll}4 & 19 & 1447.2930\end{array}$

1447.3739

$\begin{array}{rrr}0 & 14 & 1447.5021 \\ 4 & 21 & 1447.5254 \\ 5 & 7 & 1447.6196\end{array}$

1447.6196
1447.6415

11
25
11
4
25
16
24
26
9
4
11
11
26
18
21
27
10
27
12
28
10
10
10
10
10
10
23
15
28
29
3

11

$\begin{array}{lllll}3 & 23 & 25 & 4 & 22\end{array}$

$\begin{array}{rr}11 & 12 \\ 4 & 5\end{array}$

$\begin{array}{lllll}5 & 3 & 22 & 25 & 4\end{array}$

$\begin{array}{llllll}16 & 2 & 15 & 17 & 1 & 16 \\ 24 & 3 & 21 & 25 & 2 & 24\end{array}$

$\begin{array}{llll}26 & 3 & 24 & 26\end{array}$

10

23
15

15
5
5

5
29
30
3<smiles>[BH3-]</smiles><smiles>C1CCC2CCC2C1</smiles>

11

$12 \quad 1447.8960 \quad 4 \quad 4$

$\begin{array}{llll}3 & 1448.0335 & -1 & 10\end{array}$

$\begin{array}{llll}21 & 1448.0335 & 3 & 10\end{array}$

$16 \quad 1448.0554$

1448.0554
1448.1490

1448.2187

1448.2818

1448.2932

1448.3254

1448.4128

$1448.4243 \quad-3 \quad 4$

$1448.4749 \quad-2 \quad 4$

$\begin{array}{lll}1448.4749 & -2 & 4 \\ 1448.5406 & -7 & 4\end{array}$

$1448.5776 \quad 16 \quad 4$

$1448.6281 \quad 8 \quad 4$

$1448.8301 \quad-4 \quad 4$

$1448.9160 \quad-1 \quad 4$

$\begin{array}{lll}1448.9415 & 18 & 4\end{array}$

$1448.9481 \quad 11 \quad 4$

$1448.9632 \quad 7 \quad 4$

$\begin{array}{lll}1448.9969 & 3 & 4\end{array}$

$1449.0296 \quad-1 \quad 4$

$1449.0440 \quad-11 \quad 10$

$\begin{array}{lll}1449.1076 & 5 & 4\end{array}$

$1449.1719 \quad 3 \quad 4$

$\begin{array}{lll}1449.2034 & 7 & 4 \\ 1449.2511 & 0 & 4\end{array}$

$\begin{array}{lll}1449.3119 & 31 & 4\end{array}$

$\begin{array}{lll}1449.5086 & -1 & 4\end{array}$

$1449.5337 \quad 1 \quad 4$

$1449.5732 \quad-8 \quad 4$

$\begin{array}{lll}1449.5932 & 3 & 4\end{array}$

$1449.6273 \quad 4 \quad 4$

$\begin{array}{rrr}1449.6517 & 6 & 4\end{array}$

$\begin{array}{lll}1449.6847 & -18 & 10\end{array}$

$\begin{array}{lll}1449.6847 & 19 & 10\end{array}$

$\begin{array}{lll}1449.7457 & -9 & 10\end{array}$

$1449.7457 \quad 5 \quad 10$

$\begin{array}{lll}1449.7550 & 1 & 4\end{array}$

$1449.8528 \quad-10 \quad 4$

$\begin{array}{lll}1449.8712 & -1 & 4\end{array}$

$\begin{array}{rrr}1449.9769 & 6 & 4\end{array}$

$\begin{array}{lll}1450.0052 & -1 & 4\end{array}$

$1450.0796 \quad 2 \quad 4$

$1450.1003 \quad 10 \quad 4$

$\begin{array}{lll}1450.1120 & -1 & 4\end{array}$

$\begin{array}{rrr}1450.1575 & 1 & 4 \\ 1450.1843 & -4 & 4\end{array}$

$\begin{array}{rrr}1450.2308 & -4 & 4\end{array}$

$1450.2606 \quad 0 \quad 4$

$1450.3281 \quad 1 \quad 4$

$1450.3528 \quad-3 \quad 4$

$\begin{array}{rrr}1450.3810 & 9 & 4 \\ 1450.3896 & -10 & 4\end{array}$

$\begin{array}{lll}1450.4052 & -8 & 4\end{array}$

$\begin{array}{lll}1450.4211 & 1 & 4\end{array}$

$\begin{array}{lll}1450.4332 & 7 & 4\end{array}$

$1450.4577 \quad 11 \quad 4$

$\begin{array}{lll}1450.5093 & 6 & 4\end{array}$

$1450.5176 \quad-1 \quad 4$

$\begin{array}{lll}1450.5926 & 0 & 4 \\ 1450.6109 & 6 & 4\end{array}$

$1450.7266 \quad-1 \quad 4$

$\begin{array}{lll}1450.7746 & -1 & 4\end{array}$

$1450.8166 \quad-1 \quad 4$

$\begin{array}{lll}1450.9555 & -2 & 4\end{array}$

$\begin{array}{lll}1450.9662 & -1 & 4\end{array}$

$\begin{array}{lll}1451.0476 & 1 & 10 \\ 1451.0476 & 4 & 10\end{array}$

$\begin{array}{rrr}1451.0476 & 4 & 10 \\ 1451.1533 & 4 & 4\end{array}$

$\begin{array}{rrr}1451.1668 & 0 & 4 \\ 1451.2046 & -1 & 4\end{array}$ 


\begin{tabular}{|c|c|c|c|c|c|c|c|}
\hline 2 & 1 & 1 & 3 & 2 & 2 & 1451.2294 & 5 \\
\hline 25 & 4 & 22 & 26 & 3 & 23 & 1451.2653 & -8 \\
\hline 21 & 3 & 18 & 22 & 2 & 21 & 1451.3212 & -7 \\
\hline 9 & 4 & 5 & 8 & 5 & 4 & 1451.3642 & -1 \\
\hline 16 & 2 & 15 & 16 & 3 & 14 & 1451.3752 & 0 \\
\hline 15 & 2 & 13 & 15 & 3 & 12 & 1451.4741 & -2 \\
\hline 17 & 2 & 16 & 17 & 3 & 15 & 1451.5908 & 1 \\
\hline 19 & 3 & 17 & 20 & 2 & 18 & 1451.6059 & -7 \\
\hline 8 & 6 & 2 & 9 & 6 & 3 & 1451.7292 & -10 \\
\hline 16 & 2 & 14 & 16 & 3 & 13 & 1451.7641 & -3 \\
\hline 8 & 5 & 3 & 9 & 5 & 4 & 1451.8011 & 2 \\
\hline 18 & 2 & 17 & 18 & 3 & 16 & 1451.8118 & -7 \\
\hline 10 & 1 & 10 & 11 & 0 & 11 & 1451.8118 & 6 \\
\hline 8 & 4 & 4 & 9 & 4 & 5 & 1451.8507 & 0 \\
\hline 8 & 2 & 6 & 9 & 2 & 7 & 1451.8578 & 6 \\
\hline 8 & 3 & 5 & 9 & 3 & 6 & 1451.8829 & 7 \\
\hline 8 & 2 & 7 & 9 & 2 & 8 & 1451.9272 & -3 \\
\hline 13 & 5 & 8 & 12 & 6 & 7 & 1451.9719 & -1 \\
\hline 8 & 0 & 8 & 9 & 0 & 9 & 1452.0050 & 0 \\
\hline 19 & 2 & 18 & 19 & 3 & 17 & 1452.0401 & 2 \\
\hline 17 & 2 & 15 & 17 & 3 & 14 & 1452.0747 & -3 \\
\hline 6 & 0 & 6 & 7 & 1 & 7 & 1452.1319 & 1 \\
\hline 20 & 2 & 19 & 20 & 3 & 18 & 1452.2722 & 3 \\
\hline 20 & 7 & 13 & 19 & 8 & 12 & 1452.2984 & 5 \\
\hline 8 & 1 & 8 & 9 & 1 & 9 & 1452.3107 & 1 \\
\hline 18 & 2 & 16 & 19 & 1 & 19 & 1452.3435 & -3 \\
\hline 18 & 2 & 16 & 18 & 3 & 15 & 1452.4054 & -5 \\
\hline 20 & 3 & 17 & 21 & 2 & 20 & 1452.4456 & -1 \\
\hline 21 & 2 & 20 & 21 & 3 & 19 & 1452.5083 & 4 \\
\hline 24 & 4 & 21 & 25 & 3 & 22 & 1452.6138 & -6 \\
\hline 1 & 1 & 1 & 2 & 2 & 0 & 1452.7145 & -3 \\
\hline 1 & 1 & 0 & 2 & 2 & 1 & 1452.7401 & 2 \\
\hline 22 & 2 & 21 & 22 & 3 & 20 & 1452.7476 & 6 \\
\hline 19 & 2 & 17 & 19 & 3 & 16 & 1452.7563 & -3 \\
\hline 13 & 2 & 12 & 14 & 1 & 13 & 1452.7876 & -1 \\
\hline 17 & 6 & 11 & 16 & 7 & 10 & 1452.9064 & 2 \\
\hline 23 & 2 & 22 & 23 & 3 & 21 & 1452.9892 & 5 \\
\hline 7 & 1 & 6 & 8 & 1 & 7 & 1453.0832 & 14 \\
\hline 10 & 4 & 6 & 9 & 5 & 5 & 1453.0976 & 1 \\
\hline 17 & 2 & 15 & 18 & 1 & 18 & 1453.1144 & -5 \\
\hline 20 & 2 & 18 & 20 & 3 & 17 & 1453.1255 & -8 \\
\hline 24 & 4 & 20 & 25 & 3 & 23 & 1453.1763 & -18 \\
\hline 7 & 5 & 2 & 8 & 5 & 3 & 1453.2271 & -8 \\
\hline 24 & 2 & 23 & 24 & 3 & 22 & 1453.2330 & 8 \\
\hline 9 & 1 & 9 & 10 & 0 & 10 & 1453.2878 & -4 \\
\hline 7 & 2 & 5 & 8 & 2 & 6 & 1453.3181 & 9 \\
\hline 7 & 3 & 4 & 8 & 3 & 5 & 1453.3225 & 1 \\
\hline 7 & 2 & 6 & 8 & 2 & 7 & 1453.3633 & 1 \\
\hline 7 & 0 & 7 & 8 & 0 & 8 & 1453.4230 & 0 \\
\hline 5 & 0 & 5 & 6 & 1 & 6 & 1453.4791 & -3 \\
\hline 21 & 2 & 19 & 21 & 3 & 18 & 1453.5132 & -6 \\
\hline 19 & 3 & 16 & 20 & 2 & 19 & 1453.5995 & -2 \\
\hline 7 & 1 & 7 & 8 & 1 & 8 & 1453.6840 & -2 \\
\hline 26 & 2 & 25 & 26 & 3 & 24 & 1453.7229 & 7 \\
\hline 14 & 5 & 9 & 13 & 6 & 8 & 1453.7737 & -3 \\
\hline 22 & 2 & 20 & 22 & 3 & 19 & 1453.9174 & -4 \\
\hline 16 & 2 & 14 & 17 & 1 & 17 & 1453.9426 & -3 \\
\hline 23 & 4 & 20 & 24 & 3 & 21 & 1453.9525 & -21 \\
\hline 27 & 2 & 26 & 27 & 3 & 25 & 1453.9695 & 18 \\
\hline 28 & 2 & 27 & 28 & 3 & 26 & 1454.2134 & 7 \\
\hline 21 & 7 & 14 & 20 & 8 & 13 & 1454.2285 & 7 \\
\hline 23 & 2 & 21 & 23 & 3 & 20 & 1454.3361 & -5 \\
\hline 12 & 2 & 11 & 13 & 1 & 12 & 1454.3745 & -1 \\
\hline 7 & 3 & 4 & 6 & 4 & 3 & 1454.3951 & 2 \\
\hline 23 & 4 & 19 & 24 & 3 & 22 & 1454.4015 & -9 \\
\hline 29 & 2 & 28 & 29 & 3 & 27 & 1454.4594 & 24 \\
\hline 17 & 3 & 15 & 18 & 2 & 16 & 1454.6266 & -3 \\
\hline 30 & 2 & 29 & 30 & 3 & 28 & 1454.7020 & 19 \\
\hline 6 & 4 & 2 & 7 & 4 & 3 & 1454.7352 & 4 \\
\hline 24 & 2 & 22 & 24 & 3 & 21 & 1454.7678 & -3 \\
\hline 8 & 1 & 8 & 9 & 0 & 9 & 1454.7818 & -2 \\
\hline 6 & 2 & 4 & 7 & 2 & 5 & 1454.7871 & -4 \\
\hline 6 & 2 & 5 & 7 & 2 & 6 & 1454.8162 & 3 \\
\hline 15 & 2 & 13 & 16 & 1 & 16 & 1454.8254 & -7 \\
\hline 11 & 4 & 7 & 10 & 5 & 6 & 1454.8460 & 1 \\
\hline 4 & 0 & 4 & 5 & 1 & 5 & 1454.8604 & 3 \\
\hline 31 & 2 & 30 & 31 & 3 & 29 & 1454.9435 & 18 \\
\hline
\end{tabular}




\begin{tabular}{|c|c|c|c|c|c|c|c|}
\hline 20 & 4 & 17 & 21 & 3 & 18 & 1457.9553 & -21 \\
\hline 13 & 1 & 12 & 13 & 2 & 11 & 1458.0168 & -6 \\
\hline 23 & 7 & 16 & 22 & 8 & 15 & 1458.1224 & 10 \\
\hline 20 & 4 & 16 & 21 & 3 & 19 & 1458.1652 & -10 \\
\hline 14 & 1 & 13 & 14 & 2 & 12 & 1458.2827 & -2 \\
\hline 32 & 2 & 30 & 32 & 3 & 29 & 1458.3715 & 7 \\
\hline 13 & 4 & 9 & 12 & 5 & 8 & 1458.3869 & 2 \\
\hline 15 & 3 & 12 & 16 & 2 & 15 & 1458.4907 & -2 \\
\hline 15 & 1 & 14 & 15 & 2 & 13 & 1458.5490 & -3 \\
\hline 20 & 6 & 14 & 19 & 7 & 13 & 1458.5611 & 11 \\
\hline 33 & 2 & 31 & 33 & 3 & 30 & 1458.8008 & 10 \\
\hline 16 & 1 & 15 & 16 & 2 & 14 & 1458.8139 & 0 \\
\hline 11 & 2 & 9 & 12 & 1 & 12 & 1458.8739 & -1 \\
\hline 17 & 1 & 16 & 17 & 2 & 15 & 1459.0743 & 0 \\
\hline 9 & 2 & 8 & 10 & 1 & 9 & 1459.1509 & 4 \\
\hline 6 & 2 & 4 & 5 & 3 & 3 & 1459.1617 & 6 \\
\hline 3 & 1 & 2 & 4 & 1 & 3 & 1459.1895 & 4 \\
\hline 1 & 0 & 1 & 2 & 1 & 2 & 1459.2042 & 1 \\
\hline 34 & 2 & 32 & 34 & 3 & 31 & 1459.2169 & 6 \\
\hline 3 & 0 & 3 & 4 & 0 & 4 & 1459.3054 & 3 \\
\hline 19 & 4 & 16 & 20 & 3 & 17 & 1459.3135 & -19 \\
\hline 18 & 1 & 17 & 18 & 2 & 16 & 1459.3282 & -1 \\
\hline 5 & 1 & 5 & 6 & 0 & 6 & 1459.3432 & 0 \\
\hline 19 & 4 & 15 & 20 & 3 & 18 & 1459.4710 & -19 \\
\hline 10 & 3 & 7 & 9 & 4 & 6 & 1459.5337 & -3 \\
\hline 19 & 1 & 18 & 19 & 2 & 17 & 1459.5740 & 0 \\
\hline 30 & 0 & 30 & 30 & 1 & 29 & 1459.6573 & -7 \\
\hline 14 & 3 & 11 & 15 & 2 & 14 & 1459.7758 & -6 \\
\hline 29 & 0 & 29 & 29 & 1 & 28 & 1459.8098 & -15 \\
\hline 20 & 1 & 19 & 20 & 2 & 18 & 1459.8098 & 1 \\
\hline 28 & 0 & 28 & 28 & 1 & 27 & 1459.9715 & -2 \\
\hline 10 & 2 & 8 & 11 & 1 & 11 & 1460.0055 & 1 \\
\hline 21 & 1 & 20 & 21 & 2 & 19 & 1460.0343 & 1 \\
\hline 24 & 7 & 17 & 23 & 8 & 16 & 1460.0837 & -1 \\
\hline 27 & 0 & 27 & 27 & 1 & 26 & 1460.1367 & -3 \\
\hline 14 & 4 & 10 & 13 & 5 & 9 & 1460.1784 & 3 \\
\hline 22 & 1 & 21 & 22 & 2 & 20 & 1460.2463 & -1 \\
\hline 26 & 0 & 26 & 26 & 1 & 25 & 1460.3043 & -6 \\
\hline 23 & 1 & 22 & 23 & 2 & 21 & 1460.4455 & 4 \\
\hline 25 & 0 & 25 & 25 & 1 & 24 & 1460.4718 & -12 \\
\hline 18 & 4 & 15 & 19 & 3 & 16 & 1460.4894 & 24 \\
\hline 13 & 3 & 11 & 14 & 2 & 12 & 1460.5467 & 1 \\
\hline 18 & 4 & 14 & 19 & 3 & 17 & 1460.6067 & 27 \\
\hline 24 & 1 & 23 & 24 & 2 & 22 & 1460.6327 & -3 \\
\hline 24 & 0 & 24 & 24 & 1 & 23 & 1460.6392 & 0 \\
\hline 3 & 1 & 2 & 2 & 2 & 1 & 1460.7203 & 3 \\
\hline 0 & 0 & 0 & 1 & 1 & 1 & 1460.7203 & 4 \\
\hline 8 & 2 & 7 & 9 & 1 & 8 & 1460.7451 & -2 \\
\hline 25 & 1 & 24 & 25 & 2 & 23 & 1460.8017 & -1 \\
\hline 23 & 0 & 23 & 23 & 1 & 22 & 1460.8017 & 3 \\
\hline 7 & 2 & 6 & 6 & 3 & 3 & 1460.8278 & 5 \\
\hline 7 & 2 & 5 & 6 & 3 & 4 & 1460.8459 & 7 \\
\hline 4 & 1 & 4 & 5 & 0 & 5 & 1460.8845 & 5 \\
\hline 22 & 0 & 22 & 22 & 1 & 21 & 1460.9577 & -1 \\
\hline 26 & 1 & 25 & 26 & 2 & 24 & 1460.9577 & 2 \\
\hline 13 & 3 & 10 & 14 & 2 & 13 & 1461.0852 & 4 \\
\hline 27 & 1 & 26 & 27 & 2 & 25 & 1461.0991 & 9 \\
\hline 21 & 0 & 21 & 21 & 1 & 20 & 1461.1067 & -2 \\
\hline 18 & 5 & 13 & 17 & 6 & 12 & 1461.1202 & 0 \\
\hline 9 & 2 & 7 & 10 & 1 & 10 & 1461.1811 & 1 \\
\hline 28 & 1 & 27 & 28 & 2 & 26 & 1461.2244 & 9 \\
\hline 20 & 0 & 20 & 20 & 1 & 19 & 1461.2470 & -3 \\
\hline 11 & 3 & 8 & 10 & 4 & 7 & 1461.2768 & -7 \\
\hline 29 & 1 & 28 & 29 & 2 & 27 & 1461.3343 & 11 \\
\hline 19 & 0 & 19 & 19 & 1 & 18 & 1461.3778 & -3 \\
\hline 30 & 1 & 29 & 30 & 2 & 28 & 1461.4282 & 11 \\
\hline 18 & 0 & 18 & 18 & 1 & 17 & 1461.4982 & -5 \\
\hline 31 & 1 & 30 & 31 & 2 & 29 & 1461.5066 & 13 \\
\hline 32 & 1 & 31 & 32 & 2 & 30 & 1461.5697 & 19 \\
\hline 17 & 0 & 17 & 17 & 1 & 16 & 1461.6084 & -3 \\
\hline 16 & 0 & 16 & 16 & 1 & 15 & 1461.7077 & -4 \\
\hline 15 & 0 & 15 & 15 & 1 & 14 & 1461.7966 & -3 \\
\hline 14 & 0 & 14 & 14 & 1 & 13 & 1461.8750 & -5 \\
\hline 17 & 4 & 14 & 18 & 3 & 15 & 1461.8832 & 19 \\
\hline 13 & 0 & 13 & 13 & 1 & 12 & 1461.9444 & -1 \\
\hline 17 & 4 & 13 & 18 & 3 & 16 & 1461.9678 & 10 \\
\hline 15 & 4 & 11 & 14 & 5 & 10 & 1461.9825 & 2 \\
\hline
\end{tabular}




\begin{tabular}{|c|c|c|c|c|c|c|c|c|}
\hline 6 & 1 & 5 & 5 & 2 & 4 & 1465.8453 & 1 & 4 \\
\hline 7 & 3 & 4 & 7 & 3 & 5 & 1465.8644 & 0 & 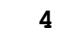 \\
\hline 19 & 5 & 15 & 20 & 4 & 16 & 1465.8778 & 12 & 4 \\
\hline 19 & 5 & 14 & 20 & 4 & 17 & 1465.8818 & 1 & \\
\hline 9 & 7 & 2 & 9 & 7 & 3 & 1465.9050 & -13 & \\
\hline 14 & 4 & 11 & 15 & 3 & 12 & 1465.9352 & -2 & 4 \\
\hline 10 & 2 & 9 & 9 & 3 & 6 & 1465.9352 & 0 & \\
\hline 8 & 4 & 4 & 8 & 4 & 5 & 1465.9562 & 1 & \\
\hline 14 & 4 & 10 & 15 & 3 & 13 & 1465.9651 & 1 & \\
\hline 10 & 8 & 2 & 10 & 8 & 3 & 1465.9966 & -14 & \\
\hline 10 & 2 & 8 & 9 & 3 & 7 & 1466.0066 & -3 & \\
\hline 9 & 5 & 4 & 9 & 5 & 5 & 1466.0596 & 3 & \\
\hline 10 & 7 & 3 & 10 & 7 & 4 & 1466.1024 & 6 & \\
\hline 9 & 3 & 6 & 9 & 3 & 7 & 1466.1359 & 5 & \\
\hline 10 & 6 & 4 & 10 & 6 & 5 & 1466.1777 & -3 & \\
\hline 11 & 8 & 3 & 11 & 8 & 4 & 1466.2315 & -13 & 10 \\
\hline 10 & 5 & 5 & 10 & 5 & 6 & 1466.2315 & 3 & $\perp$ \\
\hline 24 & 6 & 18 & 23 & 7 & 17 & 1466.2603 & 3 & \\
\hline 24 & 6 & 19 & 25 & 5 & 20 & 1466.2707 & 8 & \\
\hline 5 & 2 & 3 & 6 & 1 & 6 & 1466.2939 & 0 & \\
\hline 9 & 3 & 7 & 10 & 2 & 8 & 1466.3822 & 1 & \\
\hline 12 & 9 & 3 & 12 & 9 & 4 & 1466.3972 & 12 & \\
\hline 11 & 5 & 6 & 11 & 5 & 7 & 1466.4194 & 6 & \\
\hline 9 & 3 & 6 & 10 & 2 & 9 & 1466.5320 & 1 & \\
\hline 14 & 3 & 12 & 13 & 4 & 9 & 1466.5907 & 3 & \\
\hline 14 & 3 & 11 & 13 & 4 & 10 & 1466.5982 & 16 & \\
\hline 13 & 9 & 4 & 13 & 9 & 5 & 1466.6976 & -17 & \\
\hline 21 & 5 & 16 & 20 & 6 & 15 & 1466.7615 & 1 & \\
\hline 13 & 5 & 8 & 13 & 5 & 9 & 1466.8403 & 6 & \\
\hline 7 & 1 & 7 & 6 & 2 & 4 & 1466.8896 & -7 & \\
\hline 4 & 2 & 3 & 5 & 1 & 4 & 1467.1220 & -20 & 1 \\
\hline 3 & 0 & 3 & 2 & 1 & 2 & 1467.1220 & 8 & 10 \\
\hline 18 & 5 & 14 & 19 & 4 & 15 & 1467.1583 & 18 & \\
\hline 13 & 4 & 10 & 14 & 3 & 11 & 1467.2988 & 6 & \\
\hline 13 & 4 & 9 & 14 & 3 & 12 & 1467.3177 & -3 & \\
\hline 18 & 4 & 14 & 17 & 5 & 13 & 1467.4039 & 21 & \\
\hline 23 & 6 & 18 & 24 & 5 & 19 & 1467.4743 & 3 & \\
\hline 7 & 1 & 6 & 6 & 2 & 5 & 1467.6096 & 4 & \\
\hline 11 & 2 & 10 & 10 & 3 & 7 & 1467.6613 & -3 & \\
\hline 4 & 2 & 2 & 5 & 1 & 5 & 1467.6657 & -25 & \\
\hline 11 & 2 & 9 & 10 & 3 & 8 & 1467.7658 & 0 & \\
\hline 8 & 3 & 6 & 9 & 2 & 7 & 1467.8432 & -1 & \\
\hline 8 & 3 & 5 & 9 & 2 & 8 & 1467.9438 & 3 & \\
\hline 28 & 7 & 21 & 27 & 8 & 20 & 1468.0246 & 9 & \\
\hline 25 & 6 & 19 & 24 & 7 & 18 & 1468.2107 & 5 & \\
\hline 15 & 3 & 13 & 14 & 4 & 10 & 1468.3886 & -5 & \\
\hline 15 & 3 & 12 & 14 & 4 & 11 & 1468.3982 & -3 & \\
\hline 17 & 5 & 13 & 18 & 4 & 14 & 1468.4477 & 8 & \\
\hline 8 & 1 & 8 & 7 & 2 & 5 & 1468.4648 & 0 & \\
\hline 22 & 5 & 17 & 21 & 6 & 16 & 1468.6643 & 2 & \\
\hline 12 & 4 & 9 & 13 & 3 & 10 & 1468.6713 & 2 & \\
\hline 12 & 4 & 8 & 13 & 3 & 11 & 1468.6838 & -1 & \\
\hline 22 & 6 & 17 & 23 & 5 & 18 & 1468.6879 & 6 & \\
\hline 1 & 1 & 0 & 1 & 0 & 1 & 1468.7067 & 1 & \\
\hline 3 & 2 & 2 & 4 & 1 & 3 & 1468.7174 & 7 & \\
\hline 2 & 1 & 1 & 2 & 0 & 2 & 1468.7635 & 1 & \\
\hline 4 & 0 & 4 & 3 & 1 & 3 & 1468.8050 & 3 & \\
\hline 3 & 1 & 2 & 3 & 0 & 3 & 1468.8494 & 2 & \\
\hline 4 & 1 & 3 & 4 & 0 & 4 & 1468.9650 & 2 & \\
\hline 5 & 1 & 4 & 5 & 0 & 5 & 1469.1115 & 2 & \\
\hline 6 & 1 & 5 & 6 & 0 & 6 & 1469.2901 & 2 & \\
\hline 7 & 3 & 5 & 8 & 2 & 6 & 1469.3104 & 4 & \\
\hline 7 & 3 & 4 & 8 & 2 & 7 & 1469.3738 & -1 & \\
\hline 8 & 1 & 7 & 7 & 2 & 6 & 1469.3998 & -12 & \\
\hline 12 & 2 & 11 & 11 & 3 & 8 & 1469.3998 & 17 & \\
\hline 19 & 4 & 15 & 18 & 5 & 14 & 1469.4150 & -24 & \\
\hline 7 & 1 & 6 & 7 & 0 & 7 & 1469.5022 & 2 & \\
\hline 12 & 2 & 10 & 11 & 3 & 9 & 1469.5451 & 0 & \\
\hline 8 & 1 & 7 & 8 & 0 & 8 & 1469.7495 & 1 & 1 \\
\hline 16 & 5 & 12 & 17 & 4 & 13 & 1469.7495 & 8 & 1 \\
\hline 21 & 6 & 16 & 22 & 5 & 17 & 1469.9110 & 6 & \\
\hline 9 & 1 & 8 & 9 & 0 & 9 & 1470.0340 & -1 & \\
\hline 11 & 4 & 8 & 12 & 3 & 9 & 1470.0550 & -2 & \\
\hline 11 & 4 & 7 & 12 & 3 & 10 & 1470.0628 & -4 & \\
\hline 26 & 6 & 20 & 25 & 7 & 19 & 1470.1701 & 7 & \\
\hline 16 & 3 & 14 & 15 & 4 & 11 & 1470.2009 & 3 & \\
\hline 16 & 3 & 13 & 15 & 4 & 12 & 1470.2143 & -2 & \\
\hline
\end{tabular}

\begin{tabular}{|c|c|c|c|c|c|c|c|}
\hline 1 & 1 & 1 & 0 & 0 & 0 & 1470.2499 & 3 \\
\hline 3 & 1 & 2 & 2 & 1 & 1 & 1470.2893 & 4 \\
\hline 10 & 1 & 9 & 10 & 0 & 10 & 1470.3581 & 2 \\
\hline 5 & 0 & 5 & 4 & 1 & 4 & 1470.5210 & 2 \\
\hline 23 & 5 & 18 & 22 & 6 & 17 & 1470.5774 & 3 \\
\hline 11 & 1 & 10 & 11 & 0 & 11 & 1470.7231 & 0 \\
\hline 6 & 3 & 3 & 7 & 2 & 6 & 1470.8221 & -4 \\
\hline 15 & 5 & 11 & 16 & 4 & 12 & 1471.0628 & 4 \\
\hline 12 & 1 & 11 & 12 & 0 & 12 & 1471.1319 & 0 \\
\hline 20 & 6 & 15 & 21 & 5 & 16 & 1471.1441 & 2 \\
\hline 13 & 2 & 12 & 12 & 3 & 9 & 1471.1441 & 3 \\
\hline 9 & 1 & 8 & 8 & 2 & 7 & 1471.2202 & -1 \\
\hline 20 & 4 & 16 & 19 & 5 & 15 & 1471.2553 & -18 \\
\hline 13 & 2 & 11 & 12 & 3 & 10 & 1471.3454 & -2 \\
\hline 10 & 4 & 7 & 11 & 3 & 8 & 1471.4513 & -2 \\
\hline 10 & 4 & 6 & 11 & 3 & 9 & 1471.4572 & 9 \\
\hline 13 & 1 & 12 & 13 & 0 & 13 & 1471.5864 & 0 \\
\hline 10 & 1 & 10 & 9 & 2 & 7 & 1471.5928 & 3 \\
\hline 25 & 7 & 19 & 26 & 6 & 20 & 1471.7518 & 15 \\
\hline 2 & 1 & 2 & 1 & 0 & 1 & 1471.8244 & 2 \\
\hline 4 & 3 & 1 & 3 & 3 & 0 & 1471.8440 & -1 \\
\hline 4 & 2 & 2 & 3 & 2 & 1 & 1471.8798 & 1 \\
\hline 4 & 1 & 4 & 3 & 1 & 3 & 1471.8832 & -3 \\
\hline 4 & 0 & 4 & 3 & 0 & 3 & 1471.9051 & -4 \\
\hline 4 & 1 & 3 & 3 & 1 & 2 & 1471.9158 & 7 \\
\hline 17 & 3 & 15 & 16 & 4 & 12 & 1472.0244 & -1 \\
\hline 17 & 3 & 14 & 16 & 4 & 13 & 1472.0454 & 8 \\
\hline 14 & 1 & 13 & 14 & 0 & 14 & 1472.0883 & -3 \\
\hline 6 & 0 & 6 & 5 & 1 & 5 & 1472.2686 & -1 \\
\hline 5 & 3 & 2 & 6 & 2 & 5 & 1472.2897 & 7 \\
\hline 14 & 5 & 10 & 15 & 4 & 11 & 1472.3891 & 4 \\
\hline 19 & 6 & 14 & 20 & 5 & 15 & 1472.3891 & 7 \\
\hline 24 & 5 & 19 & 23 & 6 & 18 & 1472.5002 & 3 \\
\hline 15 & 1 & 14 & 15 & 0 & 15 & 1472.6402 & -2 \\
\hline 9 & 4 & 6 & 10 & 3 & 7 & 1472.8627 & 17 \\
\hline 14 & 2 & 13 & 13 & 3 & 10 & 1472.8969 & -3 \\
\hline 24 & 7 & 18 & 25 & 6 & 19 & 1472.9196 & 22 \\
\hline 10 & 1 & 9 & 9 & 2 & 8 & 1473.0672 & 2 \\
\hline 21 & 4 & 17 & 20 & 5 & 16 & 1473.1289 & -22 \\
\hline 11 & 1 & 11 & 10 & 2 & 8 & 1473.1403 & 0 \\
\hline 14 & 2 & 12 & 13 & 3 & 11 & 1473.1679 & -3 \\
\hline 16 & 1 & 15 & 16 & 0 & 16 & 1473.2433 & 0 \\
\hline 3 & 1 & 3 & 2 & 0 & 2 & 1473.4026 & 3 \\
\hline 5 & 4 & 1 & 4 & 4 & 0 & 1473.4422 & -7 \\
\hline 5 & 3 & 2 & 4 & 3 & 1 & 1473.4935 & 4 \\
\hline 5 & 2 & 3 & 4 & 2 & 2 & 1473.5266 & -1 \\
\hline 5 & 1 & 5 & 4 & 1 & 4 & 1473.5399 & 2 \\
\hline 5 & 0 & 5 & 4 & 0 & 4 & 1473.5515 & -1 \\
\hline 5 & 1 & 4 & 4 & 1 & 3 & 1473.5515 & 1 \\
\hline 18 & 6 & 13 & 19 & 5 & 14 & 1473.6453 & 6 \\
\hline 13 & 5 & 9 & 14 & 4 & 10 & 1473.7286 & 2 \\
\hline 4 & 3 & 2 & 5 & 2 & 3 & 1473.7641 & 17 \\
\hline 18 & 3 & 16 & 17 & 4 & 13 & 1473.8602 & -1 \\
\hline 18 & 3 & 15 & 17 & 4 & 14 & 1473.8888 & 1 \\
\hline 17 & 1 & 16 & 17 & 0 & 17 & 1473.8985 & -1 \\
\hline 7 & 0 & 7 & 6 & 1 & 6 & 1474.0478 & 1 \\
\hline 23 & 7 & 17 & 24 & 6 & 18 & 1474.0948 & 9 \\
\hline 28 & 6 & 22 & 27 & 7 & 21 & 1474.1135 & 9 \\
\hline 8 & 4 & 5 & 9 & 3 & 6 & 1474.2854 & 9 \\
\hline 25 & 5 & 20 & 24 & 6 & 19 & 1474.4321 & 4 \\
\hline 18 & 1 & 17 & 18 & 0 & 18 & 1474.6073 & 1 \\
\hline 15 & 2 & 14 & 14 & 3 & 11 & 1474.6569 & 0 \\
\hline 12 & 1 & 12 & 11 & 2 & 9 & 1474.6735 & -2 \\
\hline 17 & 6 & 12 & 18 & 5 & 13 & 1474.9136 & 4 \\
\hline 11 & 1 & 10 & 10 & 2 & 9 & 1474.9407 & -4 \\
\hline 4 & 1 & 4 & 3 & 0 & 3 & 1474.9845 & 3 \\
\hline 15 & 2 & 13 & 14 & 3 & 12 & 1475.0134 & -3 \\
\hline 22 & 4 & 18 & 21 & 5 & 17 & 1475.0197 & -18 \\
\hline 7 & 2 & 5 & 7 & 1 & 6 & 1475.0352 & -4 \\
\hline 9 & 2 & 7 & 9 & 1 & 8 & 1475.0352 & -14 \\
\hline 4 & 2 & 2 & 4 & 1 & 3 & 1475.0539 & 5 \\
\hline 11 & 2 & 9 & 11 & 1 & 10 & 1475.0615 & -4 \\
\hline 3 & 2 & 1 & 3 & 1 & 2 & 1475.0615 & 16 \\
\hline 12 & 5 & 8 & 13 & 4 & 9 & 1475.0821 & 3 \\
\hline 12 & 2 & 10 & 12 & 1 & 11 & 1475.0887 & 1 \\
\hline 6 & 4 & 2 & 5 & 4 & 1 & 1475.1110 & 1 \\
\hline 13 & 2 & $1 \overline{1}$ & 13 & 1 & 12 & 1475.1273 & -2 \\
\hline
\end{tabular}




17

$\begin{array}{lllllllll}13 & 1 & 12 & 12 & 2 & 11 & 1478.7706 & -1 & 4\end{array}$

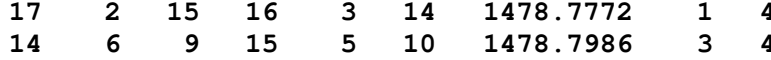
$\begin{array}{lllllllll}24 & 4 & 20 & 23 & 5 & 19 & 1478.8362 & -36 & 4\end{array}$ $\begin{array}{rrrrrrrrr}19 & 7 & 13 & 20 & 6 & 14 & 1478.9071 & 3 & 4\end{array}$ $\begin{array}{lllllllll}23 & 1 & 22 & 23 & 0 & 23 & 1478.9484 & 2 & 4\end{array}$ $\begin{array}{lllllllll}28 & 2 & 26 & 28 & 1 & 27 & 1479.0772 & -4 & 4 \\ 15 & 1 & 15 & 14 & 2 & 12 & 1479.1568 & -1 & 4\end{array}$ $\begin{array}{lllllllll}15 & 2 & 14 & 15 & 1 & 15 & 1479.2150 & 2 & 4\end{array}$ $\begin{array}{rrrrrrrrr}9 & 5 & 5 & 10 & 4 & 6 & 1479.2298 & -4 & 4 \\ 21 & 3 & 19 & 20 & 4 & 16 & 1479.4316 & -3 & 4\end{array}$ $\begin{array}{lllllllll}21 & 3 & 18 & 20 & 4 & 17 & 1479.5049 & -2 & 4\end{array}$ $\begin{array}{rrrrrrrrr}10 & 0 & 10 & 9 & 1 & 9 & 1479.5633 & -1 & 4\end{array}$ $\begin{array}{lllllllll}29 & 2 & 27 & 29 & 1 & 28 & 1479.6943 & -1 & 4\end{array}$

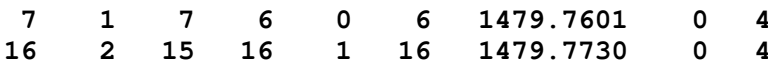




\begin{tabular}{|c|c|c|c|}
\hline 10 & 3 & 7 & 10 \\
\hline 29 & 5 & 24 & 28 \\
\hline 11 & 3 & 8 & 11 \\
\hline 10 & 3 & 8 & 10 \\
\hline 12 & 3 & 9 & 12 \\
\hline 11 & 3 & 9 & 11 \\
\hline 13 & 3 & 10 & 13 \\
\hline 19 & 2 & 17 & 18 \\
\hline 16 & 7 & 10 & 17 \\
\hline 26 & 4 & 22 & 25 \\
\hline 14 & 3 & 11 & 14 \\
\hline 15 & 1 & 14 & 14 \\
\hline 12 & 3 & 10 & 12 \\
\hline 11 & 6 & 6 & 12 \\
\hline 15 & 3 & 12 & 15 \\
\hline 16 & 3 & 13 & 16 \\
\hline 5 & 2 & 4 & 4 \\
\hline 9 & 1 & 9 & 8 \\
\hline 13 & 3 & 11 & 13 \\
\hline 17 & 3 & 14 & 17 \\
\hline 21 & 2 & 20 & 21 \\
\hline 18 & 3 & 15 & 18 \\
\hline 23 & 3 & 21 & 22 \\
\hline 19 & 3 & 16 & 19 \\
\hline 14 & 3 & 12 & 14 \\
\hline 20 & 3 & 17 & 20 \\
\hline 27 & 1 & 26 & 27 \\
\hline 23 & 3 & 20 & 22 \\
\hline 5 & 2 & 3 & 4 \\
\hline 21 & 3 & 18 & 21 \\
\hline 12 & 0 & 12 & 11 \\
\hline 22 & 3 & 19 & 22 \\
\hline 18 & 1 & 18 & 17 \\
\hline 23 & 3 & 20 & 23 \\
\hline 11 & 8 & 3 & 10 \\
\hline 20 & 2 & 19 & 19 \\
\hline 24 & 3 & 21 & 24 \\
\hline 15 & 3 & 13 & 15 \\
\hline 6 & 5 & 2 & 7 \\
\hline 11 & 7 & 4 & 10 \\
\hline 25 & 3 & 22 & 25 \\
\hline 26 & 3 & 23 & 26 \\
\hline 11 & 1 & 10 & 10 \\
\hline 11 & 6 & 5 & 10 \\
\hline 27 & 3 & 24 & 27 \\
\hline 11 & 5 & 6 & 10 \\
\hline 28 & 3 & 25 & 28 \\
\hline 11 & 3 & 8 & 10 \\
\hline 11 & 2 & 10 & 10 \\
\hline 29 & 3 & 26 & 29 \\
\hline 11 & 0 & 11 & 10 \\
\hline 16 & 3 & 14 & 16 \\
\hline 22 & 2 & 21 & 22 \\
\hline 11 & 1 & 11 & 10 \\
\hline 15 & 7 & 9 & 16 \\
\hline 17 & 3 & 15 & 17 \\
\hline 10 & 6 & 5 & 11 \\
\hline 30 & 5 & 25 & 29 \\
\hline 18 & 3 & 16 & 18 \\
\hline 28 & 1 & 27 & 28 \\
\hline 6 & 2 & 5 & 5 \\
\hline 10 & 1 & 10 & 9 \\
\hline 20 & 2 & 18 & 19 \\
\hline 27 & 4 & 23 & 26 \\
\hline 23 & 2 & 22 & 23 \\
\hline 16 & 1 & 15 & 15 \\
\hline 19 & 1 & 19 & 18 \\
\hline 19 & 3 & 17 & 19 \\
\hline 24 & 3 & 22 & 23 \\
\hline 6 & 2 & 4 & 5 \\
\hline 20 & 3 & 18 & 20 \\
\hline 14 & 7 & 8 & 15 \\
\hline 21 & 2 & 20 & 20 \\
\hline 24 & 3 & 21 & 23 \\
\hline 12 & 8 & 4 & 11 \\
\hline 12 & 1 & 11 & 11 \\
\hline 13 & 0 & 13 & 12 \\
\hline
\end{tabular}

\begin{tabular}{|c|c|c|c|}
\hline 2 & 8 & 1482.2165 & 1 \\
\hline 6 & 23 & 1482.2370 & -3 \\
\hline 2 & 9 & 1482.3406 & 1 \\
\hline 2 & 9 & 1482.3644 & -6 \\
\hline 2 & 10 & 1482.4653 & -1 \\
\hline 2 & 10 & 1482.5537 & -1 \\
\hline 2 & 11 & 1482.5889 & 0 \\
\hline 3 & 16 & 1482.6424 & -4 \\
\hline 6 & 11 & 1482.6494 & 6 \\
\hline 5 & 21 & 1482.7006 & -11 \\
\hline 2 & 12 & 1482.7082 & -7 \\
\hline 0 & 13 & 1482.7082 & 8 \\
\hline 2 & 11 & 1482.7616 & -2 \\
\hline 5 & 7 & 1482.8142 & 0 \\
\hline 2 & 13 & 1482.8234 & -3 \\
\hline 2 & 14 & 1482.9313 & -2 \\
\hline 1 & 3 & 1482.9694 & 5 \\
\hline 0 & 8 & 1482.9803 & 0 \\
\hline 2 & 12 & 1482.9893 & -1 \\
\hline 2 & 15 & 1483.0313 & -1 \\
\hline 1 & 21 & 1483.0987 & 4 \\
\hline 2 & 16 & 1483.1221 & -2 \\
\hline 4 & 18 & 1483.1924 & -1 \\
\hline 2 & 17 & 1483.2042 & 1 \\
\hline$?$ & 13 & 1483.2372 & -1 \\
\hline 2 & 18 & 1483.2765 & -2 \\
\hline 0 & 27 & 1483.3146 & 5 \\
\hline 4 & 19 & 1483.3213 & 3 \\
\hline 1 & 4 & 1483.3355 & 2 \\
\hline 2 & 19 & 1483.3401 & -8 \\
\hline 1 & 11 & 1483.3788 & 0 \\
\hline 2 & 20 & 1483.3972 & -4 \\
\hline 2 & 15 & 1483.4045 & 1 \\
\hline 2 & 21 & 1483.4481 & -2 \\
\hline 8 & 2 & 1483.4558 & -1 \\
\hline 3 & 16 & 1483.4880 & 2 \\
\hline 2 & 22 & 1483.4947 & -2 \\
\hline 2 & 14 & 1483.5057 & -1 \\
\hline 4 & 3 & 1483.5200 & 1 \\
\hline 7 & 3 & 1483.5397 & -9 \\
\hline 2 & 23 & 1483.5397 & 1 \\
\hline 2 & 24 & 1483.5838 & -12 \\
\hline 1 & 9 & 1483.5838 & 8 \\
\hline 6 & 4 & 1483.6084 & 4 \\
\hline 2 & 25 & 1483.6334 & -5 \\
\hline 5 & 5 & 1483.6531 & -4 \\
\hline 2 & 26 & 1483.6876 & -18 \\
\hline 3 & 7 & 1483.7067 & 7 \\
\hline 2 & 9 & 1483.7369 & 0 \\
\hline 2 & 27 & 1483.7541 & -8 \\
\hline 0 & 10 & 1483.7869 & 3 \\
\hline 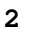 & 15 & 1483.7955 & -2 \\
\hline 1 & 22 & 1483.8695 & 5 \\
\hline 1 & 10 & 1483.9150 & -3 \\
\hline 6 & 10 & 1483.9285 & 6 \\
\hline 2 & 16 & 1484.1075 & -1 \\
\hline 5 & 6 & 1484.1852 & 9 \\
\hline 6 & 24 & 1484.2009 & -35 \\
\hline 2 & 17 & 1484.4420 & 0 \\
\hline 0 & 28 & 1484.5141 & 12 \\
\hline 1 & 4 & 1484.5418 & 0 \\
\hline 0 & 9 & 1484.6057 & -1 \\
\hline 3 & 17 & 1484.6159 & 0 \\
\hline 5 & 22 & 1484.6462 & -16 \\
\hline 1 & 23 & 1484.6749 & 7 \\
\hline 2 & 14 & 1484.7154 & 0 \\
\hline 2 & 16 & 1484.7561 & -2 \\
\hline 2 & 18 & 1484.7998 & 0 \\
\hline 4 & 19 & 1485.0846 & 5 \\
\hline 1 & 5 & 1485.0939 & 1 \\
\hline 2 & 19 & 1485.1815 & -1 \\
\hline 6 & 9 & 1485.2351 & -3 \\
\hline 3 & 17 & 1485.2462 & 3 \\
\hline 4 & 20 & 1485.2511 & -2 \\
\hline 8 & 3 & 1485.2731 & -1 \\
\hline 1 & 10 & 1485.2921 & 1 \\
\hline 1 & 12 & 1485.3241 & -3 \\
\hline
\end{tabular}

12
12
12
12
12
2
12
2

12
12
12
12
12
24
12

12

2

2

20

1

2

28

21
17

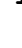

2

25

22
13

13

13
13

13

13

13

13

1

14
26

25

$\begin{array}{rrr}6 & 6 & 11 \\ 5 & 7 & 11 \\ 2 & 10 & 11 \\ 4 & 8 & 11 \\ 3 & 9 & 11 \\ 2 & 11 & 11 \\ 2 & 23 & 24 \\ 0 & 12 & 11 \\ 6 & 4 & 10 \\ 3 & 19 & 21 \\ 1 & 12 & 11 \\ 1 & 28 & 29 \\ 3 & 20 & 22 \\ 1 & 20 & 19 \\ 2 & 6 & 6 \\ 1 & 11 & 10\end{array}$

2425

2123

$24 \quad 27$

1920

$\begin{array}{rrr}16 & 16 \\ 2 & 5 & 6\end{array}$

$\begin{array}{rr}22 & 24 \\ 3 & 9\end{array}$

2324

\begin{tabular}{ll}
21 & 21 \\
\hline 12 & 12
\end{tabular}

$\begin{array}{lll}1 & 12 & 12\end{array}$

$\begin{array}{lll}9 & 4 & 12 \\ 7 & 6 & 12\end{array}$

$5 \quad 12$

$\begin{array}{rrr}6 & 11 & 12\end{array}$

$\begin{array}{rr}72 & 12 \\ 22\end{array}$

812

$\begin{array}{rr}9 & 12 \\ 10 & 12\end{array}$

$\begin{array}{ll}10 & 12 \\ 12 & 12\end{array}$

1413

2526

32325

$\begin{array}{rrr}1 & 13 & 12 \\ 2 & 7 & 7\end{array}$

$\begin{array}{rrr}7 & 6 & 13\end{array}$

$810 \quad 18$

$\begin{array}{rrr}3 & 2 & 3 \\ 1 & 12 & 11\end{array}$

$\begin{array}{rrr}1 & 12 & 11 \\ 4 & 0 & 4 \\ 3 & 24 & 26 \\ 4 & 1 & 5\end{array}$

$\begin{array}{rrr}4 & 1 & 5 \\ 4 & 2 & 6 \\ 2 & 26 & 27\end{array}$

$\begin{array}{rrr}2 & 26 & 27 \\ 4 & 3 & 7 \\ 4 & 4 & 8 \\ 4 & 5 & 9\end{array}$

$\begin{array}{rrr}4 & 5 & 9 \\ & 25 & 28\end{array}$

$3 \quad 25 \quad 27$

$\begin{array}{llll}20 & 21 & 3 & 19\end{array}$

$$
\begin{array}{rrr}
4 & 6 & 10 \\
2 & 6 & 7 \\
2 & 22 & 22
\end{array}
$$$$
1 \quad 13 \quad 13
$$$$
\begin{array}{lll}
1 & 17 & 17
\end{array}
$$$$
\begin{array}{lll}
4 & 7 & 11
\end{array}
$$$$
\begin{array}{lll}
2 & 12 & 13
\end{array}
$$$$
\begin{array}{lll}
9 & 5 & 13 \\
8 & 6 & 13
\end{array}
$$$$
\begin{array}{lll}
8 & 6 & 13 \\
6 & 8 & 13
\end{array}
$$$$
4 \quad 10 \quad 13
$$$$
\begin{array}{lll}
7 & 7 & 13 \\
4 & 9 & 12
\end{array}
$$$$
\begin{array}{lll}
4 & 9 & 12
\end{array}
$$$$
\begin{array}{lll}
7 & 23 & 25
\end{array}
$$$$
0 \quad 14 \quad 13
$$$$
\begin{array}{lll}
3 & 26 & 28 \\
2 & 27 & 28
\end{array}
$$

\begin{tabular}{|c|c|c|c|}
\hline 5 & 1485.3887 & 3 & 4 \\
\hline 6 & 1485.4210 & -10 & 10 \\
\hline 9 & 1485.4210 & 9 & 10 \\
\hline 7 & 1485.4449 & 1 & 4 \\
\hline 8 & 1485.4587 & 10 & 4 \\
\hline 10 & 1485.4924 & -3 & 4 \\
\hline 24 & 1485.5138 & 4 & 4 \\
\hline 11 & 1485.5587 & -1 & 4 \\
\hline 5 & 1485.5713 & 4 & 4 \\
\hline 20 & 1485.5883 & 2 & 4 \\
\hline 11 & 1485.7164 & -2 & 4 \\
\hline 29 & 1485.7506 & 11 & 4 \\
\hline 21 & 1486.0201 & 3 & 4 \\
\hline 17 & 1486.0743 & 12 & 4 \\
\hline 5 & 1486.1125 & -1 & 4 \\
\hline 10 & 1486.2441 & 2 & 4 \\
\hline 25 & 1486.3872 & 11 & . \\
\hline 22 & 1486.4779 & 2 & 4 \\
\hline 23 & 1486.6017 & -19 & 4 \\
\hline 18 & 1486.6163 & -3 & 4 \\
\hline 15 & 1486.7494 & 0 & A \\
\hline 6 & 1486.8894 & 2 & 4 \\
\hline 23 & 1486.9626 & 4 & 4 \\
\hline 4 & 1486.9740 & -4 & 4 \\
\hline 20 & 1486.9828 & 10 & 4 \\
\hline 18 & 1486.9962 & 7 & 4 \\
\hline 11 & 1487.0124 & 0 & 4 \\
\hline 3 & 1487.0443 & -5 & 4 \\
\hline 5 & 1487.0972 & 7 & 4 \\
\hline 4 & 1487.1079 & 3 & 4 \\
\hline 10 & 1487.1584 & -5 & 4 \\
\hline 6 & 1487.1845 & 2 & 4 \\
\hline 21 & 1487.1972 & 0 & 4 \\
\hline 7 & 1487.2052 & 1 & 4 \\
\hline 8 & 1487.2179 & 3 & 4 \\
\hline 9 & 1487.2230 & 9 & 4 \\
\hline 11 & 1487.2640 & 1 & 4 \\
\hline 13 & 1487.2930 & -4 & 10 \\
\hline 26 & 1487.2930 & 12 & 10 \\
\hline 24 & 1487.4745 & 4 & 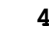 \\
\hline 12 & 1487.5378 & -4 & 4 \\
\hline 6 & 1487.6816 & 1 & 4 \\
\hline 7 & 1487.8037 & -1 & 4 \\
\hline 11 & 1487.8145 & 8 & 4 \\
\hline 1 & 1487.8800 & 9 & 4 \\
\hline 11 & 1487.8964 & -2 & 4 \\
\hline 1 & 1487.9672 & 0 & 4 \\
\hline 25 & 1488.0147 & 7 & 4 \\
\hline 2 & 1488.0503 & 0 & 4 \\
\hline 3 & 1488.1494 & 0 & 4 \\
\hline 27 & 1488.2309 & 11 & 4 \\
\hline 4 & 1488.2642 & 1 & 4 \\
\hline 5 & 1488.3943 & 4 & 4 \\
\hline 6 & 1488.5392 & 8 & 4 \\
\hline 24 & 1488.5678 & -10 & 4 \\
\hline 26 & 1488.5830 & 6 & 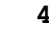 \\
\hline 19 & 1488.6451 & -4 & 4 \\
\hline 7 & 1488.6982 & 13 & 4 \\
\hline 7 & 1488.7227 & 0 & 4 \\
\hline 19 & 1488.7345 & 6 & 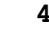 \\
\hline 12 & 1488.7444 & 0 & 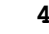 \\
\hline 16 & 1488.8088 & -1 & 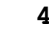 \\
\hline 8 & 1488.8692 & 5 & 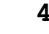 \\
\hline 11 & 1488.9034 & -7 & 4 \\
\hline 4 & 1488.9288 & 0 & 4 \\
\hline 5 & 1488.9590 & 7 & 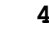 \\
\hline 7 & 1488.9963 & 13 & 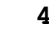 \\
\hline 9 & 1489.0037 & 2 & 4 \\
\hline 6 & 1489.0256 & -9 & 4 \\
\hline 10 & 1489.0611 & 1 & 4 \\
\hline 6 & 1489.1492 & 1 & 4 \\
\hline 22 & 1489.1599 & 8 & A \\
\hline 13 & 1489.1678 & 4 & 4 \\
\hline 27 & 1489.1809 & 9 & 4 \\
\hline 28 & 1489.2013 & 18 & 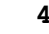 \\
\hline 7 & 1489.2488 & 1 & 4 \\
\hline 11 & 1489.2617 & 0 & \\
\hline
\end{tabular}

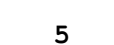




\begin{tabular}{|c|c|c|c|c|c|c|c|}
\hline 15 & 0 & 15 & 14 & 1 & 14 & 1489.2840 & -4 \\
\hline 14 & 1 & 14 & 13 & 1 & 13 & 1489.3796 & -4 \\
\hline 14 & 4 & 10 & 14 & 3 & 11 & 1489.4552 & 1 \\
\hline 14 & 4 & 11 & 14 & 3 & 12 & 1489.4748 & 0 \\
\hline 5 & 3 & 3 & 4 & 2 & 2 & 1489.5258 & 4 \\
\hline 5 & 3 & 2 & 4 & 2 & 3 & 1489.5299 & -1 \\
\hline 13 & 1 & 13 & 12 & 0 & 12 & 1489.5657 & -3 \\
\hline 15 & 4 & 11 & 15 & 3 & 12 & 1489.6702 & 2 \\
\hline 15 & 4 & 12 & 15 & 3 & 13 & 1489.6998 & 4 \\
\hline 29 & 3 & 27 & 29 & 2 & 28 & 1489.8085 & 13 \\
\hline 16 & 4 & 12 & 16 & 3 & 13 & 1489.8912 & 3 \\
\hline 16 & 4 & 13 & 16 & 3 & 14 & 1489.9341 & 3 \\
\hline 17 & 4 & 13 & 17 & 3 & 14 & 1490.1120 & 9 \\
\hline 17 & 4 & 14 & 17 & 3 & 15 & 1490.1729 & 8 \\
\hline 29 & 2 & 28 & 29 & 1 & 29 & 1490.2020 & 21 \\
\hline 18 & 4 & 14 & 18 & 3 & 15 & 1490.2901 & 19 \\
\hline 15 & 8 & 8 & 16 & 7 & 9 & 1490.2995 & 5 \\
\hline 18 & 4 & 15 & 18 & 3 & 16 & 1490.3754 & 22 \\
\hline 24 & 2 & 23 & 23 & 3 & 20 & 1490.4589 & 6 \\
\hline 30 & 3 & 28 & 30 & 2 & 29 & 1490.4655 & 10 \\
\hline 15 & 1 & 14 & 14 & 1 & 13 & 1490.4886 & 1 \\
\hline 10 & 7 & 4 & 11 & 6 & 5 & 1490.5060 & -3 \\
\hline 9 & 2 & 7 & 8 & 1 & 8 & 1490.5953 & 0 \\
\hline 15 & 2 & 13 & 14 & 2 & 12 & 1490.6545 & -4 \\
\hline 19 & 4 & 15 & 19 & 3 & 16 & 1490.6864 & -24 \\
\hline 23 & 2 & 21 & 22 & 3 & 20 & 1490.7022 & -5 \\
\hline 15 & 3 & 12 & 14 & 3 & 11 & 1490.7867 & 2 \\
\hline 10 & 2 & 9 & 9 & 1 & 8 & 1490.8141 & -2 \\
\hline 15 & 2 & 14 & 14 & 2 & 13 & 1490.8516 & -6 \\
\hline 15 & 7 & 8 & 14 & 7 & 7 & 1490.8516 & 1 \\
\hline 19 & 1 & 18 & 18 & 2 & 17 & 1490.8935 & -1 \\
\hline 20 & 4 & 16 & 20 & 3 & 17 & 1490.9024 & -18 \\
\hline 15 & 0 & 15 & 14 & 0 & 14 & 1491.0046 & -7 \\
\hline 20 & 4 & 17 & 20 & 3 & 18 & 1491.0588 & -15 \\
\hline 21 & 4 & 17 & 21 & 3 & 18 & 1491.1415 & -16 \\
\hline 6 & 3 & 4 & 5 & 2 & 3 & 1491.1852 & 0 \\
\hline 6 & 3 & 3 & 5 & 2 & 4 & 1491.1961 & 2 \\
\hline 30 & 2 & 29 & 30 & 1 & 30 & 1491.2316 & 12 \\
\hline 15 & 1 & 15 & 14 & 1 & 14 & 1491.2413 & -5 \\
\hline 14 & 1 & 14 & 13 & 0 & 13 & 1491.2537 & -3 \\
\hline 16 & 0 & 16 & 15 & 1 & 15 & 1491.2957 & -3 \\
\hline 21 & 4 & 18 & 21 & 3 & 19 & 1491.3486 & -10 \\
\hline 22 & 4 & 18 & 22 & 3 & 19 & 1491.3852 & -11 \\
\hline 14 & 8 & 7 & 15 & 7 & 8 & 1491.5641 & -1 \\
\hline 23 & 4 & 19 & 23 & 3 & 20 & 1491.6277 & -8 \\
\hline 22 & 4 & 19 & 22 & 3 & 20 & 1491.6545 & -10 \\
\hline 24 & 4 & 20 & 24 & 3 & 21 & 1491.8656 & -11 \\
\hline 9 & 7 & 3 & 10 & 6 & 4 & 1491.8788 & -7 \\
\hline 23 & 4 & 20 & 23 & 3 & 21 & 1491.9741 & -9 \\
\hline 25 & 4 & 21 & 25 & 3 & 22 & 1492.0972 & -13 \\
\hline 25 & 2 & 24 & 24 & 3 & 21 & 1492.1668 & 13 \\
\hline 16 & 1 & 15 & 15 & 1 & 14 & 1492.2454 & 2 \\
\hline 31 & 2 & 30 & 31 & 1 & 31 & 1492.2941 & 41 \\
\hline 24 & 4 & 21 & 24 & 3 & 22 & 1492.3065 & -8 \\
\hline 26 & 4 & 22 & 26 & 3 & 23 & 1492.3201 & -15 \\
\hline 11 & 2 & 10 & 10 & 1 & 9 & 1492.3787 & -1 \\
\hline 16 & 2 & 14 & 15 & 2 & 13 & 1492.4103 & 0 \\
\hline 10 & 2 & 8 & 9 & 1 & 9 & 1492.5086 & 0 \\
\hline 27 & 4 & 23 & 27 & 3 & 24 & 1492.5325 & -13 \\
\hline 16 & 3 & 13 & 15 & 3 & 12 & 1492.5852 & 2 \\
\hline 16 & 3 & 14 & 15 & 3 & 13 & 1492.6019 & 7 \\
\hline 16 & 4 & 12 & 15 & 4 & 11 & 1492.6093 & 3 \\
\hline 16 & 5 & 11 & 15 & 5 & 10 & 1492.6375 & 0 \\
\hline 25 & 4 & 22 & 25 & 3 & 23 & 1492.6506 & -14 \\
\hline 16 & 6 & 10 & 15 & 6 & 9 & 1492.6591 & 0 \\
\hline 16 & 2 & 15 & 15 & 2 & 14 & 1492.6692 & -1 \\
\hline 16 & 8 & 8 & 15 & 8 & 7 & 1492.7058 & -1 \\
\hline 16 & 7 & 9 & 15 & 7 & 8 & 1492.7058 & 13 \\
\hline 28 & 4 & 24 & 28 & 3 & 25 & 1492.7318 & -14 \\
\hline 16 & 9 & 7 & 15 & 9 & 6 & 1492.7463 & 11 \\
\hline 24 & 2 & 22 & 23 & 3 & 21 & 1492.7880 & -5 \\
\hline 13 & 8 & 6 & 14 & 7 & 7 & 1492.8458 & 3 \\
\hline 7 & 3 & 5 & 6 & 2 & 4 & 1492.8570 & 0 \\
\hline 16 & 0 & 16 & 15 & 0 & 15 & 1492.8660 & -3 \\
\hline 7 & 3 & 4 & 6 & 2 & 5 & 1492.8785 & 0 \\
\hline 29 & 4 & 25 & 29 & 3 & 26 & 1492.9166 & -16 \\
\hline 15 & 1 & 15 & 14 & 0 & 14 & 1492.9623 & -3 \\
\hline
\end{tabular}




\begin{tabular}{|c|c|c|c|}
\hline 19 & 4 & 15 & 18 \\
\hline 19 & 2 & 18 & 18 \\
\hline 18 & 1 & 18 & 17 \\
\hline 19 & 6 & 13 & 18 \\
\hline 19 & 7 & 12 & 18 \\
\hline 23 & 5 & 18 & 23 \\
\hline 23 & 5 & 19 & 23 \\
\hline 13 & 2 & 11 & 12 \\
\hline 19 & 9 & 10 & 18 \\
\hline 19 & 0 & 19 & 18 \\
\hline 15 & 2 & 14 & 14 \\
\hline 24 & 5 & 19 & 24 \\
\hline 24 & 5 & 20 & 24 \\
\hline 19 & 1 & 19 & 18 \\
\hline 25 & 5 & 20 & 25 \\
\hline 25 & 5 & 21 & 25 \\
\hline 27 & 2 & 25 & 26 \\
\hline 7 & 4 & 3 & 6 \\
\hline 26 & 5 & 21 & 26 \\
\hline 20 & 1 & 19 & 19 \\
\hline 26 & 5 & 22 & 26 \\
\hline 23 & 1 & 22 & 22 \\
\hline 20 & 0 & 20 & 19 \\
\hline 11 & 3 & 9 & 10 \\
\hline 27 & 5 & 22 & 27 \\
\hline 11 & 3 & 8 & 10 \\
\hline 27 & 5 & 23 & 27 \\
\hline 20 & 3 & 17 & 19 \\
\hline 20 & 3 & 18 & 19 \\
\hline 20 & 4 & 16 & 19 \\
\hline 19 & 1 & 19 & 18 \\
\hline 20 & 5 & 15 & 19 \\
\hline 28 & 5 & 23 & 28 \\
\hline 20 & 2 & 19 & 19 \\
\hline 20 & 6 & 14 & 19 \\
\hline 28 & 5 & 24 & 28 \\
\hline 16 & 2 & 15 & 15 \\
\hline 20 & 7 & 13 & 19 \\
\hline 20 & 8 & 12 & 19 \\
\hline 29 & 5 & 24 & 29 \\
\hline 29 & 5 & 25 & 29 \\
\hline 20 & 9 & 11 & 19 \\
\hline 20 & 0 & 20 & 19 \\
\hline 14 & 2 & 12 & 13 \\
\hline 30 & 5 & 25 & 30 \\
\hline 6 & 6 & 0 & 6 \\
\hline 20 & 1 & 20 & 19 \\
\hline 30 & 5 & 26 & 30 \\
\hline 8 & 4 & 4 & 7 \\
\hline 8 & 6 & 2 & 8 \\
\hline 9 & 6 & 3 & 9 \\
\hline 21 & 1 & 20 & 20 \\
\hline 12 & 3 & 10 & 11 \\
\hline 10 & 6 & 4 & 10 \\
\hline 12 & 3 & 9 & 11 \\
\hline 11 & 6 & 5 & 11 \\
\hline 21 & 0 & 21 & 20 \\
\hline 24 & 1 & 23 & 23 \\
\hline 21 & 3 & 18 & 20 \\
\hline 17 & 2 & 16 & 16 \\
\hline 21 & 3 & 19 & 20 \\
\hline 12 & 6 & 6 & 12 \\
\hline 20 & 1 & 20 & 19 \\
\hline 21 & 4 & 17 & 20 \\
\hline 21 & 5 & 16 & 20 \\
\hline 21 & 2 & 20 & 20 \\
\hline 21 & 6 & 15 & 20 \\
\hline 13 & 6 & 7 & 13 \\
\hline 21 & 7 & 14 & 20 \\
\hline 5 & 5 & 0 & 4 \\
\hline 14 & 6 & 8 & 14 \\
\hline 21 & 8 & 13 & 20 \\
\hline 21 & 0 & 21 & 20 \\
\hline 15 & 6 & 9 & 15 \\
\hline 9 & 4 & 5 & 8 \\
\hline 15 & 2 & 13 & 14 \\
\hline 16 & 6 & 10 & 16 \\
\hline
\end{tabular}

\begin{tabular}{|c|c|c|c|}
\hline 4 & 14 & 1498.1977 & -10 \\
\hline 2 & 17 & 1498.2126 & 4 \\
\hline 0 & 17 & 1498.2283 & -4 \\
\hline 6 & 12 & 1498.2539 & 3 \\
\hline 7 & 11 & 1498.3565 & 5 \\
\hline 4 & 19 & 1498.4280 & 13 \\
\hline 4 & 20 & 1498.4424 & 5 \\
\hline 1 & 12 & 1498.5073 & -2 \\
\hline 9 & 9 & 1498.5774 & 7 \\
\hline 0 & 18 & 1498.5905 & 2 \\
\hline 1 & 13 & 1498.6333 & 0 \\
\hline 4 & 20 & 1498.7497 & -3 \\
\hline 4 & 21 & 1498.7719 & 8 \\
\hline 1 & 18 & 1498.8835 & -8 \\
\hline 4 & 21 & 1499.0781 & 0 \\
\hline 4 & 22 & 1499.1078 & 7 \\
\hline 3 & 24 & 1499.2172 & 3 \\
\hline 3 & 4 & 1499.2378 & -3 \\
\hline 4 & 22 & 1499.4099 & 0 \\
\hline 1 & 18 & 1499.4099 & 4 \\
\hline 4 & 23 & 1499.4504 & 11 \\
\hline 2 & 21 & 1499.4719 & -4 \\
\hline 1 & 19 & 1499.5186 & -2 \\
\hline 2 & 8 & 1499.6307 & 1 \\
\hline 4 & 23 & 1499.7445 & 4 \\
\hline 2 & 9 & 1499.7815 & 1 \\
\hline 4 & 24 & 1499.7974 & 5 \\
\hline 3 & 16 & 1499.8681 & 6 \\
\hline 3 & 17 & 1499.9338 & 5 \\
\hline 4 & 15 & 1500.0244 & -7 \\
\hline 0 & 18 & 1500.0349 & -3 \\
\hline 5 & 14 & 1500.0552 & 7 \\
\hline 4 & 24 & 1500.0792 & -1 \\
\hline 2 & 18 & 1500.0901 & -2 \\
\hline 6 & 13 & 1500.1428 & 5 \\
\hline 4 & 25 & 1500.1490 & -3 \\
\hline 1 & 14 & 1500.1991 & 1 \\
\hline 7 & 12 & 1500.2661 & 5 \\
\hline 8 & 11 & 1500.3624 & 12 \\
\hline 4 & 25 & 1500.4135 & -6 \\
\hline 4 & 26 & 1500.5048 & -9 \\
\hline 9 & 10 & 1500.5458 & 5 \\
\hline 0 & 19 & 1500.5458 & 8 \\
\hline 1 & 13 & 1500.5989 & -2 \\
\hline 4 & 26 & 1500.7436 & -28 \\
\hline 5 & 1 & 1500.8027 & -5 \\
\hline 1 & 19 & 1500.8421 & -5 \\
\hline 4 & 27 & 1500.8637 & -16 \\
\hline 3 & 5 & 1500.9356 & -2 \\
\hline 5 & 3 & 1501.0767 & -4 \\
\hline 5 & 4 & 1501.2395 & -2 \\
\hline 1 & 19 & 1501.2395 & 9 \\
\hline 2 & 9 & 1501.3349 & -2 \\
\hline 5 & 5 & 1501.4186 & -4 \\
\hline 2 & 10 & 1501.5521 & -2 \\
\hline 5 & 6 & 1501.6131 & -13 \\
\hline 1 & 20 & 1501.6131 & 11 \\
\hline 2 & 22 & 1501.6763 & 1 \\
\hline 3 & 17 & 1501.7054 & 2 \\
\hline 1 & 15 & 1501.7673 & 1 \\
\hline 3 & 18 & 1501.7939 & -1 \\
\hline 5 & 7 & 1501.8256 & 0 \\
\hline 0 & 19 & 1501.8684 & -3 \\
\hline 4 & 16 & 1501.8836 & -2 \\
\hline 5 & 15 & 1501.9362 & 1 \\
\hline 2 & 19 & 1501.9837 & -1 \\
\hline 6 & 14 & 1502.0417 & -3 \\
\hline 5 & 8 & 1502.0522 & 2 \\
\hline 7 & 13 & 1502.1872 & 5 \\
\hline 4 & 1 & 1502.2246 & -1 \\
\hline 5 & 9 & 1502.2933 & 2 \\
\hline 8 & 12 & 1502.3038 & 9 \\
\hline 0 & 20 & 1502.5232 & 10 \\
\hline 5 & 10 & 1502.5487 & 3 \\
\hline 3 & 6 & 1502.6483 & -2 \\
\hline 1 & 14 & 1502.7396 & -1 \\
\hline 5 & 11 & 1502.8176 & \\
\hline
\end{tabular}

22
13
2
1
1
1
1
22
2

2

19

19

21

22

2

2

22
20

22

2

1

2

2

14

2

19

16

23

14

24
23

23

2

22
23

23
23

25

23

23

3

2

26
26

26
23

15

20
27

27
23

23

24
23

24

28

15
17

17
7

24
29

29

8
2
2
2
3
30
10
24 $\begin{array}{llllllrl}2 & 20 & 21 & 2 & 19 & 1503.0181 & -11 & 4 \\ 3 & 11 & 12 & 2 & 10 & 1503.0397 & 2 & 4 \\ 1 & 21 & 21 & 1 & 20 & 1503.0844 & -1 & 4\end{array}$ $\begin{array}{rrrrrrrr}1 & 21 & 21 & 1 & 20 & 1503.0844 & -1 & 4\end{array}$ $\begin{array}{rrrrrrrr}3 & 10 & 12 & 2 & 11 & 1503.3403 & -19 & 10\end{array}$ $\begin{array}{llllllll}2 & 17 & 17 & 1 & 16 & 1503.3403 & 15 & 10\end{array}$ $\begin{array}{llllllrr}6 & 12 & 18 & 5 & 13 & 1503.3936 & 3 & 4\end{array}$ $\begin{array}{llllllll}3 & 19 & 21 & 3 & 18 & 1503.5479 & 4 & 4\end{array}$ $\begin{array}{llllllll}2 & 27 & 28 & 3 & 26 & 1503.6415 & -8 & 4\end{array}$ $\begin{array}{llllllll}3 & 20 & 21 & 3 & 19 & 1503.6655 & 4 & 4\end{array}$ $\begin{array}{llllllll}6 & 13 & 19 & 5 & 14 & 1503.6999 & 6 & 4\end{array}$ $\begin{array}{llllllll}0 & 22 & 21 & 1 & 21 & 1503.7173 & -6 & 4\end{array}$ $\begin{array}{llllllll}1 & 21 & 20 & 0 & 20 & 1503.7290 & -4 & 4 \\ 4 & 18 & 21 & 4 & 17 & 1503.7563 & -6 & 4\end{array}$ $\begin{array}{llllllll}4 & 18 & 21 & 4 & 17 & 1503.7563 & -6 & 4\end{array}$ $\begin{array}{lll}5 & 17 & 21\end{array}$ $\begin{array}{rrr}5 & 1 & 5 \\ 6 & 16 & 21\end{array}$ 516 $\begin{array}{lll}6 & 16 & 21 \\ 6 & 14 & 20\end{array}$ $\begin{array}{lll}7 & 15 & 21\end{array}$ $\begin{array}{lll}8 & 14 & 21 \\ 6 & 15 & 21\end{array}$ $\begin{array}{lrr}6 & 15 & 21 \\ 4 & 6 & 9\end{array}$ $\begin{array}{lll}9 & 13 & 21\end{array}$ $\begin{array}{lll}0 & 22 & 21 \\ 6 & 16 & 22\end{array}$ $3 \quad 12 \quad 13$ $\begin{array}{lll}2 & 21 & 22\end{array}$ $\begin{array}{lll}1 & 22 & 21\end{array}$ $\begin{array}{lll}2 & 18 & 18\end{array}$ $\begin{array}{lll}2 & 14 & 15 \\ 1 & 22 & 22\end{array}$ $\begin{array}{lll}1 & 22 & 22 \\ 6 & 17 & 23\end{array}$ $\begin{array}{lll}3 & 11 & 13\end{array}$ $\begin{array}{lll}6 & 18 & 24\end{array}$ $\begin{array}{lll}3 & 20 & 22\end{array}$ $\begin{array}{rrr}3 & 21 & 22\end{array}$ $\begin{array}{lll}1 & 22 & 21 \\ 4 & 19 & 22\end{array}$ $\begin{array}{lll}4 & 19 & 22 \\ 4 & 20 & 22\end{array}$ $\begin{array}{lll}5 & 18 & 22\end{array}$ $\begin{array}{lll}6 & 19 & 25\end{array}$ $\begin{array}{lll}2 & 22 & 22 \\ 0 & 23 & 22\end{array}$ $\begin{array}{lll}6 & 17 & 22\end{array}$ $\begin{array}{lll}2 & 28 & 29\end{array}$ $\begin{array}{lll}7 & 16 & 22\end{array}$ $\begin{array}{rrr}6 & 20 & 26 \\ 4 & 7 & 10\end{array}$ $\begin{array}{lll}1 & 25 & 25\end{array}$ $\begin{array}{lll}8 & 15 & 22\end{array}$ $\begin{array}{lll}3 & 13 & 14 \\ 2 & 19 & 19\end{array}$ $\begin{array}{lll}2 & 19 & 19 \\ 6 & 21 & 27\end{array}$ $\begin{array}{lll}9 & 14 & 22\end{array}$ $\begin{array}{rrr}1503.7604 & -19 & 4 \\ 1503.8283 & 9 & 4\end{array}$ $\begin{array}{lll}1503.8959 & -1 & 4\end{array}$ $1503.9518 \quad-3 \quad 4$ $\begin{array}{rrr}1504.0170 & 6 & 4\end{array}$ $1504.1192 \quad 6 \quad 4$ $1504.2551 \quad 14 \quad 4$ $\begin{array}{rrr}1504.3448 & 7 & 4\end{array}$ $\begin{array}{rrr}1504.3750 & -7 & 4 \\ 1504.5146 & 3 & 4\end{array}$ $1504.5207 \quad-6 \quad 4$ $\begin{array}{rrr}1504.6823 & 7 & 4\end{array}$ $\begin{array}{lll}1504.7412 & -1 & 4\end{array}$ $1504.8001 \quad-12 \quad 4$ $1504.8149 \quad 10 \quad 4$ $\begin{array}{rrr}1504.8149 & 10 & 4 \\ 1504.9150 & 0 & 4\end{array}$ $1504.9305 \quad-5 \quad 4$ $1504.9487 \quad 7 \quad 4$ $1505.0290 \quad 7 \quad 4$ $\begin{array}{lll}1505.1515 & -3 & 4\end{array}$ $1505.3841 \quad 6 \quad 4$ $1505.3921 \quad-11 \quad 4$ $\begin{array}{rrr}1505.5470 & 3 & 4\end{array}$ $1505.5838 \quad-1 \quad 4$ $\begin{array}{lll}1505.6169 & -5 & 4\end{array}$ $1505.6393 \quad-11 \quad 4$ $1505.6463 \quad-19 \quad 4$ $\begin{array}{lll}1505.7286 & 9 & 4\end{array}$ $1505.7476 \quad 11 \quad 4$ $1505.8172 \quad 9 \quad 4$ $\begin{array}{lll}1505.8350 & -3 & 4\end{array}$ $1505.8725 \quad 5 \quad 4$ $\begin{array}{rrr}1505.8954 & -1 & 4 \\ 1506.0607 & 1 & 4\end{array}$ $1506.1156 \quad-12 \quad 10$ $1506.1156-12 \quad 10$ $1506.1381 \quad 5 \quad 4$ $1506.2105 \quad-14 \quad 4$ $\begin{array}{rrr}1506.4382 & -1 & 4\end{array}$ $1506.4965 \quad-4 \quad 10$ $1506.4965 \quad 30 \quad 10$ $1506.5119 \quad-10 \quad 4$ $\begin{array}{lll}1506.5408 & -7 & 4\end{array}$ $\begin{array}{llllllll}2 & 22 & 23 & 2 & 21 & 1506.5883 & -6 & 4 \\ 1 & 23 & 22 & 1 & 22 & 1506.8255 & -8 & 4\end{array}$ $\begin{array}{rrrrrrrr}1 & 23 & 23 & 1 & 22 & 1506.8333 & 0 & 4 \\ 6 & 22 & 28 & 5 & 23 & 1506.8776 & 16 & 4\end{array}$ $\begin{array}{llllllll}3 & 12 & 14 & 2 & 13 & 1506.9817 & -1 & 4\end{array}$ $\begin{array}{llllllll}2 & 15 & 16 & 1 & 16 & 1507.1745 & -4 & 4\end{array}$ $\begin{array}{rrrrrrrr}7 & 0 & 7 & 6 & 1 & 1507.2122 & -7 & 4\end{array}$ $\begin{array}{llllllll}3 & 21 & 23 & 3 & 20 & 1507.2408 & -2 & 4\end{array}$ $\begin{array}{rrrrrrrr}6 & 23 & 29 & 5 & 24 & 1507.2634 & -1 & 4 \\ 6 & 24 & 29 & 5 & 25 & 1507.2674 & 4 & 4\end{array}$ $\begin{array}{rrrrrrrr}5 & 3 & 7 & 4 & 4 & 1507.2877 & -2 & 4\end{array}$ $\begin{array}{llllllll}7 & 1 & 8 & 6 & 2 & 1507.3697 & -3 & 4\end{array}$ $\begin{array}{llllllll}3 & 22 & 23 & 3 & 21 & 1507.4389 & 4 & 4\end{array}$ $\begin{array}{rrrrrrrr}1 & 23 & 22 & 0 & 22 & 1507.5320 & -5 & 4 \\ 7 & 2 & 9 & 6 & 3 & 1507.5445 & -6 & 4\end{array}$ $\begin{array}{llllllll}5 & 19 & 23 & 5 & 18 & 1507.6380 & 14 & 4\end{array}$ $\begin{array}{llllllll}6 & 24 & 30 & 5 & 25 & 1507.6553 & -1 & 4\end{array}$ $\begin{array}{llllllll}6 & 25 & 30 & 5 & 26 & 1507.6610 & 7 & 4\end{array}$ $\begin{array}{rrrrrrrrr}10 & 7 & 3 & 10 & 6 & 4 & 1507.7369 & -3 & 4 \\ 24 & 2 & 23 & 23 & 2 & 22 & 1507.7563 & 9 & 4\end{array}$ 


\begin{tabular}{|c|c|c|c|c|c|c|c|c|}
\hline 24 & 6 & 18 & 23 & 6 & 17 & 1507.8017 & 6 & 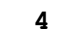 \\
\hline 12 & 4 & 9 & 11 & 3 & 8 & 1507.8668 & 2 & 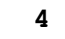 \\
\hline 12 & 4 & 8 & 11 & 3 & 9 & 1507.8711 & -3 & 4 \\
\hline 11 & 7 & 4 & 11 & 6 & 5 & 1507.9449 & -2 & 4 \\
\hline 24 & 0 & 24 & 23 & 1 & 23 & 1507.9633 & -3 & \\
\hline 24 & 7 & 17 & 23 & 7 & 16 & 1508.0121 & 0 & \\
\hline 21 & 2 & 20 & 20 & 1 & 19 & 1508.0862 & 2 & \\
\hline 16 & 3 & 14 & 15 & 2 & 13 & 1508.1285 & 1 & \\
\hline 12 & 7 & 5 & 12 & 6 & 6 & 1508.1645 & 0 & \\
\hline 31 & 2 & 29 & 30 & 3 & 28 & 1508.1735 & -14 & 10 \\
\hline 24 & 8 & 16 & 23 & 8 & 15 & 1508.1735 & -22 & 10 \\
\hline 13 & 7 & 6 & 13 & 6 & 7 & 1508.3643 & 11 & \\
\hline 25 & 2 & 23 & 24 & 2 & 22 & 1508.3823 & -6 & \\
\hline 27 & 1 & 26 & 26 & 2 & 25 & 1508.3984 & 8 & \\
\hline 24 & 9 & 15 & 23 & 9 & 14 & 1508.5181 & -18 & \\
\hline 24 & 0 & 24 & 23 & 0 & 23 & 1508.5823 & 7 & \\
\hline 14 & 7 & 7 & 14 & 6 & 8 & 1508.7234 & -8 & \\
\hline 25 & 1 & 24 & 24 & 1 & 23 & 1508.7341 & 6 & \\
\hline 16 & 3 & 13 & 15 & 2 & 14 & 1508.8331 & 1 & \\
\hline 24 & 1 & 24 & 23 & 1 & 23 & 1508.8561 & 1 & \\
\hline 15 & 7 & 8 & 15 & 6 & 9 & 1508.9800 & -1 & \\
\hline 9 & 5 & 4 & 8 & 4 & 5 & 1509.0078 & 1 & \\
\hline 25 & 3 & 22 & 24 & 3 & 21 & 1509.0893 & -6 & \\
\hline 16 & 7 & 9 & 16 & 6 & 10 & 1509.2641 & 2 & \\
\hline 25 & 3 & 23 & 24 & 3 & 22 & 1509.3405 & 0 & \\
\hline 25 & 4 & 21 & 24 & 4 & 20 & 1509.4326 & -1 & \\
\hline 25 & 4 & 22 & 24 & 4 & 21 & 1509.4468 & -12 & \\
\hline 24 & 1 & 24 & 23 & 0 & 23 & 1509.4733 & -7 & 10 \\
\hline 18 & 2 & 16 & 17 & 1 & 17 & 1509.4733 & 3 & 10 \\
\hline 25 & 5 & 20 & 24 & 5 & 19 & 1509.5542 & 11 & \\
\hline 17 & 7 & 10 & 17 & 6 & 11 & 1509.5648 & 2 & \\
\hline 13 & 4 & 10 & 12 & 3 & 9 & 1509.6310 & 2 & \\
\hline 13 & 4 & 9 & 12 & 3 & 10 & 1509.6389 & 1 & \\
\hline 22 & 2 & 21 & 21 & 1 & 20 & 1509.6844 & 6 & \\
\hline 25 & 2 & 24 & 24 & 2 & 23 & 1509.7119 & 23 & \\
\hline 25 & 6 & 19 & 24 & 6 & 18 & 1509.7392 & 5 & \\
\hline 17 & 3 & 15 & 16 & 2 & 14 & 1509.8097 & 0 & \\
\hline 18 & 7 & 11 & 18 & 6 & 12 & 1509.8799 & 5 & \\
\hline 25 & 7 & 18 & 24 & 7 & 17 & 1509.9730 & 6 & \\
\hline 25 & 0 & 25 & 24 & 1 & 24 & 1510.1016 & -4 & \\
\hline 25 & 8 & 17 & 24 & 8 & 16 & 1510.1367 & -53 & \\
\hline 26 & 2 & 24 & 25 & 2 & 23 & 1510.1844 & 2 & \\
\hline 6 & 6 & 0 & 5 & 5 & 1 & 1510.2061 & -1 & \\
\hline 32 & 2 & 30 & 31 & 3 & 29 & 1510.4796 & -3 & \\
\hline 25 & 9 & 16 & 24 & 9 & 15 & 1510.5323 & -20 & \\
\hline 20 & 7 & 13 & 20 & 6 & 14 & 1510.5479 & 5 & \\
\hline 25 & 0 & 25 & 24 & 0 & 24 & 1510.6415 & 7 & \\
\hline 26 & 1 & 25 & 25 & 1 & 24 & 1510.6574 & 12 & \\
\hline 28 & 1 & 27 & 27 & 2 & 26 & 1510.6756 & 8 & \\
\hline 17 & 3 & 14 & 16 & 2 & 15 & 1510.7063 & -1 & \\
\hline 10 & 5 & 5 & 9 & 4 & 6 & 1510.7429 & -1 & \\
\hline 21 & 7 & 14 & 21 & 6 & 15 & 1510.8999 & 11 & \\
\hline 26 & 3 & 23 & 25 & 3 & 22 & 1510.9399 & 14 & \\
\hline 26 & 3 & 24 & 25 & 3 & 23 & 1511.2538 & 13 & \\
\hline 22 & 7 & 15 & 22 & 6 & 16 & 1511.2616 & 8 & \\
\hline 23 & 2 & 22 & 22 & 1 & 21 & 1511.2925 & 5 & \\
\hline 26 & 4 & 22 & 25 & 4 & 21 & 1511.3399 & 4 & \\
\hline 26 & 4 & 23 & 25 & 4 & 22 & 1511.3598 & -6 & \\
\hline 14 & 4 & 11 & 13 & 3 & 10 & 1511.4058 & 2 & \\
\hline 14 & 4 & 10 & 13 & 3 & 11 & 1511.4185 & 0 & \\
\hline 25 & 1 & 25 & 24 & 0 & 24 & 1511.4410 & -2 & \\
\hline 18 & 3 & 16 & 17 & 2 & 15 & 1511.4804 & -1 & \\
\hline 23 & 7 & 16 & 23 & 6 & 17 & 1511.6337 & 8 & \\
\hline 26 & 2 & 25 & 25 & 2 & 24 & 1511.6798 & 8 & \\
\hline 26 & 6 & 20 & 25 & 6 & 19 & 1511.6860 & 16 & \\
\hline 19 & 2 & 17 & 18 & 1 & 18 & 1511.8263 & -6 & \\
\hline 7 & 6 & 1 & 6 & 5 & 2 & 1511.9006 & -6 & \\
\hline 26 & 7 & 19 & 25 & 7 & 18 & 1511.9407 & -3 & \\
\hline 27 & 2 & 25 & 26 & 2 & 24 & 1511.9932 & -4 & \\
\hline 24 & 7 & 17 & 24 & 6 & 18 & 1512.0148 & 6 & \\
\hline 26 & 0 & 26 & 25 & 1 & 25 & 1512.2497 & -2 & \\
\hline 25 & 7 & 18 & 25 & 6 & 19 & 1512.4051 & 9 & \\
\hline 11 & 5 & 6 & 10 & 4 & 7 & 1512.4930 & -1 & \\
\hline 18 & 3 & 15 & 17 & 2 & 16 & 1512.6029 & -1 & \\
\hline 26 & 0 & 26 & 25 & 0 & 25 & 1512.7187 & 7 & \\
\hline 27 & 3 & 24 & 26 & 3 & 23 & 1512.7859 & 1 & \\
\hline 26 & 7 & 19 & 26 & 6 & 20 & 1512.8030 & 8 & \\
\hline
\end{tabular}




\begin{tabular}{|c|c|c|c|c|c|c|c|}
\hline 18 & 10 & 8 & 18 & 9 & 9 & 1529.3401 & 14 \\
\hline 24 & 4 & 21 & 23 & 3 & 20 & 1529.5321 & -13 \\
\hline 10 & 8 & 2 & 9 & 7 & 3 & 1529.6818 & -8 \\
\hline 17 & 6 & 11 & 16 & 5 & 12 & 1529.7222 & 5 \\
\hline 34 & 0 & 34 & 33 & 1 & 33 & 1529.7310 & 5 \\
\hline 19 & 10 & 9 & 19 & 9 & 10 & 1529.7760 & 12 \\
\hline 24 & 4 & 20 & 23 & 3 & 21 & 1529.8857 & -15 \\
\hline 34 & 1 & 34 & 33 & 0 & 33 & 1530.1281 & -1 \\
\hline 34 & 2 & 33 & 33 & 1 & 32 & 1530.2070 & 19 \\
\hline 20 & 10 & 10 & 20 & 9 & 11 & 1530.2230 & 9 \\
\hline 30 & 3 & 28 & 29 & 2 & 27 & 1530.5878 & 11 \\
\hline 14 & 7 & 7 & 13 & 6 & 8 & 1530.6486 & -6 \\
\hline 21 & 10 & 11 & 21 & 9 & 12 & 1530.6801 & 5 \\
\hline 21 & 5 & 17 & 20 & 4 & 16 & 1530.6895 & 6 \\
\hline 21 & 5 & 16 & 20 & 4 & 17 & 1530.6941 & 1 \\
\hline 27 & 3 & 24 & 26 & 2 & 25 & 1530.9333 & 0 \\
\hline 22 & 10 & 12 & 22 & 9 & 13 & 1531.1462 & -4 \\
\hline 25 & 4 & 22 & 24 & 3 & 21 & 1531.3391 & -16 \\
\hline 11 & 8 & 3 & 10 & 7 & 4 & 1531.4778 & -4 \\
\hline 18 & 6 & 12 & 17 & 5 & 13 & 1531.5795 & 3 \\
\hline 23 & 10 & 13 & 23 & 9 & 14 & 1531.6210 & -10 \\
\hline 25 & 4 & 21 & 24 & 3 & 22 & 1531.7901 & -13 \\
\hline 35 & 0 & 35 & 34 & 1 & 34 & 1531.9489 & -4 \\
\hline 24 & 10 & 14 & 24 & 9 & 15 & 1532.1031 & -19 \\
\hline 31 & 3 & 29 & 30 & 2 & 28 & 1532.1223 & 5 \\
\hline 35 & 1 & 35 & 34 & 0 & 34 & 1532.2927 & 0 \\
\hline 15 & 7 & 8 & 14 & 6 & 9 & 1532.4688 & -1 \\
\hline 22 & 5 & 18 & 21 & 4 & 17 & 1532.5633 & 4 \\
\hline 22 & 5 & 17 & 21 & 4 & 18 & 1532.5706 & 2 \\
\hline 11 & 11 & 0 & 11 & 10 & 1 & 1532.7577 & -17 \\
\hline 27 & 2 & 25 & 26 & 1 & 26 & 1532.7863 & -3 \\
\hline 26 & 4 & 23 & 25 & 3 & 22 & 1533.1387 & -10 \\
\hline 28 & 3 & 25 & 27 & 2 & 26 & 1533.1387 & -21 \\
\hline 12 & 11 & 1 & 12 & 10 & 2 & 1533.1589 & -17 \\
\hline 12 & 8 & 4 & 11 & 7 & 5 & 1533.2911 & -3 \\
\hline 19 & 6 & 13 & 18 & 5 & 14 & 1533.4484 & 4 \\
\hline 13 & 11 & 2 & 13 & 10 & 3 & 1533.5813 & 0 \\
\hline 26 & 4 & 22 & 25 & 3 & 23 & 1533.7060 & -15 \\
\hline 14 & 11 & 3 & 14 & 10 & 4 & 1534.0197 & 2 \\
\hline 9 & 9 & 0 & 8 & 8 & 1 & 1534.1000 & -17 \\
\hline 36 & 0 & 36 & 35 & 1 & 35 & 1534.1737 & -12 \\
\hline 16 & 7 & 9 & 15 & 6 & 10 & 1534.3161 & 0 \\
\hline 23 & 5 & 19 & 22 & 4 & 18 & 1534.4443 & 8 \\
\hline 23 & 5 & 18 & 22 & 4 & 19 & 1534.4546 & 3 \\
\hline 15 & 11 & 4 & 15 & 10 & 5 & 1534.4751 & 17 \\
\hline 27 & 4 & 24 & 26 & 3 & 23 & 1534.9264 & -19 \\
\hline 16 & 11 & 5 & 16 & 10 & 6 & 1534.9430 & 15 \\
\hline 13 & 8 & 5 & 12 & 7 & 6 & 1535.1212 & 0 \\
\hline 20 & 6 & 14 & 19 & 5 & 15 & 1535.3277 & 3 \\
\hline 29 & 3 & 26 & 28 & 2 & 27 & 1535.3888 & -8 \\
\hline 17 & 11 & 6 & 17 & 10 & 7 & 1535.4236 & 13 \\
\hline 27 & 4 & 23 & 26 & 3 & 24 & 1535.6349 & -9 \\
\hline 28 & 2 & 26 & 27 & 1 & 27 & 1535.6783 & 2 \\
\hline 10 & 9 & 1 & 9 & 8 & 2 & 1535.9053 & -12 \\
\hline 18 & 11 & 7 & 18 & 10 & 8 & 1535.9154 & 10 \\
\hline 17 & 7 & 10 & 16 & 6 & 11 & 1536.1800 & 3 \\
\hline 24 & 5 & 20 & 23 & 4 & 19 & 1536.3302 & 4 \\
\hline 24 & 5 & 19 & 23 & 4 & 20 & 1536.3455 & 4 \\
\hline 19 & 11 & 8 & 19 & 10 & 9 & 1536.4177 & 12 \\
\hline 37 & 1 & 37 & 36 & 0 & 36 & 1536.6615 & -5 \\
\hline 28 & 4 & 25 & 27 & 3 & 24 & 1536.7024 & -16 \\
\hline 20 & 11 & 9 & 20 & 10 & 10 & 1536.9276 & 0 \\
\hline 14 & 8 & 6 & 13 & 7 & 7 & 1536.9671 & 1 \\
\hline 21 & 6 & 15 & 20 & 5 & 16 & 1537.2171 & 3 \\
\hline 21 & 11 & 10 & 21 & 10 & 11 & 1537.4464 & -2 \\
\hline 28 & 4 & 24 & 27 & 3 & 25 & 1537.5751 & -17 \\
\hline 11 & 9 & 2 & 10 & 8 & 3 & 1537.7302 & -9 \\
\hline 22 & 11 & 11 & 22 & 10 & 12 & 1537.9709 & -17 \\
\hline 18 & 7 & 11 & 17 & 6 & 12 & 1538.0572 & 3 \\
\hline 25 & 5 & 21 & 24 & 4 & 20 & 1538.2213 & 6 \\
\hline 25 & 5 & 20 & 24 & 4 & 21 & 1538.2429 & 9 \\
\hline 29 & 4 & 26 & 28 & 3 & 25 & 1538.4627 & -21 \\
\hline 15 & 8 & 7 & 14 & 7 & 8 & 1538.8285 & 6 \\
\hline 22 & 6 & 16 & 21 & 5 & 17 & 1539.1158 & 3 \\
\hline 29 & 4 & 25 & 28 & 3 & 26 & 1539.5296 & -15 \\
\hline 12 & 9 & 3 & 11 & 8 & 4 & 1539.5745 & -2 \\
\hline 19 & 7 & 12 & 18 & 6 & 13 & 1539.9470 & \\
\hline
\end{tabular}




$\begin{array}{rrrrrrrrr}26 & 5 & 22 & 25 & 4 & 21 & 1540.1158 & 7 & 4 \\ 26 & 5 & 21 & 25 & 4 & 22 & 1540.1454 & 10 & 4 \\ 30 & 4 & 27 & 29 & 3 & 26 & 1540.2065 & -22 & 4 \\ 16 & 8 & 8 & 15 & 7 & 9 & 1540.7036 & 6 & 4 \\ 23 & 6 & 17 & 22 & 5 & 18 & 1541.0229 & 1 & 4 \\ 13 & 9 & 4 & 12 & 8 & 5 & 1541.4361 & -1 & 4 \\ 30 & 4 & 26 & 29 & 3 & 27 & 1541.4976 & -19 & 4 \\ 20 & 7 & 13 & 19 & 6 & 14 & 1541.8485 & 6 & 4 \\ 27 & 5 & 23 & 26 & 4 & 22 & 1542.0121 & 3 & 4 \\ 10 & 10 & 0 & 9 & 9 & 1 & 1542.0425 & -20 & 4 \\ 27 & 5 & 22 & 26 & 4 & 23 & 1542.0524 & 9 & 4 \\ 17 & 8 & 9 & 16 & 7 & 10 & 1542.5923 & 7 & 4 \\ 24 & 6 & 18 & 23 & 5 & 19 & 1542.9386 & 5 & 4 \\ 14 & 9 & 5 & 13 & 8 & 6 & 1543.3150 & 2 & 4 \\ 21 & 7 & 14 & 20 & 6 & 15 & 1543.7604 & 5 & 4 \\ 11 & 10 & 1 & 10 & 9 & 2 & 1543.9114 & -19 & 4 \\ 28 & 5 & 23 & 27 & 4 & 24 & 1543.9627 & 1 & 4 \\ 18 & 8 & 10 & 17 & 7 & 11 & 1544.4936 & 8 & 4 \\ 25 & 6 & 19 & 24 & 5 & 20 & 1544.8611 & 4 & 4 \\ 15 & 9 & 6 & 14 & 8 & 7 & 1545.2098 & 5 & 4 \\ 22 & 7 & 15 & 21 & 6 & 16 & 1545.6826 & 6 & 4 \\ 12 & 10 & 2 & 11 & 9 & 3 & 1545.8018 & -7 & 4 \\ 29 & 5 & 24 & 28 & 4 & 25 & 1545.8757 & -11 & 4 \\ 19 & 8 & 11 & 18 & 7 & 12 & 1546.4061 & 6 & 4 \\ 26 & 6 & 20 & 25 & 5 & 21 & 1546.7903 & 4 & 4 \\ 16 & 9 & 7 & 15 & 8 & 8 & 1547.1192 & 3 & 4 \\ 23 & 7 & 16 & 22 & 6 & 17 & 1547.6141 & 8 & 4 \\ 30 & 5 & 26 & 29 & 4 & 25 & 1547.6980 & -27 & 4 \\ 13 & 10 & 3 & 12 & 9 & 4 & 1547.7106 & -2 & 4 \\ 30 & 5 & 25 & 29 & 4 & 26 & 1547.7897 & -36 & 4 \\ 20 & 8 & 12 & 19 & 7 & 13 & 1548.3292 & 3 & 4 \\ 27 & 6 & 21 & 26 & 5 & 22 & 1548.7250 & 0 & 4 \\ 17 & 9 & 8 & 16 & 8 & 9 & 1549.0434 & 9 & 4 \\ 24 & 7 & 17 & 23 & 6 & 18 & 1549.5537 & 5 & 4 \\ 14 & 10 & 4 & 13 & 9 & 5 & 1549.6377 & 9 & 4\end{array}$

$\begin{array}{rrrr}12 & 7 & 5 & 13 \\ 18 & 5 & 13 & 19 \\ 14 & 6 & 8 & 15 \\ 17 & 5 & 12 & 18 \\ 10 & 7 & 3 & 11 \\ 13 & 6 & 7 & 14 \\ 9 & 7 & 2 & 10 \\ 8 & 7 & 1 & 9 \\ 15 & 5 & 10 & 16 \\ 11 & 6 & 5 & 12 \\ 7 & 7 & 0 & 8 \\ 14 & 5 & 9 & 15 \\ 13 & 5 & 8 & 14 \\ 7 & 6 & 1 & 8 \\ 14 & 4 & 10 & 15 \\ 10 & 5 & 5 & 11 \\ 6 & 6 & 0 & 7 \\ 9 & 5 & 4 & 10 \\ 12 & 4 & 8 & 13 \\ 26 & 0 & 26 & 27 \\ 8 & 5 & 3 & 9 \\ 18 & 2 & 17 & 19 \\ 7 & 5 & 2 & 8 \\ 25 & 1 & 25 & 26 \\ 25 & 0 & 25 & 26 \\ 25 & 1 & 25 & 26 \\ 14 & 3 & 11 & 15 \\ 10 & 4 & 6 & 11 \\ 6 & 5 & 1 & 7 \\ 24 & 1 & 24 & 25 \\ 13 & 3 & 11 & 14 \\ 26 & 3 & 23 & 27 \\ 13 & 3 & 10 & 14 \\ 24 & 0 & 24 & 25 \\ 24 & 1 & 24 & 25 \\ 16 & 2 & 15 & 17 \\ 9 & 4 & 5 & 10 \\ 26 & 2 & 24 & 27 \\ 25 & 5 & 20 & 26 \\ & & & \end{array}$

$\begin{array}{rrrr}8 & 6 & 1295.7839 & 2 \\ 6 & 14 & 1297.4771 & 1 \\ 7 & 9 & 1298.3853 & 1 \\ 6 & 13 & 1299.2650 & 4 \\ 8 & 4 & 1299.2726 & 3 \\ 7 & 8 & 1300.1475 & -7 \\ 8 & 3 & 1300.9962 & 0 \\ 8 & 2 & 1302.7060 & 1 \\ 6 & 11 & 1302.8148 & 0 \\ 7 & 6 & 1303.6398 & 8 \\ 8 & 1 & 1304.4019 & 11 \\ 6 & 10 & 1304.5762 & 0 \\ 6 & 9 & 1306.3283 & 7 \\ 7 & 2 & 1310.4672 & -1 \\ 5 & 11 & 1310.8205 & -4 \\ 6 & 6 & 1311.5156 & -8 \\ 7 & 1 & 1312.1406 & 6 \\ 6 & 5 & 1313.2226 & 1 \\ 5 & 9 & 1314.2908 & 0 \\ 0 & 27 & 1314.7185 & 17 \\ 6 & 4 & 1314.9156 & -5 \\ 3 & 16 & 1315.4659 & -19 \\ 6 & 3 & 1316.5968 & 1 \\ 1 & 26 & 1316.6795 & 4 \\ 0 & 26 & 1316.8585 & 6 \\ 0 & 26 & 1317.0857 & 12 \\ 4 & 12 & 1317.1688 & 9 \\ 5 & 7 & 1317.7217 & -1 \\ 6 & 2 & 1318.2641 & 0 \\ 1 & 25 & 1318.7833 & 8 \\ 4 & 10 & 1318.8391 & -10 \\ 3 & 24 & 1318.8706 & 39 \\ 4 & 11 & 1318.8806 & 9 \\ 0 & 25 & 1318.9805 & 7 \\ 0 & 25 & 1319.2511 & 4 \\ 3 & 14 & 1319.3228 & -22 \\ 5 & 6 & 1319.4204 & -7 \\ 2 & 25 & 1319.5761 & 11 \\ 5 & 21 & 1319.8727 & -78 \\ & & & \\ 7\end{array}$




\begin{tabular}{|c|c|c|c|c|c|c|c|c|}
\hline 16 & 5 & 11 & 16 & 6 & 10 & 1327.6589 & -3 & 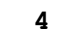 \\
\hline 21 & 9 & 12 & 22 & 9 & 13 & 1327.7195 & 35 & 4 \\
\hline 4 & 4 & 0 & 5 & 5 & 1 & 1327.7346 & -1 & 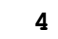 \\
\hline 20 & 1 & 20 & 21 & 0 & 21 & 1327.7961 & 18 & \\
\hline 15 & 5 & 10 & 15 & 6 & 9 & 1327.8678 & 8 & \\
\hline 17 & 1 & 16 & 18 & 2 & 17 & 1327.9165 & 4 & \\
\hline 14 & 5 & 9 & 14 & 6 & 8 & 1328.0647 & -3 & \\
\hline 13 & 5 & 8 & 13 & 6 & 7 & 1328.2520 & -6 & \\
\hline 20 & 2 & 19 & 21 & 2 & 20 & 1328.3484 & -52 & \\
\hline 19 & 0 & 19 & 20 & 1 & 20 & 1328.3558 & 13 & \\
\hline 13 & 1 & 13 & 14 & 2 & 12 & 1328.4160 & 1 & \\
\hline 12 & 5 & 7 & 12 & 6 & 6 & 1328.4290 & -1 & 10 \\
\hline 11 & 2 & 10 & 12 & 3 & 9 & 1328.4290 & -10 & 10 \\
\hline 11 & 5 & 6 & 11 & 6 & 5 & 1328.5955 & 13 & \\
\hline 10 & 5 & 5 & 10 & 6 & 4 & 1328.7483 & 9 & \\
\hline 20 & 3 & 18 & 21 & 3 & 19 & 1328.8590 & -17 & \\
\hline 20 & 4 & 17 & 21 & 4 & 18 & 1328.9609 & -6 & \\
\hline 20 & 4 & 16 & 21 & 4 & 17 & 1328.9766 & 0 & \\
\hline 19 & 1 & 19 & 20 & 1 & 20 & 1328.9895 & 6 & \\
\hline 8 & 5 & 3 & 8 & 6 & 2 & 1329.0162 & 2 & \\
\hline 7 & 3 & 4 & 8 & 4 & 5 & 1329.0281 & -7 & \\
\hline 20 & 5 & 15 & 21 & 5 & 16 & 1329.0412 & -20 & \\
\hline 20 & 3 & 17 & 21 & 3 & 18 & 1329.1131 & 18 & \\
\hline 7 & 5 & 2 & 7 & 6 & 1 & 1329.1312 & 5 & 4 \\
\hline 20 & 6 & 14 & 21 & 6 & 15 & 1329.1595 & -11 & 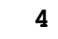 \\
\hline 19 & 0 & 19 & 20 & 0 & 20 & 1329.2650 & 3 & 10 \\
\hline 20 & 7 & 13 & 21 & 7 & 14 & 1329.2650 & 13 & 10 \\
\hline 20 & 1 & 19 & 21 & 1 & 20 & 1329.2979 & -22 & \\
\hline 16 & 1 & 15 & 17 & 2 & 16 & 1329.3627 & 9 & \\
\hline 20 & 8 & 12 & 21 & 8 & 13 & 1329.4697 & -1 & \\
\hline 20 & 9 & 11 & 21 & 9 & 12 & 1329.6212 & 16 & \\
\hline 20 & 2 & 18 & 21 & 2 & 19 & 1329.7062 & 40 & \\
\hline 19 & 1 & 19 & 20 & 0 & 20 & 1329.9003 & 12 & \\
\hline 10 & 2 & 9 & 11 & 3 & 8 & 1330.1770 & 12 & \\
\hline 19 & 2 & 18 & 20 & 2 & 19 & 1330.2169 & -39 & \\
\hline 18 & 0 & 18 & 19 & 1 & 19 & 1330.2228 & 5 & \\
\hline 10 & 2 & 8 & 11 & 3 & 9 & 1330.4941 & 15 & \\
\hline 12 & 1 & 12 & 13 & 2 & 11 & 1330.6442 & 4 & \\
\hline 19 & 3 & 17 & 20 & 3 & 18 & 1330.6574 & -18 & \\
\hline 6 & 3 & 3 & 7 & 4 & 4 & 1330.6876 & 0 & \\
\hline 19 & 4 & 16 & 20 & 4 & 17 & 1330.7586 & -6 & \\
\hline 19 & 4 & 15 & 20 & 4 & 16 & 1330.7693 & 0 & \\
\hline 19 & 3 & 16 & 20 & 3 & 17 & 1330.8477 & 13 & \\
\hline 19 & 5 & 14 & 20 & 5 & 15 & 1330.8556 & -9 & \\
\hline 18 & 1 & 18 & 19 & 1 & 19 & 1330.9654 & 7 & \\
\hline 19 & 6 & 13 & 20 & 6 & 14 & 1330.9898 & -8 & \\
\hline 19 & 7 & 12 & 20 & 7 & 13 & 1331.0832 & 24 & \\
\hline 19 & 1 & 18 & 20 & 1 & 19 & 1331.0939 & -14 & \\
\hline 18 & 0 & 18 & 19 & 0 & 19 & 1331.2491 & 6 & \\
\hline 19 & 8 & 11 & 20 & 8 & 12 & 1331.3387 & 7 & \\
\hline 19 & 2 & 17 & 20 & 2 & 18 & 1331.3742 & 38 & \\
\hline 19 & 9 & 10 & 20 & 9 & 11 & 1331.5144 & 5 & \\
\hline 19 & 10 & 9 & 20 & 10 & 10 & 1331.7642 & -7 & \\
\hline 17 & 0 & 17 & 18 & 1 & 18 & 1332.0549 & 7 & \\
\hline 18 & 2 & 17 & 19 & 2 & 18 & 1332.0670 & -33 & \\
\hline 9 & 2 & 7 & 10 & 3 & 8 & 1332.1133 & 0 & \\
\hline 14 & 1 & 13 & 15 & 2 & 14 & 1332.2384 & 10 & \\
\hline 5 & 3 & 2 & 6 & 4 & 3 & 1332.3342 & -4 & \\
\hline 18 & 3 & 16 & 19 & 3 & 17 & 1332.4483 & 3 & \\
\hline 18 & 4 & 15 & 19 & 4 & 16 & 1332.5488 & -11 & \\
\hline 18 & 4 & 14 & 19 & 4 & 15 & 1332.5564 & -1 & \\
\hline 18 & 3 & 15 & 19 & 3 & 16 & 1332.5865 & 13 & \\
\hline 18 & 5 & 13 & 19 & 5 & 14 & 1332.6616 & -4 & \\
\hline 18 & 6 & 12 & 19 & 6 & 13 & 1332.8110 & -8 & \\
\hline 18 & 1 & 17 & 19 & 1 & 18 & 1332.8674 & -1 & \\
\hline 17 & 1 & 17 & 18 & 1 & 18 & 1332.9187 & 8 & \\
\hline 18 & 2 & 16 & 19 & 2 & 17 & 1333.0373 & 32 & \\
\hline 18 & 8 & 10 & 19 & 8 & 11 & 1333.1957 & -9 & \\
\hline 17 & 0 & 17 & 18 & 0 & 18 & 1333.2056 & 5 & \\
\hline 19 & 4 & 16 & 19 & 5 & 15 & 1333.3113 & -9 & \\
\hline 19 & 4 & 15 & 19 & 5 & 14 & 1333.3264 & -9 & \\
\hline 18 & 9 & 9 & 19 & 9 & 10 & 1333.3974 & -6 & \\
\hline 18 & 4 & 15 & 18 & 5 & 14 & 1333.5268 & -3 & \\
\hline 18 & 4 & 14 & 18 & 5 & 13 & 1333.5370 & -1 & \\
\hline 8 & 2 & 7 & 9 & 3 & 6 & 1333.6073 & 13 & \\
\hline 18 & 10 & 8 & 19 & 10 & 9 & 1333.6723 & -3 & 10 \\
\hline 13 & 1 & 12 & 14 & 2 & 13 & 1333.6723 & -4 & 10 \\
\hline
\end{tabular}

\begin{tabular}{|c|c|c|c|c|c|c|c|c|}
\hline 17 & 4 & 14 & 17 & 5 & 13 & 1333.7365 & 11 & 4 \\
\hline 8 & 2 & 6 & 9 & 3 & 7 & 1333.7428 & 9 & 10 \\
\hline 17 & 4 & 13 & 17 & 5 & 12 & 1333.7428 & 10 & 10 \\
\hline 16 & 0 & 16 & 17 & 1 & 17 & 1333.8505 & 8 & 4 \\
\hline 17 & 2 & 16 & 18 & 2 & 17 & 1333.9003 & -21 & 4 \\
\hline 16 & 4 & 13 & 16 & 5 & 12 & 1333.9362 & -2 & 4 \\
\hline 16 & 4 & 12 & 16 & 5 & 11 & 1333.9410 & 6 & 4 \\
\hline 17 & 1 & 17 & 18 & 0 & 18 & 1334.0695 & 7 & 4 \\
\hline 15 & 4 & 11 & 15 & 5 & 10 & 1334.1310 & -10 & 4 \\
\hline 17 & 3 & 15 & 18 & 3 & 16 & 1334.2271 & -2 & 4 \\
\hline 17 & 3 & 14 & 18 & 3 & 15 & 1334.3269 & 13 & 4 \\
\hline 17 & 4 & 14 & 18 & 4 & 15 & 1334.3319 & -11 & 4 \\
\hline 17 & 4 & 13 & 18 & 4 & 14 & 1334.3374 & 2 & 4 \\
\hline 17 & 5 & 12 & 18 & 5 & 13 & 1334.4586 & -4 & 4 \\
\hline 13 & 4 & 9 & 13 & 5 & 8 & 1334.4912 & 5 & 4 \\
\hline 17 & 1 & 16 & 18 & 1 & 17 & 1334.6196 & 7 & 4 \\
\hline 17 & 6 & 11 & 18 & 6 & 12 & 1334.6225 & -11 & 4 \\
\hline 12 & 4 & 8 & 12 & 5 & 7 & 1334.6572 & 9 & 4 \\
\hline 17 & 2 & 15 & 18 & 2 & 16 & 1334.6992 & 29 & 4 \\
\hline 11 & 4 & 7 & 11 & 5 & 6 & 1334.8110 & -7 & 4 \\
\hline 17 & 7 & 10 & 18 & 7 & 11 & 1334.8417 & -13 & 4 \\
\hline 16 & 1 & 16 & 17 & 1 & 17 & 1334.8488 & 6 & 4 \\
\hline 10 & 1 & 10 & 11 & 2 & 9 & 1334.9037 & 8 & 4 \\
\hline 10 & 4 & 6 & 10 & 5 & 5 & 1334.9572 & 8 & 4 \\
\hline 17 & 8 & 9 & 18 & 8 & 10 & 1335.0437 & -11 & 4 \\
\hline 9 & 4 & 5 & 9 & 5 & 4 & 1335.0904 & 6 & 4 \\
\hline 12 & 1 & 11 & 13 & 2 & 12 & 1335.1107 & 10 & 4 \\
\hline 16 & 0 & 16 & 17 & 0 & 17 & 1335.1345 & 9 & 4 \\
\hline 8 & 4 & 4 & 8 & 5 & 3 & 1335.2116 & 2 & 4 \\
\hline 17 & 9 & 8 & 18 & 9 & 9 & 1335.2704 & -4 & 4 \\
\hline 7 & 2 & 6 & 8 & 3 & 5 & 1335.2932 & 2 & 4 \\
\hline 6 & 4 & 2 & 6 & 5 & 1 & 1335.4170 & -3 & 4 \\
\hline 5 & 4 & 1 & 5 & 5 & 0 & 1335.5014 & 7 & 4 \\
\hline 17 & 10 & 7 & 18 & 10 & 8 & 1335.5692 & -28 & 4 \\
\hline 3 & 3 & 0 & 4 & 4 & 1 & 1335.5904 & -7 & 4 \\
\hline 15 & 0 & 15 & 16 & 1 & 16 & 1335.6103 & 11 & 4 \\
\hline 16 & 2 & 15 & 17 & 2 & 16 & 1335.7158 & -17 & 4 \\
\hline 16 & 3 & 14 & 17 & 3 & 15 & 1335.9968 & -3 & 4 \\
\hline 16 & 3 & 13 & 17 & 3 & 14 & 1336.0669 & 12 & 4 \\
\hline 16 & 4 & 12 & 17 & 4 & 13 & 1336.1094 & -12 & 4 \\
\hline 16 & 1 & 16 & 17 & 0 & 17 & 1336.1324 & 3 & 4 \\
\hline 16 & 5 & 11 & 17 & 5 & 12 & 1336.2466 & -3 & 4 \\
\hline 16 & 1 & 15 & 17 & 1 & 16 & 1336.3520 & 6 & 4 \\
\hline 16 & 2 & 14 & 17 & 2 & 15 & 1336.3611 & 24 & 4 \\
\hline 16 & 6 & 10 & 17 & 6 & 11 & 1336.4248 & -5 & 4 \\
\hline 11 & 1 & 10 & 12 & 2 & 11 & 1336.5504 & 1 & 4 \\
\hline 16 & 7 & 9 & 17 & 7 & 10 & 1336.6472 & -7 & 4 \\
\hline 15 & 1 & 15 & 16 & 1 & 16 & 1336.7562 & 8 & 4 \\
\hline 16 & 8 & 8 & 17 & 8 & 9 & 1336.8808 & -11 & 4 \\
\hline 9 & 1 & 9 & 10 & 2 & 8 & 1336.9401 & -1 & 4 \\
\hline 6 & 2 & 5 & 7 & 3 & 4 & 1336.9620 & -8 & 4 \\
\hline 6 & 2 & 4 & 7 & 3 & 5 & 1337.0080 & -3 & 4 \\
\hline 15 & 0 & 15 & 16 & 0 & 16 & 1337.0339 & 6 & 4 \\
\hline 16 & 9 & 7 & 17 & 9 & 8 & 1337.1302 & -10 & 4 \\
\hline 14 & 0 & 14 & 15 & 1 & 15 & 1337.3343 & 8 & 4 \\
\hline 16 & 10 & 6 & 17 & 10 & 7 & 1337.4572 & -44 & 10 \\
\hline 15 & 2 & 14 & 16 & 2 & 15 & 1337.5149 & -10 & 4 \\
\hline 15 & 3 & 12 & 16 & 3 & 13 & 1337.8059 & 20 & 4 \\
\hline 15 & 4 & 11 & 16 & 4 & 12 & 1337.8752 & -7 & 4 \\
\hline 10 & 1 & 9 & 11 & 2 & 10 & 1337.9962 & -2 & 4 \\
\hline 15 & 5 & 10 & 16 & 5 & 11 & 1338.0249 & -3 & 10 \\
\hline 15 & 2 & 13 & 16 & 2 & 14 & 1338.0249 & 24 & 10 \\
\hline 15 & 1 & 14 & 16 & 1 & 15 & 1338.0672 & 3 & 4 \\
\hline 15 & 1 & 15 & 16 & 0 & 16 & 1338.1804 & 8 & 4 \\
\hline 15 & 6 & 9 & 16 & 6 & 10 & 1338.2162 & -4 & 4 \\
\hline 15 & 7 & 8 & 16 & 7 & 9 & 1338.4490 & -6 & 4 \\
\hline 18 & 2 & 17 & 19 & 1 & 18 & 1338.4760 & -8 & 4 \\
\hline 5 & 2 & 4 & 6 & 3 & 3 & 1338.6160 & -2 & 4 \\
\hline 14 & 1 & 14 & 15 & 1 & 15 & 1338.6399 & 3 & 4 \\
\hline 15 & 8 & 7 & 16 & 8 & 8 & 1338.7067 & -6 & 4 \\
\hline 14 & 0 & 14 & 15 & 0 & 15 & 1338.9043 & 5 & 4 \\
\hline 8 & 1 & 8 & 9 & 2 & 7 & 1338.9201 & 0 & 4 \\
\hline 15 & 9 & 6 & 16 & 9 & 7 & 1338.9757 & -6 & 4 \\
\hline 13 & 0 & 13 & 14 & 1 & 14 & 1339.0262 & 18 & 4 \\
\hline 14 & 2 & 13 & 15 & 2 & 14 & 1339.2971 & -9 & 4 \\
\hline 15 & 10 & 5 & 16 & 10 & 6 & 1339.3367 & -32 & 10 \\
\hline 9 & 1 & 8 & 10 & 2 & 9 & 1339.4501 & 6 & 4 \\
\hline
\end{tabular}




\begin{tabular}{|c|c|c|c|c|c|c|c|}
\hline 14 & 3 & 12 & 15 & 3 & 13 & 1339.5077 & 0 \\
\hline 14 & 3 & 11 & 15 & 3 & 12 & 1339.5390 & 6 \\
\hline 19 & 3 & 17 & 19 & 4 & 16 & 1339.6243 & -22 \\
\hline 14 & 4 & 10 & 15 & 4 & 11 & 1339.6321 & -3 \\
\hline 14 & 2 & 12 & 15 & 2 & 13 & 1339.6901 & 17 \\
\hline 14 & 1 & 13 & 15 & 1 & 14 & 1339.7681 & 10 \\
\hline 14 & 5 & 9 & 15 & 5 & 10 & 1339.7932 & -1 \\
\hline 18 & 3 & 16 & 18 & 4 & 15 & 1339.8428 & -6 \\
\hline 14 & 6 & 8 & 15 & 6 & 9 & 1339.9965 & -4 \\
\hline 17 & 3 & 15 & 17 & 4 & 14 & 1340.0497 & -1 \\
\hline 18 & 3 & 15 & 18 & 4 & 14 & 1340.0962 & 12 \\
\hline 14 & 1 & 14 & 15 & 0 & 15 & 1340.2103 & 4 \\
\hline 17 & 3 & 14 & 17 & 4 & 13 & 1340.2329 & 10 \\
\hline 4 & 2 & 3 & 5 & 3 & 2 & 1340.2532 & -10 \\
\hline 4 & 2 & 2 & 5 & 3 & 3 & 1340.2627 & -13 \\
\hline 16 & 3 & 13 & 16 & 4 & 12 & 1340.3763 & 11 \\
\hline 15 & 3 & 13 & 15 & 4 & 12 & 1340.4335 & 7 \\
\hline 13 & 1 & 13 & 14 & 1 & 14 & 1340.5012 & 5 \\
\hline 14 & 8 & 6 & 15 & 8 & 7 & 1340.5202 & -1 \\
\hline 17 & 2 & 16 & 18 & 1 & 17 & 1340.6037 & -15 \\
\hline 14 & 3 & 12 & 14 & 4 & 11 & 1340.6098 & 1 \\
\hline 14 & 3 & 11 & 14 & 4 & 10 & 1340.6704 & 7 \\
\hline 12 & 0 & 12 & 13 & 1 & 13 & 1340.6841 & 2 \\
\hline 13 & 0 & 13 & 14 & 0 & 14 & 1340.7458 & 6 \\
\hline 13 & 3 & 11 & 13 & 4 & 10 & 1340.7777 & 7 \\
\hline 14 & 9 & 5 & 15 & 9 & 6 & 1340.7949 & 1 \\
\hline 13 & 3 & 10 & 13 & 4 & 9 & 1340.8172 & 10 \\
\hline 7 & 1 & 7 & 8 & 2 & 6 & 1340.8449 & -5 \\
\hline 8 & 1 & 7 & 9 & 2 & 8 & 1340.9122 & 13 \\
\hline 12 & 3 & 10 & 12 & 4 & 9 & 1340.9344 & -2 \\
\hline 12 & 3 & 9 & 12 & 4 & 8 & 1340.9600 & 8 \\
\hline 13 & 2 & 12 & 14 & 2 & 13 & 1341.0641 & -1 \\
\hline 11 & 3 & 9 & 11 & 4 & 8 & 1341.0825 & 3 \\
\hline 11 & 3 & 8 & 11 & 4 & 7 & 1341.0982 & 12 \\
\hline 14 & 10 & 4 & 15 & 10 & 5 & 1341.2021 & -36 \\
\hline 10 & 3 & 8 & 10 & 4 & 7 & 1341.2199 & 4 \\
\hline 10 & 3 & 7 & 10 & 4 & 6 & 1341.2288 & 8 \\
\hline 13 & 3 & 11 & 14 & 3 & 12 & 1341.2485 & 3 \\
\hline 13 & 3 & 10 & 14 & 3 & 11 & 1341.2681 & 4 \\
\hline 9 & 3 & 7 & 9 & 4 & 6 & 1341.3469 & 7 \\
\hline 9 & 3 & 6 & 9 & 4 & 5 & 1341.3518 & 9 \\
\hline 13 & 2 & 11 & 14 & 2 & 12 & 1341.3582 & 18 \\
\hline 13 & 4 & 9 & 14 & 4 & 10 & 1341.3794 & 1 \\
\hline 13 & 1 & 12 & 14 & 1 & 13 & 1341.4547 & 9 \\
\hline 8 & 3 & 5 & 8 & 4 & 4 & 1341.4639 & -4 \\
\hline 13 & 5 & 8 & 14 & 5 & 9 & 1341.5507 & 0 \\
\hline 7 & 3 & 4 & 7 & 4 & 3 & 1341.5671 & -3 \\
\hline 6 & 3 & 3 & 6 & 4 & 2 & 1341.6592 & 0 \\
\hline 5 & 3 & 2 & 5 & 4 & 1 & 1341.7392 & 1 \\
\hline 13 & 6 & 7 & 14 & 6 & 8 & 1341.7653 & -3 \\
\hline 3 & 2 & 1 & 4 & 3 & 2 & 1341.8787 & -19 \\
\hline 13 & 7 & 6 & 14 & 7 & 7 & 1342.0236 & 3 \\
\hline 13 & 1 & 13 & 14 & 0 & 14 & 1342.2222 & 6 \\
\hline 11 & 0 & 11 & 12 & 1 & 12 & 1342.3165 & 13 \\
\hline 13 & 8 & 5 & 14 & 8 & 6 & 1342.3208 & 5 \\
\hline 12 & 1 & 12 & 13 & 1 & 13 & 1342.3392 & 5 \\
\hline 7 & 1 & 6 & 8 & 2 & 7 & 1342.3818 & 1 \\
\hline 12 & 0 & 12 & 13 & 0 & 13 & 1342.5580 & 1 \\
\hline 6 & 1 & 6 & 7 & 2 & 5 & 1342.7184 & -8 \\
\hline 13 & 9 & 4 & 14 & 9 & 5 & 1342.7184 & -13 \\
\hline 12 & 2 & 11 & 13 & 2 & 12 & 1342.8145 & -3 \\
\hline 12 & 3 & 10 & 13 & 3 & 11 & 1342.9789 & 4 \\
\hline 12 & 3 & 9 & 13 & 3 & 10 & 1342.9907 & 3 \\
\hline 12 & 2 & 10 & 13 & 2 & 11 & 1343.0275 & 13 \\
\hline 13 & 10 & 3 & 14 & 10 & 4 & 1343.0558 & -20 \\
\hline 12 & 4 & 8 & 13 & 4 & 9 & 1343.1162 & 0 \\
\hline 12 & 1 & 11 & 13 & 1 & 12 & 1343.1291 & 7 \\
\hline 12 & 5 & 7 & 13 & 5 & 8 & 1343.2968 & -1 \\
\hline 12 & 6 & 6 & 13 & 6 & 7 & 1343.5224 & 2 \\
\hline 12 & 7 & 5 & 13 & 7 & 6 & 1343.7923 & 0 \\
\hline 6 & 1 & 5 & 7 & 2 & 6 & 1343.8627 & -3 \\
\hline 10 & 0 & 10 & 11 & 1 & 11 & 1343.9217 & 1 \\
\hline 12 & 8 & 4 & 13 & 8 & 5 & 1344.1070 & 3 \\
\hline 11 & 1 & 11 & 12 & 1 & 12 & 1344.1541 & 4 \\
\hline 12 & 1 & 12 & 13 & 0 & 13 & 1344.2131 & 4 \\
\hline 11 & 0 & 11 & 12 & 0 & 12 & 1344.3431 & 1 \\
\hline 12 & 9 & 3 & 13 & 9 & 4 & 1344.4839 & 6 \\
\hline
\end{tabular}

\begin{tabular}{|c|c|c|c|c|c|c|c|c|}
\hline 5 & 1 & 5 & 6 & 2 & 4 & 1344.5433 & -5 & \\
\hline 11 & 2 & 10 & 12 & 2 & 11 & 1344.5500 & -1 & \\
\hline 11 & 3 & 9 & 12 & 3 & 10 & 1344.6983 & -1 & 10 \\
\hline 11 & 2 & 9 & 12 & 2 & 10 & 1344.6983 & 13 & 10 \\
\hline 11 & 3 & 8 & 12 & 3 & 9 & 1344.7056 & 3 & \\
\hline 11 & 1 & 10 & 12 & 1 & 11 & 1344.7928 & 7 & \\
\hline 11 & 4 & 7 & 12 & 4 & 8 & 1344.8425 & 1 & \\
\hline 12 & 10 & 2 & 13 & 10 & 3 & 1344.8947 & -4 & \\
\hline 19 & 2 & 18 & 19 & 3 & 17 & 1344.9688 & -37 & \\
\hline 21 & 1 & 21 & 21 & 2 & 20 & 1344.9948 & 7 & \\
\hline 11 & 5 & 6 & 12 & 5 & 7 & 1345.0312 & -2 & \\
\hline 11 & 6 & 5 & 12 & 6 & 6 & 1345.2663 & -1 & \\
\hline 5 & 1 & 4 & 6 & 2 & 5 & 1345.3537 & -17 & \\
\hline 9 & 0 & 9 & 10 & 1 & 10 & 1345.5069 & 1 & \\
\hline 11 & 7 & 4 & 12 & 7 & 5 & 1345.5480 & -3 & \\
\hline 17 & 2 & 16 & 17 & 3 & 15 & 1345.7023 & -27 & \\
\hline 11 & 8 & 3 & 12 & 8 & 4 & 1345.8786 & -2 & \\
\hline 10 & 1 & 10 & 11 & 1 & 11 & 1345.9460 & 2 & \\
\hline 16 & 2 & 15 & 16 & 3 & 14 & 1346.0261 & -10 & \\
\hline 10 & 0 & 10 & 11 & 0 & 11 & 1346.1018 & 2 & \\
\hline 11 & 1 & 11 & 12 & 0 & 12 & 1346.1816 & 1 & \\
\hline 10 & 2 & 9 & 11 & 2 & 10 & 1346.2700 & -4 & \\
\hline 11 & 9 & 2 & 12 & 9 & 3 & 1346.2700 & 13 & 16 \\
\hline 15 & 2 & 14 & 15 & 3 & 13 & 1346.3204 & -10 & 1 \\
\hline 4 & 1 & 4 & 5 & 2 & 3 & 1346.3204 & -13 & 1 \\
\hline 10 & 2 & 8 & 11 & 2 & 9 & 1346.3684 & 8 & \\
\hline 10 & 3 & 8 & 11 & 3 & 9 & 1346.4076 & 1 & \\
\hline 10 & 3 & 7 & 11 & 3 & 8 & 1346.4107 & -6 & \\
\hline 10 & 1 & 9 & 11 & 1 & 10 & 1346.4464 & 3 & \\
\hline 10 & 4 & 6 & 11 & 4 & 7 & 1346.5575 & 1 & \\
\hline 14 & 2 & 13 & 14 & 3 & 12 & 1346.5880 & -14 & \\
\hline 19 & 1 & 19 & 19 & 2 & 18 & 1346.7180 & 13 & 1 \\
\hline 11 & 10 & 1 & 12 & 10 & 2 & 1346.7180 & 15 & 1 \\
\hline 10 & 5 & 5 & 11 & 5 & 6 & 1346.7551 & 14 & \\
\hline 4 & 1 & 3 & 5 & 2 & 4 & 1346. & -11 & \\
\hline 10 & 6 & 4 & 11 & 6 & 5 & 1346.9976 & 1 & \\
\hline 12 & 2 & 11 & 12 & 3 & 10 & 1347.0523 & 8 & \\
\hline 8 & 0 & 8 & 9 & 1 & 9 & 1347.0748 & -1 & \\
\hline 11 & 2 & 10 & 11 & 3 & 9 & 1347.2480 & -4 & \\
\hline 10 & 7 & 3 & 11 & 7 & 4 & 1347.2908 & 3 & \\
\hline 10 & 2 & 9 & 10 & 3 & 8 & 1347.4243 & 3 & \\
\hline 9 & 2 & 8 & 9 & 3 & 7 & 1347.5783 & -12 & \\
\hline 10 & 8 & 2 & 11 & 8 & 3 & 1347.6363 & 2 & \\
\hline 12 & 2 & 10 & 12 & 3 & 9 & 1347.6610 & 16 & \\
\hline 15 & 2 & 13 & 15 & 3 & 12 & 1347.6780 & 20 & \\
\hline 11 & 2 & 9 & 11 & 3 & 8 & 1347.6904 & 11 & \\
\hline 9 & 1 & 9 & 10 & 1 & 10 & 1347.7153 & 1 & \\
\hline 16 & 2 & 14 & 16 & 3 & 13 & 1347.7237 & 30 & \\
\hline 10 & 2 & 8 & 10 & 3 & 7 & 1347.7 & -6 & \\
\hline 9 & 2 & 7 & 9 & 3 & 6 & 1347. & 1 & 1 \\
\hline 17 & 2 & 15 & 17 & 3 & 14 & 1347.7879 & 34 & 1 \\
\hline 9 & 0 & 9 & 10 & 0 & 10 & 1347.8 & -2 & \\
\hline 8 & 2 & 6 & 8 & 3 & 5 & 1347. & -10 & \\
\hline 18 & 2 & 16 & 18 & 3 & 15 & 1347.8688 & 32 & \\
\hline 6 & 2 & 5 & 6 & 3 & 4 & 13 & 4 & \\
\hline 9 & 2 & 8 & 10 & 2 & 9 & 13 & -2 & \\
\hline 6 & 2 & 4 & 6 & 3 & 3 & 1347.9808 & -10 & \\
\hline 5 & 2 & 4 & 5 & 3 & 3 & 1348. & 7 & \\
\hline 9 & 2 & 7 & 10 & 2 & 8 & 1348. & 3 & \\
\hline 5 & 2 & 3 & 5 & 3 & 2 & 1348.0 & -5 & \\
\hline 3 & 1 & 3 & 4 & 2 & 2 & 134 & -4 & \\
\hline 9 & 1 & 8 & 10 & 1 & 9 & & 4 & \\
\hline 9 & 3 & 6 & 10 & 3 & 7 & 1348.1064 & -9 & \\
\hline 10 & 1 & 10 & 11 & 0 & 11 & 13 & 1 & \\
\hline 9 & 4 & 5 & 10 & 4 & 6 & & 0 & \\
\hline 17 & 1 & 17 & 17 & 2 & 16 & 1348. & 4 & \\
\hline 9 & 5 & 4 & 10 & 5 & 5 & 34 & -1 & \\
\hline 7 & 0 & 7 & 8 & 1 & 8 & 95 & -2 & \\
\hline 9 & 6 & 3 & 10 & 6 & 4 & 8.7157 & 4 & \\
\hline 9 & 7 & 2 & 10 & 7 & 3 & 1349.0 & -7 & \\
\hline 9 & 8 & 1 & 10 & 8 & 2 & 91 & 12 & \\
\hline 8 & 1 & 8 & 9 & 1 & 9 & 1349.4619 & -1 & \\
\hline 8 & 0 & 8 & 9 & 0 & 9 & 1349.5461 & -2 & \\
\hline 15 & 1 & 15 & 15 & 2 & 14 & & 1 & \\
\hline 8 & 2 & 7 & 9 & 2 & 8 & 1349.6674 & 0 & \\
\hline 8 & 2 & 6 & 9 & 2 & 7 & 1349.7020 & 2 & \\
\hline 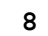 & 1 & 7 & 9 & 1 & 8 & 1349.7289 & 1 & \\
\hline
\end{tabular}




\begin{tabular}{|c|c|c|c|c|c|c|c|}
\hline 8 & 3 & 5 & 9 & 3 & 6 & 1349.7920 & -6 \\
\hline 8 & 4 & 4 & 9 & 4 & 5 & 1349.9515 & -2 \\
\hline 9 & 1 & 9 & 10 & 0 & 10 & 1350.0433 & -5 \\
\hline 8 & 5 & 3 & 9 & 5 & 4 & 1350.1602 & -1 \\
\hline 6 & 0 & 6 & 7 & 1 & 7 & 1350.1746 & -4 \\
\hline 14 & 1 & 14 & 14 & 2 & 13 & 1350.2788 & 18 \\
\hline 8 & 6 & 2 & 9 & 6 & 3 & 1350.4192 & -1 \\
\hline 8 & 7 & 1 & 9 & 7 & 2 & 1350.7325 & 6 \\
\hline 13 & 1 & 13 & 13 & 2 & 12 & 1350.8599 & 11 \\
\hline 8 & 8 & 0 & 9 & 8 & 1 & 1351.1044 & 5 \\
\hline 7 & 1 & 7 & 8 & 1 & 8 & 1351.1857 & -7 \\
\hline 7 & 0 & 7 & 8 & 0 & 8 & 1351.2359 & -1 \\
\hline 7 & 2 & 6 & 8 & 2 & 7 & 1351.3442 & -2 \\
\hline 7 & 2 & 5 & 8 & 2 & 6 & 1351.3602 & -15 \\
\hline 7 & 1 & 6 & 8 & 1 & 7 & 1351.3602 & 12 \\
\hline 12 & 1 & 12 & 12 & 2 & 11 & 1351.3984 & 14 \\
\hline 20 & 0 & 20 & 20 & 1 & 19 & 1351.4405 & 15 \\
\hline 7 & 3 & 4 & 8 & 3 & 5 & 1351.4654 & -11 \\
\hline 7 & 4 & 3 & 8 & 4 & 4 & 1351.6298 & -3 \\
\hline 5 & 0 & 5 & 6 & 1 & 6 & 1351.7138 & -5 \\
\hline 7 & 5 & 2 & 8 & 5 & 3 & 1351.8441 & 4 \\
\hline 11 & 1 & 11 & 11 & 2 & 10 & 1351.8927 & 7 \\
\hline 8 & 1 & 8 & 9 & 0 & 9 & 1351.9326 & -8 \\
\hline 7 & 6 & 1 & 8 & 6 & 2 & 1352.1093 & 2 \\
\hline 10 & 1 & 10 & 10 & 2 & 9 & 1352.3436 & -2 \\
\hline 19 & 0 & 19 & 19 & 1 & 18 & 1352.4892 & 4 \\
\hline 9 & 1 & 9 & 9 & 2 & 8 & 1352.7522 & -7 \\
\hline 6 & 1 & 6 & 7 & 1 & 7 & 1352.8879 & -6 \\
\hline 6 & 0 & 6 & 7 & 0 & 7 & 1352.9060 & -4 \\
\hline 6 & 1 & 5 & 7 & 1 & 6 & 1352.9823 & -3 \\
\hline 6 & 2 & 5 & 7 & 2 & 6 & 1353.0071 & -4 \\
\hline 6 & 2 & 4 & 7 & 2 & 5 & 1353.0149 & 6 \\
\hline 8 & 1 & 8 & 8 & 2 & 7 & 1353.1189 & -7 \\
\hline 6 & 3 & 3 & 7 & 3 & 4 & 1353.1279 & -5 \\
\hline 23 & 1 & 22 & 23 & 2 & 21 & 1353.1900 & -64 \\
\hline 4 & 0 & 4 & 5 & 1 & 5 & 1353.2501 & -6 \\
\hline 6 & 4 & 2 & 7 & 4 & 3 & 1353.2952 & -4 \\
\hline 7 & 1 & 7 & 7 & 2 & 6 & 1353.4442 & 0 \\
\hline 18 & 0 & 18 & 18 & 1 & 17 & 1353.4757 & 3 \\
\hline 6 & 5 & 1 & 7 & 5 & 2 & 1353.5132 & -2 \\
\hline 22 & 1 & 21 & 22 & 2 & 20 & 1353.6398 & -36 \\
\hline 6 & 1 & 6 & 6 & 2 & 5 & 1353.7282 & 11 \\
\hline 6 & 6 & 0 & 7 & 6 & 1 & 1353.7839 & -4 \\
\hline 7 & 1 & 7 & 8 & 0 & 8 & 1353.7921 & -6 \\
\hline 5 & 1 & 5 & 5 & 2 & 4 & 1353.9698 & 10 \\
\hline 21 & 1 & 20 & 21 & 2 & 19 & 1354.0189 & -43 \\
\hline 20 & 1 & 19 & 20 & 2 & 18 & 1354.3380 & -18 \\
\hline 17 & 0 & 17 & 17 & 1 & 16 & 1354.3972 & 8 \\
\hline 5 & 0 & 5 & 6 & 0 & 6 & 1354.5590 & -2 \\
\hline 5 & 1 & 5 & 6 & 1 & 6 & 1354.5674 & -13 \\
\hline 5 & 1 & 4 & 6 & 1 & 5 & 1354.5993 & -9 \\
\hline 3 & 1 & 2 & 3 & 2 & 1 & 1354.6429 & -19 \\
\hline 5 & 2 & 3 & 6 & 2 & 4 & 1354.6570 & -11 \\
\hline 4 & 1 & 3 & 4 & 2 & 2 & 1354.6907 & -15 \\
\hline 10 & 2 & 9 & 11 & 1 & 10 & 1354.7211 & 10 \\
\hline 5 & 1 & 4 & 5 & 2 & 3 & 1354.7479 & -5 \\
\hline 5 & 3 & 2 & 6 & 3 & 3 & 1354.7772 & -4 \\
\hline 3 & 0 & 3 & 4 & 1 & 4 & 1354.7851 & -16 \\
\hline 18 & 1 & 17 & 18 & 2 & 16 & 1354.7990 & -17 \\
\hline 6 & 1 & 5 & 6 & 2 & 4 & 1354.8111 & -2 \\
\hline 7 & $\overline{1}$ & 6 & 7 & 2 & 5 & 1354.8780 & -3 \\
\hline 17 & 1 & 16 & 17 & 2 & 15 & 1354.9548 & 2 \\
\hline 9 & 1 & 8 & 9 & 2 & 7 & 1355.0140 & 12 \\
\hline 16 & 1 & 15 & 16 & 2 & 14 & 1355.0652 & 12 \\
\hline 10 & 1 & 9 & 10 & 2 & 8 & 1355.0727 & -5 \\
\hline 11 & 1 & 10 & 11 & 2 & 9 & 1355.1245 & 9 \\
\hline 15 & 1 & 14 & 15 & 2 & 13 & 1355.1349 & 9 \\
\hline 12 & 1 & 11 & 12 & 2 & 10 & 1355.1604 & 7 \\
\hline 14 & 1 & 13 & 14 & 2 & 12 & 1355.1698 & -1 \\
\hline 5 & 5 & 0 & 6 & 5 & 1 & 1355.1698 & 7 \\
\hline 13 & 1 & 12 & 13 & 2 & 11 & 1355.1773 & 5 \\
\hline 16 & 0 & 16 & 16 & 1 & 15 & 1355.2511 & 11 \\
\hline 6 & 1 & 6 & 7 & 0 & 7 & 1355.6187 & -12 \\
\hline 15 & 0 & 15 & 15 & 1 & 14 & 1356.0358 & 6 \\
\hline 4 & 0 & 4 & 5 & 0 & 5 & 1356.1948 & -10 \\
\hline 4 & 1 & 3 & 5 & 1 & 4 & 1356.2112 & -8 \\
\hline 4 & 1 & 4 & 5 & 1 & 5 & 1356.2265 & -5 \\
\hline
\end{tabular}

\begin{tabular}{|c|c|c|c|c|c|c|c|c|}
\hline 4 & 2 & 2 & 5 & 2 & 3 & 1356.2913 & -1 & 4 \\
\hline 2 & 0 & 2 & 3 & 1 & 3 & 1356.3240 & -3 & 4 \\
\hline 4 & 3 & 1 & 5 & 3 & 2 & 1356.4135 & -4 & 4 \\
\hline 4 & 4 & 0 & 5 & 4 & 1 & 1356.5853 & -9 & 4 \\
\hline 9 & 2 & 8 & 10 & 1 & 9 & 1356.6187 & 7 & 4 \\
\hline 14 & 0 & 14 & 14 & 1 & 13 & 1356.7526 & 6 & 4 \\
\hline 13 & 0 & 13 & 13 & 1 & 12 & 1357.4018 & 7 & 4 \\
\hline 5 & 1 & 5 & 6 & 0 & 6 & 1357.4117 & -19 & 4 \\
\hline 3 & 0 & 3 & 4 & 0 & 4 & 1357.8170 & -5 & 10 \\
\hline 3 & 1 & 2 & 4 & 1 & 3 & 1357.8170 & -14 & 10 \\
\hline 3 & 1 & 3 & 4 & 1 & 4 & 1357.8630 & -5 & 10 \\
\hline 1 & 0 & 1 & 2 & 1 & 2 & 1357.8630 & -22 & 10 \\
\hline 3 & 2 & 1 & 4 & 2 & 2 & 1357.9128 & -2 & 4 \\
\hline 12 & 0 & 12 & 12 & 1 & 11 & 1357.9845 & 5 & 4 \\
\hline 3 & 3 & 0 & 4 & 3 & 1 & 1358.0360 & -9 & 4 \\
\hline 11 & 0 & 11 & 11 & 1 & 10 & 1358.5035 & 4 & 4 \\
\hline 10 & 0 & 10 & 10 & 1 & 9 & 1358.9620 & 6 & 4 \\
\hline 4 & 1 & 4 & 5 & 0 & 5 & 1359.1707 & -14 & 4 \\
\hline 9 & 0 & 9 & 9 & 1 & 8 & 1359.3627 & 3 & 4 \\
\hline 2 & 1 & 1 & 3 & 1 & 2 & 1359.4186 & -10 & 4 \\
\hline 2 & 0 & 2 & 3 & 0 & 3 & 1359.4240 & -10 & 4 \\
\hline 2 & 1 & 2 & 3 & 1 & 3 & 1359.4780 & -3 & 4 \\
\hline 2 & 2 & 0 & 3 & 2 & 1 & 1359.5220 & 1 & 4 \\
\hline 8 & 0 & 8 & 8 & 1 & 7 & 1359.7095 & -2 & 4 \\
\hline 7 & 0 & 7 & 7 & 1 & 6 & 1360.0067 & -4 & 4 \\
\hline 6 & 0 & 6 & 6 & 1 & 5 & 1360.2579 & -5 & 4 \\
\hline 5 & 0 & 5 & 5 & 1 & 4 & 1360.4664 & -6 & 4 \\
\hline 4 & 0 & 4 & 4 & 1 & 3 & 1360.6363 & 4 & 4 \\
\hline 2 & 0 & 2 & 2 & 1 & 1 & 1360.8643 & -5 & 4 \\
\hline 1 & 0 & 1 & 1 & 1 & 0 & 1360.9284 & -1 & 4 \\
\hline 1 & 0 & 1 & 2 & 0 & 2 & 1361.0175 & -17 & 4 \\
\hline 13 & 4 & 9 & 13 & 4 & 10 & 1363.3167 & 4 & 4 \\
\hline 16 & 8 & 8 & 16 & 8 & 9 & 1363.4818 & -12 & 10 \\
\hline 13 & 5 & 8 & 13 & 5 & 9 & 1363.4818 & 8 & 10 \\
\hline 12 & 4 & 8 & 12 & 4 & 9 & 1363.4860 & -13 & 10 \\
\hline 14 & 6 & 8 & 14 & 6 & 9 & 1363.4860 & 3 & 10 \\
\hline 11 & 4 & 7 & 11 & 4 & 8 & 1363.6477 & 3 & 4 \\
\hline 12 & 5 & 7 & 12 & 5 & 8 & 1363.6614 & -9 & 10 \\
\hline 13 & 6 & 7 & 13 & 6 & 8 & 1363.6911 & 6 & 4 \\
\hline 16 & 9 & 7 & 16 & 9 & 8 & 1363.7241 & -7 & 4 \\
\hline 15 & 8 & 7 & 15 & 8 & 8 & 1363.7454 & -12 & 4 \\
\hline 9 & 3 & 6 & 9 & 3 & 7 & 1363.7855 & -6 & 4 \\
\hline 10 & 4 & 6 & 10 & 4 & 7 & 1363.7969 & 9 & 4 \\
\hline 11 & 5 & 6 & 11 & 5 & 7 & 1363.8313 & -3 & 4 \\
\hline 12 & 6 & 6 & 12 & 6 & 7 & 1363.8824 & -5 & 4 \\
\hline 8 & 3 & 5 & 8 & 3 & 6 & 1363.9019 & -9 & 10 \\
\hline 5 & 2 & 4 & 6 & 1 & 5 & 1363.9019 & 3 & 10 \\
\hline 9 & 4 & 5 & 9 & 4 & 6 & 1363.9338 & 10 & 4 \\
\hline 13 & 7 & 6 & 13 & 7 & 7 & 1363.9440 & 9 & 4 \\
\hline 10 & 5 & 5 & 10 & 5 & 6 & 1363.9884 & 0 & 4 \\
\hline 14 & 8 & 6 & 14 & 8 & 7 & 1363.9975 & 1 & 4 \\
\hline 7 & 3 & 4 & 7 & 3 & 5 & 1364.0080 & -5 & 10 \\
\hline 15 & 9 & 6 & 15 & 9 & 7 & 1364.0080 & -7 & 10 \\
\hline 8 & 4 & 4 & 8 & 4 & 5 & 1364.0566 & -5 & 4 \\
\hline 11 & 6 & 5 & 11 & 6 & 6 & 1364.0623 & -1 & 4 \\
\hline 6 & 3 & 3 & 6 & 3 & 4 & 1364.1012 & -11 & 4 \\
\hline 4 & 2 & 3 & 4 & 2 & 2 & 1364.1238 & -12 & 4 \\
\hline 9 & 5 & 4 & 9 & 5 & 5 & 1364.1324 & 1 & 4 \\
\hline 12 & 7 & 5 & 12 & 7 & 6 & 1364.1488 & 5 & 4 \\
\hline 7 & 4 & 3 & 7 & 4 & 4 & 1364.1693 & 6 & 4 \\
\hline 3 & 2 & 2 & 3 & 2 & 1 & 1364.1830 & -1 & 10 \\
\hline 5 & 3 & 2 & 5 & 3 & 3 & 1364.1830 & -8 & 10 \\
\hline 2 & 2 & 1 & 2 & 2 & 0 & 1364.2251 & -4 & 4 \\
\hline 13 & 8 & 5 & 13 & 8 & 6 & 1364.2347 & 1 & 4 \\
\hline 4 & 3 & 1 & 4 & 3 & 2 & 1364.2519 & -5 & 4 \\
\hline 6 & 4 & 2 & 6 & 4 & 3 & 1364.2656 & -16 & 10 \\
\hline 14 & 9 & 5 & 14 & 9 & 6 & 1364.2656 & 2 & 10 \\
\hline 3 & 3 & 0 & 3 & 3 & 1 & 1364.3072 & -5 & 4 \\
\hline 11 & 7 & 4 & 11 & 7 & 5 & 1364.3415 & 15 & 4 \\
\hline 5 & 4 & 1 & 5 & 4 & 2 & 1364.3520 & -1 & 4 \\
\hline 9 & 6 & 3 & 9 & 6 & 4 & 1364.3804 & -5 & 10 \\
\hline 7 & 5 & 2 & 7 & 5 & 3 & 1364.3804 & 4 & 10 \\
\hline 4 & 4 & 0 & 4 & 4 & 1 & 1364.4230 & -4 & 4 \\
\hline 12 & 8 & 4 & 12 & 8 & 5 & 1364.4578 & 2 & 4 \\
\hline 6 & 5 & 1 & 6 & 5 & 2 & 1364.4830 & -1 & 4 \\
\hline 5 & 5 & 0 & 5 & 5 & 1 & 1364.5717 & -4 & 4 \\
\hline 13 & 9 & 4 & 13 & 9 & 5 & 1364.6272 & -8 & 4 \\
\hline
\end{tabular}




\begin{tabular}{|c|c|c|c|c|c|c|c|}
\hline 5 & 2 & 4 & 4 & 2 & 3 & 1371.8971 & -7 \\
\hline 5 & 2 & 3 & 4 & 2 & 2 & 1371.9154 & -4 \\
\hline 5 & 3 & 2 & 4 & 3 & 1 & 1372.0221 & -1 \\
\hline 12 & 2 & 10 & 12 & 1 & 11 & 1372.0221 & 9 \\
\hline 5 & 1 & 4 & 4 & 1 & 3 & 1372.0386 & -3 \\
\hline 5 & 4 & 1 & 4 & 4 & 0 & 1372.1886 & -8 \\
\hline 11 & 2 & 9 & 11 & 1 & 10 & 1372.2341 & 6 \\
\hline 10 & 2 & 8 & 10 & 1 & 9 & 1372.4509 & 5 \\
\hline 11 & 1 & 10 & 10 & 2 & 9 & 1372.5648 & 3 \\
\hline 9 & 2 & 7 & 9 & 1 & 8 & 1372.6680 & 10 \\
\hline 8 & 2 & 6 & 8 & 1 & 7 & 1372.8784 & -1 \\
\hline 6 & 1 & 6 & 5 & 1 & 5 & 1373.0250 & -5 \\
\hline 7 & 2 & 5 & 7 & 1 & 6 & 1373.0795 & -5 \\
\hline 6 & 0 & 6 & 5 & 0 & 5 & 1373.2571 & 1 \\
\hline 6 & 2 & 4 & 6 & 1 & 5 & 1373.2674 & 3 \\
\hline 4 & 1 & 4 & 3 & 0 & 3 & 1373.2707 & -16 \\
\hline 6 & 2 & 5 & 5 & 2 & 4 & 1373.3803 & -6 \\
\hline 8 & 0 & 8 & 7 & 1 & 7 & 1373.3980 & -5 \\
\hline 6 & 2 & 4 & 5 & 2 & 3 & 1373.4154 & 0 \\
\hline 5 & 2 & 3 & 5 & 1 & 4 & 1373.4361 & 5 \\
\hline 6 & 3 & 3 & 5 & 3 & 2 & 1373.5077 & -7 \\
\hline 4 & 2 & 2 & 4 & 1 & 3 & 1373.5805 & -13 \\
\hline 6 & 1 & 5 & 5 & 1 & 4 & 1373.5884 & -4 \\
\hline 6 & 4 & 2 & 5 & 4 & 1 & 1373.6714 & -3 \\
\hline 3 & 2 & 1 & 3 & 1 & 2 & 1373.7013 & -13 \\
\hline 6 & 5 & 1 & 5 & 5 & 0 & 1373.8856 & -5 \\
\hline 3 & 2 & 2 & 3 & 1 & 3 & 1373.9154 & -8 \\
\hline 4 & 2 & 3 & 4 & 1 & 4 & 1373.9338 & 3 \\
\hline 6 & 2 & 5 & 6 & 1 & 6 & 1373.9805 & -2 \\
\hline 7 & 2 & 6 & 7 & 1 & 7 & 1374.0087 & -17 \\
\hline 8 & 2 & 7 & 8 & 1 & 8 & 1374.0439 & -1 \\
\hline 9 & 2 & 8 & 9 & 1 & 9 & 1374.0805 & -8 \\
\hline 10 & 2 & 9 & 10 & 1 & 10 & 1374.1219 & -2 \\
\hline 11 & 2 & 10 & 11 & 1 & 11 & 1374.1664 & 1 \\
\hline 12 & 2 & 11 & 12 & 1 & 12 & 1374.2130 & -4 \\
\hline 12 & 1 & 11 & 11 & 2 & 10 & 1374.2469 & 3 \\
\hline 13 & 2 & 12 & 13 & 1 & 13 & 1374.2627 & -5 \\
\hline 14 & 2 & 13 & 14 & 1 & 14 & 1374.3141 & -13 \\
\hline 15 & 2 & 14 & 15 & 1 & 15 & 1374.3680 & -14 \\
\hline 7 & 1 & 7 & 6 & 1 & 6 & 1374.4170 & -4 \\
\hline 16 & 2 & 15 & 16 & 1 & 16 & 1374.4247 & -2 \\
\hline 27 & 3 & 24 & 27 & 2 & 25 & 1374.4405 & 49 \\
\hline 17 & 2 & 16 & 17 & 1 & 17 & 1374.4791 & -22 \\
\hline 18 & 2 & 17 & 18 & 1 & 18 & 1374.5350 & -31 \\
\hline 19 & 2 & 18 & 19 & 1 & 19 & 1374.5907 & -39 \\
\hline 5 & 1 & 5 & 4 & 0 & 4 & 1374.6409 & 1 \\
\hline 7 & 0 & 7 & 6 & 0 & 6 & 1374.7057 & 1 \\
\hline 26 & 3 & 23 & 26 & 2 & 24 & 1374.7481 & 27 \\
\hline 7 & 2 & 6 & 6 & 2 & 5 & 1374.8487 & -3 \\
\hline 7 & 2 & 5 & 6 & 2 & 4 & 1374.9082 & -5 \\
\hline 9 & 0 & 9 & 8 & 1 & 8 & 1374.9219 & 7 \\
\hline 7 & 3 & 4 & 6 & 3 & 3 & 1374.9814 & -6 \\
\hline 25 & 3 & 22 & 25 & 2 & 23 & 1375.0757 & 28 \\
\hline 7 & 1 & 6 & 6 & 1 & 5 & 1375.1313 & 2 \\
\hline 7 & 4 & 3 & 6 & 4 & 2 & 1375.1400 & -3 \\
\hline 7 & 5 & 2 & 6 & 5 & 1 & 1375.3498 & 1 \\
\hline 24 & 3 & 21 & 24 & 2 & 22 & 1375.4171 & 27 \\
\hline 7 & 6 & 1 & 6 & 6 & 0 & 1375.6094 & -14 \\
\hline 23 & 3 & 20 & 23 & 2 & 21 & 1375.7694 & 32 \\
\hline 8 & 1 & 8 & 7 & 1 & 7 & 1375.7853 & -2 \\
\hline 6 & 1 & 6 & 5 & 0 & 5 & 1375.9697 & -9 \\
\hline 8 & 0 & 8 & 7 & 0 & 7 & 1376.1293 & -6 \\
\hline 8 & 2 & 7 & 7 & 2 & 6 & 1376.3015 & -3 \\
\hline 8 & 2 & 6 & 7 & 2 & 5 & 1376.3982 & 3 \\
\hline 10 & 0 & 10 & 9 & 1 & 9 & 1376.4244 & -4 \\
\hline 8 & 3 & 5 & 7 & 3 & 4 & 1376.4428 & -9 \\
\hline 21 & 3 & 18 & 21 & 2 & 19 & 1376.4846 & 16 \\
\hline 8 & 4 & 4 & 7 & 4 & 3 & 1376.5958 & 1 \\
\hline 8 & 1 & 7 & 7 & 1 & 6 & 1376.6651 & 1 \\
\hline 20 & 3 & 17 & 20 & 2 & 18 & 1376.8401 & 8 \\
\hline 8 & 6 & 2 & 7 & 6 & 1 & 1377.0534 & 3 \\
\hline 9 & 1 & 9 & 8 & 1 & 8 & 1377.1295 & 0 \\
\hline 19 & 3 & 16 & 19 & 2 & 17 & 1377.1900 & 13 \\
\hline 7 & 1 & 7 & 6 & 0 & 6 & 1377.2632 & 10 \\
\hline 9 & 0 & 9 & 8 & 0 & 8 & 1377.5281 & 6 \\
\hline 18 & 3 & 15 & 18 & 2 & 16 & 1377.5281 & 7 \\
\hline 14 & 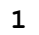 & 13 & 13 & 2 & 12 & 377.6133 & 5 \\
\hline
\end{tabular}




\begin{tabular}{|c|c|c|c|c|c|c|c|c|}
\hline 11 & 8 & 3 & 10 & 8 & 2 & 1381.8912 & 21 & 4 \\
\hline 12 & 2 & 11 & 11 & 2 & 10 & 1381.9515 & -1 & 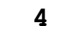 \\
\hline 11 & 1 & 11 & 10 & 0 & 10 & 1382.0725 & 2 & \\
\hline 12 & 3 & 10 & 11 & 3 & 9 & 1382.1666 & 6 & \\
\hline 14 & 0 & 14 & 13 & 1 & 13 & 1382.1716 & 17 & \\
\hline 12 & 3 & 9 & 11 & 3 & 8 & 1382.1866 & 7 & \\
\hline 13 & 1 & 13 & 12 & 1 & 12 & 1382.2582 & 8 & \\
\hline 11 & 9 & 2 & 10 & 9 & 1 & 1382.2713 & 18 & \\
\hline 12 & 4 & 8 & 11 & 4 & 7 & 1382.2922 & 1 & \\
\hline 12 & 2 & 10 & 11 & 2 & 9 & 1382.3540 & 13 & \\
\hline 12 & 5 & 7 & 11 & 5 & 6 & 1382.4629 & 4 & \\
\hline 17 & 1 & 16 & 16 & 2 & 15 & 1382.6299 & 2 & \\
\hline 12 & 6 & 6 & 11 & 6 & 5 & 1382.6789 & 0 & \\
\hline 12 & 1 & 11 & 11 & 1 & 10 & 1382.6968 & 6 & \\
\hline 6 & 2 & 5 & 5 & 1 & 4 & 1382.7338 & 4 & \\
\hline 13 & 0 & 13 & 12 & 0 & 12 & 1382.8094 & 6 & \\
\hline 24 & 4 & 20 & 24 & 3 & 21 & 1382.8295 & 1 & \\
\hline 12 & 7 & 5 & 11 & 7 & 4 & 1382.9403 & 2 & \\
\hline 23 & 4 & 19 & 23 & 3 & 20 & 1383.1606 & -1 & \\
\hline 12 & 1 & 12 & 11 & 0 & 11 & 1383.1937 & 5 & 0 \\
\hline 12 & 8 & 4 & 11 & 8 & 3 & 1383.2452 & 3 & \\
\hline 13 & 2 & 12 & 12 & 2 & 11 & 1383.3210 & -5 & \\
\hline 23 & 4 & 20 & 23 & 3 & 21 & 1383.4417 & -29 & \\
\hline 14 & 1 & 14 & 13 & 1 & 13 & 1383.4768 & 8 & \\
\hline 15 & 0 & 15 & 14 & 1 & 14 & 1383.5235 & 7 & \\
\hline 13 & 3 & 11 & 12 & 3 & 10 & 1383.5694 & 4 & \\
\hline 13 & 3 & 10 & 12 & 3 & 9 & 1383.6009 & 6 & \\
\hline 12 & 9 & 3 & 11 & 9 & 2 & 1383.6114 & 3 & \\
\hline 13 & 4 & 9 & 12 & 4 & 8 & 1383.6868 & -3 & \\
\hline 22 & 4 & 19 & 22 & 3 & 20 & 1383.7010 & -18 & \\
\hline 21 & 4 & 17 & 21 & 3 & 18 & 1383.7820 & -1 & \\
\hline 13 & 5 & 8 & 12 & 5 & 7 & 1383.8468 & 4 & \\
\hline 13 & 2 & 11 & 12 & 2 & 10 & 1383.8511 & 19 & \\
\hline 21 & 4 & 18 & 21 & 3 & 19 & 1383.9552 & -18 & \\
\hline 14 & 0 & 14 & 13 & 0 & 13 & 1384.0438 & -1 & \\
\hline 13 & 6 & 7 & 12 & 6 & 6 & 1384.0512 & 0 & \\
\hline 20 & 4 & 16 & 20 & 3 & 17 & 1384.0721 & 4 & \\
\hline 7 & 2 & 6 & 6 & 1 & 5 & 1384.0939 & 1 & \\
\hline 13 & 1 & 12 & 12 & 1 & 11 & 1384.1724 & 6 & \\
\hline 20 & 4 & 17 & 20 & 3 & 18 & 1384.2059 & -1 & \\
\hline 13 & 1 & 13 & 12 & 0 & 12 & 1384.2854 & 3 & \\
\hline 13 & 7 & 6 & 12 & 7 & 5 & 1384.2989 & -1 & \\
\hline 19 & 4 & 15 & 19 & 3 & 16 & 1384.3474 & 0 & \\
\hline 19 & 4 & 16 & 19 & 3 & 17 & 1384.4484 & -5 & \\
\hline 13 & 8 & 5 & 12 & 8 & 4 & 1384.5855 & 0 & \\
\hline 18 & 4 & 14 & 18 & 3 & 15 & 1384.6091 & -3 & \\
\hline 14 & 2 & 13 & 13 & 2 & 12 & 1384.6717 & -17 & 10 \\
\hline 15 & 1 & 15 & 14 & 1 & 14 & 1384.6717 & 27 & 10 \\
\hline 18 & 4 & 15 & 18 & 3 & 16 & 1384.6846 & -1 & \\
\hline 16 & 0 & 16 & 15 & 1 & 15 & 1384.8387 & 8 & \\
\hline 17 & 4 & 13 & 17 & 3 & 14 & 1384.8586 & 8 & \\
\hline 17 & 4 & 14 & 17 & 3 & 15 & 1384.9126 & 0 & \\
\hline 7 & 2 & 5 & 6 & 1 & 6 & 1384.9352 & 16 & \\
\hline 14 & 3 & 12 & 13 & 3 & 11 & 1384.9625 & 13 & \\
\hline 13 & 9 & 4 & 12 & 9 & 3 & 1384.9727 & -8 & \\
\hline 14 & 3 & 11 & 13 & 3 & 10 & 1385.0092 & 5 & \\
\hline 3 & 3 & 0 & 2 & 2 & 1 & 1385.0467 & -6 & \\
\hline 14 & 4 & 10 & 13 & 4 & 9 & 1385.0708 & -6 & \\
\hline 16 & 4 & 12 & 16 & 3 & 13 & 1385.0924 & -4 & \\
\hline 16 & 4 & 13 & 16 & 3 & 14 & 1385.1319 & 1 & \\
\hline 14 & 5 & 9 & 13 & 5 & 8 & 1385.2182 & -2 & \\
\hline 15 & 0 & 15 & 14 & 0 & 14 & 1385.2440 & 4 & \\
\hline 15 & 4 & 11 & 15 & 3 & 12 & 1385.3144 & -1 & \\
\hline 15 & 4 & 12 & 15 & 3 & 13 & 1385.3425 & 9 & \\
\hline 14 & 2 & 12 & 13 & 2 & 11 & 1385.3516 & 11 & 10 \\
\hline 14 & 1 & 14 & 13 & 0 & 13 & 1385.3516 & 16 & 10 \\
\hline 14 & 6 & 8 & 13 & 6 & 7 & 1385.4101 & -5 & \\
\hline 8 & 2 & 7 & 7 & 1 & 6 & 1385.4210 & -5 & \\
\hline 14 & 4 & 10 & 14 & 3 & 11 & 1385.5232 & 2 & \\
\hline 14 & 4 & 11 & 14 & 3 & 12 & 1385.5413 & 0 & \\
\hline 14 & 1 & 13 & 13 & 1 & 12 & 1385.6315 & 1 & \\
\hline 14 & 7 & 7 & 13 & 7 & 6 & 1385.6445 & -3 & \\
\hline 13 & 4 & 9 & 13 & 3 & 10 & 1385.7187 & 4 & \\
\hline 13 & 4 & 10 & 13 & 3 & 11 & 1385.7312 & 9 & \\
\hline 16 & 1 & 16 & 15 & 1 & 15 & 1385.8369 & 6 & \\
\hline 12 & 4 & 8 & 12 & 3 & 9 & 1385.9005 & 1 & \\
\hline 12 & 4 & 9 & 12 & 3 & 10 & 1385.9097 & 18 & \\
\hline
\end{tabular}




\begin{tabular}{|c|c|c|c|c|c|c|c|}
\hline 15 & 2 & 14 & 14 & 2 & 13 & 1386.0055 & -13 \\
\hline 11 & 4 & 7 & 11 & 3 & 8 & 1386.0702 & 11 \\
\hline 11 & 4 & 8 & 11 & 3 & 9 & 1386.0736 & -1 \\
\hline 17 & 0 & 17 & 16 & 1 & 16 & 1386.1140 & -2 \\
\hline 14 & 9 & 5 & 13 & 9 & 4 & 1386.1741 & 4 \\
\hline 10 & 4 & 6 & 10 & 3 & 7 & 1386.2260 & 14 \\
\hline 15 & 3 & 13 & 14 & 3 & 12 & 1386.3430 & 3 \\
\hline 9 & 4 & 5 & 9 & 3 & 6 & 1386.3673 & 8 \\
\hline 15 & 1 & 15 & 14 & 0 & 14 & 1386.3906 & 7 \\
\hline 16 & 0 & 16 & 15 & 0 & 15 & 1386.4087 & 5 \\
\hline 15 & 3 & 12 & 14 & 3 & 11 & 1386.4135 & 9 \\
\hline 15 & 4 & 11 & 14 & 4 & 10 & 1386.4446 & -12 \\
\hline 8 & 4 & 4 & 8 & 3 & 5 & 1386.4951 & 2 \\
\hline 4 & 3 & 1 & 3 & 2 & 2 & 1386.5584 & -13 \\
\hline 15 & 5 & 10 & 14 & 5 & 9 & 1386.5789 & 0 \\
\hline 7 & 4 & 3 & 7 & 3 & 4 & 1386.6094 & -1 \\
\hline 9 & 2 & 8 & 8 & 1 & 7 & 1386.7162 & 1 \\
\hline 15 & 6 & 9 & 14 & 6 & 8 & 1386.7577 & 1 \\
\hline 5 & 4 & 1 & 5 & 3 & 2 & 1386.7969 & 1 \\
\hline 15 & 2 & 13 & 14 & 2 & 12 & 1386.8589 & 23 \\
\hline 4 & 4 & 0 & 4 & 3 & 1 & 1386.8687 & -6 \\
\hline 15 & 7 & 8 & 14 & 7 & 7 & 1386.9781 & -3 \\
\hline 17 & 1 & 17 & 16 & 1 & 16 & 1386.9781 & 2 \\
\hline 15 & 1 & 14 & 14 & 1 & 13 & 1387.0738 & 6 \\
\hline 15 & 8 & 7 & 14 & 8 & 6 & 1387.2233 & -4 \\
\hline 16 & 2 & 15 & 15 & 2 & 14 & 1387.3195 & -17 \\
\hline 18 & 0 & 18 & 17 & 1 & 17 & 1387.3521 & 6 \\
\hline 16 & 1 & 16 & 15 & 0 & 15 & 1387.4073 & 7 \\
\hline 15 & 9 & 6 & 14 & 9 & 5 & 1387.4791 & -2 \\
\hline 17 & 0 & 17 & 16 & 0 & 16 & 1387.5399 & 15 \\
\hline 16 & 3 & 14 & 15 & 3 & 13 & 1387.7132 & -3 \\
\hline 16 & 4 & 12 & 15 & 4 & 11 & 1387.8084 & -25 \\
\hline 16 & 3 & 13 & 15 & 3 & 12 & 1387.8134 & -4 \\
\hline 16 & 5 & 11 & 15 & 5 & 10 & 1387.9281 & -4 \\
\hline 10 & 2 & 9 & 9 & 1 & 8 & 1387.9782 & 5 \\
\hline 5 & 3 & 3 & 4 & 2 & 2 & 1388.0554 & 10 \\
\hline 5 & 3 & 2 & 4 & 2 & 3 & 1388.0599 & 7 \\
\hline 18 & 1 & 18 & 17 & 1 & 17 & 1388.0931 & -8 \\
\hline 16 & 6 & 10 & 15 & 6 & 9 & 1388.0931 & 5 \\
\hline 9 & 2 & 7 & 8 & 1 & 8 & 1388.2271 & 13 \\
\hline 16 & 7 & 9 & 15 & 7 & 8 & 1388.3010 & -8 \\
\hline 16 & 2 & 14 & 15 & 2 & 13 & 1388.3694 & 28 \\
\hline 17 & 1 & 17 & 16 & 0 & 16 & 1388.4027 & 7 \\
\hline 16 & 1 & 15 & 15 & 1 & 14 & 1388.4958 & 5 \\
\hline 16 & 8 & 8 & 15 & 8 & 7 & 1388.5231 & 8 \\
\hline 19 & 0 & 19 & 18 & 1 & 18 & 1388.5524 & 23 \\
\hline 17 & 2 & 16 & 16 & 2 & 15 & 1388.6133 & -27 \\
\hline 18 & 0 & 18 & 17 & 0 & 17 & 1388.6365 & 10 \\
\hline 16 & 9 & 7 & 15 & 9 & 6 & 1388.7575 & 3 \\
\hline 17 & 3 & 15 & 16 & 3 & 14 & 1389.0729 & -8 \\
\hline 17 & 4 & 14 & 16 & 4 & 13 & 1389.1618 & 1 \\
\hline 17 & 4 & 13 & 16 & 4 & 12 & 1389.1680 & 8 \\
\hline 19 & 1 & 19 & 18 & 1 & 18 & 1389.1860 & 15 \\
\hline 11 & 2 & 10 & 10 & 1 & 9 & 1389.2063 & 2 \\
\hline 17 & 3 & 14 & 16 & 3 & 13 & 1389.2148 & 6 \\
\hline 17 & 5 & 12 & 16 & 5 & 11 & 1389.2670 & -5 \\
\hline 17 & 6 & 11 & 16 & 6 & 10 & 1389.4153 & -8 \\
\hline 6 & 3 & 4 & 5 & 2 & 3 & 1389.5358 & 4 \\
\hline 6 & 3 & 3 & 5 & 2 & 4 & 1389.5463 & -2 \\
\hline 17 & 7 & 10 & 16 & 7 & 9 & 1389.6200 & -10 \\
\hline 19 & 0 & 19 & 18 & 0 & 18 & 1389.7021 & 11 \\
\hline 20 & 0 & 20 & 19 & 1 & 19 & 1389.7113 & 7 \\
\hline 17 & 8 & 9 & 16 & 8 & 8 & 1389.8081 & 1 \\
\hline 17 & 2 & 15 & 16 & 2 & 14 & 1389.8819 & 28 \\
\hline 18 & 2 & 17 & 17 & 2 & 16 & 1389.8874 & -33 \\
\hline 17 & 1 & 16 & 16 & 1 & 15 & 1389.8952 & -1 \\
\hline 17 & 9 & 8 & 16 & 9 & 7 & 1390.0189 & 2 \\
\hline 20 & 1 & 20 & 19 & 1 & 19 & 1390.2510 & 10 \\
\hline 17 & 10 & 7 & 16 & 10 & 6 & 1390.2997 & -37 \\
\hline 19 & 1 & 19 & 18 & 0 & 18 & 1390.3379 & 25 \\
\hline 12 & 2 & 11 & 11 & 1 & 10 & 1390.4015 & 2 \\
\hline 18 & 3 & 16 & 17 & 3 & 15 & 1390.4219 & -11 \\
\hline 18 & 4 & 15 & 17 & 4 & 14 & 1390.5068 & -3 \\
\hline 18 & 4 & 14 & 17 & 4 & 13 & 1390.5157 & 0 \\
\hline 18 & 5 & 13 & 17 & 5 & 12 & 1390.5955 & -10 \\
\hline 18 & 3 & 15 & 17 & 3 & 14 & 1390.6170 & 14 \\
\hline 20 & 5 & 15 & 20 & 4 & 16 & 1390.6679 & -8 \\
\hline
\end{tabular}

\begin{tabular}{|c|c|c|c|c|c|c|c|}
\hline 18 & 6 & 12 & 17 & 6 & 11 & 1390.7280 & -6 \\
\hline 20 & 0 & 20 & 19 & 0 & 19 & 1390.7370 & 3 \\
\hline 21 & 0 & 21 & 20 & 1 & 20 & 1390.8362 & 21 \\
\hline 19 & 5 & 14 & 19 & 4 & 15 & 1390.9361 & 7 \\
\hline 7 & 3 & 5 & 6 & 2 & 4 & 1390.9995 & -6 \\
\hline 7 & 3 & 4 & 6 & 2 & 5 & 1391.0227 & 2 \\
\hline 18 & 8 & 10 & 17 & 8 & 9 & 1391.0829 & 14 \\
\hline 19 & 2 & 18 & 18 & 2 & 17 & 1391.1397 & -51 \\
\hline 18 & 5 & 13 & 18 & 4 & 14 & 1391.1922 & 3 \\
\hline 18 & 9 & 9 & 17 & 9 & 8 & 1391.2658 & -6 \\
\hline 18 & 1 & 17 & 17 & 1 & 16 & 1391.2701 & -9 \\
\hline 20 & 1 & 20 & 19 & 0 & 19 & 1391.2776 & 15 \\
\hline 21 & 1 & 21 & 20 & 1 & 20 & 1391.2921 & 15 \\
\hline 18 & 2 & 16 & 17 & 2 & 15 & 1391.3959 & 36 \\
\hline 17 & 5 & 12 & 17 & 4 & 13 & 1391.4378 & 2 \\
\hline 18 & 10 & 8 & 17 & 10 & 7 & 1391.5210 & -24 \\
\hline 13 & 2 & 12 & 12 & 1 & 11 & 1391.5634 & 1 \\
\hline 16 & 5 & 11 & 16 & 4 & 12 & 1391.6726 & 3 \\
\hline 19 & 3 & 17 & 18 & 3 & 16 & 1391.7603 & -10 \\
\hline 19 & 4 & 16 & 18 & 4 & 15 & 1391.8435 & -7 \\
\hline 19 & 4 & 15 & 18 & 4 & 14 & 1391.8579 & 7 \\
\hline 15 & 5 & 10 & 15 & 4 & 11 & 1391.8957 & 1 \\
\hline 19 & 5 & 14 & 18 & 5 & 13 & 1391.9155 & -5 \\
\hline 19 & 3 & 16 & 18 & 3 & 15 & 1392.0216 & 14 \\
\hline 19 & 6 & 13 & 18 & 6 & 12 & 1392.0292 & -12 \\
\hline 14 & 5 & 9 & 14 & 4 & 10 & 1392.1065 & -6 \\
\hline 21 & 1 & 21 & 20 & 0 & 20 & 1392.2019 & 11 \\
\hline 22 & 1 & 22 & 21 & 1 & 21 & 1392.3067 & -1 \\
\hline 13 & 5 & 8 & 13 & 4 & 9 & 1392.3067 & 3 \\
\hline 19 & 8 & 11 & 18 & 8 & 10 & 1392.3440 & 6 \\
\hline 20 & 2 & 19 & 19 & 2 & 18 & 1392.3727 & -52 \\
\hline 8 & 3 & 6 & 7 & 2 & 5 & 1392.4480 & 3 \\
\hline 8 & 3 & 5 & 7 & 2 & 6 & 1392.4893 & 9 \\
\hline 12 & 5 & 7 & 12 & 4 & 8 & 1392.4933 & 1 \\
\hline 19 & 1 & 18 & 18 & 1 & 17 & 1392.6183 & -16 \\
\hline 11 & 5 & 6 & 11 & 4 & 7 & 1392.6672 & 0 \\
\hline 14 & 2 & 13 & 13 & 1 & 12 & 1392.6912 & -9 \\
\hline 22 & 0 & 22 & 21 & 0 & 21 & 1392.7261 & 7 \\
\hline 19 & 10 & 9 & 18 & 10 & 8 & 1392.7363 & 25 \\
\hline 10 & 5 & 5 & 10 & 4 & 6 & 1392.8283 & 3 \\
\hline 19 & 2 & 17 & 18 & 2 & 16 & 1392.9072 & 37 \\
\hline 9 & 5 & 4 & 9 & 4 & 5 & 1392.9756 & 4 \\
\hline 20 & 3 & 18 & 19 & 3 & 17 & 1393.0860 & -24 \\
\hline 8 & 5 & 3 & 8 & 4 & 4 & 1393.1091 & 4 \\
\hline 4 & 4 & 0 & 3 & 3 & 1 & 1393.1394 & -6 \\
\hline 20 & 4 & 17 & 19 & 4 & 16 & 1393.1727 & -6 \\
\hline 20 & 4 & 16 & 19 & 4 & 15 & 1393.1925 & 0 \\
\hline 7 & 5 & 2 & 7 & 4 & 3 & 1393.2269 & -11 \\
\hline 23 & 1 & 23 & 22 & 1 & 22 & 1393.3003 & 13 \\
\hline 20 & 6 & 14 & 19 & 6 & 13 & 1393.3217 & -6 \\
\hline 6 & 5 & 1 & 6 & 4 & 2 & 1393.3340 & 10 \\
\hline 12 & 2 & 10 & 11 & 1 & 11 & 1393.3976 & 20 \\
\hline 20 & 7 & 13 & 19 & 7 & 12 & 1393.4078 & 14 \\
\hline 5 & 5 & 0 & 5 & 4 & 1 & 1393.4242 & 7 \\
\hline 20 & 3 & 17 & 19 & 3 & 16 & 1393.4331 & 30 \\
\hline 21 & 2 & 20 & 20 & 2 & 19 & 1393.5835 & -60 \\
\hline 20 & 8 & 12 & 19 & 8 & 11 & 1393.5944 & 2 \\
\hline 23 & 0 & 23 & 22 & 0 & 22 & 1393.6846 & 27 \\
\hline 20 & 9 & 11 & 19 & 9 & 10 & 1393.7270 & 19 \\
\hline 15 & 2 & 14 & 14 & 1 & 13 & 1393.7857 & -22 \\
\hline 9 & 3 & 7 & 8 & 2 & 6 & 1393.8780 & 9 \\
\hline 20 & 1 & 19 & 19 & 1 & 18 & 1393.9374 & -22 \\
\hline 9 & 3 & 6 & 8 & 2 & 7 & 1393.9462 & 7 \\
\hline 23 & 1 & 23 & 22 & 0 & 22 & 1394.0057 & 6 \\
\hline 21 & 3 & 19 & 20 & 3 & 18 & 1394.4014 & -24 \\
\hline 20 & 2 & 18 & 19 & 2 & 17 & 1394.4137 & 50 \\
\hline 21 & 4 & 18 & 20 & 4 & 17 & 1394.4951 & 1 \\
\hline 21 & 4 & 17 & 20 & 4 & 16 & 1394.5217 & -11 \\
\hline 21 & 5 & 16 & 20 & 5 & 15 & 1394.5278 & -11 \\
\hline 21 & 6 & 15 & 20 & 6 & 14 & 1394.6030 & -15 \\
\hline 24 & 0 & 24 & 23 & 0 & 23 & 1394.6167 & 17 \\
\hline 5 & 4 & 1 & 4 & 3 & 2 & 1394.6346 & -7 \\
\hline 21 & 7 & 14 & 20 & 7 & 13 & 1394.6771 & 4 \\
\hline 21 & 8 & 13 & 20 & 8 & 12 & 1394.8353 & 7 \\
\hline 16 & 2 & 15 & 15 & 1 & 14 & 1394.8494 & -16 \\
\hline 21 & 3 & 18 & 20 & 3 & 17 & 1394.8494 & 21 \\
\hline 24 & 1 & 24 & 23 & 0 & 23 & 1394.8860 & 2 \\
\hline
\end{tabular}


24 1 23
22
26 22
16 26 11 20 19 16

27

1

1

1

17

18

18

1

28

13
10

18

9
14
14

14
20

20
10

10
15
15

15
23

$$
\begin{array}{rrrrrr}
7 & 17 & 24 & 6 & 18 & 1402.4131 \\
3 & 12 & 14 & 2 & 13 & 1402.6087 \\
5 & 1 & 5 & 4 & 2 & 1402.7374
\end{array}
$$

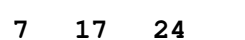$$
\begin{array}{rrrrrr}
5 & 1 & 5 & 4 & 2 & 140 \\
7 & 16 & 23 & 6 & 17 & 140
\end{array}
$$$$
\begin{array}{lllll}
7 & 15 & 22 & 6 & 16 \\
3 & 14 & 15 & 2 & 13
\end{array}
$$$$
3 \quad 14 \quad 15
$$$$
\begin{array}{rrr}
4 & 7 & 10
\end{array}
$$$$
\begin{array}{lll}
7 & 14 & 21
\end{array}
$$$$
\begin{array}{lll}
7 & 13 & 20 \\
7 & 12 & 19
\end{array}
$$$$
3 \quad 12 \quad 19
$$$$
5
$$$$
\begin{array}{lll}
7 & 11 & 18
\end{array}
$$$$
2 \quad 25 \quad 26
$$$$
4
$$$$
7
$$$$
7
$$$$
\begin{array}{rrr}
3 & 14 & 16 \\
7 & 6 & 13
\end{array}
$$$$
\begin{array}{llr}
5 & 3 & 7
\end{array}
$$$$
\begin{array}{lrr}
3 & 16 & 17
\end{array}
$$$$
\begin{array}{rrr}
7 & 5 & 12
\end{array}
$$$$
\begin{array}{rrr}
7 & 4 & 11 \\
2 & 26 & 27
\end{array}
$$$$
4 \quad 10 \quad 27
$$$$
\begin{array}{lll}
4 & 9 & 12 \\
7 & 3 & 10
\end{array}
$$$$
\begin{array}{lll}
7 & 3 & 10
\end{array}
$$

7
7$$
7
$$$$
\begin{array}{lll}
3 & 17 & 18
\end{array}
$$$$
\begin{array}{lll}
3 & 15 & 17
\end{array}
$$$$
\begin{array}{rrr}
5 & 4 & 8 \\
4 & 11 & 13
\end{array}
$$$$
4 \quad 10 \quad 13
$$$$
3 \quad 18 \quad 19
$$$$
\begin{array}{lrr}
5 & 5 & 9
\end{array}
$$$$
\begin{array}{lll}
4 & 12 & 14 \\
4 & 11 & 14
\end{array}
$$$$
\begin{array}{lll}
8 & 15 & 23
\end{array}
$$$$
\begin{array}{rrr}
6 & 0 & 5 \\
8 & 14 & 22
\end{array}
$$$$
\begin{array}{rrr}
5 & 14 & 22 \\
8 & 13 & 10 \\
4 & 13 & 15
\end{array}
$$$$
\begin{array}{lll}
4 & 13 & 15 \\
4 & 12 & 15
\end{array}
$$$$
4 \quad 12 \quad 15
$$$$
\begin{array}{lll}
8 & 12 & 20 \\
8 & 11 & 19
\end{array}
$$$$
\begin{array}{rrr}
6 & 1 & 6 \\
8 & 10 & 18 \\
8 & 9 & 17
\end{array}
$$$$
\begin{array}{rrr}
8 & 10 & 18 \\
5 & 9 & 17 \\
8 & 7 & 11 \\
4 & 8 & 16 \\
8 & 13 & 16
\end{array}
$$$$
\begin{array}{rrr}
4 & 13 & 16 \\
8 & 7 & 15
\end{array}
$$$$
8 \quad 6 \quad 14
$$$$
\begin{array}{rrr}
8 & 5 & 13 \\
6 & 2 & 7 \\
8 & 4 & 12
\end{array}
$$$$
23
$$

$\begin{array}{rrr}1402.6087 & 8 & 4 \\ 1402.7374 & -1 & 4\end{array}$ $1402.7498 \quad 15 \quad 4$ $1403.0755 \quad 12 \quad 4$ $1403.2413 \quad 5 \quad 4$ $1403.2529 \quad 16 \quad 4$ 


\begin{tabular}{|c|c|c|c|c|c|c|c|c|c|c|c|c|}
\hline 11 & 1417.1309 & 7 & 4 & 16 & 10 & 6 & 15 & 9 & 7 & 1449.8090 & -38 & 10 \\
\hline 10 & 1417.4634 & -1 & 4 & 17 & 10 & 7 & 16 & 9 & 8 & 1451.0338 & -27 & 4 \\
\hline 1 & 1417.5742 & 7 & 4 & 18 & 10 & 8 & 17 & 9 & 9 & 1452.2467 & -19 & 4 \\
\hline 7 & 1417.9161 & -2 & 4 & 19 & 10 & 9 & 18 & 9 & 10 & 1453.4510 & 5 & \\
\hline
\end{tabular}

$v_{5}$ Band

$\begin{array}{rrrrrr}33 & 10 & 23 & 34 & 10 & 24 \\ 33 & 9 & 24 & 34 & 9 & 25 \\ 32 & 10 & 22 & 33 & 10 & 23 \\ 32 & 9 & 23 & 33 & 9 & 24 \\ 32 & 6 & 26 & 33 & 6 & 27 \\ 32 & 7 & 25 & 33 & 7 & 26 \\ 32 & 4 & 28 & 33 & 4 & 29 \\ 32 & 4 & 29 & 33 & 4 & 30 \\ 31 & 10 & 21 & 32 & 10 & 22 \\ 31 & 9 & 22 & 32 & 9 & 23 \\ 31 & 6 & 25 & 32 & 6 & 26 \\ 31 & 7 & 24 & 32 & 7 & 25 \\ 31 & 4 & 27 & 32 & 4 & 28 \\ 31 & 4 & 28 & 32 & 4 & 29 \\ 31 & 6 & 26 & 32 & 6 & 27\end{array}$

$\begin{array}{llllll}30 & 11 & 19 & 31 & 11 & 20\end{array}$

$\begin{array}{llllll}30 & 10 & 20 & 31 & 10 & 21\end{array}$

$\begin{array}{llllll}30 & 3 & 27 & 31 & 3 & 28 \\ 30 & 9 & 21 & 31 & 9 & 22\end{array}$

$\begin{array}{llllll}30 & 6 & 24 & 31 & 6 & 25\end{array}$

$\begin{array}{llllll}30 & 7 & 23 & 31 & 7 & 24\end{array}$

$\begin{array}{llllll}30 & 4 & 26 & 31 & 4 & 27\end{array}$

$\begin{array}{llllll}30 & 5 & 26 & 31 & 5 & 27 \\ 30 & 5 & 25 & 31 & 5 & 26\end{array}$

$\begin{array}{llllll}30 & 4 & 27 & 31 & 4 & 28\end{array}$

$\begin{array}{llllll}30 & 3 & 28 & 31 & 3 & 29\end{array}$

$\begin{array}{llllll}30 & 6 & 25 & 31 & 6 & 26\end{array}$

$\begin{array}{llllll}29 & 11 & 18 & 30 & 11 & 19 \\ 29 & 10 & 19 & 30 & 10 & 20\end{array}$

$\begin{array}{rrrrrr}29 & 3 & 26 & 30 & 3 & 27\end{array}$

$\begin{array}{llllll}29 & 9 & 20 & 30 & 9 & 21 \\ 29 & 6 & 23 & 30 & 6 & 24\end{array}$

$\begin{array}{llllll}29 & 7 & 22 & 30 & 7 & 23\end{array}$

$\begin{array}{llllll}29 & 8 & 21 & 30 & 8 & 22\end{array}$

$\begin{array}{llllll}29 & 4 & 25 & 30 & 4 & 26\end{array}$

$\begin{array}{llllll}29 & 5 & 25 & 30 & 5 & 26 \\ 29 & 5 & 24 & 30 & 5 & 25\end{array}$

$\begin{array}{llllll}29 & 4 & 26 & 30 & 4 & 27\end{array}$

$\begin{array}{llllll}29 & 6 & 24 & 30 & 6 & 25 \\ 29 & 3 & 27 & 30 & 3 & 28\end{array}$

$\begin{array}{llllll}28 & 11 & 17 & 29 & 11 & 18\end{array}$

$\begin{array}{rrrrrr}28 & 10 & 18 & 29 & 10 & 19 \\ 28 & 9 & 19 & 29 & 9 & 20\end{array}$

$\begin{array}{llllll}28 & 3 & 25 & 29 & 3 & 26\end{array}$

$\begin{array}{llllll}28 & 8 & 20 & 29 & 8 & 21\end{array}$

$\begin{array}{llllll}28 & 7 & 21 & 29 & 7 & 22 \\ 28 & 4 & 24 & 29 & 4 & 25\end{array}$

$\begin{array}{llllll}28 & 4 & 24 & 29 & 4 & 25 \\ 28 & 5 & 24 & 29 & 5 & 25\end{array}$

$\begin{array}{llllll}28 & 5 & 23 & 29 & 5 & 24 \\ 28 & 4 & 25 & 29 & 4 & 26\end{array}$

28
28

28
28

28

27
27

27

27

26
27

27

27

27
27

27
27

27

$\begin{array}{llllrl}26 & 11 & 15 & 27 & 11 & 16\end{array}$

$\begin{array}{llllll}26 & 10 & 16 & 27 & 10 & 17\end{array}$

$\begin{array}{rrrrrr}26 & 9 & 17 & 27 & 9 & 18 \\ 26 & 8 & 18 & 27 & 8 & 19\end{array}$
$991.1775 \quad-34 \quad 4$

$991.5613-61 \quad 4$

$993.2630 \quad-26 \quad 4$

$993.6487 \quad-44 \quad 4$

$993.9142 \quad-14 \quad 4$

$\begin{array}{lll}993.9576 & -7 & 10\end{array}$

$993.9576 \quad 18 \quad 10$

$994.1197 \quad 134$

$995.3411 \quad-9 \quad 4$

$995.7270-27 \quad 4$

$995.9990 \quad-23 \quad 4$

$996.0564 \quad 1 \quad 4$

$996.1249 \quad 11 \quad 4$

$996.2546 \quad 12 \quad 4$

$996.8248 \quad 101 \quad 10$

$996.8860 \quad 17 \quad 4$

$997.4092 \quad-5 \quad 4$

$997.5668 \quad 11 \quad 4$

$997.7950 \quad-17 \quad 4$

$998.0479 \quad-27 \quad 4$

$998.1421 \quad 0 \quad 4$

$998.2708 \quad 8 \quad 4$

$998.3381 \quad 1 \quad 4$

$998.3697 \quad 2 \quad 10$

$998.3728 \quad 3 \quad 10$

$998.5884 \quad 17 \quad 4$

$998.7130 \quad 56 \quad 4$

$998.9435 \quad 19 \quad 4$

$999.4685 \quad 2 \quad 4$

$999.7872 \quad 7 \quad 4$

$999.8526-11 \quad 4$

$1000.0278-42 \quad 4$

$1000.2150-3 \quad 4$

$1000.2310 \quad-3 \quad 4$

$1000.3958 \quad 7 \quad 4$

$1000.4359 \quad 2 \quad 4$

$\begin{array}{lll}1000.4639 & 3 & 4\end{array}$

$1000.4761 \quad 8 \quad 4$

$\begin{array}{lll}1000.6544 & 23 & 4 \\ 1000.6603 & 15 & 4\end{array}$

$1000.9938 \quad 21 \quad 4$

$1001.5180 \quad 7 \quad 4$

$1001.8996 \quad-4 \quad 4$

$1001.9810 \quad 6 \quad 4$

$\begin{array}{rrr}1002.2412 & 6 & 4\end{array}$

$1002.4999 \quad 5 \quad 4$

$1002.5166 \quad 0 \quad 4$

$1002.5411 \quad 1 \quad 4$

$1002.5620 \quad 4 \quad 4$

$1002.6317 \quad 11 \quad 4$

$1002.7199 \quad 10 \quad 4$

$1002.9977-48 \quad 4$

$1003.0357 \quad 16 \quad 4$

$1003.5581 \quad 17 \quad 4$

$1003.9354 \quad 0 \quad 4$

$1004.1477 \quad 5 \quad 4$

$1004.2492 \quad 10 \quad 4$

$1004.2840 \quad 9 \quad 4$

$\begin{array}{rrr}1004.3219 & -4 & 4 \\ 1004.5823 & -10 & 10\end{array}$

$1004.6016 \quad-1 \quad 4$

$1004.6254 \quad 0 \quad 4$

$1004.6316 \quad 5 \quad 4$

$1004.7606 \quad-17 \quad 4$

$1004.7673 \quad 9 \quad 4$

$1005.0704 \quad 20 \quad 4$

$1005.5863 \quad 12 \quad 4$

$\begin{array}{rrr}1005.9597 & 4 & 4\end{array}$ 


\begin{tabular}{|c|c|c|c|c|c|c|c|}
\hline 26 & 3 & 23 & 27 & 3 & 24 & 1006.2869 & 3 \\
\hline 25 & 12 & 13 & 26 & 12 & 14 & 1006.3184 & 3 \\
\hline 26 & 7 & 19 & 27 & 7 & 20 & 1006.3547 & -6 \\
\hline 26 & 5 & 22 & 27 & 5 & 23 & 1006.6261 & -15 \\
\hline 26 & 6 & 21 & 27 & 6 & 22 & 1006.6261 & 16 \\
\hline 26 & 4 & 22 & 27 & 4 & 23 & 1006.6465 & -9 \\
\hline 26 & 5 & 21 & 27 & 5 & 22 & 1006.6465 & 9 \\
\hline 26 & 6 & 20 & 27 & 6 & 21 & 1006.6758 & -9 \\
\hline 26 & 4 & 23 & 27 & 4 & 24 & 1006.6837 & 0 \\
\hline 26 & 3 & 24 & 27 & 3 & 25 & 1006.8013 & 6 \\
\hline 25 & 11 & 14 & 26 & 11 & 15 & 1007.0957 & 16 \\
\hline 25 & 2 & 23 & 26 & 2 & 24 & 1007.5527 & 1 \\
\hline 25 & 10 & 15 & 26 & 10 & 16 & 1007.6051 & 20 \\
\hline 25 & 9 & 16 & 26 & 9 & 17 & 1007.9719 & 6 \\
\hline 25 & 8 & 17 & 26 & 8 & 18 & 1008.2473 & 13 \\
\hline 25 & 1 & 24 & 26 & 1 & 25 & 1008.2645 & 3 \\
\hline 24 & 12 & 12 & 25 & 12 & 13 & 1008.3480 & -2 \\
\hline 25 & 7 & 18 & 26 & 7 & 19 & 1008.3736 & -7 \\
\hline 25 & 3 & 22 & 26 & 3 & 23 & 1008.3990 & 1 \\
\hline 25 & 6 & 20 & 26 & 6 & 21 & 1008.6201 & -4 \\
\hline 25 & 6 & 19 & 26 & 6 & 20 & 1008.6407 & -15 \\
\hline 25 & 5 & 21 & 26 & 5 & 22 & 1008.6573 & -3 \\
\hline 25 & 5 & 20 & 26 & 5 & 21 & 1008.6724 & -3 \\
\hline 25 & 4 & 21 & 26 & 4 & 22 & 1008.6918 & 0 \\
\hline 25 & 3 & 23 & 26 & 3 & 24 & 1008.8217 & 3 \\
\hline 24 & 11 & 13 & 25 & 11 & 14 & 1009.1124 & 16 \\
\hline 25 & 2 & 24 & 26 & 2 & 25 & 1009.5382 & 9 \\
\hline 24 & 10 & 14 & 25 & 10 & 15 & 1009.6112 & 14 \\
\hline 24 & 2 & 22 & 25 & 2 & 23 & 1009.6749 & 1 \\
\hline 24 & 9 & 15 & 25 & 9 & 16 & 1009.9717 & 8 \\
\hline 24 & 1 & 23 & 25 & 1 & 24 & 1010.2300 & 6 \\
\hline 24 & 8 & 16 & 25 & 8 & 17 & 1010.2330 & 11 \\
\hline 23 & 12 & 11 & 24 & 12 & 12 & 1010.3704 & -26 \\
\hline 24 & 7 & 17 & 25 & 7 & 18 & 1010.3780 & -9 \\
\hline 24 & 3 & 21 & 25 & 3 & 22 & 1010.4843 & 1 \\
\hline 24 & 6 & 19 & 25 & 6 & 20 & 1010.6081 & -7 \\
\hline 24 & 6 & 18 & 25 & 6 & 19 & 1010.6177 & -7 \\
\hline 24 & 5 & 20 & 25 & 5 & 21 & 1010.6700 & -5 \\
\hline 24 & 5 & 19 & 25 & 5 & 20 & 1010.6827 & -4 \\
\hline 24 & 4 & 20 & 25 & 4 & 21 & 1010.7168 & -1 \\
\hline 24 & 4 & 21 & 25 & 4 & 22 & 1010.7370 & -2 \\
\hline 24 & 3 & 22 & 25 & 3 & 23 & 1010.8280 & 3 \\
\hline 23 & 11 & 12 & 24 & 11 & 13 & 1011.1194 & 13 \\
\hline 24 & 2 & 23 & 25 & 2 & 24 & 1011.4682 & 5 \\
\hline 23 & 10 & 13 & 24 & 10 & 14 & 1011.6061 & 13 \\
\hline 23 & 2 & 21 & 24 & 2 & 22 & 1011.7813 & 0 \\
\hline 23 & 9 & 14 & 24 & 9 & 15 & 1011.9586 & 8 \\
\hline 23 & 1 & 22 & 24 & 1 & 23 & 1012.1925 & 2 \\
\hline 23 & 8 & 15 & 24 & 8 & 16 & 1012.2083 & 11 \\
\hline 23 & 7 & 16 & 24 & 7 & 17 & 1012.3676 & -11 \\
\hline 22 & 12 & 10 & 23 & 12 & 11 & 1012.3914 & -8 \\
\hline 23 & 3 & 20 & 24 & 3 & 21 & 1012.5431 & 0 \\
\hline 23 & 6 & 18 & 24 & 6 & 19 & 1012.5859 & -6 \\
\hline 23 & 6 & 17 & 24 & 6 & 18 & 1012.5898 & -11 \\
\hline 23 & 5 & 19 & 24 & 5 & 20 & 1012.6659 & -4 \\
\hline 23 & 5 & 18 & 24 & 5 & 19 & 1012.6760 & -5 \\
\hline 23 & 4 & 19 & 24 & 4 & 20 & 1012.7228 & -2 \\
\hline 23 & 4 & 20 & 24 & 4 & 21 & 1012.7376 & -2 \\
\hline 23 & 3 & 21 & 24 & 3 & 22 & 1012.8193 & -1 \\
\hline 22 & 11 & 11 & 23 & 11 & 12 & 1013.1164 & 10 \\
\hline 23 & 2 & 22 & 24 & 2 & 23 & 1013.3889 & 3 \\
\hline 22 & 10 & 12 & 23 & 10 & 13 & 1013.5889 & 13 \\
\hline 22 & 2 & 20 & 23 & 2 & 21 & 1013.8706 & 0 \\
\hline 22 & 9 & 13 & 23 & 9 & 14 & 1013.9323 & 8 \\
\hline 22 & 1 & 21 & 23 & 1 & 22 & 1014.1516 & -1 \\
\hline 22 & 8 & 14 & 23 & 8 & 15 & 1014.1715 & 9 \\
\hline 22 & 7 & 15 & 23 & 7 & 16 & 1014.3424 & -10 \\
\hline 21 & 12 & 9 & 22 & 12 & 10 & 1014.4041 & -13 \\
\hline 22 & 6 & 16 & 23 & 6 & 17 & 1014.5520 & -18 \\
\hline 22 & 3 & 19 & 23 & 3 & 20 & 1014.5762 & 0 \\
\hline 22 & 5 & 18 & 23 & 5 & 19 & 1014.6446 & -4 \\
\hline 22 & 5 & 17 & 23 & 5 & 18 & 1014.6526 & -5 \\
\hline 22 & 4 & 18 & 23 & 4 & 19 & 1014.7098 & -4 \\
\hline 22 & 4 & 19 & 23 & 4 & 20 & 1014.7210 & 1 \\
\hline 22 & 3 & 20 & 23 & 3 & 21 & 1014.7957 & -1 \\
\hline 21 & 11 & 10 & 22 & 11 & 11 & 1015.1032 & 11 \\
\hline 22 & 2 & 21 & 23 & 2 & 22 & 1015.2998 & \\
\hline
\end{tabular}




\begin{tabular}{|c|c|c|c|c|c|c|c|}
\hline 17 & 4 & 13 & 18 & 4 & 14 & 1024.3687 & 4 \\
\hline 17 & 3 & 14 & 18 & 3 & 15 & 1024.3777 & -3 \\
\hline 17 & 3 & 15 & 18 & 3 & 16 & 1024.4344 & -3 \\
\hline 17 & 2 & 16 & 18 & 2 & 17 & 1024.6896 & -4 \\
\hline 16 & 11 & 5 & 17 & 11 & 6 & 1024.8569 & 5 \\
\hline 16 & 10 & 6 & 17 & 10 & 7 & 1025.2018 & 6 \\
\hline 17 & 0 & 17 & 18 & 0 & 18 & 1025.3130 & -3 \\
\hline 16 & 9 & 7 & 17 & 9 & 8 & 1025.4727 & 5 \\
\hline 16 & 8 & 8 & 17 & 8 & 9 & 1025.6488 & -7 \\
\hline 16 & 1 & 15 & 17 & 1 & 16 & 1025.7541 & 3 \\
\hline 17 & 1 & 17 & 18 & 1 & 18 & 1025.7584 & 1 \\
\hline 16 & 7 & 9 & 17 & 7 & 10 & 1025.8586 & -9 \\
\hline 16 & 2 & 14 & 17 & 2 & 15 & 1025.9655 & -2 \\
\hline 16 & 6 & 10 & 17 & 6 & 11 & 1026.0297 & -5 \\
\hline 16 & 5 & 11 & 17 & 5 & 12 & 1026.1529 & -12 \\
\hline 16 & 4 & 12 & 17 & 4 & 13 & 1026.2451 & 1 \\
\hline 16 & 3 & 13 & 17 & 3 & 14 & 1026.2700 & -4 \\
\hline 16 & 3 & 14 & 17 & 3 & 15 & 1026.3112 & -3 \\
\hline 15 & 12 & 3 & 16 & 12 & 4 & 1026.3286 & -19 \\
\hline 16 & 2 & 15 & 17 & 2 & 16 & 1026.5313 & -3 \\
\hline 15 & 11 & 4 & 16 & 11 & 5 & 1026.7668 & 0 \\
\hline 16 & 0 & 16 & 17 & 0 & 17 & 1027.0588 & -4 \\
\hline 15 & 10 & 5 & 16 & 10 & 6 & 1027.0853 & 5 \\
\hline 15 & 9 & 6 & 16 & 9 & 7 & 1027.3419 & 3 \\
\hline 15 & 8 & 7 & 16 & 8 & 8 & 1027.4961 & -10 \\
\hline 16 & 1 & 16 & 17 & 1 & 17 & 1027.5030 & -5 \\
\hline 15 & 1 & 14 & 16 & 1 & 15 & 1027.6494 & 0 \\
\hline 15 & 7 & 8 & 16 & 7 & 9 & 1027.7200 & -8 \\
\hline 15 & 6 & 9 & 16 & 6 & 10 & 1027.8859 & -5 \\
\hline 15 & 2 & 13 & 16 & 2 & 14 & 1027.8969 & -2 \\
\hline 15 & 5 & 10 & 16 & 5 & 11 & 1028.0095 & -10 \\
\hline 15 & 4 & 11 & 16 & 4 & 12 & 1028.1035 & -1 \\
\hline 15 & 3 & 12 & 16 & 3 & 13 & 1028.1411 & -3 \\
\hline 15 & 3 & 13 & 16 & 3 & 14 & 1028.1704 & -3 \\
\hline 14 & 12 & 2 & 15 & 12 & 3 & 1028.2855 & -13 \\
\hline 15 & 2 & 14 & 16 & 2 & 15 & 1028.3598 & -3 \\
\hline 14 & 11 & 3 & 15 & 11 & 4 & 1028.6623 & 4 \\
\hline 15 & 0 & 15 & 16 & 0 & 16 & 1028.8011 & -4 \\
\hline 14 & 10 & 4 & 15 & 10 & 5 & 1028.9525 & 5 \\
\hline 14 & 9 & 5 & 15 & 9 & 6 & 1029.1946 & 3 \\
\hline 15 & 1 & 15 & 16 & 1 & 16 & 1029.2392 & -5 \\
\hline 14 & 8 & 6 & 15 & 8 & 7 & 1029.4573 & 11 \\
\hline 14 & 1 & 13 & 15 & 1 & 14 & 1029.5310 & 1 \\
\hline 14 & 7 & 7 & 15 & 7 & 8 & 1029.5642 & -7 \\
\hline 14 & 6 & 8 & 15 & 6 & 9 & 1029.7251 & -4 \\
\hline 14 & 2 & 12 & 15 & 2 & 13 & 1029.8022 & -1 \\
\hline 14 & 5 & 9 & 15 & 5 & 10 & 1029.8486 & -8 \\
\hline 14 & 4 & 10 & 15 & 4 & 11 & 1029.9438 & -1 \\
\hline 14 & 3 & 11 & 15 & 3 & 12 & 1029.9914 & -3 \\
\hline 14 & 3 & 12 & 15 & 3 & 13 & 1030.0118 & -3 \\
\hline 14 & 2 & 13 & 15 & 2 & 14 & 1030.1746 & -3 \\
\hline 13 & 12 & 1 & 14 & 12 & 2 & 1030.2311 & -11 \\
\hline 14 & 0 & 14 & 15 & 0 & 15 & 1030.5398 & -2 \\
\hline 13 & 10 & 3 & 14 & 10 & 4 & 1030.8028 & 4 \\
\hline 14 & 1 & 14 & 15 & 1 & 15 & 1030.9660 & -5 \\
\hline 13 & 9 & 4 & 14 & 9 & 5 & 1031.0304 & 3 \\
\hline 13 & 8 & 5 & 14 & 8 & 6 & 1031.2512 & 7 \\
\hline 13 & 7 & 6 & 14 & 7 & 7 & 1031.3909 & -6 \\
\hline 13 & 1 & 12 & 14 & 1 & 13 & 1031.3975 & 2 \\
\hline 13 & 6 & 7 & 14 & 6 & 8 & 1031.5470 & -3 \\
\hline 13 & 5 & 8 & 14 & 5 & 9 & 1031.6701 & -8 \\
\hline 13 & 2 & 11 & 14 & 2 & 12 & 1031.6811 & -1 \\
\hline 13 & 4 & 9 & 14 & 4 & 10 & 1031.7660 & -2 \\
\hline 13 & 3 & 10 & 14 & 3 & 11 & 1031.8215 & -2 \\
\hline 13 & 3 & 11 & 14 & 3 & 12 & 1031.8353 & -2 \\
\hline 13 & 2 & 12 & 14 & 2 & 13 & 1031.9753 & -3 \\
\hline 13 & 0 & 13 & 14 & 0 & 14 & 1032.2740 & -2 \\
\hline 12 & 11 & 1 & 13 & 11 & 2 & 1032.4033 & 3 \\
\hline 12 & 10 & 2 & 13 & 10 & 3 & 1032.6359 & 5 \\
\hline 13 & 1 & 13 & 14 & 1 & 14 & 1032.6833 & -4 \\
\hline 12 & 9 & 3 & 13 & 9 & 4 & 1032.8492 & 6 \\
\hline 12 & 8 & 4 & 13 & 8 & 5 & 1033.0484 & 4 \\
\hline 12 & 7 & 5 & 13 & 7 & 6 & 1033.1999 & -5 \\
\hline 12 & 1 & 11 & 13 & 1 & 12 & 1033.2478 & 1 \\
\hline 12 & 6 & 6 & 13 & 6 & 7 & 1033.3514 & -3 \\
\hline 12 & 5 & 7 & 13 & 5 & 8 & 1033.4725 & 9 \\
\hline 12 & 2 & 10 & 13 & 2 & 11 & 1033.5337 & -1 \\
\hline
\end{tabular}

\begin{tabular}{|c|c|c|c|c|c|c|c|c|}
\hline 12 & $=$ & 8 & 13 & 4 & 9 & 1033. & 5701 & -2 \\
\hline 12 & 3 & 9 & 13 & 3 & 10 & 1033. & 6315 & -2 \\
\hline 12 & 3 & 10 & 13 & 3 & 11 & 1033. & 6406 & -2 \\
\hline & 2 & 11 & 13 & 2 & 12 & 1033 & 7615 & -2 \\
\hline & 0 & 12 & 13 & 0 & 13 & & 0034 & -2 \\
\hline 1 & 11 & 0 & 12 & 11 & 1 & 1034 & 2478 & 5 \\
\hline 1 & 1 & 12 & 13 & 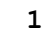 & 13 & 1034 & 3905 & -5 \\
\hline 11 & 10 & 1 & 12 & 10 & 2 & 1034 & 4510 & 5 \\
\hline 11 & 9 & 2 & 12 & 9 & 3 & 1034 & 6496 & 3 \\
\hline 11 & 8 & 3 & 12 & 8 & 4 & 1034 & 8360 & 3 \\
\hline 11 & 7 & 4 & 12 & 7 & 5 & 1034 & 9910 & -3 \\
\hline 1 & 1 & 10 & 12 & 1 & 11 & 1035. & 0812 & 2 \\
\hline 11 & 6 & 5 & 12 & & 6 & 1035. & 1382 & -2 \\
\hline 11 & 5 & 6 & 12 & 5 & 7 & 1035 . & 2589 & -2 \\
\hline 11 & 4 & 7 & 12 & 4 & 8 & 1035. & 3563 & 0 \\
\hline 11 & 2 & 9 & 12 & 2 & 10 & 1035. & 3611 & 7 \\
\hline 11 & 3 & 8 & 12 & 3 & 9 & 1035. & 4219 & -3 \\
\hline 11 & 3 & 9 & 12 & 3 & 10 & 1035. & 4280 & 0 \\
\hline 11 & 2 & 10 & 12 & ? & 11 & 1035. & 5327 & -1 \\
\hline 11 & 0 & 11 & 12 & & 12 & 1035. & 7271 & -1 \\
\hline 11 & 1 & 11 & 12 & 1 & 12 & 1036. & & -4 \\
\hline 10 & 10 & 0 & 11 & 10 & 1 & 1036. & 2474 & 4 \\
\hline 10 & 9 & 1 & 11 & 9 & 2 & 1036. & 4322 & 3 \\
\hline 10 & 8 & 2 & 11 & 8 & 3 & 1036. & 6088 & 1 \\
\hline 10 & 7 & 3 & 11 & 7 & 4 & 1036 & 7639 & -2 \\
\hline 10 & 1 & 9 & 11 & 1 & 10 & 1036. & 8969 & 3 \\
\hline 10 & 6 & 4 & 11 & 0 & 5 & 1036 . & 9072 & -1 \\
\hline 10 & 5 & 5 & 11 & 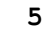 & 6 & & & -2 \\
\hline 10 & 4 & 6 & 11 & 4 & 7 & 1037. & 1241 & -1 \\
\hline 10 & 2 & 8 & 11 & 2 & 9 & 1037. & 1616 & 0 \\
\hline 10 & 3 & 7 & 11 & 3 & 8 & 1037. & 1946 & 13 \\
\hline 10 & 2 & 9 & 11 & 2 & 10 & 1037. & 2886 & -1 \\
\hline 10 & 0 & 10 & 11 & 0 & 11 & 1037. & 4439 & -1 \\
\hline 10 & 1 & 10 & 11 & 1 & 11 & & 77 & -3 \\
\hline 9 & 9 & 0 & 10 & 9 & 1 & 1038. & 1965 & 4 \\
\hline 9 & 8 & 1 & 10 & 8 & 2 & 1038. & 3651 & 2 \\
\hline 9 & 7 & 2 & 10 & 7 & 3 & 1038. & 5184 & -1 \\
\hline 9 & 6 & 3 & 10 & 6 & 4 & 1038 & 6583 & -1 \\
\hline 9 & 1 & 8 & 10 & 1 & 9 & 1038 & 6940 & 2 \\
\hline 9 & 5 & 4 & 10 & & 5 & 1038 & 7769 & -1 \\
\hline 9 & 4 & 5 & 10 & 4 & 6 & 1038. & 87 & -1 \\
\hline 9 & 2 & 7 & 10 & & 8 & 1038. & 93 & 1 \\
\hline 9 & 3 & 6 & 10 & 3 & 7 & 1038. & 9463 & 10 \\
\hline 9 & 2 & 8 & 10 & 2 & 9 & 1039. & 0288 & 1 \\
\hline 9 & 0 & 9 & 10 & 0 & 10 & 1039. & 1528 & 1 \\
\hline 9 & 1 & 9 & 10 & 1 & 10 & 1039. & 4495 & -1 \\
\hline 8 & 8 & 0 & 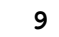 & 8 & 1 & 1040. & 10 & 0 \\
\hline 8 & 7 & 1 & 9 & & 2 & 1040. & 25 & 0 \\
\hline 8 & 6 & 2 & 9 & 6 & 3 & 1040. & 3913 & -1 \\
\hline 8 & 1 & 7 & 9 & 1 & 8 & 1040 . & 4721 & 3 \\
\hline 8 & 5 & 3 & 9 & 5 & 4 & 1040 & 5088 & 0 \\
\hline 8 & 4 & 4 & 9 & 4 & 5 & 1040. & 60 & 0 \\
\hline 8 & & 5 & 9 & 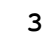 & 6 & 1040. & 67 & 6 \\
\hline 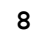 & & 6 & 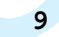 & ? & 7 & 1040. & 69 & 1 \\
\hline 8 & 2 & 7 & 9 & 2 & 8 & 1040. & $7 !$ & 1 \\
\hline 8 & 0 & 8 & 9 & 0 & 9 & 1040 & 8524 & 1 \\
\hline 8 & 1 & 8 & 9 & 1 & 9 & 1041. & 1137 & 0 \\
\hline & 7 & 0 & 0 & 7 & 1 & & & 0 \\
\hline 7 & 6 & 1 & & 6 & 2 & & & 0 \\
\hline 7 & & 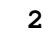 & & I & 3 & 1042 . & 22 & 0 \\
\hline 7 & & 6 & & 1 & 7 & 1042 & 23 & 4 \\
\hline 7 & & 3 & 8 & 4 & 4 & 1042 & 3 & 0 \\
\hline 7 & 3 & 4 & 8 & 3 & 5 & 1042. & 3 & 4 \\
\hline 7 & 2 & 5 & 0 & 2 & 6 & & & 2 \\
\hline 7 & 2 & 6 & & 2 & 7 & & & 2 \\
\hline 7 & $c$ & 7 & & 0 & 8 & & & 2 \\
\hline 7 & & 7 & & & 8 & 1042 . & 76 & 0 \\
\hline 6 & 6 & 0 & & & 1 & 1043. & 80 & 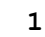 \\
\hline 6 & 5 & 1 & 7 & 5 & 2 & 1043. & 9 & 1 \\
\hline 6 & 1 & 5 & 7 & 1 & 6 & & 9 & 4 \\
\hline 6 & 4 & 2 & 7 & 4 & 3 & & & 1 \\
\hline 6 & 3 & 3 & 7 & 3 & 4 & 1044 & $0 \xi$ & 4 \\
\hline 6 & 2 & 4 & 7 & 2 & 5 & 1044 & 1257 & 2 \\
\hline 6 & & 5 & & & 6 & 1044 & 1516 & 2 \\
\hline 6 & & 6 & 7 & 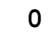 & 7 & 1044. & 21 & 3 \\
\hline 6 & & 6 & 1 & & 1 & 1044. & 40 & 1 \\
\hline 5 & & & 6 & & & 1045. & 5957 & 0 \\
\hline
\end{tabular}




\begin{tabular}{|c|c|c|c|c|c|c|c|c|}
\hline 18 & 11 & 8 & 18 & 11 & 7 & 1050.6889 & 4 & 4 \\
\hline 2 & 1 & 1 & 3 & 1 & 2 & 1050.7095 & 8 & 4 \\
\hline 19 & 10 & 10 & 19 & 10 & 9 & 1050.7229 & 9 & 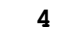 \\
\hline 2 & 2 & 0 & 3 & 2 & 1 & 1050.7429 & 11 & \\
\hline 2 & 0 & 2 & 3 & 0 & 3 & 1050.7889 & 4 & \\
\hline 2 & 1 & 2 & 3 & 1 & 3 & 1050.8442 & 4 & \\
\hline 20 & 8 & 13 & 20 & 8 & 12 & 1050.9001 & 2 & \\
\hline 21 & 6 & 16 & 21 & 6 & 15 & 1050.9240 & -4 & \\
\hline 16 & 12 & 5 & 16 & 12 & 4 & 1050.9300 & -19 & \\
\hline 19 & 9 & 11 & 19 & 9 & 10 & 1051.0433 & 9 & \\
\hline 17 & 11 & 7 & 17 & 11 & 6 & 1051.0686 & 4 & \\
\hline 18 & 10 & 9 & 18 & 10 & 8 & 1051.0938 & 13 & \\
\hline 20 & 7 & 14 & 20 & 7 & 13 & 1051.0979 & 0 & 10 \\
\hline 21 & 4 & 18 & 21 & 4 & 17 & 1051.1290 & -1 & 4 \\
\hline 21 & 4 & 17 & 21 & 4 & 18 & 1051.1396 & 0 & 10 \\
\hline 19 & 8 & 12 & 19 & 8 & 11 & 1051.2632 & 0 & \\
\hline 20 & 6 & 15 & 20 & 6 & 14 & 1051.3009 & -4 & \\
\hline 15 & 12 & 4 & 15 & 12 & 3 & 1051.3370 & -18 & \\
\hline 18 & 9 & 10 & 18 & 9 & 9 & 1051.4004 & 7 & \\
\hline 20 & 5 & 16 & 20 & 5 & 15 & 1051.4221 & -8 & \\
\hline 20 & 5 & 15 & 20 & 5 & 16 & 1051.4275 & -5 & \\
\hline 16 & 11 & 6 & 16 & 11 & 5 & 1051.4337 & 2 & \\
\hline 17 & 10 & 8 & 17 & 10 & 7 & 1051.4484 & 8 & \\
\hline 19 & 7 & 13 & 19 & 7 & 12 & 1051.4648 & -11 & \\
\hline 20 & 4 & 17 & 20 & 4 & 16 & 1051.5167 & 3 & \\
\hline 20 & 4 & 16 & 20 & 4 & 17 & 1051.5233 & -3 & \\
\hline 18 & 8 & 11 & 18 & 8 & 10 & 1051.6100 & -3 & 4 \\
\hline 19 & 6 & 14 & 19 & 6 & 13 & 1051.6617 & -6 & \\
\hline 14 & 12 & 3 & 14 & 12 & 2 & 1051.7337 & -13 & \\
\hline 17 & 9 & 9 & 17 & 9 & 8 & 1051.7414 & 4 & \\
\hline 15 & 11 & 5 & 15 & 11 & 4 & 1051.7833 & -4 & 10 \\
\hline 16 & 10 & 7 & 16 & 10 & 6 & 1051.7862 & -7 & \\
\hline 18 & 7 & 12 & 18 & 7 & 11 & 1051.8159 & -11 & \\
\hline 19 & 3 & 17 & 19 & 3 & 16 & 1051.8446 & -6 & 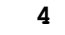 \\
\hline 19 & 4 & 16 & 19 & 4 & 15 & 1051.8843 & -5 & \\
\hline 19 & 4 & 15 & 19 & 4 & 16 & 1051.8893 & -3 & \\
\hline 17 & 8 & 10 & 17 & 8 & 9 & 1051.9396 & -6 & \\
\hline 18 & 6 & 13 & 18 & 6 & 12 & 1052.0061 & -8 & \\
\hline 16 & 9 & 8 & 16 & 9 & 7 & 1052.0663 & 4 & \\
\hline 15 & 10 & 6 & 15 & 10 & 5 & 1052.1103 & 6 & \\
\hline 13 & 12 & 2 & 13 & 12 & 1 & 1052.1185 & -11 & \\
\hline 14 & 11 & 4 & 14 & 11 & 3 & 1052.1185 & 4 & \\
\hline 18 & 5 & 14 & 18 & 5 & 13 & 1052.1359 & 8 & \\
\hline 17 & 7 & 11 & 17 & 7 & 10 & 1052.1499 & -11 & \\
\hline 18 & 3 & 16 & 18 & 3 & 15 & 1052.2242 & -5 & \\
\hline 18 & 4 & 15 & 18 & 4 & 14 & 1052.2348 & 5 & 10 \\
\hline 16 & 8 & 9 & 16 & 8 & 8 & 1052.2498 & -8 & \\
\hline 17 & 6 & 12 & 17 & 6 & 11 & 1052.3349 & 3 & \\
\hline 1 & 1 & 0 & 2 & 1 & 1 & 1052.3400 & 6 & \\
\hline 15 & 9 & 7 & 15 & 9 & 6 & 1052.3744 & 4 & \\
\hline 1 & 0 & 1 & 2 & 0 & 2 & 1052.3917 & 4 & \\
\hline 14 & 10 & 5 & 14 & 10 & 4 & 1052.4162 & 5 & \\
\hline 1 & 1 & 1 & 2 & 1 & 2 & 1052.4213 & 7 & \\
\hline 13 & 11 & 3 & 13 & 11 & 2 & 1052.4361 & 2 & \\
\hline 16 & 7 & 10 & 16 & 7 & 9 & 1052.4661 & -15 & 10 \\
\hline 17 & 5 & 13 & 17 & 5 & 12 & 1052.4661 & 19 & 1 \\
\hline 12 & 12 & 1 & 12 & 12 & 0 & 1052.4915 & -2 & \\
\hline 15 & 8 & 8 & 15 & 8 & 7 & 1052.5354 & -10 & \\
\hline 17 & 4 & 14 & 17 & 4 & 13 & 1052.5655 & 4 & \\
\hline 17 & 3 & 15 & 17 & 3 & 14 & 1052.5782 & -8 & \\
\hline 16 & 6 & 11 & 16 & 6 & 10 & 1052.6447 & -6 & \\
\hline 14 & 9 & 6 & 14 & 9 & 5 & 1052.6652 & 2 & \\
\hline 13 & 10 & 4 & 13 & 10 & 3 & 1052.7048 & 5 & \\
\hline 12 & 11 & 2 & 12 & 11 & 1 & 1052.7365 & 3 & \\
\hline 15 & 7 & 9 & 15 & 7 & 8 & 1052.7659 & -8 & \\
\hline 16 & 5 & 12 & 16 & 5 & 11 & 1052.7755 & 1 & \\
\hline 16 & 4 & 13 & 16 & 4 & 12 & 1052.8774 & 1 & \\
\hline 16 & 3 & 14 & 16 & 3 & 13 & 1052.9089 & -5 & \\
\hline 14 & 8 & 7 & 14 & 8 & 6 & 1052.9347 & 15 & 1 \\
\hline 15 & 6 & 10 & 15 & 6 & 9 & 1052.9385 & -1 & \\
\hline 13 & 9 & 5 & 13 & 9 & 4 & 1052.9385 & 1 & \\
\hline 12 & 10 & 3 & 12 & 10 & 2 & 1052.9753 & 3 & \\
\hline 11 & 11 & 1 & 11 & 11 & 0 & 1053.0187 & 5 & \\
\hline 14 & 7 & 8 & 14 & 7 & 7 & 1053.0473 & -7 & \\
\hline 15 & 5 & 11 & 15 & 5 & 10 & 1053.0684 & 0 & \\
\hline 14 & 2 & 13 & 14 & 2 & 12 & 1053.1072 & -2 & \\
\hline 13 & 8 & 6 & 13 & 8 & 5 & 1053.1654 & 7 & \\
\hline
\end{tabular}




\begin{tabular}{|c|c|c|c|c|c|c|c|}
\hline 15 & 4 & 12 & 15 & 4 & 11 & 1053.1711 & 1 \\
\hline 12 & 9 & 4 & 12 & 9 & 3 & 1053.1944 & 3 \\
\hline 14 & 6 & 9 & 14 & 6 & 8 & 1053.2141 & -2 \\
\hline 11 & 10 & 2 & 11 & 10 & 1 & 1053.2277 & 4 \\
\hline 15 & 3 & 12 & 15 & 3 & 13 & 1053.2598 & -2 \\
\hline 13 & 7 & 7 & 13 & 7 & 6 & 1053.3107 & -5 \\
\hline 14 & 5 & 10 & 14 & 5 & 9 & 1053.3435 & 1 \\
\hline 12 & 8 & 5 & 12 & 8 & 4 & 1053.3994 & 4 \\
\hline 11 & 9 & 3 & 11 & 9 & 2 & 1053.4318 & 3 \\
\hline 14 & 4 & 11 & 14 & 4 & 10 & 1053.4460 & -1 \\
\hline 10 & 10 & 1 & 10 & 10 & 0 & 1053.4611 & 5 \\
\hline 13 & 6 & 8 & 13 & 6 & 7 & 1053.4719 & -4 \\
\hline 13 & 2 & 12 & 13 & 2 & 11 & 1053.4796 & 0 \\
\hline 14 & 3 & 12 & 14 & 3 & 11 & 1053.5019 & -3 \\
\hline 14 & 3 & 11 & 14 & 3 & 12 & 1053.5308 & -3 \\
\hline 12 & 7 & 6 & 12 & 7 & 5 & 1053.5559 & -4 \\
\hline 13 & 5 & 9 & 13 & 5 & 8 & 1053.6004 & 1 \\
\hline 11 & 8 & 4 & 11 & 8 & 3 & 1053.6232 & 3 \\
\hline 10 & 9 & 2 & 10 & 9 & 1 & 1053.6508 & 3 \\
\hline 13 & 4 & 10 & 13 & 4 & 9 & 1053.7027 & -2 \\
\hline 12 & 6 & 7 & 12 & 6 & 6 & 1053.7120 & -4 \\
\hline 13 & 3 & 11 & 13 & 3 & 10 & 1053.7662 & -2 \\
\hline 11 & 7 & 5 & 11 & 7 & 4 & 1053.7830 & -1 \\
\hline 15 & 2 & 13 & 15 & 2 & 14 & 1053.7985 & -1 \\
\hline 12 & 2 & 11 & 12 & 2 & 10 & 1053.8117 & 0 \\
\hline 10 & 8 & 3 & 10 & 8 & 2 & 1053.8318 & 1 \\
\hline 12 & 5 & 8 & 12 & 5 & 7 & 1053.8382 & -8 \\
\hline 9 & 9 & 1 & 9 & 9 & 0 & 1053.8509 & 3 \\
\hline 11 & 6 & 6 & 11 & 6 & 5 & 1053.9342 & -2 \\
\hline 12 & 4 & 9 & 12 & 4 & 8 & 1053.9411 & -1 \\
\hline 14 & 2 & 12 & 14 & 2 & 13 & 1053.9603 & -1 \\
\hline 0 & 0 & 0 & 1 & 0 & 1 & 1053.9774 & 5 \\
\hline 10 & 7 & 4 & 10 & 7 & 3 & 1053.9911 & -1 \\
\hline 12 & 3 & 10 & 12 & 3 & 9 & 1054.0097 & -3 \\
\hline 9 & 8 & 2 & 9 & 8 & 1 & 1054.0235 & 0 \\
\hline 11 & 5 & 7 & 11 & 5 & 6 & 1054.0591 & -4 \\
\hline 11 & 2 & 10 & 11 & 2 & 9 & 1054.1059 & -2 \\
\hline 13 & 2 & 11 & 13 & 2 & 12 & 1054.1240 & -1 \\
\hline 10 & 6 & 5 & 10 & 6 & 4 & 1054.1381 & -2 \\
\hline 11 & 4 & 8 & 11 & 4 & 7 & 1054.1610 & -2 \\
\hline 9 & 7 & 3 & 9 & 7 & 2 & 1054.1806 & -1 \\
\hline 8 & 8 & 1 & 8 & 8 & 0 & 1054.1974 & 1 \\
\hline 11 & 3 & 9 & 11 & 3 & 8 & 1054.2330 & -5 \\
\hline 11 & 3 & 8 & 11 & 3 & 9 & 1054.2405 & 0 \\
\hline 10 & 5 & 6 & 10 & 5 & 5 & 1054.2615 & -3 \\
\hline 12 & 2 & 10 & 12 & 2 & 11 & 1054.2872 & 1 \\
\hline 9 & 6 & 4 & 9 & 6 & 3 & 1054.3238 & -2 \\
\hline 8 & 7 & 2 & 8 & 7 & 1 & 1054.3514 & -1 \\
\hline 10 & 4 & 7 & 10 & 4 & 6 & 1054.3628 & 0 \\
\hline 10 & 3 & 8 & 10 & 3 & 7 & 1054.4368 & -6 \\
\hline 10 & 3 & 7 & 10 & 3 & 8 & 1054.4412 & -2 \\
\hline 9 & 5 & 5 & 9 & 5 & 4 & 1054.4457 & -1 \\
\hline 8 & 6 & 3 & 8 & 6 & 2 & 1054.4912 & -1 \\
\hline 7 & 7 & 1 & 7 & 7 & 0 & 1054.5033 & 1 \\
\hline 9 & 4 & 6 & 9 & 4 & 5 & 1054.5460 & -1 \\
\hline 9 & 2 & 8 & 9 & 2 & 7 & 1054.5922 & 1 \\
\hline 10 & 2 & 8 & 10 & 2 & 9 & 1054.6028 & 3 \\
\hline 8 & 5 & 4 & 8 & 5 & 3 & 1054.6115 & 0 \\
\hline 9 & 3 & 7 & 9 & 3 & 6 & 1054.6230 & 10 \\
\hline 7 & 6 & 2 & 7 & 6 & 1 & 1054.6402 & 0 \\
\hline 8 & 4 & 5 & 8 & 4 & 4 & 1054.7110 & 0 \\
\hline 9 & 2 & 7 & 9 & 2 & 8 & 1054.7510 & 4 \\
\hline 7 & 5 & 3 & 7 & 5 & 2 & 1054.7589 & 0 \\
\hline 6 & 6 & 1 & 6 & 6 & 0 & 1054.7706 & 0 \\
\hline 8 & 3 & 6 & 8 & 3 & 5 & 1054.7882 & 6 \\
\hline 7 & 4 & 4 & 7 & 4 & 3 & 1054.8577 & 1 \\
\hline 6 & 5 & 2 & 6 & 5 & 1 & 1054.8882 & 2 \\
\hline 7 & 3 & 5 & 7 & 3 & 4 & 1054.9347 & 3 \\
\hline 7 & 2 & 6 & 7 & 2 & 5 & 1054.9573 & 3 \\
\hline 6 & 4 & 3 & 6 & 4 & 2 & 1054.9860 & 1 \\
\hline 5 & 5 & 1 & 5 & 5 & 0 & 1054.9988 & 2 \\
\hline 7 & 2 & 5 & 7 & 2 & 6 & 1055.0179 & 1 \\
\hline 4 & 1 & 4 & 4 & 1 & 3 & 1055.0363 & 3 \\
\hline 6 & 3 & 4 & 6 & 3 & 3 & 1055.0629 & 3 \\
\hline 5 & 4 & 2 & 5 & 4 & 1 & 1055.0961 & 3 \\
\hline 6 & 2 & 4 & 6 & 2 & 5 & 1055.1336 & 1 \\
\hline 5 & 3 & 3 & 5 & 3 & 2 & 1055.1727 & 3 \\
\hline
\end{tabular}




\begin{tabular}{|c|c|c|c|c|c|c|c|}
\hline 10 & 6 & 4 & 9 & 6 & 3 & 1069.8037 & -2 \\
\hline 10 & 5 & 5 & 9 & 5 & 4 & 1069.9303 & -2 \\
\hline 10 & 4 & 6 & 9 & 4 & 5 & 1070.0348 & -1 \\
\hline 10 & 3 & 7 & 9 & 3 & 6 & 1070.1160 & 0 \\
\hline 10 & 1 & 9 & 9 & 1 & 8 & 1070.1545 & 3 \\
\hline 10 & 2 & 8 & 9 & 2 & 7 & 1070.1658 & 0 \\
\hline 10 & 2 & 9 & 9 & 2 & 8 & 1070.1780 & -1 \\
\hline 10 & 0 & 10 & 9 & 0 & 9 & 1070.2385 & 0 \\
\hline 10 & 1 & 10 & 9 & 1 & 9 & 1070.2772 & -2 \\
\hline 11 & 10 & 1 & 10 & 10 & 0 & 1070.4414 & 5 \\
\hline 11 & 9 & 2 & 10 & 9 & 1 & 1070.6504 & 3 \\
\hline 11 & 8 & 3 & 10 & 8 & 2 & 1070.8463 & 3 \\
\hline 11 & 7 & 4 & 10 & 7 & 3 & 1071.0099 & -3 \\
\hline 11 & 6 & 5 & 10 & 6 & 4 & 1071.1651 & -3 \\
\hline 11 & 5 & 6 & 10 & 5 & 5 & 1071.2938 & -1 \\
\hline 11 & 4 & 7 & 10 & 4 & 6 & 1071.3997 & -1 \\
\hline 11 & 3 & 8 & 10 & 3 & 7 & 1071.4812 & 1 \\
\hline 11 & 1 & 10 & 10 & 1 & 9 & 1071.4955 & 2 \\
\hline 12 & 11 & 1 & 11 & 11 & 0 & 1071.5075 & 4 \\
\hline 11 & 2 & 9 & 10 & 2 & 8 & 1071.5240 & -1 \\
\hline 11 & 2 & 10 & 10 & 2 & 9 & 1071.5469 & -1 \\
\hline 11 & 0 & 11 & 10 & 0 & 10 & 1071.6179 & -1 \\
\hline 11 & 1 & 11 & 10 & 1 & 10 & 1071.6775 & -4 \\
\hline 12 & 10 & 2 & 11 & 10 & 1 & 1071.7525 & 6 \\
\hline 12 & 9 & 3 & 11 & 9 & 2 & 1071.9766 & 3 \\
\hline 12 & 8 & 4 & 11 & 8 & 3 & 1072.1866 & 4 \\
\hline 12 & 7 & 5 & 11 & 7 & 4 & 1072.3477 & -4 \\
\hline 13 & 12 & 1 & 12 & 12 & 0 & 1072.4450 & -8 \\
\hline 12 & 6 & 6 & 11 & 6 & 5 & 1072.5081 & -3 \\
\hline 12 & 5 & 7 & 11 & 5 & 6 & 1072.6381 & 9 \\
\hline 12 & 4 & 8 & 11 & 4 & 7 & 1072.7459 & -3 \\
\hline 12 & 1 & 11 & 11 & 1 & 10 & 1072.8157 & 2 \\
\hline 12 & 3 & 9 & 11 & 3 & 8 & 1072.8275 & 3 \\
\hline 12 & 2 & 10 & 11 & 2 & 9 & 1072.8603 & -1 \\
\hline 12 & 2 & 11 & 11 & 2 & 10 & 1072.8983 & -2 \\
\hline 12 & 0 & 12 & 11 & 0 & 11 & 1072.9839 & -2 \\
\hline 13 & 10 & 3 & 12 & 10 & 2 & 1073.0444 & 5 \\
\hline 12 & 1 & 12 & 11 & 1 & 11 & 1073.0650 & -4 \\
\hline 13 & 9 & 4 & 12 & 9 & 3 & 1073.2842 & 2 \\
\hline 13 & 8 & 5 & 12 & 8 & 4 & 1073.5163 & 6 \\
\hline 14 & 12 & 2 & 13 & 12 & 1 & 1073.6208 & -16 \\
\hline 13 & 7 & 6 & 12 & 7 & 5 & 1073.6666 & -6 \\
\hline 13 & 6 & 7 & 12 & 6 & 6 & 1073.8326 & -4 \\
\hline 13 & 5 & 8 & 12 & 5 & 7 & 1073.9658 & -8 \\
\hline 14 & 11 & 3 & 13 & 11 & 2 & 1074.0132 & 1 \\
\hline 13 & 4 & 9 & 12 & 4 & 8 & 1074.0736 & -3 \\
\hline 13 & 1 & 12 & 12 & 1 & 11 & 1074.1154 & 1 \\
\hline 13 & 3 & 10 & 12 & 3 & 9 & 1074.1550 & 8 \\
\hline 13 & 2 & 11 & 12 & 2 & 10 & 1074.1740 & -1 \\
\hline 13 & 2 & 12 & 12 & 2 & 11 & 1074.2327 & -2 \\
\hline 14 & 10 & 4 & 13 & 10 & 3 & 1074.3181 & 4 \\
\hline 13 & 0 & 13 & 12 & 0 & 12 & 1074.3376 & -2 \\
\hline 13 & 1 & 13 & 12 & 1 & 12 & 1074.4399 & -5 \\
\hline 14 & 9 & 5 & 13 & 9 & 4 & 1074.5736 & 3 \\
\hline 15 & 12 & 3 & 14 & 12 & 2 & 1074.7850 & -19 \\
\hline 14 & 8 & 6 & 13 & 8 & 5 & 1074.8486 & 11 \\
\hline 14 & 7 & 7 & 13 & 7 & 6 & 1074.9670 & -7 \\
\hline 14 & 6 & 8 & 13 & 6 & 7 & 1075.1389 & -4 \\
\hline 15 & 11 & 4 & 14 & 11 & 3 & 1075.2401 & 1 \\
\hline 14 & 5 & 9 & 13 & 5 & 8 & 1075.2737 & -9 \\
\hline 14 & 4 & 10 & 13 & 4 & 9 & 1075.3826 & -4 \\
\hline 14 & 1 & 13 & 13 & 1 & 12 & 1075.3952 & 0 \\
\hline 14 & 3 & 11 & 13 & 3 & 10 & 1075.4640 & 20 \\
\hline 14 & 2 & 13 & 13 & 2 & 12 & 1075.5500 & -3 \\
\hline 15 & 10 & 5 & 14 & 10 & 4 & 1075.5740 & 5 \\
\hline 14 & 0 & 14 & 13 & 0 & 13 & 1075.6798 & -3 \\
\hline 14 & 1 & 14 & 13 & 1 & 13 & 1075.8024 & -5 \\
\hline 15 & 9 & 6 & 14 & 9 & 5 & 1075.8450 & 4 \\
\hline 16 & 12 & 4 & 15 & 12 & 3 & 1075.9382 & -20 \\
\hline 15 & 8 & 7 & 14 & 8 & 6 & 1076.0125 & -9 \\
\hline 15 & 7 & 8 & 14 & 7 & 7 & 1076.2489 & -8 \\
\hline 15 & 6 & 9 & 14 & 6 & 8 & 1076.4269 & -5 \\
\hline 16 & 11 & 5 & 15 & 11 & 4 & 1076.4506 & 1 \\
\hline 15 & 5 & 10 & 14 & 5 & 9 & 1076.5633 & -10 \\
\hline 15 & 1 & 14 & 14 & 1 & 13 & 1076.6558 & 0 \\
\hline 15 & 4 & 11 & 14 & 4 & 10 & 1076.6731 & -4 \\
\hline 15 & 2 & 13 & 14 & 2 & 12 & 1076.7310 & -1 \\
\hline
\end{tabular}

\begin{tabular}{|c|c|}
\hline 1076.749 & -4 \\
\hline 1076.7560 & -2 \\
\hline 1076.8123 & 5 \\
\hline 1076.8507 & -3 \\
\hline .077 .0114 & -4 \\
\hline 1077.0808 & -22 \\
\hline 1077.0987 & 4 \\
\hline 1077.1527 & -6 \\
\hline 1077.2892 & -7 \\
\hline 1077.5125 & -10 \\
\hline 1077.6457 & 4 \\
\hline 1077.6970 & -5 \\
\hline 1077.8345 & -12 \\
\hline 1077.8977 & 0 \\
\hline 1077.9450 & -3 \\
\hline 1077.9734 & -1 \\
\hline 1078.0181 & -4 \\
\hline 1078.0276 & -4 \\
\hline 1078.0342 & 9 \\
\hline 1078.1350 & -3 \\
\hline 1078.2137 & -22 \\
\hline 1078.3339 & -7 \\
\hline 1078.3339 & 2 \\
\hline 1078.4912 & -5 \\
\hline 1078.5407 & -5 \\
\hline 1078.7581 & -10 \\
\hline 1078.8255 & 4 \\
\hline 1078.9492 & -6 \\
\hline 1079.0873 & -15 \\
\hline 1079.1216 & 0 \\
\hline 1079.1913 & -2 \\
\hline 1079.1982 & -2 \\
\hline 1079.2389 & 7 \\
\hline 1079.2663 & -4 \\
\hline 1079.2807 & -4 \\
\hline 1079.3371 & -23 \\
\hline 1079.4032 & -3 \\
\hline 1079.5545 & 6 \\
\hline 1079.6463 & -3 \\
\hline 1079.7722 & -3 \\
\hline 1079.8179 & -4 \\
\hline 1079.9857 & -11 \\
\hline 1079.9917 & 12 \\
\hline 1080.1837 & -8 \\
\hline 1080.3216 & -21 \\
\hline 1080.3283 & 0 \\
\hline 1080.3847 & -1 \\
\hline 1080.4276 & 5 \\
\hline 1080.4324 & -2 \\
\hline 1080.4521 & -19 \\
\hline 1080.4940 & -3 \\
\hline 1080.5152 & -3 \\
\hline 1080.6556 & -3 \\
\hline 1080.7571 & 5 \\
\hline 1080.9507 & -2 \\
\hline 1080.9859 & 0 \\
\hline 1081.1331 & -3 \\
\hline 1081.1430 & 8 \\
\hline 1081.1957 & -11 \\
\hline 1081.4010 & -8 \\
\hline 1081.5185 & -1 \\
\hline 1081.5376 & -27 \\
\hline 1081.5535 & -1 \\
\hline 1081.5589 & -12 \\
\hline 1081.6014 & 9 \\
\hline 1081.6480 & -1 \\
\hline 1081.7008 & -3 \\
\hline 1081.7310 & -3 \\
\hline 1081.8926 & -2 \\
\hline 1081.9437 & 7 \\
\hline 1082.1828 & 3 \\
\hline 1082.2470 & -1 \\
\hline 1082.2816 & 9 \\
\hline 1082.3882 & -11 \\
\hline 1082.4371 & -1 \\
\hline 1082.6014 & -9 \\
\hline 1082.6570 & -12 \\
\hline
\end{tabular}

$1082.6570-12 \quad 4$ $\begin{array}{llllll}15 & 3 & 12 & 14 & 3 & 11\end{array}$ $\begin{array}{rrrrrr}16 & 3 & 13 & 14 & 3 & 12 \\ 10 & 6 & 15 & 10 & 5\end{array}$ $\begin{array}{llllll}15 & 2 & 14 & 14 & 2 & 13 \\ 15 & 0 & 15 & 14 & 0 & 14\end{array}$ $\begin{array}{rrrrrr}17 & 12 & 5 & 16 & 12 & 4 \\ 16 & 9 & 7 & 15 & 9 & 6\end{array}$ $\begin{array}{rrrrrr}15 & 1 & 15 & 14 & 1 & 14 \\ 16 & 8 & 8 & 15 & 8 & 7\end{array}$ $\begin{array}{lllll}16 & 7 & 9 & 15 & 7\end{array}$ $\begin{array}{rrrrr}17 & 11 & 6 & 16 & 11\end{array}$ $\begin{array}{lllll}16 & 6 & 10 & 15 & 6 \\ 16 & 5 & 11 & 15 & 5\end{array}$ $\begin{array}{lllll}16 & 1 & 15 & 15 & 1 \\ 16 & 4 & 12 & 15 & 4\end{array}$ $\begin{array}{llllll}16 & 2 & 14 & 15 & 2 & 13\end{array}$ $\begin{array}{llllll}16 & 3 & 13 & 15 & 3 & 12\end{array}$ $\begin{array}{rrrrr}17 & 10 & 7 & 16 & 10\end{array}$ $\begin{array}{rrrrr}16 & 2 & 15 & 15 & 2 \\ 18 & 12 & 6 & 17 & 12\end{array}$ $\begin{array}{rrrrr}17 & 9 & 8 & 16 & 9 \\ 16 & 0 & 16 & 15 & 0\end{array}$ $\begin{array}{rrrrr}16 & 1 & 16 & 15 & 1\end{array}$ $\begin{array}{rrrrr}17 & 7 & 10 & 16 & 7 \\ 18 & 11 & 7 & 17 & 11\end{array}$ $\begin{array}{lllll}17 & 6 & 11 & 16 & 6\end{array}$ $\begin{array}{lllll}17 & 5 & 12 & 16 & 5 \\ 17 & 1 & 16 & 16 & 1\end{array}$ $\begin{array}{llllll}17 & 2 & 15 & 16 & 2\end{array}$ $\begin{array}{rrrrr}17 & 4 & 13 & 16 & 4 \\ 18 & 10 & 8 & 17 & 10\end{array}$ $\begin{array}{rrrrrr}17 & 3 & 14 & 16 & 3 & 13\end{array}$ $\begin{array}{rrrrr}17 & 3 & 15 & 16 & \\ 19 & 12 & 7 & 18 & 12\end{array}$ $\begin{array}{rrrrr}17 & 2 & 16 & 16 & 2 \\ 18 & 9 & 9 & 17 & 9\end{array}$ 17 18 18 18 18

18

(a)

(a)

(10)

18

(a)

(a)

(2)

(a)

10

(1)

2

1

19

20

2

$\begin{array}{lll}0 & 17 & 16 \\ 8 & 10 & 17\end{array}$

$\begin{array}{lll}1 & 17 & 16\end{array}$

$\begin{array}{rrrr}11 & 11 & 17 & 7 \\ 6 & 8 & 18 & 11\end{array}$

$\begin{array}{llll}5 & 13 & 17 & 5\end{array}$

$\begin{array}{llll}1 & 17 & 17 & 1 \\ 2 & 16 & 17\end{array}$

$\begin{array}{rrrr}10 & 9 & 18 & 10\end{array}$

$\begin{array}{rrrr}12 & 8 & 19 & 12 \\ 3 & 15 & 17 & 3\end{array}$

$\begin{array}{llll}3 & 16 & 17 & 3\end{array}$

$\begin{array}{llll}2 & 17 & 17 & 2 \\ 9 & 10 & 18 & 9\end{array}$

$\begin{array}{lllll}0 & 18 & 17 & 0 & 17\end{array}$

$\begin{array}{lllll}8 & 11 & 18 & 8 & 10\end{array}$

$\begin{array}{rrrr}11 & 9 & 19 & 11\end{array}$

$\begin{array}{lllll}7 & 12 & 18 & 7 & 11\end{array}$

$\begin{array}{lllll}1 & 18 & 18 & 1 & 17\end{array}$

$\begin{array}{lllll}5 & 14 & 18 & 5 & 13\end{array}$

$\begin{array}{rrrr}12 & 9 & 20 & 12\end{array}$

$\begin{array}{llll}10 & 10 & 19 & 10\end{array}$

$\begin{array}{llll}3 & 15 & 18 & 18 \\ 3 & 17 & 18 & 3\end{array}$

$\begin{array}{lll}3 & 17 & 18\end{array}$

$\begin{array}{lll}2 & 18 & 18 \\ 9 & 11 & 19\end{array}$

$812 \quad 19$

$\begin{array}{llll}0 & 19 & 18 & 0\end{array}$

2

$\begin{array}{rrrr}7 & 13 & 19 & 11\end{array}$

$\begin{array}{lllll}1 & 19 & 18 & 1 & 18 \\ 6 & 14 & 19 & 6 & 13\end{array}$

$\begin{array}{rrrr}12 & 10 & 21 & 12\end{array}$ 


\begin{tabular}{|c|c|c|c|c|c|c|c|}
\hline 20 & 1 & 19 & 19 & 1 & 18 & 1082.6932 & -2 \\
\hline 20 & 2 & 18 & 19 & 2 & 17 & 1082.6983 & 0 \\
\hline 20 & 5 & 16 & 19 & 5 & 15 & 1082.7331 & -7 \\
\hline 20 & 5 & 15 & 19 & 5 & 14 & 1082.7383 & -5 \\
\hline 21 & 10 & 11 & 20 & 10 & 10 & 1082.7598 & 11 \\
\hline 20 & 4 & 16 & 19 & 4 & 15 & 1082.8448 & 2 \\
\hline 20 & 3 & 17 & 19 & 3 & 16 & 1082.8861 & -3 \\
\hline 20 & 3 & 18 & 19 & 3 & 17 & 1082.9285 & -2 \\
\hline 20 & 2 & 19 & 19 & 2 & 18 & 1083.1144 & -2 \\
\hline 21 & 9 & 12 & 20 & 9 & 11 & 1083.1144 & 9 \\
\hline 21 & 8 & 13 & 20 & 8 & 12 & 1083.3637 & 5 \\
\hline 22 & 11 & 11 & 21 & 11 & 10 & 1083.4077 & 13 \\
\hline 20 & 0 & 20 & 19 & 0 & 19 & 1083.5353 & 1 \\
\hline 21 & 7 & 14 & 20 & 7 & 13 & 1083.5634 & -11 \\
\hline 20 & 1 & 20 & 19 & 1 & 19 & 1083.7300 & 1 \\
\hline 23 & 12 & 11 & 22 & 12 & 10 & 1083.7478 & -8 \\
\hline 21 & 6 & 15 & 20 & 6 & 14 & 1083.7853 & -12 \\
\hline 21 & 2 & 19 & 20 & 2 & 18 & 1083.8191 & 0 \\
\hline 21 & 1 & 20 & 20 & 1 & 19 & 1083.8536 & 0 \\
\hline 22 & 10 & 12 & 21 & 10 & 11 & 1083.9036 & 14 \\
\hline 21 & 5 & 17 & 20 & 5 & 16 & 1083.9119 & -6 \\
\hline 21 & 5 & 16 & 20 & 5 & 15 & 1083.9186 & -4 \\
\hline 21 & 4 & 17 & 20 & 4 & 16 & 1084.0227 & 6 \\
\hline 21 & 3 & 18 & 20 & 3 & 17 & 1084.0497 & -1 \\
\hline 21 & 3 & 19 & 20 & 3 & 18 & 1084.1076 & -2 \\
\hline 22 & 9 & 13 & 21 & 9 & 12 & 1084.2691 & 8 \\
\hline 21 & 2 & 20 & 20 & 2 & 19 & 1084.3216 & 0 \\
\hline 23 & 11 & 12 & 22 & 11 & 11 & 1084.5215 & 15 \\
\hline 22 & 8 & 14 & 21 & 8 & 13 & 1084.5295 & 7 \\
\hline 22 & 7 & 15 & 21 & 7 & 14 & 1084.7215 & -12 \\
\hline 21 & 0 & 21 & 20 & 0 & 20 & 1084.8158 & 4 \\
\hline 24 & 12 & 12 & 23 & 12 & 11 & 1084.8315 & -1 \\
\hline 22 & 2 & 20 & 21 & 2 & 19 & 1084.9168 & 0 \\
\hline 22 & 6 & 16 & 21 & 6 & 15 & 1084.9536 & -17 \\
\hline 22 & 1 & 21 & 21 & 1 & 20 & 1085.0004 & 0 \\
\hline 21 & 1 & 21 & 20 & 1 & 20 & 1085.0120 & 4 \\
\hline 23 & 10 & 13 & 22 & 10 & 12 & 1085.0328 & 14 \\
\hline 22 & 5 & 18 & 21 & 5 & 17 & 1085.0723 & -5 \\
\hline 22 & 5 & 17 & 21 & 5 & 16 & 1085.0807 & -4 \\
\hline 22 & 3 & 19 & 21 & 3 & 18 & 1085.1907 & -1 \\
\hline 22 & 3 & 20 & 21 & 3 & 19 & 1085.2686 & -1 \\
\hline 23 & 9 & 14 & 22 & 9 & 13 & 1085.4087 & 8 \\
\hline 22 & 2 & 21 & 21 & 2 & 20 & 1085.5142 & 1 \\
\hline 24 & 11 & 13 & 23 & 11 & 12 & 1085.6234 & 16 \\
\hline 23 & 8 & 15 & 22 & 8 & 14 & 1085.6809 & 10 \\
\hline 23 & 7 & 16 & 22 & 7 & 15 & 1085.8629 & -11 \\
\hline 25 & 12 & 13 & 24 & 12 & 12 & 1085.9075 & 0 \\
\hline 23 & 2 & 21 & 22 & 2 & 20 & 1085.9922 & 1 \\
\hline 22 & 0 & 22 & 21 & 0 & 21 & 1086.0883 & 7 \\
\hline 23 & 6 & 18 & 22 & 6 & 17 & 1086.1054 & -5 \\
\hline 23 & 6 & 17 & 22 & 6 & 16 & 1086.1095 & -8 \\
\hline 23 & 1 & 22 & 22 & 1 & 21 & 1086.1347 & 1 \\
\hline 24 & 10 & 14 & 23 & 10 & 13 & 1086.1482 & 14 \\
\hline 23 & 5 & 19 & 22 & 5 & 18 & 1086.2142 & -4 \\
\hline 23 & 5 & 18 & 22 & 5 & 17 & 1086.2247 & -4 \\
\hline 22 & 1 & 22 & 21 & 1 & 21 & 1086.2833 & 8 \\
\hline 23 & 3 & 20 & 22 & 3 & 19 & 1086.3088 & 0 \\
\hline 23 & 4 & 19 & 22 & 4 & 18 & 1086.3196 & -1 \\
\hline 23 & 4 & 20 & 22 & 4 & 19 & 1086.3238 & -3 \\
\hline 23 & 3 & 21 & 22 & 3 & 20 & 1086.4116 & 0 \\
\hline 24 & 9 & 15 & 23 & 9 & 14 & 1086.5334 & 8 \\
\hline 23 & 2 & 22 & 22 & 2 & 21 & 1086.6929 & 4 \\
\hline 25 & 11 & 14 & 24 & 11 & 13 & 1086.7142 & 18 \\
\hline 24 & 8 & 16 & 23 & 8 & 15 & 1086.8188 & 12 \\
\hline 26 & 12 & 14 & 25 & 12 & 13 & 1086.9775 & 9 \\
\hline 24 & 7 & 17 & 23 & 7 & 16 & 1086.9878 & -8 \\
\hline 24 & 2 & 22 & 23 & 2 & 21 & 1087.0460 & 1 \\
\hline 24 & 6 & 19 & 23 & 6 & 18 & 1087.2440 & -6 \\
\hline 25 & 10 & 15 & 24 & 10 & 14 & 1087.2501 & 15 \\
\hline 24 & 6 & 18 & 23 & 6 & 17 & 1087.2527 & -15 \\
\hline 24 & 1 & 23 & 23 & 1 & 22 & 1087.2576 & 1 \\
\hline 24 & 5 & 20 & 23 & 5 & 19 & 1087.3376 & -3 \\
\hline 24 & 3 & 21 & 23 & 3 & 20 & 1087.4033 & 1 \\
\hline 24 & 4 & 20 & 23 & 4 & 19 & 1087.4395 & -1 \\
\hline 24 & 4 & 21 & 23 & 4 & 20 & 1087.4459 & 1 \\
\hline 24 & 3 & 22 & 23 & 3 & 21 & 1087.5370 & 2 \\
\hline 25 & 9 & 16 & 24 & 9 & 15 & 1087.6435 & 6 \\
\hline
\end{tabular}

\begin{tabular}{|c|c|c|c|c|c|c|c|c|}
\hline 26 & 11 & 15 & 25 & 11 & 14 & 1087.7939 & 19 & 4 \\
\hline 24 & 2 & 23 & 23 & 2 & 22 & 1087.8578 & 6 & 4 \\
\hline 25 & 8 & 17 & 24 & 8 & 16 & 1087.9443 & 14 & 1 \\
\hline 27 & 12 & 15 & 26 & 12 & 14 & 1088.0402 & 10 & 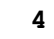 \\
\hline 25 & 2 & 23 & 24 & 2 & 22 & 1088.0792 & 2 & 4 \\
\hline 25 & 7 & 18 & 24 & 7 & 17 & 1088.0962 & -8 & 4 \\
\hline 26 & 10 & 16 & 25 & 10 & 15 & 1088.3386 & 12 & 4 \\
\hline 25 & 6 & 20 & 24 & 6 & 19 & 1088.3705 & -7 & 4 \\
\hline 25 & 1 & 24 & 24 & 1 & 23 & 1088.3705 & 5 & 4 \\
\hline 25 & 6 & 19 & 24 & 6 & 18 & 1088.3922 & -8 & 4 \\
\hline 25 & 5 & 21 & 24 & 5 & 20 & 1088.4426 & -2 & . \\
\hline 25 & 5 & 20 & 24 & 5 & 19 & 1088.4584 & -2 & 4 \\
\hline 25 & 3 & 22 & 24 & 3 & 21 & 1088.4736 & 2 & . \\
\hline 25 & 4 & 21 & 24 & 4 & 20 & 1088.5400 & 1 & . \\
\hline 25 & 4 & 22 & 24 & 4 & 21 & 1088.5489 & 1 & 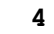 \\
\hline 25 & 3 & 23 & 24 & 3 & 22 & 1088.6449 & 4 & 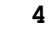 \\
\hline 26 & 9 & 17 & 25 & 9 & 16 & 1088.7393 & 4 & 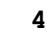 \\
\hline 27 & 11 & 16 & 26 & 11 & 15 & 1088.8630 & 18 & 4 \\
\hline 25 & 2 & 24 & 24 & 2 & 23 & 1089.0095 & 10 & 4 \\
\hline 26 & 8 & 18 & 25 & 8 & 17 & 1089.0584 & 12 & 4 \\
\hline 26 & 7 & 19 & 25 & 7 & 18 & 1089.1885 & -7 & 4 \\
\hline 27 & 10 & 17 & 26 & 10 & 16 & 1089.4144 & 9 & 4 \\
\hline 26 & 6 & 21 & 25 & 6 & 20 & 1089.4884 & -2 & 4 \\
\hline 26 & 3 & 23 & 25 & 3 & 22 & 1089.5192 & 3 & 4 \\
\hline 26 & 5 & 22 & 25 & 5 & 21 & 1089.5290 & -2 & 4 \\
\hline 26 & 6 & 20 & 25 & 6 & 19 & 1089.5398 & -10 & 4 \\
\hline 26 & 5 & 21 & 25 & 5 & 20 & 1089.5481 & -1 & 4 \\
\hline 26 & 4 & 22 & 25 & 4 & 21 & 1089.6208 & 2 & 4 \\
\hline 26 & 4 & 23 & 25 & 4 & 22 & 1089.6333 & 2 & 4 \\
\hline 26 & 3 & 24 & 25 & 3 & 23 & 1089.7357 & 7 & 4 \\
\hline 27 & 9 & 18 & 26 & 9 & 17 & 1089.8212 & 0 & 4 \\
\hline 28 & 11 & 17 & 27 & 11 & 16 & 1089.9284 & 81 & 4 \\
\hline 27 & 8 & 19 & 26 & 8 & 18 & 1090.1633 & 11 & 4 \\
\hline 27 & 7 & 20 & 26 & 7 & 19 & 1090.2650 & -5 & 4 \\
\hline 28 & 10 & 18 & 27 & 10 & 17 & 1090.4780 & 9 & 4 \\
\hline 27 & 3 & 24 & 26 & 3 & 23 & 1090.5396 & 5 & 4 \\
\hline 27 & 5 & 23 & 26 & 5 & 22 & 1090.5972 & 0 & 4 \\
\hline 27 & 6 & 22 & 26 & 6 & 21 & 1090.6016 & 5 & 4 \\
\hline 27 & 5 & 22 & 26 & 5 & 21 & 1090.6195 & -1 & 4 \\
\hline 27 & 4 & 23 & 26 & 4 & 22 & 1090.6818 & 4 & 4 \\
\hline 27 & 4 & 24 & 26 & 4 & 23 & 1090.6989 & 4 & 4 \\
\hline 27 & 6 & 21 & 26 & 6 & 20 & 1090.7362 & -18 & 4 \\
\hline 27 & 3 & 25 & 26 & 3 & 24 & 1090.8095 & 9 & 4 \\
\hline 28 & 9 & 19 & 27 & 9 & 18 & 1090.8895 & -5 & 4 \\
\hline 29 & 11 & 18 & 28 & 11 & 17 & 1090.9714 & 18 & 10 \\
\hline 28 & 7 & 21 & 27 & 7 & 20 & 1091.3260 & -3 & 4 \\
\hline 29 & 10 & 19 & 28 & 10 & 18 & 1091.5284 & -4 & 4 \\
\hline 28 & 3 & 25 & 27 & 3 & 24 & 1091.5343 & 7 & 4 \\
\hline 28 & 5 & 24 & 27 & 5 & 23 & 1091.6466 & 0 & 4 \\
\hline 28 & 5 & 23 & 27 & 5 & 22 & 1091.6729 & 1 & 4 \\
\hline 28 & 6 & 23 & 27 & 6 & 22 & 1091.7172 & 9 & 4 \\
\hline 28 & 4 & 24 & 27 & 4 & 23 & 1091.7229 & 7 & 4 \\
\hline 28 & 4 & 25 & 27 & 4 & 24 & 1091.7458 & 5 & 4 \\
\hline 28 & 3 & 26 & 27 & 3 & 25 & 1091.8668 & 11 & 4 \\
\hline 29 & 9 & 20 & 28 & 9 & 19 & 1091.9447 & -11 & 4 \\
\hline 30 & 11 & 19 & 29 & 11 & 18 & 1092.0099 & 5 & 4 \\
\hline 28 & 6 & 22 & 27 & 6 & 21 & 1092.0836 & -47 & 4 \\
\hline 29 & 6 & 23 & 28 & 6 & 22 & 1092.2222 & -39 & 4 \\
\hline 29 & 8 & 21 & 28 & 8 & 20 & 1092.3546 & -3 & 4 \\
\hline 29 & 7 & 22 & 28 & 7 & 21 & 1092.3716 & -2 & 4 \\
\hline 29 & 3 & 26 & 28 & 3 & 25 & 1092.5028 & 7 & 4 \\
\hline 30 & 10 & 20 & 29 & 10 & 19 & 1092.5685 & -4 & 4 \\
\hline 29 & 5 & 25 & 28 & 5 & 24 & 1092.6776 & 0 & 4 \\
\hline 29 & 5 & 24 & 28 & 5 & 23 & 1092.7080 & 1 & 4 \\
\hline 29 & 4 & 26 & 28 & 4 & 25 & 1092.7744 & 10 & 4 \\
\hline 29 & 6 & 24 & 28 & 6 & 23 & 1092.8487 & 27 & 4 \\
\hline 29 & 3 & 27 & 28 & 3 & 26 & 1092.9080 & 14 & 4 \\
\hline 30 & 9 & 21 & 29 & 9 & 20 & 1092.9870 & -20 & 4 \\
\hline 30 & 6 & 24 & 29 & 6 & 23 & 1093.3482 & -28 & 4 \\
\hline 30 & 7 & 23 & 29 & 7 & 22 & 1093.4023 & -1 & 4 \\
\hline 30 & 3 & 27 & 29 & 3 & 26 & 1093.4459 & 15 & 4 \\
\hline 31 & 10 & 21 & 30 & 10 & 20 & 1093.5962 & -13 & 4 \\
\hline 30 & 5 & 25 & 29 & 5 & 24 & 1093.7250 & 2 & 4 \\
\hline 30 & 4 & 26 & 29 & 4 & 25 & 1093.7431 & 10 & 4 \\
\hline 30 & 4 & 27 & 29 & 4 & 26 & 1093.7841 & 11 & 4 \\
\hline 30 & 3 & 28 & 29 & 3 & 27 & 1093.9335 & 18 & 4 \\
\hline 30 & 6 & 25 & 29 & 6 & 24 & 1094.0142 & 64 & \\
\hline
\end{tabular}




$\begin{array}{rrrrrrrrr}31 & 6 & 25 & 30 & 6 & 24 & 1094.4043 & -21 & 4 \\ 31 & 7 & 24 & 30 & 7 & 23 & 1094.4183 & 0 & 4 \\ 32 & 10 & 22 & 31 & 10 & 21 & 1094.6124 & -28 & 4 \\ 31 & 4 & 27 & 30 & 4 & 26 & 1094.7227 & 20 & 10 \\ 31 & 4 & 28 & 30 & 4 & 27 & 1094.7755 & 14 & 4 \\ 32 & 9 & 23 & 31 & 9 & 22 & 1095.0348 & -41 & 4 \\ 31 & 6 & 26 & 30 & 6 & 25 & 1095.2297 & 101 & 4 \\ 32 & 6 & 26 & 31 & 6 & 25 & 1095.4205 & -28 & 4 \\ 32 & 7 & 25 & 31 & 7 & 24 & 1095.4205 & 7 & 4 \\ 33 & 10 & 23 & 32 & 10 & 22 & 1095.6181 & -41 & 4 \\ 32 & 4 & 28 & 31 & 4 & 27 & 1095.6791 & 12 & 4 \\ 32 & 4 & 29 & 31 & 4 & 28 & 1095.7484 & 15 & 4 \\ 33 & 9 & 24 & 32 & 9 & 23 & 1096.0404 & -60 & 4\end{array}$

\begin{tabular}{|c|c|c|c|c|c|c|c|c|}
\hline 26 & 1 & 26 & 27 & 1 & 27 & 892.6606 & 36 & 4 \\
\hline 26 & 0 & 26 & 27 & 0 & 27 & 892.6712 & 24 & 4 \\
\hline 26 & 2 & 25 & 27 & 2 & 26 & 892.7758 & -17 & 4 \\
\hline 25 & 1 & 25 & 26 & 1 & 26 & 894.4962 & 22 & 4 \\
\hline 25 & 0 & 25 & 26 & 0 & 26 & 894.5063 & 19 & 4 \\
\hline 25 & 2 & 24 & 26 & 2 & 25 & 894.5867 & -10 & 4 \\
\hline 25 & 1 & 24 & 26 & 1 & 25 & 894.6417 & -19 & 10 \\
\hline 25 & 2 & 23 & 26 & 2 & 24 & 894.6927 & -20 & 4 \\
\hline 24 & 1 & 24 & 25 & 1 & 25 & 896.3202 & 11 & 4 \\
\hline 24 & 0 & 24 & 25 & 0 & 25 & 896.3283 & 8 & 4 \\
\hline 24 & 2 & 23 & 25 & 2 & 24 & 896.3863 & -14 & 4 \\
\hline 24 & 4 & 20 & 25 & 4 & 21 & 896.4316 & -9 & 4 \\
\hline 24 & 5 & 19 & 25 & 5 & 20 & 896.4406 & 1 & 4 \\
\hline 24 & 6 & 18 & 25 & 6 & 19 & 896.4515 & 5 & 4 \\
\hline 24 & 7 & 17 & 25 & 7 & 18 & 896.4613 & 2 & 10 \\
\hline 24 & 2 & 22 & 25 & 2 & 23 & 896.4613 & 5 & 10 \\
\hline 24 & 8 & 16 & 25 & 8 & 17 & 896.4685 & 1 & 10 \\
\hline 24 & 9 & 15 & 25 & 9 & 16 & 896.4685 & 9 & 10 \\
\hline 23 & 1 & 23 & 24 & 1 & 24 & 898.1326 & 2 & 4 \\
\hline 23 & 0 & 23 & 24 & 0 & 24 & 898.1383 & 2 & 4 \\
\hline 23 & 2 & 22 & 24 & 2 & 23 & 898.1761 & -14 & 4 \\
\hline 23 & 1 & 22 & 24 & 1 & 23 & 898.1999 & -9 & 4 \\
\hline 23 & 4 & 19 & 24 & 4 & 20 & 898.2137 & -7 & 4 \\
\hline 23 & 2 & 21 & 24 & 2 & 22 & 898.2204 & -3 & 4 \\
\hline 23 & 5 & 18 & 24 & 5 & 19 & 898.2278 & 5 & 4 \\
\hline 23 & 6 & 17 & 24 & 6 & 18 & 898.2442 & 13 & 4 \\
\hline 23 & 7 & 16 & 24 & 7 & 17 & 898.2592 & 2 & 4 \\
\hline 23 & 8 & 15 & 24 & 8 & 16 & 898.2747 & 9 & 4 \\
\hline 23 & 9 & 14 & 24 & 9 & 15 & 898.2827 & -2 & 4 \\
\hline 22 & 0 & 22 & 23 & 0 & 23 & 899.9344 & -17 & 10 \\
\hline 22 & 1 & 22 & 23 & 1 & 23 & 899.9344 & 4 & 10 \\
\hline 22 & 2 & 21 & 23 & 2 & 22 & 899.9559 & -13 & 4 \\
\hline 22 & 1 & 21 & 23 & 1 & 22 & 899.9632 & -7 & 4 \\
\hline 22 & 2 & 20 & 23 & 2 & 21 & 899.9746 & 1 & 10 \\
\hline 22 & 4 & 18 & 23 & 4 & 19 & 899.9883 & -3 & 4 \\
\hline 22 & 5 & 17 & 23 & 5 & 18 & 900.0061 & 3 & 4 \\
\hline 22 & 6 & 16 & 23 & 6 & 17 & 900.0268 & 8 & 10 \\
\hline 22 & 7 & 15 & 23 & 7 & 16 & 900.0479 & 1 & 4 \\
\hline 22 & 8 & 14 & 23 & 8 & 15 & 900.0694 & -2 & 4 \\
\hline 22 & 9 & 13 & 23 & 9 & 14 & 900.0873 & -6 & 4 \\
\hline 21 & 1 & 20 & 22 & 1 & 21 & 901.7178 & 5 & 10 \\
\hline 21 & 1 & 21 & 22 & 1 & 22 & 901.7227 & -12 & 10 \\
\hline 21 & 2 & 19 & 22 & 2 & 20 & 901.7227 & 4 & 10 \\
\hline 21 & 3 & 18 & 22 & 3 & 19 & 901.7376 & 6 & 4 \\
\hline 21 & 4 & 17 & 22 & 4 & 18 & 901.7547 & 0 & 4 \\
\hline 21 & 5 & 16 & 22 & 5 & 17 & 901.7760 & 2 & 4 \\
\hline 21 & 6 & 15 & 22 & 6 & 16 & 901.8006 & 3 & 4 \\
\hline 21 & 7 & 14 & 22 & 7 & 15 & 901.8277 & 4 & 4 \\
\hline 21 & 8 & 13 & 22 & 8 & 14 & 901.8568 & 11 & 4 \\
\hline 20 & 1 & 19 & 21 & 1 & 20 & 903.4619 & 6 & 10 \\
\hline 20 & 2 & 19 & 21 & 2 & 20 & 903.4860 & -10 & 4 \\
\hline 20 & 3 & 18 & 21 & 3 & 19 & 903.4930 & -7 & 10 \\
\hline 20 & 0 & 20 & 21 & 0 & 21 & 903.4930 & -17 & 10 \\
\hline 20 & 1 & 20 & 21 & 1 & 21 & 903.5007 & -15 & 4 \\
\hline 20 & 4 & 16 & 21 & 4 & 17 & 903.5129 & 2 & 4 \\
\hline 20 & 5 & 15 & 21 & 5 & 16 & 903.5373 & 1 & 4 \\
\hline 20 & 6 & 14 & 21 & 6 & 15 & 903.5662 & 5 & 4 \\
\hline 20 & 7 & 13 & 21 & 7 & 14 & 903.5977 & 1 & 4 \\
\hline 20 & 8 & 12 & 21 & 8 & 13 & 903.6316 & -7 & 4 \\
\hline 20 & 9 & 11 & 21 & 9 & 12 & 903.6674 & -1 & 4 \\
\hline 19 & 1 & 18 & 20 & 1 & 19 & 905.1972 & 7 & 4 \\
\hline
\end{tabular}

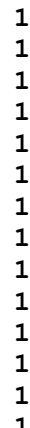

18

18

18

18

18

18
17

17

17

17

17

17
17

17

17

16

16
16

16

16

16

16
16

16

\begin{tabular}{|c|c|c|c|c|}
\hline 2 & 17 & 20 & 2 & 18 \\
\hline 2 & 18 & 20 & 2 & 19 \\
\hline 3 & 16 & 20 & 3 & 17 \\
\hline 0 & 19 & 20 & 0 & 20 \\
\hline 4 & 15 & 20 & 4 & 16 \\
\hline 1 & 19 & 20 & 1 & 20 \\
\hline 5 & 14 & 20 & 5 & 15 \\
\hline 6 & 13 & 20 & 6 & 14 \\
\hline 7 & 12 & 20 & 7 & 13 \\
\hline 8 & 11 & 20 & 8 & 12 \\
\hline 1 & 17 & 19 & 1 & 18 \\
\hline 2 & 16 & 19 & 2 & 17 \\
\hline 3 & 15 & 19 & 3 & 16 \\
\hline 3 & 16 & 19 & 3 & 17 \\
\hline 4 & 14 & 19 & 4 & 15 \\
\hline 1 & 18 & 19 & 1 & 19 \\
\hline 5 & 13 & 19 & 5 & 14 \\
\hline 6 & 12 & 19 & 6 & 13 \\
\hline 7 & 11 & 19 & 7 & 12 \\
\hline 8 & 10 & 19 & 8 & 11 \\
\hline 9 & 9 & 19 & 9 & 10 \\
\hline 1 & 16 & 18 & 1 & 17 \\
\hline 2 & 15 & 18 & 2 & 16 \\
\hline 3 & 14 & 18 & 3 & 15 \\
\hline 2 & 16 & 18 & 2 & 17 \\
\hline 0 & 17 & 18 & 0 & 18 \\
\hline 4 & 13 & 18 & 4 & 14 \\
\hline 5 & 12 & 18 & 5 & 13 \\
\hline 6 & 11 & 18 & 6 & 12 \\
\hline 7 & 10 & 18 & 7 & 11 \\
\hline 9 & 8 & 18 & 9 & 9 \\
\hline 1 & 15 & 17 & 1 & 16 \\
\hline 2 & 14 & 17 & 2 & 15 \\
\hline 2 & 15 & 17 & 2 & 16 \\
\hline 0 & 16 & 17 & 0 & 17 \\
\hline 4 & 12 & 17 & 4 & 13 \\
\hline 5 & 11 & 17 & 5 & 12 \\
\hline 1 & 16 & 17 & 1 & 17 \\
\hline 6 & 10 & 17 & 6 & 11 \\
\hline 7 & 9 & 17 & 7 & 10 \\
\hline 8 & 8 & 17 & 8 & 9 \\
\hline 9 & 7 & 17 & 9 & 8 \\
\hline 1 & 14 & 16 & 1 & 15 \\
\hline 2 & 13 & 16 & 2 & 14 \\
\hline 2 & 14 & 16 & 2 & 15 \\
\hline 3 & 13 & 16 & 3 & 14 \\
\hline 0 & 15 & 16 & 0 & 16 \\
\hline 4 & 11 & 16 & 4 & 12 \\
\hline 5 & 10 & 16 & 5 & 11 \\
\hline 1 & 15 & 16 & 1 & 16 \\
\hline 6 & 9 & 16 & 6 & 10 \\
\hline 7 & 8 & 16 & 7 & 9 \\
\hline 8 & 7 & 16 & 8 & 8 \\
\hline 9 & 6 & 16 & 9 & 7 \\
\hline 1 & 13 & 15 & 1 & 14 \\
\hline 2 & 12 & 15 & 2 & 13 \\
\hline 3 & 11 & 15 & 3 & 12 \\
\hline 2 & 13 & 15 & 2 & 14 \\
\hline 3 & 12 & 15 & 3 & 13 \\
\hline 0 & 14 & 15 & 0 & 15 \\
\hline 4 & 10 & 15 & 4 & 11 \\
\hline 5 & 9 & 15 & 5 & 10 \\
\hline 1 & 14 & 15 & 1 & 15 \\
\hline 6 & 8 & 15 & 6 & 9 \\
\hline 7 & 7 & 15 & 7 & 8 \\
\hline 8 & 6 & 15 & 8 & 7 \\
\hline 9 & 5 & 15 & 9 & 6 \\
\hline 1 & 12 & 14 & 1 & 13 \\
\hline 2 & 11 & 14 & 2 & 12 \\
\hline 2 & 12 & 14 & 2 & 13 \\
\hline 3 & 10 & 14 & 3 & 11 \\
\hline 0 & 13 & 14 & 0 & 14 \\
\hline 4 & 9 & 14 & 4 & 10 \\
\hline 5 & 8 & 14 & 5 & 9 \\
\hline 1 & 13 & 14 & 1 & 14 \\
\hline 6 & 7 & 14 & 6 & 8 \\
\hline 7 & 6 & 14 & 7 & 7 \\
\hline
\end{tabular}

$905.2012 \quad 7 \quad 10$ $\begin{array}{rrr}905.2367 & -7 & 4 \\ 905.2367 & 4 & 4\end{array}$ $905.2541 \quad-12 \quad 4$ $905.2626 \quad 2 \quad 4$ $905.2674 \quad-16 \quad 4$ $905.2902 \quad 2 \quad 4$ $\begin{array}{lll}905.3225 & 4 & 4\end{array}$ $905.3588 \quad 1 \quad 4$ $\begin{array}{rrr}905.3985 & -6 & 4 \\ 906.9240 & 9 & 4\end{array}$ $906.9324 \quad 14 \quad 4$ $906.9766 \quad 17 \quad 4$ $906.9800 \quad-4 \quad 10$ $907.0033 \quad-4 \quad 10$ $907.0224 \quad-21 \quad 4$ $907.0342 \quad 1 \quad 4$ $907.0696 \quad 0 \quad 4$ $907.1105 \quad 1 \quad 4$ $907.1560 \quad-2 \quad 4$

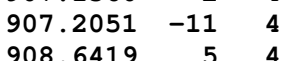
$908.6569 \quad 15 \quad 4$ $908.7063 \quad 7 \quad 10$ $908.7096 \quad-1 \quad 10$ $908.7375 \quad-25 \quad 4$ $908.7375 \quad 10 \quad 4$ $908.7684 \quad-9 \quad 4$ $\begin{array}{lll}908.8085 & 6 & 4 \\ 908.8530 & 3 & 4\end{array}$ $\begin{array}{rrr}908.8530 & 3 & 4 \\ 908.9600 & -1 & 10\end{array}$ $\begin{array}{rrr}908.9600 & -1 & 10 \\ 910.3531 & 14 & 4\end{array}$ $910.3750 \quad 13 \quad 4$ $910.4319 \quad 1 \quad 10$ $910.4618 \quad-27 \quad 10$ $910.4618 \quad 11 \quad 10$ $910.4963 \quad 6 \quad 10$ $910.5003 \quad-11 \quad 10$ $\begin{array}{lll}910.5370 & -2 & 4\end{array}$ $910.5854-2 \quad 4$ $910.6410 \quad-2 \quad 4$ $910.7037 \quad-2 \quad 4$ $912.0557 \quad 15 \quad 4$ $912.0868 \quad 12 \quad 4$ $912.1440 \quad-7 \quad 4$ $912.1475 \quad 3 \quad 10$ $912.1764 \quad-11 \quad 4$ $912.1764 \quad 2 \quad 4$ $912.2133 \quad 1 \quad 10$ $912.2214 \quad-18 \quad 4$ $912.2573 \quad 0 \quad 4$ $\begin{array}{rrr}912.3091 & 0 & 4 \\ 912.3689 & -1 & 4\end{array}$ $912.4369 \quad-5 \quad 4$ $913.7506 \quad 14 \quad 4$ $913.7921 \quad 14 \quad 4$ $\begin{array}{lll}913.8487 & 1 & 4 \\ 913.8487 & 4 & 4\end{array}$ $\begin{array}{lll}913.8531 & 7 & 10\end{array}$ $913.8789 \quad-5 \quad 10$ $913.8830 \quad 1 \quad 4$ $913.9217 \quad 1 \quad 4$ $\begin{array}{rrr}913.9321 & -18 & 4 \\ 913.9680 & -1 & 4\end{array}$ $914.0229-1 \quad 4$ $914.0868 \quad-2 \quad 4$ $914.1601 \quad-5 \quad 4$ $915.4381 \quad 14 \quad 4$ $915.4901 \quad 14 \quad 10$ $915.5434 \quad 5 \quad 10$ $\begin{array}{lll}915.5465 & 7 & 10\end{array}$ $\begin{array}{rrr}915.5700 & -7 & 4\end{array}$ $\begin{array}{rrr}915.6206 & -4 & 10\end{array}$ $\begin{array}{lll}915.6321 & -15 & 4\end{array}$ $915.6695 \quad-2 \quad 4$ $\begin{array}{rrr}915.7275 & 1 & 4\end{array}$ 


\begin{tabular}{|c|c|c|c|c|c|c|c|}
\hline 13 & 8 & 5 & 14 & 8 & 6 & 915.7949 & -2 \\
\hline 13 & 9 & 4 & 14 & 9 & 5 & 915.8739 & 4 \\
\hline 12 & 1 & 11 & 13 & 1 & 12 & 917.1184 & 15 \\
\hline 12 & 2 & 10 & 13 & 2 & 11 & 917.1799 & 8 \\
\hline 12 & 2 & 11 & 13 & 2 & 12 & 917.2283 & -1 \\
\hline 12 & 3 & 9 & 13 & 3 & 10 & 917.2356 & 14 \\
\hline 12 & 0 & 12 & 13 & 0 & 13 & 917.2513 & -4 \\
\hline 12 & 4 & 8 & 13 & 4 & 9 & 917.2697 & 1 \\
\hline 12 & 5 & 7 & 13 & 5 & 8 & 917.3112 & -1 \\
\hline 12 & 1 & 12 & 13 & 1 & 13 & 917.3212 & -12 \\
\hline 12 & 6 & 6 & 13 & 6 & 7 & 917.3618 & -1 \\
\hline 12 & 7 & 5 & 13 & 7 & 6 & 917.4221 & -2 \\
\hline 12 & 8 & 4 & 13 & 8 & 5 & 917.4932 & -1 \\
\hline 12 & 9 & 3 & 13 & 9 & 4 & 917.5771 & 10 \\
\hline 11 & 1 & 10 & 12 & 1 & 11 & 918.7911 & 12 \\
\hline 11 & 2 & 9 & 12 & 2 & 10 & 918.8621 & 6 \\
\hline 11 & 2 & 10 & 12 & 2 & 11 & 918.9049 & 0 \\
\hline 11 & 3 & 8 & 12 & 3 & 9 & 918.9148 & 10 \\
\hline 11 & 0 & 11 & 12 & 0 & 12 & 918.9225 & -4 \\
\hline 11 & 4 & 7 & 12 & 4 & 8 & 918.9494 & -1 \\
\hline 11 & 5 & 6 & 12 & 5 & 7 & 918.9924 & 0 \\
\hline 11 & 1 & 11 & 12 & 1 & 12 & 918.9993 & -12 \\
\hline 11 & 6 & 5 & 12 & 6 & 6 & 919.0446 & -2 \\
\hline 11 & 7 & 4 & 12 & 7 & 5 & 919.1073 & -2 \\
\hline 11 & 8 & 3 & 12 & 8 & 4 & 919.1813 & -2 \\
\hline 11 & 9 & 2 & 12 & 9 & 3 & 919.2683 & 0 \\
\hline 10 & 1 & 9 & 11 & 1 & 10 & 920.4569 & 12 \\
\hline 10 & 2 & 8 & 11 & 2 & 9 & 920.5360 & 5 \\
\hline 10 & 2 & 9 & 11 & 2 & 10 & 920.5726 & 1 \\
\hline 10 & 0 & 10 & 11 & 0 & 11 & 920.5848 & 1 \\
\hline 10 & 3 & 7 & 11 & 3 & 8 & 920.5848 & 5 \\
\hline 10 & 4 & 6 & 11 & 4 & 7 & 920.6203 & 1 \\
\hline 10 & 1 & 10 & 11 & 1 & 11 & 920.6651 & -27 \\
\hline 10 & 5 & 5 & 11 & 5 & 6 & 920.6651 & 8 \\
\hline 10 & 6 & 4 & 11 & 6 & 5 & 920.7182 & 0 \\
\hline 10 & 7 & 3 & 11 & 7 & 4 & 920.7827 & -3 \\
\hline 10 & 8 & 2 & 11 & 8 & 3 & 920.8600 & 2 \\
\hline 10 & 9 & 1 & 11 & 9 & 2 & 920.9505 & 3 \\
\hline 9 & 1 & 8 & 10 & 1 & 9 & 922.1153 & 9 \\
\hline 9 & 2 & 7 & 10 & 2 & 8 & 922.2009 & 2 \\
\hline 9 & 2 & 8 & 10 & 2 & 9 & 922.2309 & -1 \\
\hline 9 & 0 & 9 & 10 & 0 & 10 & 922.2379 & 5 \\
\hline 9 & 3 & 6 & 10 & 3 & 7 & 922.2461 & 4 \\
\hline 9 & 4 & 5 & 10 & 4 & 6 & 922.2817 & -1 \\
\hline 9 & 5 & 4 & 10 & 5 & 5 & 922.3251 & -17 \\
\hline 9 & 6 & 3 & 10 & 6 & 4 & 922.3822 & 0 \\
\hline 9 & 8 & 1 & 10 & 8 & 2 & 922.5292 & 11 \\
\hline 8 & 1 & 7 & 9 & 1 & 8 & 923.7667 & 8 \\
\hline 8 & 2 & 6 & 9 & 2 & 7 & 923.8569 & 2 \\
\hline 8 & 0 & 8 & 9 & 0 & 9 & 923.8809 & -4 \\
\hline 8 & 2 & 7 & 9 & 2 & 8 & 923.8809 & 2 \\
\hline 8 & 3 & 5 & 9 & 3 & 6 & 923.8981 & 2 \\
\hline 8 & 4 & 4 & 9 & 4 & 5 & 923.9341 & -2 \\
\hline 8 & 1 & 8 & 9 & 1 & 9 & 923.9696 & -8 \\
\hline 8 & 5 & 3 & 9 & 5 & 4 & 923.9798 & -3 \\
\hline 8 & 6 & 2 & 9 & 6 & 3 & 924.0365 & -1 \\
\hline 8 & 7 & 1 & 9 & 7 & 2 & 924.1049 & -1 \\
\hline 7 & 1 & 6 & 8 & 1 & 7 & 925.4111 & 7 \\
\hline 7 & 2 & 5 & 8 & 2 & 6 & 925.5032 & 0 \\
\hline 7 & 0 & 7 & 8 & 0 & 8 & 925.5166 & -2 \\
\hline 7 & 2 & 6 & 8 & 2 & 7 & 925.5219 & 6 \\
\hline 7 & 3 & 4 & 8 & 3 & 5 & 925.5410 & 1 \\
\hline 7 & 4 & 3 & 8 & 4 & 4 & 925.5772 & -2 \\
\hline 7 & 1 & 7 & 8 & 1 & 8 & 925.6050 & -7 \\
\hline 7 & 5 & 2 & 8 & 5 & 3 & 925.6236 & -3 \\
\hline 7 & 6 & 1 & 8 & 6 & 2 & 925.6815 & 0 \\
\hline 7 & 7 & 0 & 8 & 7 & 1 & 925.7511 & -2 \\
\hline 6 & 1 & 5 & 7 & 1 & 6 & 927.0482 & 5 \\
\hline 6 & 2 & 4 & 7 & 2 & 5 & 927.1402 & 1 \\
\hline 6 & 0 & 6 & 7 & 0 & 7 & 927.1438 & -1 \\
\hline 6 & 2 & 5 & 7 & 2 & 6 & 927.1529 & -1 \\
\hline 6 & 3 & 3 & 7 & 3 & 4 & 927.1745 & 0 \\
\hline 6 & 4 & 2 & 7 & 4 & 3 & 927.2110 & -2 \\
\hline 6 & 1 & 6 & 7 & 1 & 7 & 927.2301 & -4 \\
\hline 6 & 5 & 1 & 7 & 5 & 2 & 927.2583 & 0 \\
\hline 6 & 6 & 0 & 7 & 6 & 1 & 927.3169 & 1 \\
\hline 5 & 1 & 4 & 6 & 1 & 5 & 928.6782 & 3 \\
\hline
\end{tabular}

\begin{tabular}{|c|c|c|c|c|}
\hline 5 & 0 & 5 & 6 & 0 \\
\hline 5 & 2 & 3 & 6 & 2 \\
\hline 5 & 2 & 4 & 6 & 2 \\
\hline 5 & 3 & 2 & 6 & 3 \\
\hline 5 & 4 & 1 & 6 & 4 \\
\hline 5 & 1 & 5 & 6 & 1 \\
\hline 5 & 5 & 0 & 6 & 5 \\
\hline 4 & 1 & 3 & 5 & 1 \\
\hline 4 & 0 & 4 & 5 & 0 \\
\hline 4 & 2 & 2 & 5 & 2 \\
\hline 4 & 2 & 3 & 5 & 2 \\
\hline 4 & 3 & 1 & 5 & 3 \\
\hline 4 & 4 & 0 & 5 & 4 \\
\hline 4 & 1 & 4 & 5 & 1 \\
\hline 3 & 1 & 2 & 4 & 1 \\
\hline 3 & 0 & 3 & 4 & 0 \\
\hline 3 & 2 & 1 & 4 & 2 \\
\hline 3 & 3 & 0 & 4 & 3 \\
\hline 3 & 1 & 3 & 4 & 1 \\
\hline 2 & 1 & 1 & 3 & 1 \\
\hline 2 & 0 & 2 & 3 & 0 \\
\hline 2 & 1 & 2 & 3 & 1 \\
\hline 19 & 10 & 10 & 19 & 10 \\
\hline 18 & 7 & 12 & 18 & 7 \\
\hline 18 & 8 & 11 & 18 & 8 \\
\hline 18 & 9 & 10 & 18 & 9 \\
\hline 17 & 6 & 12 & 17 & 6 \\
\hline 18 & 10 & 9 & 18 & 10 \\
\hline 17 & 7 & 11 & 17 & 7 \\
\hline 17 & 8 & 10 & 17 & 8 \\
\hline 17 & 9 & 9 & 17 & 9 \\
\hline 16 & 5 & 12 & 16 & 5 \\
\hline 16 & 6 & 11 & 16 & 6 \\
\hline 16 & 7 & 10 & 16 & 7 \\
\hline 17 & 10 & 8 & 17 & 10 \\
\hline 16 & 8 & 9 & 16 & 8 \\
\hline 15 & 5 & 11 & 15 & 5 \\
\hline 16 & 9 & 8 & 16 & 9 \\
\hline 15 & 6 & 10 & 15 & 6 \\
\hline 15 & 7 & 9 & 15 & 7 \\
\hline 14 & 4 & 11 & 14 & 4 \\
\hline 15 & 8 & 8 & 15 & 8 \\
\hline 14 & 5 & 10 & 14 & 5 \\
\hline 14 & 6 & 9 & 14 & 6 \\
\hline 15 & 9 & 7 & 15 & 9 \\
\hline 14 & 7 & 8 & 14 & 7 \\
\hline 13 & 4 & 10 & 13 & 4 \\
\hline 13 & 5 & 9 & 13 & 5 \\
\hline 14 & 8 & 7 & 14 & 8 \\
\hline 13 & 6 & 8 & 13 & 6 \\
\hline 15 & 10 & 6 & 15 & 10 \\
\hline 14 & 9 & 6 & 14 & 9 \\
\hline 12 & 4 & 9 & 12 & 4 \\
\hline 13 & 7 & 7 & 13 & 7 \\
\hline 12 & 5 & 8 & 12 & 5 \\
\hline 13 & 8 & 6 & 13 & 8 \\
\hline 12 & 6 & 7 & 12 & 6 \\
\hline 11 & 3 & 9 & 11 & 3 \\
\hline 11 & 4 & 8 & 11 & 4 \\
\hline 12 & 7 & 6 & 12 & 7 \\
\hline 13 & 9 & 5 & 13 & 9 \\
\hline 11 & 5 & 7 & 11 & 5 \\
\hline 10 & 4 & 7 & 10 & 4 \\
\hline 11 & 7 & 5 & 11 & 7 \\
\hline 10 & 5 & 6 & 10 & 5 \\
\hline 12 & 9 & 4 & 12 & 9 \\
\hline 9 & 3 & 7 & 9 & 3 \\
\hline 10 & 6 & 5 & 10 & 6 \\
\hline 9 & 4 & 6 & 9 & 4 \\
\hline 11 & 8 & 4 & 11 & 8 \\
\hline 9 & 5 & 5 & 9 & 5 \\
\hline 8 & 3 & 6 & 8 & 3 \\
\hline 12 & 10 & 3 & 12 & 10 \\
\hline 10 & 7 & 4 & 10 & 7 \\
\hline 8 & 4 & 5 & 8 & 4 \\
\hline 9 & 6 & 4 & 9 & 6 \\
\hline 11 & 9 & 3 & 11 & 9 \\
\hline
\end{tabular}

\begin{tabular}{|c|c|c|}
\hline 8.1629 & & \\
\hline 8.7674 & & \\
\hline 28.7756 & -2 & \\
\hline 8.7987 & -2 & \\
\hline 8.8351 & -6 & \\
\hline 8.8443 & -4 & \\
\hline 28.8831 & -1 & \\
\hline 30.3011 & & \\
\hline 30.3736 & 1 & \\
\hline 30.3842 & 0 & \\
\hline 30.3889 & -6 & \\
\hline 30.4136 & -2 & \\
\hline 30.4488 & -19 & \\
\hline 30.4488 & 5 & \\
\hline 31.9167 & 1 & \\
\hline 931.9758 & -2 & \\
\hline 931.9926 & 12 & \\
\hline 32.0193 & 0 & \\
\hline 932.0412 & -1 & \\
\hline 933.5249 & -2 & \\
\hline 33.5699 & -2 & \\
\hline 933.6230 & -5 & \\
\hline 936.7999 & -11 & \\
\hline 36.8410 & -3 & \\
\hline 936.8795 & 6 & \\
\hline 936.9209 & 6 & \\
\hline 936.9857 & 3 & \\
\hline 937.0145 & 5 & \\
\hline 937.0230 & 5 & \\
\hline 937.0648 & -10 & \\
\hline 937.1138 & -6 & \\
\hline 937.1186 & 3 & \\
\hline 937.1527 & 4 & \\
\hline 937.1945 & 8 & \\
\hline 937.2185 & 12 & \\
\hline 937.2425 & 2 & \\
\hline 937.2723 & 2 & \\
\hline 937.2979 & 4 & \\
\hline 937.3094 & -1 & \\
\hline 937.3555 & 6 & \\
\hline 937.3851 & 3 & \\
\hline 937.4077 & -6 & \\
\hline 937.4175 & 10 & 10 \\
\hline 937.4572 & 3 & \\
\hline 937.4695 & -3 & \\
\hline 937.5062 & 1 & 10 \\
\hline 937.5177 & 5 & 10 \\
\hline 937.5511 & -3 & 10 \\
\hline 937.5640 & 0 & \\
\hline 937.5947 & 1 & \\
\hline 937.6060 & 14 & \\
\hline 937.6312 & 0 & \\
\hline 937.6409 & 5 & \\
\hline 937.6471 & -1 & \\
\hline 937.6766 & -1 & \\
\hline 937.7091 & -2 & \\
\hline 937.7221 & -5 & \\
\hline 937.7221 & 11 & \\
\hline 937.7541 & -2 & \\
\hline 937.77 & 5 & \\
\hline 937.7820 & 2 & \\
\hline 937.7929 & 3 & \\
\hline 937.8585 & -3 & \\
\hline 937.8990 & -3 & \\
\hline 937.8990 & 1 & \\
\hline 937.9218 & 2 & \\
\hline 937.9218 & 7 & \\
\hline 937.9491 & -1 & \\
\hline 937.9 & -4 & \\
\hline 937.9680 & -8 & \\
\hline 937.9955 & -1 & \\
\hline 938.0055 & -9 & \\
\hline 938.0055 & 4 & \\
\hline 938.0095 & -7 & \\
\hline 938.03 & 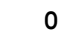 & \\
\hline 938.0482 & 4 & 10 \\
\hline 938.0513 & 8 & \\
\hline
\end{tabular}




\begin{tabular}{|c|c|}
\hline 38.0829 & 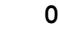 \\
\hline 938.0829 & 2 \\
\hline 938.1110 & -1 \\
\hline 938.1158 & -2 \\
\hline 938.1364 & -1 \\
\hline 938.1469 & -13 \\
\hline 938.1601 & -1 \\
\hline 938.1690 & 3 \\
\hline 938.1831 & 3 \\
\hline 938.1875 & 9 \\
\hline 938.2024 & -25 \\
\hline 938.2024 & 5 \\
\hline 938.2152 & -3 \\
\hline 938.2281 & 1 \\
\hline 938.2396 & -6 \\
\hline 938.2517 & -5 \\
\hline 938.2710 & 14 \\
\hline 941.3720 & 1 \\
\hline 941.4071 & -5 \\
\hline 942.8881 & 0 \\
\hline 942.9460 & 0 \\
\hline 942.9678 & -13 \\
\hline 943.0161 & -4 \\
\hline 944.3922 & -7 \\
\hline 944.4737 & 0 \\
\hline 944.4975 & 4 \\
\hline 944.5011 & 1 \\
\hline 944.5231 & 1 \\
\hline 944.5709 & 2 \\
\hline 945.8857 & -4 \\
\hline 945.9901 & 0 \\
\hline 946.0169 & 1 \\
\hline 946.0250 & 1 \\
\hline 946.0433 & -1 \\
\hline 946.0779 & 5 \\
\hline 946.1177 & 11 \\
\hline 947.3670 & -4 \\
\hline 947.4944 & -1 \\
\hline 947.5263 & -1 \\
\hline 947.5412 & 1 \\
\hline 947.5545 & 0 \\
\hline 947.5871 & -2 \\
\hline 947.6311 & 1 \\
\hline 947.6545 & 5 \\
\hline 948.8360 & -8 \\
\hline 948.9864 & 0 \\
\hline 949.0258 & -1 \\
\hline 949.0501 & -2 \\
\hline 949.0563 & 0 \\
\hline 949.0875 & -1 \\
\hline 949.1297 & -2 \\
\hline 949.1832 & 0 \\
\hline 949.1832 & 6 \\
\hline 950.2932 & -7 \\
\hline 950.4652 & 3 \\
\hline 950.5151 & 0 \\
\hline 950.5486 & -3 \\
\hline 950.5524 & -3 \\
\hline 950.5784 & 1 \\
\hline 950.6190 & 0 \\
\hline 950.6702 & -3 \\
\hline 950.7033 & 11 \\
\hline 950.7335 & 2 \\
\hline 951.7389 & 2 \\
\hline 951.9292 & -2 \\
\hline 951.9938 & 0 \\
\hline 952.0320 & -4 \\
\hline 952.0493 & 2 \\
\hline 952.0593 & -1 \\
\hline 952.0982 & -1 \\
\hline 952.1475 & -2 \\
\hline 952.2078 & -2 \\
\hline 952.2136 & 11 \\
\hline 953.1698 & -12 \\
\hline 953.3791 & -1 \\
\hline 953.4619 & 0 \\
\hline 953.5060 & -10 \\
\hline
\end{tabular}

$\begin{array}{rrrrrr}10 & 4 & 6 & 9 & 4 & 5 \\ 10 & 2 & 8 & 9 & 2 & 7 \\ 10 & 5 & 5 & 9 & 5 & 4 \\ 10 & 6 & 4 & 9 & 6 & 3 \\ 10 & 7 & 3 & 9 & 7 & 2 \\ 10 & 1 & 9 & 9 & 1 & 8 \\ 10 & 8 & 2 & 9 & 8 & 1 \\ 11 & 1 & 11 & 10 & 1 & 10\end{array}$

$953.5308 \quad-24$ $\begin{array}{rrr}953.5400 & 3 & 4 \\ 953.5676 & -1 & 4\end{array}$ $953.6145 \quad-3 \quad 4$ $953.6723-1 \quad 4$ $953.7144 \quad 11 \quad 4$ $953.7417 \quad 3 \quad 4$ $954.5892 \quad-12 \quad 4$ $954.9192 \quad 114$ $\begin{array}{lll}954.9699 & 8 & 10\end{array}$ 954.9730310 $\begin{array}{rrr}954.9931 & 1 & 4 \\ 955.0266 & -6 & 4\end{array}$ $955.0266 \quad 14 \quad 4$ $955.0717-1 \quad 4$ $955.1264 \quad 0 \quad 4$ $\begin{array}{rrr}955.1919 & 1 & 4 \\ 955.2055 & 13 & 4\end{array}$ $955.2698 \quad 7 \quad 10$ $955.9956-13 \quad 4$ $956.2318-4 \quad 4$ $956.3653 \quad 0 \quad 4$ $956.4243 \quad 2 \quad 4$ $956.4305 \quad 8 \quad 4$ 956.445622 $956.4769 \quad 0 \quad 4$ $956.5065 \quad 8 \quad 4$ $956.5187 \quad 1 \quad 4$ $956.5698 \quad-2 \quad 4$ $\begin{array}{lll}956.6313 & -1 & 4 \\ 956.6861 & 13 & 4\end{array}$ $956.7044 \quad 6 \quad 4$ $957.3891-11 \quad 4$ $957.6336 \quad-7 \quad 4$ $957.8002 \quad 0 \quad 4$ $957.8699 \quad 2 \quad 4$ $957.8787 \quad 3 \quad 4$ $\begin{array}{lll}957.8884 & 0 & 4 \\ 957.9169 & 1 & 4\end{array}$ $957.9553 \quad-1 \quad 4$ $957.9828 \quad 12 \quad 4$ $958.0030-1 \quad 4$ $958.0602-1 \quad 4$ $\begin{array}{lll}958.1272 & -1 & 4\end{array}$ $958.1544-3 \quad 4$ $958.7686-16 \quad 4$ $959.0189-6 \quad 4$ $959.2236-1 \quad 4$ $959.3069 \quad 10 \quad 4$ $959.3213 \quad-7 \quad 4$ $959.3213 \quad 25$ $\begin{array}{lll}959.3469 & 1 & 4\end{array}$ $959.3819 \quad 0 \quad 4$ $959.4257-1 \quad 4$ $959.4539 \quad 10 \quad 4$ $959.4781 \quad-1 \quad 4$ $959.5396 \quad 114$ $959.6150 \quad 15 \quad 4$ $\begin{array}{rrr}960.1351 & -17 & 4 \\ 960.3870 & -8 & 4\end{array}$ $960.6353-3 \quad 4$ $960.7330 \quad 4 \quad 4$ $960.7460 \quad-2 \quad 4$ $960.7519 \quad 5 \quad 4$ $\begin{array}{lll}960.7671 & 1 & 4 \\ 960.7983 & 0 & 4\end{array}$ $960.8379-1 \quad 4$ $960.9210 \quad 13 \quad 4$ $960.9401 \quad-3 \quad 4$ $961.0621 \quad 15 \quad 4$ $961.4878-18 \quad 4$ $961.7381 \quad-9 \quad 4$ $\begin{array}{rrr}962.0351 & -4 & 4\end{array}$ $962.1608 \quad-2 \quad 4$ $962.1772 \quad-1 \quad 4$ 


\begin{tabular}{|c|c|c|}
\hline 962.1772 & 8 & 4 \\
\hline 962.2045 & 0 & 4 \\
\hline 962.2394 & -2 & 4 \\
\hline 962.2812 & -4 & 10 \\
\hline 962.3299 & 0 & 4 \\
\hline 962.3830 & 14 & 4 \\
\hline 962.4971 & 15 & 4 \\
\hline 962.8268 & -18 & 4 \\
\hline 963.0722 & -10 & 4 \\
\hline 963.4229 & -4 & 4 \\
\hline 963.5578 & 3 & 4 \\
\hline 963.5663 & -3 & 4 \\
\hline 963.5780 & 2 & 4 \\
\hline 963.5946 & 4 & 4 \\
\hline 963.6009 & 4 & 4 \\
\hline 963.6306 & -1 & 4 \\
\hline 963.6663 & -6 & 4 \\
\hline 963.7073 & -7 & 4 \\
\hline 963.8397 & 15 & 4 \\
\hline 963.9190 & 12 & 4 \\
\hline 964.1519 & -18 & 4 \\
\hline 964.3894 & -13 & 4 \\
\hline 964.7983 & -4 & 4 \\
\hline 964.9555 & 2 & 4 \\
\hline 964.9623 & -6 & 4 \\
\hline 964.9689 & 3 & 4 \\
\hline 964.9866 & 3 & 4 \\
\hline 965.0057 & 4 & 4 \\
\hline 965.0113 & 1 & 4 \\
\hline 965.0408 & -4 & 4 \\
\hline 965.0743 & -3 & 4 \\
\hline 965.2903 & 12 & 4 \\
\hline 965.3276 & 11 & 4 \\
\hline 965.6903 & -13 & 4 \\
\hline 966.1609 & -6 & 4 \\
\hline 966.3435 & 1 & 4 \\
\hline 966.3496 & -6 & 10 \\
\hline 966.3496 & 1 & 10 \\
\hline 966.3621 & 2 & 4 \\
\hline 966.3806 & -5 & 4 \\
\hline 966.4041 & -3 & 4 \\
\hline 966.4107 & 6 & 4 \\
\hline 966.4284 & -12 & 4 \\
\hline 966.7217 & 6 & 4 \\
\hline 966.7350 & 14 & 4 \\
\hline 966.7599 & -15 & 4 \\
\hline 966.9752 & -13 & 4 \\
\hline 967.5105 & -9 & 4 \\
\hline 967.7212 & -1 & 4 \\
\hline 967.7212 & -35 & 4 \\
\hline 967.7212 & 5 & 4 \\
\hline 967.7277 & -9 & 10 \\
\hline 967.7277 & 4 & 10 \\
\hline 967.7405 & 1 & 4 \\
\hline 967.7566 & 0 & 4 \\
\hline 967.7722 & -8 & 4 \\
\hline 967.8093 & 3 & 4 \\
\hline 968.0427 & -11 & 4 \\
\hline 968.1013 & 5 & 4 \\
\hline 968.1722 & 13 & 4 \\
\hline 968.2449 & -7 & 4 \\
\hline 968.8473 & -9 & 4 \\
\hline 969.0826 & 1 & 4 \\
\hline 969.0826 & 5 & 4 \\
\hline 969.0894 & -30 & 4 \\
\hline 969.0894 & 4 & 4 \\
\hline 969.0980 & -1 & 4 \\
\hline 969.0980 & 3 & 4 \\
\hline 969.1043 & -4 & 4 \\
\hline 969.2031 & 4 & 4 \\
\hline 969.3114 & -5 & 4 \\
\hline 969.4650 & 0 & 4 \\
\hline 969.4991 & -4 & 4 \\
\hline 969.6011 & 9 & 4 \\
\hline 970.1705 & -11 & 4 \\
\hline 970.4275 & -2 & 10 \\
\hline 970.4275 & 0 & 10 \\
\hline
\end{tabular}

\begin{tabular}{|c|c|c|c|c|c|c|c|}
\hline 22 & 5 & 17 & 21 & 5 & 16 & 970.4343 & 5 \\
\hline 22 & 4 & 19 & 21 & 4 & 18 & 970.4506 & -3 \\
\hline 22 & 4 & 18 & 21 & 4 & 17 & 970.4589 & 0 \\
\hline 23 & 1 & 23 & 22 & 1 & 22 & 970.5658 & 4 \\
\hline 22 & 3 & 19 & 21 & 3 & 18 & 970.5915 & 0 \\
\hline 23 & 0 & 23 & 22 & 0 & 22 & 970.7386 & 3 \\
\hline 22 & 1 & 21 & 21 & 1 & 20 & 970.8122 & -4 \\
\hline 22 & 2 & 20 & 21 & 2 & 19 & 971.0211 & 4 \\
\hline 23 & 2 & 22 & 22 & 2 & 21 & 971.4804 & -9 \\
\hline 23 & 9 & 14 & 22 & 9 & 13 & 971.7320 & -10 \\
\hline 23 & 8 & 15 & 22 & 8 & 14 & 971.7467 & 2 \\
\hline 23 & 7 & 16 & 22 & 7 & 15 & 971.7547 & 4 \\
\hline 23 & 6 & 17 & 22 & 6 & 16 & 971.7625 & 3 \\
\hline 23 & 5 & 18 & 22 & 5 & 17 & 971.7759 & 0 \\
\hline 23 & 4 & 20 & 22 & 4 & 19 & 971.8000 & 0 \\
\hline 24 & 1 & 24 & 23 & 1 & 23 & 971.8055 & 11 \\
\hline 23 & 4 & 19 & 22 & 4 & 18 & 971.8120 & 9 \\
\hline 24 & 0 & 24 & 23 & 0 & 23 & 971.9635 & 9 \\
\hline 23 & 1 & 22 & 22 & 1 & 21 & 972.1417 & -14 \\
\hline 23 & 2 & 21 & 22 & 2 & 20 & 972.4314 & -1 \\
\hline 24 & 2 & 23 & 23 & 2 & 22 & 972.7760 & -12 \\
\hline 24 & 9 & 15 & 23 & 9 & 14 & 973.0303 & 10 \\
\hline 25 & 1 & 25 & 24 & 1 & 24 & 973.0303 & 16 \\
\hline 24 & 8 & 16 & 23 & 8 & 15 & 973.0545 & 4 \\
\hline 24 & 7 & 17 & 23 & 7 & 16 & 973.0717 & 8 \\
\hline 24 & 6 & 18 & 23 & 6 & 17 & 973.0872 & 4 \\
\hline 24 & 5 & 19 & 23 & 5 & 18 & 973.1085 & 2 \\
\hline 24 & 4 & 21 & 23 & 4 & 20 & 973.1401 & 2 \\
\hline 24 & 4 & 20 & 23 & 4 & 19 & 973.1550 & -1 \\
\hline 25 & 0 & 25 & 24 & 0 & 24 & 973.1741 & 16 \\
\hline 24 & 3 & 21 & 23 & 3 & 20 & 973.3564 & -3 \\
\hline 24 & 1 & 23 & 23 & 1 & 22 & 973.4535 & -21 \\
\hline 24 & 2 & 22 & 23 & 2 & 21 & 973.8311 & -8 \\
\hline 25 & 2 & 24 & 24 & 2 & 23 & 974.0576 & -14 \\
\hline 26 & 1 & 26 & 25 & 1 & 25 & 974.2418 & 35 \\
\hline 26 & 0 & 26 & 25 & 0 & 25 & 974.3714 & 32 \\
\hline 25 & 6 & 19 & 24 & 6 & 18 & 974.4015 & 4 \\
\hline 25 & 5 & 20 & 24 & 5 & 19 & 974.4308 & -4 \\
\hline 25 & 4 & 21 & 24 & 4 & 20 & 974.4908 & -2 \\
\hline 25 & 3 & 22 & 24 & 3 & 21 & 974.7334 & -6 \\
\hline 25 & 1 & 24 & 24 & 1 & 23 & 974.7472 & -22 \\
\hline 25 & 2 & 23 & 24 & 2 & 22 & 975.2192 & -19 \\
\hline 26 & 2 & 25 & 25 & 2 & 24 & 975.3252 & -12 \\
\hline 26 & 6 & 20 & 25 & 6 & 19 & 975.7061 & 9 \\
\hline 26 & 5 & 21 & 25 & 5 & 20 & 975.7441 & -4 \\
\hline 27 & 2 & 26 & 26 & 2 & 25 & 976.5777 & -14 \\
\hline 19 & 10 & 9 & 20 & 8 & 12 & 1020.5680 & -8 \\
\hline 18 & 10 & 8 & 19 & 8 & 11 & 1022.3585 & -8 \\
\hline 17 & 10 & 7 & 18 & 8 & 10 & 1024.1398 & 2 \\
\hline 16 & 10 & 6 & 17 & 8 & 9 & 1025.9117 & 3 \\
\hline 15 & 10 & 5 & 16 & 8 & 8 & 1027.6802 & 7 \\
\hline 14 & 10 & 4 & 15 & 8 & 7 & 1029.3071 & -8 \\
\hline 13 & 10 & 3 & 14 & 8 & 6 & 1031.0725 & -2 \\
\hline 17 & 10 & 8 & 17 & 8 & 9 & 1052.3017 & -1 \\
\hline 16 & 10 & 7 & 16 & 8 & 8 & 1052.5129 & 5 \\
\hline 15 & 10 & 6 & 15 & 8 & 7 & 1052.7198 & 10 \\
\hline 14 & 10 & 5 & 14 & 8 & 6 & 1052.7845 & -5 \\
\hline 13 & 10 & 4 & 13 & 8 & 5 & 1052.9868 & -2 \\
\hline 13 & 10 & 3 & 12 & 8 & 4 & 1073.3377 & -2 \\
\hline 14 & 10 & 4 & 13 & 8 & 5 & 1074.6984 & -8 \\
\hline 15 & 10 & 5 & 14 & 8 & 6 & 1076.1968 & 9 \\
\hline 16 & 10 & 6 & 15 & 8 & 7 & 1077.5524 & 6 \\
\hline 17 & 10 & 7 & 16 & 8 & 8 & 1078.9027 & -2 \\
\hline 18 & 10 & 8 & 17 & 8 & 9 & 1080.2436 & -6 \\
\hline 19 & 10 & 9 & 18 & 8 & 10 & 1081.5728 & -13 \\
\hline
\end{tabular}

$v_{8}$ Band

$\begin{array}{rrrrrrrrr}20 & 11 & 9 & 21 & 12 & 9 & 1186.4603 & -53 & 4 \\ 24 & 10 & 14 & 25 & 11 & 14 & 1187.7659 & -43 & 10 \\ 19 & 11 & 8 & 20 & 12 & 8 & 1187.9904 & -7 & 4 \\ 23 & 10 & 13 & 24 & 11 & 13 & 1189.2838 & -17 & 4 \\ 18 & 11 & 7 & 19 & 12 & 7 & 1189.5172 & 8 & 4 \\ 22 & 10 & 12 & 23 & 11 & 12 & 1190.8030 & 4 & 4 \\ 17 & 11 & 6 & 18 & 12 & 6 & 1191.0436 & 12 & 4 \\ 26 & 9 & 17 & 27 & 10 & 17 & 1191.9698 & -32 & 10 \\ 21 & 10 & 11 & 22 & 11 & 11 & 1192.3235 & 20 & 4\end{array}$




\begin{tabular}{|c|c|c|c|c|c|c|c|c|}
\hline 16 & 11 & 5 & 17 & 12 & 5 & 1192.5696 & 2 & 4 \\
\hline 25 & 9 & 16 & 26 & 10 & 16 & 1193.4879 & -28 & 10 \\
\hline 20 & 10 & 10 & 21 & 11 & 10 & 1193.8448 & 25 & 4 \\
\hline 29 & 8 & 21 & 30 & 9 & 21 & 1194.5420 & -54 & 4 \\
\hline 24 & 9 & 15 & 25 & 10 & 15 & 1195.0103 & 2 & 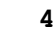 \\
\hline 19 & 10 & 9 & 20 & 11 & 9 & 1195.3680 & 29 & \\
\hline 14 & 11 & 3 & 15 & 12 & 3 & 1195.6323 & 29 & \\
\hline 28 & 8 & 20 & 29 & 9 & 20 & 1196.0641 & -37 & 10 \\
\hline 23 & 9 & 14 & 24 & 10 & 14 & 1196.5321 & 9 & 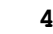 \\
\hline 18 & 10 & 8 & 19 & 11 & 8 & 1196.8927 & 27 & \\
\hline 13 & 11 & 2 & 14 & 12 & 2 & 1197.1612 & -3 & \\
\hline 27 & 8 & 19 & 28 & 9 & 19 & 1197.5878 & -19 & \\
\hline 22 & 9 & 13 & 23 & 10 & 13 & 1198.0554 & 13 & \\
\hline 17 & 10 & 7 & 18 & 11 & 7 & 1198.4192 & 24 & \\
\hline 31 & 7 & 24 & 32 & 8 & 24 & 1198.5276 & -38 & \\
\hline 12 & 11 & 1 & 13 & 12 & 1 & 1198.6936 & -23 & \\
\hline 26 & 8 & 18 & 27 & 9 & 18 & 1199.1112 & -17 & 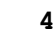 \\
\hline 21 & 9 & 12 & 22 & 10 & 12 & 1199.5807 & 19 & \\
\hline 16 & 10 & 6 & 17 & 11 & 6 & 1199.9475 & 17 & \\
\hline 30 & 7 & 23 & 31 & 8 & 23 & 1200.0550 & -24 & 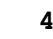 \\
\hline 11 & 11 & 0 & 12 & 12 & 0 & 1200.2281 & -44 & \\
\hline 25 & 8 & 17 & 26 & 9 & 17 & 1200.6377 & 2 & \\
\hline 20 & 9 & 11 & 21 & 10 & 11 & 1201.1071 & 18 & \\
\hline 15 & 10 & 5 & 16 & 11 & 5 & 1201.4779 & 11 & 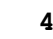 \\
\hline 29 & 7 & 22 & 30 & 8 & 22 & 1201.5833 & -10 & \\
\hline 24 & 8 & 16 & 25 & 9 & 16 & 1202.1643 & 7 & \\
\hline 19 & 9 & 10 & 20 & 10 & 10 & 1202.6355 & 18 & \\
\hline 14 & 10 & 4 & 15 & 11 & 4 & 1203.0101 & 1 & \\
\hline 28 & 7 & 21 & 29 & 8 & 21 & 1203.1111 & -10 & \\
\hline 23 & 8 & 15 & 24 & 9 & 15 & 1203.6921 & 8 & 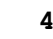 \\
\hline 18 & 9 & 9 & 19 & 10 & 9 & 1204.1657 & 19 & 4 \\
\hline 13 & 10 & 3 & 14 & 11 & 3 & 1204.5446 & -8 & \\
\hline 27 & 7 & 20 & 28 & 8 & 20 & 1204.6402 & -9 & \\
\hline 22 & 8 & 14 & 23 & 9 & 14 & 1205.2215 & 11 & 4 \\
\hline 17 & 9 & 8 & 18 & 10 & 8 & 1205.6973 & 14 & \\
\hline 12 & 10 & 2 & 13 & 11 & 2 & 1206.0812 & -17 & \\
\hline 26 & 7 & 19 & 27 & 8 & 19 & 1206.1715 & 4 & 4 \\
\hline 21 & 8 & 13 & 22 & 9 & 13 & 1206.7524 & 12 & 4 \\
\hline 16 & 9 & 7 & 17 & 10 & 7 & 1207.2308 & 9 & \\
\hline 11 & 10 & 1 & 12 & 11 & 1 & 1207.6200 & -26 & 4 \\
\hline 25 & 7 & 18 & 26 & 8 & 18 & 1207.7029 & 5 & 4 \\
\hline 20 & 8 & 12 & 21 & 9 & 12 & 1208.2847 & 12 & \\
\hline 29 & 6 & 23 & 30 & 7 & 23 & 1208.5511 & 0 & 4 \\
\hline 15 & 9 & 6 & 16 & 10 & 6 & 1208.7664 & 7 & 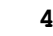 \\
\hline 10 & 10 & 0 & 11 & 11 & 0 & 1209.1611 & -34 & \\
\hline 24 & 7 & 17 & 25 & 8 & 17 & 1209.2352 & 4 & 4 \\
\hline 19 & 8 & 11 & 20 & 9 & 11 & 1209.8189 & 15 & 4 \\
\hline 28 & 6 & 22 & 29 & 7 & 22 & 1210.0854 & 11 & 4 \\
\hline 14 & 9 & 5 & 15 & 10 & 5 & 1210.3035 & 0 & \\
\hline 23 & 7 & 16 & 24 & 8 & 16 & 1210.7695 & 9 & 4 \\
\hline 18 & 8 & 10 & 19 & 9 & 10 & 1211.3540 & 10 & 4 \\
\hline 27 & 6 & 21 & 28 & 7 & 21 & 1211.6199 & 10 & \\
\hline 13 & 9 & 4 & 14 & 10 & 4 & 1211.8425 & -7 & 4 \\
\hline 22 & 7 & 15 & 23 & 8 & 15 & 1212.3041 & 5 & 4 \\
\hline 17 & 8 & 9 & 18 & 9 & 9 & 1212.8909 & 8 & \\
\hline 26 & 6 & 20 & 27 & 7 & 20 & 1213.1564 & 8 & 4 \\
\hline 26 & 6 & 21 & 27 & 7 & 21 & 1213.1618 & 5 & 4 \\
\hline 12 & 9 & 3 & 13 & 10 & 3 & 1213.3836 & -11 & \\
\hline 21 & 7 & 14 & 22 & 8 & 14 & 1213.8410 & 10 & 4 \\
\hline 30 & 5 & 25 & 31 & 6 & 25 & 1213.8864 & -27 & 4 \\
\hline 30 & 5 & 26 & 31 & 6 & 26 & 1213.8953 & -14 & 4 \\
\hline 16 & 8 & 8 & 17 & 9 & 8 & 1214.4295 & 6 & 4 \\
\hline 25 & 6 & 20 & 26 & 7 & 20 & 1214.6771 & -6 & 4 \\
\hline 25 & 6 & 19 & 26 & 7 & 19 & 1214.7055 & 4 & 4 \\
\hline 11 & 9 & 2 & 12 & 10 & 2 & 1214.9267 & -16 & 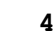 \\
\hline 20 & 7 & 13 & 21 & 8 & 13 & 1215.3785 & 9 & 4 \\
\hline 29 & 5 & 24 & 30 & 6 & 24 & 1215.4233 & -28 & 4 \\
\hline 29 & 5 & 25 & 30 & 6 & 25 & 1215.4332 & -2 & . \\
\hline 15 & 8 & 7 & 16 & 9 & 7 & 1215.9695 & 1 & 4 \\
\hline 24 & 6 & 18 & 25 & 7 & 18 & 1216.2218 & 5 & 4 \\
\hline 10 & 9 & 1 & 11 & 10 & 1 & 1216.4716 & -21 & 4 \\
\hline 19 & 7 & 12 & 20 & 8 & 12 & 1216.9173 & 6 & 4 \\
\hline 28 & 5 & 24 & 29 & 6 & 24 & 1216.9702 & -8 & 4 \\
\hline 14 & 8 & 6 & 15 & 9 & 6 & 1217.5117 & 2 & 4 \\
\hline 23 & 6 & 17 & 24 & 7 & 17 & 1217.7627 & 3 & \\
\hline 9 & 9 & 0 & 10 & 10 & 0 & 1218.0185 & -25 & 4 \\
\hline 18 & 7 & 11 & 19 & 8 & 11 & 1218.4577 & 6 & \\
\hline
\end{tabular}

\begin{tabular}{|c|c|c|c|c|c|c|c|c|}
\hline 27 & 5 & 22 & 28 & 6 & 22 & 1218.5011 & -18 & 4 \\
\hline 27 & 5 & 23 & 28 & 6 & 23 & 1218.5101 & 4 & 4 \\
\hline 12 & 11 & 1 & 12 & 12 & 1 & 1219.0195 & -26 & 10 \\
\hline 13 & 11 & 2 & 13 & 12 & 2 & 1219.0481 & -9 & 10 \\
\hline 13 & 8 & 5 & 14 & 9 & 5 & 1219.0551 & -2 & \\
\hline 14 & 11 & 3 & 14 & 12 & 3 & 1219.0799 & 23 & \\
\hline 16 & 11 & 5 & 16 & 12 & 5 & 1219.1376 & 4 & \\
\hline 17 & 11 & 6 & 17 & 12 & 6 & 1219.1710 & 18 & \\
\hline 18 & 11 & 7 & 18 & 12 & 7 & 1219.2019 & 5 & 10 \\
\hline 22 & 6 & 16 & 23 & 7 & 16 & 1219.3032 & 3 & \\
\hline 17 & 7 & 10 & 18 & 8 & 10 & 1219.9993 & 3 & \\
\hline 26 & 5 & 21 & 27 & 6 & 21 & 1220.0415 & -11 & \\
\hline 26 & 5 & 22 & 27 & 6 & 22 & 1220.0497 & 4 & \\
\hline 12 & 8 & 4 & 13 & 9 & 4 & 1220.6001 & -7 & 0 \\
\hline 21 & 6 & 15 & 22 & 7 & 15 & 1220.8447 & 5 & \\
\hline 16 & 7 & 9 & 17 & 8 & 9 & 1221.5425 & 3 & \\
\hline 25 & 5 & 20 & 26 & 6 & 20 & 1221.5830 & -2 & \\
\hline 25 & 5 & 21 & 26 & 6 & 21 & 1221.5905 & 5 & \\
\hline 11 & 8 & 3 & 12 & 9 & 3 & 1222.1470 & -9 & \\
\hline 29 & 4 & 25 & 30 & 5 & 25 & 1222.2717 & 5 & \\
\hline 20 & 6 & 14 & 21 & 7 & 14 & 1222.3867 & 1 & \\
\hline 15 & 7 & 8 & 16 & 8 & 8 & 1223.0869 & 1 & \\
\hline 24 & 5 & 19 & 25 & 6 & 19 & 1223.1249 & 6 & \\
\hline 24 & 5 & 20 & 25 & 6 & 20 & 1223.1322 & 5 & \\
\hline 10 & 8 & 2 & 11 & 9 & 2 & 1223.6954 & -13 & \\
\hline 28 & 4 & 24 & 29 & 5 & 24 & 1223.8072 & 20 & \\
\hline 19 & 6 & 13 & 20 & 7 & 13 & 1223.9304 & 2 & \\
\hline 14 & 7 & 7 & 15 & 8 & 7 & 1224.6329 & 0 & \\
\hline 23 & 5 & 18 & 24 & 6 & 18 & 1224.6659 & 4 & \\
\hline 23 & 5 & 19 & 24 & 6 & 19 & 1224.6749 & 4 & \\
\hline 9 & 8 & 1 & 10 & 9 & 1 & 1225.2457 & -14 & \\
\hline 27 & 4 & 24 & 28 & 5 & 24 & 1225.3140 & 19 & \\
\hline 27 & 4 & 23 & 28 & 5 & 23 & 1225.3420 & 5 & \\
\hline 18 & 6 & 12 & 19 & 7 & 12 & 1225.4752 & 3 & \\
\hline 13 & 7 & 6 & 14 & 8 & 6 & 1226.1803 & -1 & \\
\hline 22 & 5 & 17 & 23 & 6 & 17 & 1226.2042 & 7 & \\
\hline 22 & 5 & 18 & 23 & 6 & 18 & 1226.2185 & 1 & \\
\hline 11 & 10 & 1 & 11 & 11 & 1 & 1226.3899 & -36 & 10 \\
\hline 12 & 10 & 2 & 12 & 11 & 2 & 1226.4143 & -18 & \\
\hline 13 & 10 & 3 & 13 & 11 & 3 & 1226.4393 & -11 & \\
\hline 14 & 10 & 4 & 14 & 11 & 4 & 1226.4662 & -1 & \\
\hline 15 & 10 & 5 & 15 & 11 & 5 & 1226.4948 & 10 & \\
\hline 16 & 10 & 6 & 16 & 11 & 6 & 1226.5242 & 14 & \\
\hline 17 & 10 & 7 & 17 & 11 & 7 & 1226.5557 & 23 & \\
\hline 18 & 10 & 8 & 18 & 11 & 8 & 1226.5886 & 32 & \\
\hline 19 & 10 & 9 & 19 & 11 & 9 & 1226.6216 & 29 & \\
\hline 20 & 10 & 10 & 20 & 11 & 10 & 1226.6564 & 31 & \\
\hline 21 & 10 & 11 & 21 & 11 & 11 & 1226.6914 & 23 & \\
\hline 22 & 10 & 12 & 22 & 11 & 12 & 1226.7267 & 7 & \\
\hline 23 & 10 & 13 & 23 & 11 & 13 & 1226.7616 & -24 & \\
\hline 8 & 8 & 0 & 9 & 9 & 0 & 1226.7975 & -17 & \\
\hline 26 & 4 & 23 & 27 & 5 & 23 & 1226.8620 & 14 & \\
\hline 26 & 4 & 22 & 27 & 5 & 22 & 1226.8811 & 12 & \\
\hline 17 & 6 & 11 & 18 & 7 & 11 & 1227.0210 & 3 & \\
\hline 30 & 3 & 28 & 31 & 4 & 28 & 1227.1789 & -3 & \\
\hline 12 & 7 & 5 & 13 & 8 & 5 & 1227.7291 & -3 & \\
\hline 21 & 5 & 17 & 22 & 6 & 17 & 1227.7634 & 1 & \\
\hline 21 & 5 & 16 & 22 & 6 & 16 & 1227.7895 & -11 & \\
\hline 30 & 3 & 27 & 31 & 4 & 27 & 1227.9290 & 30 & \\
\hline 25 & 4 & 22 & 26 & 5 & 22 & 1228.4072 & 6 & \\
\hline 25 & 4 & 21 & 26 & 5 & 21 & 1228.4218 & 15 & \\
\hline 16 & 6 & 10 & 17 & 7 & 10 & 1228.5679 & 0 & \\
\hline 29 & 3 & 27 & 30 & 4 & 27 & 1228.7630 & -7 & \\
\hline 11 & 7 & 4 & 12 & 8 & 4 & 1229.2794 & -3 & \\
\hline 20 & 5 & 16 & 21 & 6 & 16 & 1229.3095 & 2 & \\
\hline 20 & 5 & 15 & 21 & 6 & 15 & 1229.3147 & -5 & \\
\hline 29 & 3 & 26 & 30 & 4 & 26 & 1229.3941 & 31 & \\
\hline 24 & 4 & 21 & 25 & 5 & 21 & 1229.9525 & -1 & \\
\hline 24 & 4 & 20 & 25 & 5 & 20 & 1229.9622 & -1 & \\
\hline 15 & 6 & 9 & 16 & 7 & 9 & 1230.1162 & 0 & \\
\hline 28 & 3 & 26 & 29 & 4 & 26 & 1230.3420 & -13 & \\
\hline 10 & 7 & 3 & 11 & 8 & 3 & 1230.8308 & -7 & \\
\hline 19 & 5 & 14 & 20 & 6 & 14 & 1230.8573 & -15 & \\
\hline 23 & 4 & 20 & 24 & 5 & 20 & 1231.4993 & 2 & \\
\hline 23 & 4 & 19 & 24 & 5 & 19 & 1231.5058 & -3 & \\
\hline 14 & 6 & 8 & 15 & 7 & 8 & 1231.6657 & 0 & \\
\hline 27 & 3 & 25 & 28 & 4 & 25 & 1231.9180 & -6 & \\
\hline
\end{tabular}




\begin{tabular}{|c|c|c|c|c|c|c|c|}
\hline 30 & 2 & 29 & 31 & 3 & 29 & 1232.1853 & -12 \\
\hline 27 & 3 & 24 & 28 & 4 & 24 & 1232.3503 & 11 \\
\hline 9 & 7 & 2 & 10 & 8 & 2 & 1232.3839 & -8 \\
\hline 18 & 5 & 13 & 19 & 6 & 13 & 1232.4051 & -7 \\
\hline 22 & 4 & 19 & 23 & 5 & 19 & 1233.0464 & 1 \\
\hline 22 & 4 & 18 & 23 & 5 & 18 & 1233.0518 & 6 \\
\hline 13 & 6 & 7 & 14 & 7 & 7 & 1233.2165 & 0 \\
\hline 26 & 3 & 24 & 27 & 4 & 24 & 1233.4897 & -5 \\
\hline 10 & 9 & 1 & 10 & 10 & 1 & 1233.6854 & -19 \\
\hline 11 & 9 & 2 & 11 & 10 & 2 & 1233.7031 & -20 \\
\hline 12 & 9 & 3 & 12 & 10 & 3 & 1233.7235 & -9 \\
\hline 13 & 9 & 4 & 13 & 10 & 4 & 1233.7447 & -4 \\
\hline 14 & 9 & 5 & 14 & 10 & 5 & 1233.7672 & 0 \\
\hline 15 & 9 & 6 & 15 & 10 & 6 & 1233.7912 & 5 \\
\hline 16 & 9 & 7 & 16 & 10 & 7 & 1233.8162 & 7 \\
\hline 26 & 3 & 23 & 27 & 4 & 23 & 1233.8428 & 10 \\
\hline 17 & 9 & 8 & 17 & 10 & 8 & 1233.8428 & 12 \\
\hline 18 & 9 & 9 & 18 & 10 & 9 & 1233.8708 & 18 \\
\hline 19 & 9 & 10 & 19 & 10 & 10 & 1233.8993 & 18 \\
\hline 29 & 2 & 28 & 30 & 3 & 28 & 1233.9206 & -13 \\
\hline 20 & 9 & 11 & 20 & 10 & 11 & 1233.9291 & 19 \\
\hline 8 & 7 & 1 & 9 & 8 & 1 & 1233.9385 & -8 \\
\hline 17 & 5 & 12 & 18 & 6 & 12 & 1233.9540 & -5 \\
\hline 21 & 9 & 12 & 21 & 10 & 12 & 1233.9597 & 18 \\
\hline 22 & 9 & 13 & 22 & 10 & 13 & 1233.9909 & 12 \\
\hline 23 & 9 & 14 & 23 & 10 & 14 & 1234.0228 & 4 \\
\hline 24 & 9 & 15 & 24 & 10 & 15 & 1234.0554 & -6 \\
\hline 25 & 9 & 16 & 25 & 10 & 16 & 1234.0889 & -15 \\
\hline 26 & 9 & 17 & 26 & 10 & 17 & 1234.1226 & -30 \\
\hline 27 & 9 & 18 & 27 & 10 & 18 & 1234.1568 & -46 \\
\hline 21 & 4 & 18 & 22 & 5 & 18 & 1234.5942 & -3 \\
\hline 21 & 4 & 17 & 22 & 5 & 17 & 1234.5982 & 3 \\
\hline 12 & 6 & 6 & 13 & 7 & 6 & 1234.7684 & -1 \\
\hline 25 & 3 & 23 & 26 & 4 & 23 & 1235.0580 & -5 \\
\hline 32 & 1 & 31 & 33 & 2 & 31 & 1235.2892 & 5 \\
\hline 25 & 3 & 22 & 26 & 4 & 22 & 1235.3427 & 1 \\
\hline 7 & 7 & 0 & 8 & 8 & 0 & 1235.4943 & -10 \\
\hline 16 & 5 & 11 & 17 & 6 & 11 & 1235.5042 & -3 \\
\hline 28 & 2 & 27 & 29 & 3 & 27 & 1235.6469 & 5 \\
\hline 30 & 2 & 28 & 31 & 3 & 28 & 1235.7557 & 22 \\
\hline 20 & 4 & 16 & 21 & 5 & 16 & 1236.1446 & -12 \\
\hline 11 & 6 & 5 & 12 & 7 & 5 & 1236.3216 & -1 \\
\hline 24 & 3 & 22 & 25 & 4 & 22 & 1236.6232 & -8 \\
\hline 24 & 3 & 21 & 25 & 4 & 21 & 1236.8512 & 1 \\
\hline 15 & 5 & 10 & 16 & 6 & 10 & 1237.0556 & -2 \\
\hline 29 & 2 & 27 & 30 & 3 & 27 & 1237.2403 & 26 \\
\hline 27 & 2 & 26 & 28 & 3 & 26 & 1237.3604 & 6 \\
\hline 19 & 4 & 15 & 20 & 5 & 15 & 1237.6941 & -9 \\
\hline 10 & 6 & 4 & 11 & 7 & 4 & 1237.8758 & -3 \\
\hline 28 & 1 & 28 & 29 & 2 & 28 & 1238.1372 & 10 \\
\hline 23 & 3 & 21 & 24 & 4 & 21 & 1238.1866 & -5 \\
\hline 23 & 3 & 20 & 24 & 4 & 20 & 1238.3666 & 0 \\
\hline 14 & 5 & 9 & 15 & 6 & 9 & 1238.6081 & -1 \\
\hline 28 & 2 & 26 & 29 & 3 & 26 & 1238.7081 & 20 \\
\hline 29 & 0 & 29 & 30 & 1 & 29 & 1238.8652 & 5 \\
\hline 26 & 2 & 25 & 27 & 3 & 25 & 1239.0618 & -5 \\
\hline 30 & 1 & 29 & 31 & 2 & 29 & 1239.1680 & 10 \\
\hline 18 & 4 & 14 & 19 & 5 & 14 & 1239.2445 & -9 \\
\hline 9 & 6 & 3 & 10 & 7 & 3 & 1239.4314 & -4 \\
\hline 22 & 3 & 20 & 23 & 4 & 20 & 1239.7480 & -4 \\
\hline 22 & 3 & 19 & 23 & 4 & 19 & 1239.8885 & 1 \\
\hline 27 & 1 & 27 & 28 & 2 & 27 & 1240.0338 & -10 \\
\hline 13 & 5 & 8 & 14 & 6 & 8 & 1240.1617 & 0 \\
\hline 25 & 2 & 24 & 26 & 3 & 24 & 1240.7532 & -7 \\
\hline 17 & 4 & 13 & 18 & 5 & 13 & 1240.7967 & -3 \\
\hline 9 & 8 & 1 & 9 & 9 & 1 & 1240.9001 & -15 \\
\hline 10 & 8 & 2 & 10 & 9 & 2 & 1240.9143 & -9 \\
\hline 11 & 8 & 3 & 11 & 9 & 3 & 1240.9294 & -7 \\
\hline 12 & 8 & 4 & 12 & 9 & 4 & 1240.9456 & -7 \\
\hline 13 & 8 & 5 & 13 & 9 & 5 & 1240.9633 & -4 \\
\hline 14 & 8 & 6 & 14 & 9 & 6 & 1240.9823 & 1 \\
\hline 8 & 6 & 2 & 9 & 7 & 2 & 1240.9884 & -2 \\
\hline 15 & 8 & 7 & 15 & 9 & 7 & 1241.0019 & 1 \\
\hline 16 & 8 & 8 & 16 & 9 & 8 & 1241.0232 & 6 \\
\hline 17 & 8 & 9 & 17 & 9 & 9 & 1241.0451 & 8 \\
\hline 18 & 8 & 10 & 18 & 9 & 10 & 1241.0680 & 9 \\
\hline 19 & 8 & 11 & 19 & 9 & 11 & 1241.0921 & 12 \\
\hline
\end{tabular}

$\begin{array}{rrr}1241.1169 & 13 & 4 \\ 1241.1425 & 14 & 4 \\ 1241.1683 & 9 & 4 \\ 1241.1954 & 10 & 4 \\ 1241.2225 & 3 & 4 \\ 1241.2505 & -1 & 4 \\ 1241.2785 & -10 & 4\end{array}$

$\begin{array}{lll}1241.3074 & -8 & 10\end{array}$

$1241.3074-14 \quad 10$

$\begin{array}{lll}1241.3352 & -34 & 4 \\ 1241.3635 & -52 & 4\end{array}$

$1241.4163 \quad 2 \quad 4$

$\begin{array}{lll}1241.6078 & 11 & 4 \\ 1241.7162 & -1 & 4\end{array}$

$\begin{array}{rrr}1241.9277 & 7 & 4\end{array}$

$1242.3492 \quad-4 \quad 4$

$1242.4346 \quad-4 \quad 4$

$1242.5461 \quad-5 \quad 4$

$1242.8666 \quad-3 \quad 4$

$1242.9342 \quad-2 \quad 4$

$\begin{array}{lll}1242.9484 & -4 & 4 \\ 1243.0460 & 12 & 4\end{array}$

$1243.2719 \quad 0 \quad 4$

$1243.8137 \quad 16 \quad 4$

$1243.9029 \quad-4 \quad 4$

$1244.1052 \quad-6 \quad 4$

$1244.4244 \quad-4 \quad 4$

$1244.4858 \quad-2 \quad 4$

$1244.7755 \quad-2 \quad 4$

$1244.8284 \quad-2 \quad 4$

$\begin{array}{lll}1245.2617 & -6 & 4 \\ 1245.4579 & -2 & 4\end{array}$

$\begin{array}{lll}1245.6893 & -1 & 4\end{array}$

$1245.7652 \quad-10 \quad 4$

$1245.9110 \quad-3 \quad 4$

$1245.9819 \quad-4 \quad 4$

$1246.0270 \quad-2 \quad 4$

$1246.3862-1 \quad 4$

$\begin{array}{lll}1246.5881 & -7 & 4 \\ 1247.0137 & -1 & 4\end{array}$

$1247.3450 \quad-2 \quad 4$

$\begin{array}{lll}1247.3853 & 7 & 4\end{array}$

$\begin{array}{lll}1247.4165 & -5 & 4\end{array}$

$\begin{array}{lll}1247.5391 & -4 & 4 \\ 1247.5579 & -6 & 4\end{array}$

$1247.5716 \quad-3 \quad 4$

$1247.9449 \quad-2 \quad 4$

$1248.0321-8 \quad 4$

$\begin{array}{lll}1248.0425 & -7 & 4\end{array}$

$1248.0539 \quad-6 \quad 4$

$1248.0664 \quad-5 \quad 4$

$\begin{array}{lll}1248.0799 & -4 & 4\end{array}$

$1248.0946 \quad-1 \quad 4$

$\begin{array}{rrr}1248.1099 & -1 & 4 \\ 1248.1264 & 2 & 4\end{array}$

$\begin{array}{lll}1248.1433 & 1 & 4\end{array}$

$\begin{array}{lll}1248.1616 & 4 & 4\end{array}$

$1248.1806 \quad 7 \quad 4$

$1248.2202 \quad 8 \quad 4$

$1248.2410 \quad 8 \quad 4$

$1248.2838 \quad 4 \quad 4$

1248.305924

$\begin{array}{rrr}1248.3288 & 4 & 4 \\ 1248.3514 & -1 & 4\end{array}$

$\begin{array}{lll}1248.3735 & -2 & 10\end{array}$

$1248.3735 \quad-13 \quad 10$

$\begin{array}{lll}1248.3964 & -19 & 4\end{array}$

$\begin{array}{rrr}1248.4418 & -34 & 4\end{array}$

$\begin{array}{lll}1248.4646 & -39 & 4\end{array}$

$1248.4847 \quad-68 \quad 4$

$\begin{array}{lll}1248.5066 & -73 & 4\end{array}$

$\begin{array}{lll}1248.5705 & -1 & 4 \\ 1248.7824 & -3 & 4\end{array}$

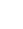




\begin{tabular}{|c|c|c|c|c|c|c|c|}
\hline 20 & 2 & 19 & 21 & 3 & 19 & 1249.0580 & -5 \\
\hline 16 & 3 & 14 & 17 & 4 & 14 & 1249.0962 & -6 \\
\hline 16 & 3 & 13 & 17 & 4 & 13 & 1249.1192 & -4 \\
\hline 22 & 1 & 22 & 23 & 2 & 22 & 1249.4186 & 0 \\
\hline 24 & 0 & 24 & 25 & 1 & 24 & 1249.4968 & 0 \\
\hline 7 & 5 & 2 & 8 & 6 & 2 & 1249.5047 & -2 \\
\hline 24 & 1 & 23 & 25 & 2 & 23 & 1250.1286 & -20 \\
\hline 11 & 4 & 7 & 12 & 5 & 7 & 1250.1286 & 3 \\
\hline 20 & 2 & 18 & 21 & 3 & 18 & 1250.2255 & -4 \\
\hline 15 & 3 & 13 & 16 & 4 & 13 & 1250.6538 & -4 \\
\hline 15 & 3 & 12 & 16 & 4 & 12 & 1250.6698 & -2 \\
\hline 19 & 2 & 18 & 20 & 3 & 18 & 1250.6906 & -5 \\
\hline 6 & 5 & 1 & 7 & 6 & 1 & 1251.0656 & 0 \\
\hline 21 & 1 & 21 & 22 & 2 & 21 & 1251.2695 & 3 \\
\hline 23 & 0 & 23 & 24 & 1 & 23 & 1251.5962 & 0 \\
\hline 19 & 2 & 17 & 20 & 3 & 17 & 1251.6760 & -6 \\
\hline 10 & 4 & 6 & 11 & 5 & 6 & 1251.6870 & 1 \\
\hline 23 & 1 & 22 & 24 & 2 & 22 & 1251.8592 & -8 \\
\hline 14 & 3 & 12 & 15 & 4 & 12 & 1252.2117 & -3 \\
\hline 14 & 3 & 11 & 15 & 4 & 11 & 1252.2224 & -2 \\
\hline 18 & 2 & 17 & 19 & 3 & 17 & 1252.3147 & -6 \\
\hline 5 & 5 & 0 & 6 & 6 & 0 & 1252.6271 & -3 \\
\hline 20 & 1 & 20 & 21 & 2 & 20 & 1253.1098 & -1 \\
\hline 18 & 2 & 16 & 19 & 3 & 16 & 1253.1357 & -4 \\
\hline 9 & 4 & 5 & 10 & 5 & 5 & 1253.2463 & -1 \\
\hline 22 & 1 & 21 & 23 & 2 & 21 & 1253.5615 & -8 \\
\hline 22 & 0 & 22 & 23 & 1 & 22 & 1253.6794 & -5 \\
\hline 13 & 3 & 11 & 14 & 4 & 11 & 1253.7702 & -1 \\
\hline 13 & 3 & 10 & 14 & 4 & 10 & 1253.7771 & -1 \\
\hline 17 & 2 & 16 & 18 & 3 & 16 & 1253.9312 & -4 \\
\hline 17 & 2 & 15 & 18 & 3 & 15 & 1254.6048 & -6 \\
\hline 8 & 4 & 4 & 9 & 5 & 4 & 1254.8067 & -1 \\
\hline 19 & 1 & 19 & 20 & 2 & 19 & 1254.9402 & 1 \\
\hline 7 & 6 & 1 & 7 & 7 & 1 & 1255.0774 & -6 \\
\hline 8 & 6 & 2 & 8 & 7 & 2 & 1255.0854 & -1 \\
\hline 9 & 6 & 3 & 9 & 7 & 3 & 1255.0936 & -4 \\
\hline 10 & 6 & 4 & 10 & 7 & 4 & 1255.1030 & -3 \\
\hline 11 & 6 & 5 & 11 & 7 & 5 & 1255.1130 & -5 \\
\hline 12 & 6 & 6 & 12 & 7 & 6 & 1255.1242 & -3 \\
\hline 13 & 6 & 7 & 13 & 7 & 7 & 1255.1363 & 1 \\
\hline 14 & 6 & 8 & 14 & 7 & 8 & 1255.1487 & -1 \\
\hline 15 & 6 & 9 & 15 & 7 & 9 & 1255.1621 & 1 \\
\hline 16 & 6 & 10 & 16 & 7 & 10 & 1255.1761 & 1 \\
\hline 17 & 6 & 11 & 17 & 7 & 11 & 1255.1908 & 2 \\
\hline 18 & 6 & 12 & 18 & 7 & 12 & 1255.2061 & 3 \\
\hline 19 & 6 & 13 & 19 & 7 & 13 & 1255.2220 & 4 \\
\hline 21 & 1 & 20 & 22 & 2 & 20 & 1255.2378 & -1 \\
\hline 20 & 6 & 14 & 20 & 7 & 14 & 1255.2378 & -1 \\
\hline 21 & 6 & 15 & 21 & 7 & 15 & 1255.2546 & -1 \\
\hline 22 & 6 & 16 & 22 & 7 & 16 & 1255.2721 & 4 \\
\hline 23 & 6 & 17 & 23 & 7 & 17 & 1255.2892 & 4 \\
\hline 24 & 6 & 18 & 24 & 7 & 18 & 1255.3053 & 7 \\
\hline 25 & 6 & 20 & 25 & 7 & 18 & 1255.3168 & -3 \\
\hline 12 & 3 & 10 & 13 & 4 & 10 & 1255.3293 & 1 \\
\hline 12 & 3 & 9 & 13 & 4 & 9 & 1255.3333 & -3 \\
\hline 25 & 6 & 19 & 25 & 7 & 19 & 1255.3447 & 2 \\
\hline 26 & 6 & 20 & 26 & 7 & 20 & 1255.3510 & 9 \\
\hline 26 & 6 & 21 & 26 & 7 & 19 & 1255.3574 & 17 \\
\hline 27 & 6 & 21 & 27 & 7 & 21 & 1255.3689 & 12 \\
\hline 28 & 6 & 22 & 28 & 7 & 22 & 1255.3869 & 5 \\
\hline 29 & 6 & 23 & 29 & 7 & 23 & 1255.4059 & 3 \\
\hline 30 & 6 & 24 & 30 & 7 & 24 & 1255.4240 & -10 \\
\hline 31 & 6 & 25 & 31 & 7 & 25 & 1255.4443 & -1 \\
\hline 16 & 2 & 15 & 17 & 3 & 15 & 1255.5399 & -7 \\
\hline 21 & 0 & 21 & 22 & 1 & 21 & 1255.7454 & -1 \\
\hline 16 & 2 & 14 & 17 & 3 & 14 & 1256.0846 & -4 \\
\hline 7 & 4 & 3 & 8 & 5 & 3 & 1256.3681 & 0 \\
\hline 18 & 1 & 18 & 19 & 2 & 18 & 1256.7595 & 1 \\
\hline 11 & 3 & 9 & 12 & 4 & 9 & 1256.8887 & -1 \\
\hline 20 & 1 & 19 & 21 & 2 & 19 & 1256.8887 & 10 \\
\hline 15 & 2 & 14 & 16 & 3 & 14 & 1257.1425 & -4 \\
\hline 15 & 2 & 13 & 16 & 3 & 13 & 1257.5748 & -3 \\
\hline 20 & 0 & 20 & 21 & 1 & 20 & 1257.7908 & -1 \\
\hline 6 & 4 & 2 & 7 & 5 & 2 & 1257.9303 & 1 \\
\hline 10 & 3 & 7 & 11 & 4 & 7 & 1258.4499 & -7 \\
\hline 19 & 1 & 18 & 20 & 2 & 18 & 1258.5117 & -9 \\
\hline 17 & 1 & 17 & 18 & 2 & 17 & 1258.5673 & -1 \\
\hline
\end{tabular}

\begin{tabular}{|c|c|c|c|c|c|c|c|c|}
\hline 14 & 2 & 13 & 15 & 3 & 13 & 1258.7388 & -2 & 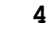 \\
\hline 14 & 2 & 12 & 15 & 3 & 12 & 1259.0753 & -4 & 4 \\
\hline 5 & 4 & 1 & 6 & 5 & 1 & 1259.4932 & 1 & 4 \\
\hline 19 & 0 & 19 & 20 & 1 & 19 & 1259.8141 & -1 & \\
\hline 9 & 3 & 6 & 10 & 4 & 6 & 1260.0105 & -4 & \\
\hline 18 & 1 & 17 & 19 & 2 & 17 & 1260.1128 & -8 & \\
\hline 29 & 2 & 27 & 30 & 1 & 29 & 1260.1256 & 24 & \\
\hline 13 & 2 & 12 & 14 & 3 & 12 & 1260.3293 & -2 & \\
\hline 16 & 1 & 16 & 17 & 2 & 16 & 1260.3638 & 1 & \\
\hline 13 & 2 & 11 & 14 & 3 & 11 & 1260.5862 & 0 & \\
\hline 4 & 4 & 0 & 5 & 5 & 0 & 1261.0570 & 2 & \\
\hline 28 & 2 & 26 & 29 & 1 & 28 & 1261.3713 & 2 & \\
\hline 8 & 3 & 5 & 9 & 4 & 5 & 1261.5721 & -1 & \\
\hline 17 & 1 & 16 & 18 & 2 & 16 & 1261.6914 & -8 & \\
\hline 18 & 0 & 18 & 19 & 1 & 18 & 1261.8140 & 0 & \\
\hline 12 & 2 & 11 & 13 & 3 & 11 & 1261.9152 & 0 & \\
\hline 6 & 5 & 1 & 6 & 6 & 1 & 1262.0334 & 0 & \\
\hline 7 & 5 & 2 & 7 & 6 & 2 & 1262.0387 & -1 & \\
\hline 8 & 5 & 3 & 8 & 6 & 3 & 1262.0452 & 2 & \\
\hline 9 & 5 & 4 & 9 & 6 & 4 & 1262.0520 & 1 & \\
\hline 10 & 5 & 5 & 10 & 6 & 5 & 1262.0595 & -1 & \\
\hline 11 & 5 & 6 & 11 & 6 & 6 & 1262.0678 & -1 & \\
\hline 12 & 5 & 7 & 12 & 6 & 7 & 1262.0769 & 0 & \\
\hline 13 & 5 & 8 & 13 & 6 & 8 & 1262.0867 & 1 & \\
\hline 14 & 5 & 9 & 14 & 6 & 9 & 1262.0968 & -2 & \\
\hline 15 & 5 & 10 & 15 & 6 & 10 & 1262.1069 & -11 & 10 \\
\hline 12 & 2 & 10 & 13 & 3 & 10 & 1262.1069 & 8 & 10 \\
\hline 16 & 5 & 11 & 16 & 6 & 11 & 1262.1193 & -3 & \\
\hline 17 & 5 & 12 & 17 & 6 & 12 & 1262.1315 & -4 & \\
\hline 18 & 5 & 13 & 18 & 6 & 13 & 1262.1450 & -1 & \\
\hline 19 & 5 & 14 & 19 & 6 & 14 & 1262.1579 & -14 & \\
\hline 20 & 5 & 16 & 20 & 6 & 14 & 1262.1704 & -1 & \\
\hline 20 & 5 & 15 & 20 & 6 & 15 & 1262.1758 & -5 & \\
\hline 21 & 5 & 17 & 21 & 6 & 15 & 1262.1848 & 4 & 10 \\
\hline 22 & 5 & 17 & 22 & 6 & 17 & 1262.1848 & 9 & 10 \\
\hline 22 & 5 & 18 & 22 & 6 & 16 & 1262.1988 & 0 & 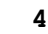 \\
\hline 23 & 5 & 18 & 23 & 6 & 18 & 1262.2051 & 6 & \\
\hline 23 & 5 & 19 & 23 & 6 & 17 & 1262.2122 & -13 & 10 \\
\hline 21 & 5 & 16 & 21 & 6 & 16 & 1262.2122 & 5 & 10 \\
\hline 24 & 5 & 19 & 24 & 6 & 19 & 1262.2213 & 1 & \\
\hline 24 & 5 & 20 & 24 & 6 & 18 & 1262.2289 & 4 & \\
\hline 25 & 5 & 20 & 25 & 6 & 20 & 1262.2369 & -2 & \\
\hline 25 & 5 & 21 & 25 & 6 & 19 & 1262.2440 & 1 & \\
\hline 26 & 5 & 21 & 26 & 6 & 21 & 1262.2524 & -4 & \\
\hline 26 & 5 & 22 & 26 & 6 & 20 & 1262.2593 & -1 & \\
\hline 27 & 5 & 22 & 27 & 6 & 22 & 1262.2675 & -10 & \\
\hline 27 & 5 & 23 & 27 & 6 & 21 & 1262.2749 & -3 & \\
\hline 28 & 5 & 23 & 28 & 6 & 23 & 1262.2818 & -25 & \\
\hline 28 & 5 & 24 & 28 & 6 & 22 & 1262.2921 & 10 & \\
\hline 29 & 5 & 24 & 29 & 6 & 24 & 1262.2977 & -24 & \\
\hline 27 & 2 & 25 & 28 & 1 & 27 & 1262.6674 & 7 & \\
\hline 7 & 3 & 4 & 8 & 4 & 4 & 1263.1346 & 1 & \\
\hline 16 & 1 & 15 & 17 & 2 & 15 & 1263.2493 & -7 & \\
\hline 11 & 2 & 10 & 12 & 3 & 10 & 1263.4969 & 3 & \\
\hline 11 & 2 & 9 & 12 & 3 & 9 & 1263.6347 & 0 & \\
\hline 17 & 0 & 17 & 18 & 1 & 17 & 1263.7890 & -1 & \\
\hline 14 & 1 & 14 & 15 & 2 & 14 & 1263.9202 & 2 & \\
\hline 26 & 2 & 24 & 27 & 1 & 26 & 1264.0091 & 5 & \\
\hline 6 & 3 & 3 & 7 & 4 & 3 & 1264.6979 & 3 & \\
\hline 15 & 1 & 14 & 16 & 2 & 14 & 1264.7884 & -6 & \\
\hline 28 & 3 & 25 & 29 & 2 & 27 & 1264.9358 & 18 & \\
\hline 10 & 2 & 9 & 11 & 3 & 9 & 1265.0744 & 1 & \\
\hline 10 & 2 & 8 & 11 & 3 & 8 & 1265.1710 & 0 & \\
\hline 25 & 2 & 23 & 26 & 1 & 25 & 1265.3943 & -8 & \\
\hline 13 & 1 & 13 & 14 & 2 & 13 & 1265.6797 & 2 & \\
\hline 16 & 0 & 16 & 17 & 1 & 16 & 1265.7390 & 0 & \\
\hline 5 & 3 & 2 & 6 & 4 & 2 & 1266.2617 & 2 & \\
\hline 14 & 1 & 13 & 15 & 2 & 13 & 1266.3106 & -6 & \\
\hline 9 & 2 & 8 & 10 & 3 & 8 & 1266.6491 & 2 & \\
\hline 9 & 2 & 7 & 10 & 3 & 7 & 1266.7142 & 2 & \\
\hline 27 & 3 & 24 & 28 & 2 & 26 & 1266.7889 & 14 & \\
\hline 24 & 2 & 22 & 25 & 1 & 24 & 1266.8237 & -2 & \\
\hline 12 & 1 & 12 & 13 & 2 & 12 & 1267.4263 & 2 & \\
\hline 15 & 0 & 15 & 16 & 1 & 15 & 1267.6630 & -2 & \\
\hline 13 & 1 & 12 & 14 & 2 & 12 & 1267.8188 & -4 & \\
\hline 4 & 3 & 1 & 5 & 4 & 1 & 1267.8264 & 4 & \\
\hline 8 & 2 & 7 & 9 & 3 & 7 & 1268.2215 & 5 & \\
\hline
\end{tabular}




\begin{tabular}{|c|c|c|c|c|c|c|c|}
\hline 32 & 3 & 30 & 32 & 4 & 28 & 1275.3881 & -7 \\
\hline 31 & 3 & 29 & 31 & 4 & 27 & 1275.4732 & -1 \\
\hline 13 & 6 & 7 & 12 & 7 & 5 & 1275.4922 & 0 \\
\hline 30 & 3 & 28 & 30 & 4 & 26 & 1275.5424 & -4 \\
\hline 29 & 3 & 27 & 29 & 4 & 25 & 1275.5981 & -9 \\
\hline 28 & 3 & 26 & 28 & 4 & 24 & 1275.6442 & 4 \\
\hline 7 & 3 & 4 & 7 & 4 & 4 & 1275.6728 & -3 \\
\hline 8 & 3 & 5 & 8 & 4 & 5 & 1275.6773 & -4 \\
\hline 9 & 3 & 6 & 9 & 4 & 6 & 1275.6828 & -2 \\
\hline 10 & 3 & 7 & 10 & 4 & 7 & 1275.6887 & -5 \\
\hline 11 & 3 & 8 & 11 & 4 & 8 & 1275.6951 & -13 \\
\hline 12 & 3 & 10 & 12 & 4 & 8 & 1275.6989 & -11 \\
\hline 12 & 3 & 9 & 12 & 4 & 9 & 1275.7057 & 11 \\
\hline 13 & 3 & 10 & 13 & 4 & 10 & 1275.7139 & -3 \\
\hline 15 & 3 & 13 & 15 & 4 & 11 & 1275.7215 & 5 \\
\hline 14 & 3 & 11 & 14 & 4 & 11 & 1275.7262 & 9 \\
\hline 15 & 3 & 12 & 15 & 4 & 12 & 1275.7380 & -1 \\
\hline 19 & 3 & 17 & 19 & 4 & 15 & 1275.7452 & -6 \\
\hline 17 & 3 & 14 & 17 & 4 & 14 & 1275.7705 & -2 \\
\hline 18 & 3 & 15 & 18 & 4 & 15 & 1275.7912 & -1 \\
\hline 19 & 3 & 16 & 19 & 4 & 16 & 1275.8152 & -2 \\
\hline 20 & 3 & 17 & 20 & 4 & 17 & 1275.8438 & 0 \\
\hline 21 & 3 & 18 & 21 & 4 & 18 & 1275.8770 & 0 \\
\hline 18 & 1 & 17 & 19 & 0 & 19 & 1275.9083 & -8 \\
\hline 22 & 3 & 19 & 22 & 4 & 19 & 1275.9158 & -2 \\
\hline 7 & 1 & 7 & 8 & 2 & 7 & 1275.9616 & 5 \\
\hline 24 & 3 & 21 & 24 & 4 & 21 & 1276.0154 & 4 \\
\hline 22 & 3 & 19 & 23 & 2 & 21 & 1276.0436 & 3 \\
\hline 3 & 2 & 2 & 4 & 3 & 2 & 1276.0622 & 10 \\
\hline 25 & 3 & 22 & 25 & 4 & 22 & 1276.0780 & 9 \\
\hline 18 & 2 & 16 & 19 & 1 & 18 & 1276.1446 & -5 \\
\hline 26 & 3 & 23 & 26 & 4 & 23 & 1276.1508 & 16 \\
\hline 27 & 3 & 24 & 27 & 4 & 24 & 1276.2343 & 19 \\
\hline 28 & 3 & 25 & 28 & 4 & 25 & 1276.3298 & 15 \\
\hline 18 & 7 & 11 & 17 & 8 & 9 & 1276.3426 & 5 \\
\hline 23 & 2 & 22 & 24 & 1 & 24 & 1276.3866 & -2 \\
\hline 29 & 3 & 26 & 29 & 4 & 26 & 1276.4399 & 20 \\
\hline 30 & 3 & 27 & 30 & 4 & 27 & 1276.5652 & 25 \\
\hline 31 & 3 & 28 & 31 & 4 & 28 & 1276.7056 & 16 \\
\hline 7 & 1 & 6 & 8 & 2 & 6 & 1276.7227 & 2 \\
\hline 10 & 0 & 10 & 11 & 1 & 10 & 1276.9074 & 1 \\
\hline 17 & 1 & 16 & 18 & 0 & 18 & 1276.9707 & -5 \\
\hline 26 & 4 & 22 & 27 & 3 & 24 & 1276.9981 & 7 \\
\hline 14 & 6 & 8 & 13 & 7 & 6 & 1277.0691 & 6 \\
\hline 22 & 2 & 21 & 23 & 1 & 23 & 1277.4508 & -9 \\
\hline 2 & 2 & 1 & 3 & 3 & 1 & 1277.6284 & 5 \\
\hline 6 & 1 & 6 & 7 & 2 & 6 & 1277.6284 & 10 \\
\hline 10 & 5 & 5 & 9 & 6 & 3 & 1277.7242 & -10 \\
\hline 17 & 2 & 15 & 18 & 1 & 17 & 1277.7959 & -5 \\
\hline 21 & 3 & 18 & 22 & 2 & 20 & 1277.8690 & 1 \\
\hline 19 & 7 & 12 & 18 & 8 & 10 & 1277.9232 & 12 \\
\hline 16 & 1 & 15 & 17 & 0 & 17 & 1278.0737 & -7 \\
\hline 6 & 1 & 5 & 7 & 2 & 5 & 1278.2083 & 2 \\
\hline 21 & 2 & 20 & 22 & 1 & 22 & 1278.5382 & -8 \\
\hline 15 & 6 & 9 & 14 & 7 & 7 & 1278.6451 & 1 \\
\hline 9 & 0 & 9 & 10 & 1 & 9 & 1278.6850 & 1 \\
\hline 25 & 4 & 21 & 26 & 3 & 23 & 1278.7133 & 1 \\
\hline 22 & 3 & 20 & 23 & 2 & 22 & 1279.0345 & -7 \\
\hline 15 & 1 & 14 & 16 & 0 & 16 & 1279.2179 & -5 \\
\hline 5 & 1 & 5 & 6 & 2 & 5 & 1279.2804 & 4 \\
\hline 11 & 5 & 6 & 10 & 6 & 4 & 1279.2983 & -6 \\
\hline 25 & 4 & 22 & 26 & 3 & 24 & 1279.3953 & 8 \\
\hline 16 & 2 & 14 & 17 & 1 & 16 & 1279.4666 & -4 \\
\hline 20 & 7 & 13 & 19 & 8 & 11 & 1279.5030 & 10 \\
\hline 20 & 2 & 19 & 21 & 1 & 21 & 1279.6479 & -11 \\
\hline 20 & 3 & 17 & 21 & 2 & 19 & 1279.6788 & -7 \\
\hline 5 & 1 & 4 & 6 & 2 & 4 & 1279.7013 & 5 \\
\hline 7 & 4 & 3 & 6 & 5 & 1 & 1279.8747 & 6 \\
\hline 28 & 2 & 27 & 28 & 3 & 25 & 1279.9486 & -10 \\
\hline 16 & 6 & 10 & 15 & 7 & 8 & 1280.2216 & -2 \\
\hline 27 & 2 & 26 & 27 & 3 & 24 & 1280.2996 & -4 \\
\hline 14 & 1 & 13 & 15 & 0 & 15 & 1280.4018 & -5 \\
\hline 8 & 0 & 8 & 9 & 1 & 8 & 1280.4410 & 3 \\
\hline 21 & 3 & 19 & 22 & 2 & 21 & 1280.4625 & -2 \\
\hline 26 & 2 & 25 & 26 & 3 & 23 & 1280.6104 & -2 \\
\hline 19 & 2 & 18 & 20 & 1 & 20 & 1280.7816 & -2 \\
\hline 12 & 5 & 7 & 11 & 6 & 5 & 1280.8734 & 4 \\
\hline
\end{tabular}




\begin{tabular}{|c|c|c|c|c|c|c|c|c|}
\hline 25 & 2 & 24 & 25 & 3 & 22 & 1280.8831 & -10 & 4 \\
\hline 4 & 1 & 4 & 5 & 2 & 4 & 1280.9191 & 5 & 4 \\
\hline 24 & 4 & 21 & 25 & 3 & 23 & 1280.9591 & 8 & 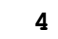 \\
\hline 24 & 1 & 24 & 24 & 2 & 22 & 1281.0361 & 4 & 4 \\
\hline 21 & 7 & 14 & 20 & 8 & 12 & 1281.0828 & 9 & \\
\hline 24 & 2 & 23 & 24 & 3 & 21 & 1281.1226 & -10 & 4 \\
\hline 15 & 2 & 13 & 16 & 1 & 15 & 1281.1536 & -2 & \\
\hline 4 & 1 & 3 & 5 & 2 & 3 & 1281.2031 & 4 & \\
\hline 23 & 2 & 22 & 23 & 3 & 20 & 1281.3309 & -8 & \\
\hline 8 & 4 & 4 & 7 & 5 & 2 & 1281.4464 & 6 & \\
\hline 19 & 3 & 16 & 20 & 2 & 18 & 1281.4728 & -5 & 4 \\
\hline 22 & 2 & 21 & 22 & 3 & 19 & 1281.5108 & -6 & \\
\hline 13 & 1 & 12 & 14 & 0 & 14 & 1281.6245 & -4 & \\
\hline 21 & 2 & 20 & 21 & 3 & 18 & 1281.6647 & -8 & \\
\hline 20 & 2 & 19 & 20 & 3 & 17 & 1281.7965 & 0 & \\
\hline 23 & 1 & 23 & 23 & 2 & 21 & 1281.8352 & 1 & \\
\hline 19 & 2 & 18 & 19 & 3 & 16 & 1281.9061 & -10 & 10 \\
\hline 20 & 3 & 18 & 21 & 2 & 20 & 1281.9061 & 6 & 10 \\
\hline 18 & 2 & 17 & 19 & 1 & 19 & 1281.9373 & -1 & \\
\hline 18 & 2 & 17 & 18 & 3 & 15 & 1281.9989 & -6 & 4 \\
\hline 17 & 2 & 16 & 17 & 3 & 14 & 1282.0755 & -4 & \\
\hline 23 & 4 & 19 & 24 & 3 & 21 & 1282.0877 & 1 & 4 \\
\hline 16 & 2 & 15 & 16 & 3 & 13 & 1282.1382 & -2 & \\
\hline 7 & 0 & 7 & 8 & 1 & 7 & 1282.1757 & 3 & 4 \\
\hline 15 & 2 & 14 & 15 & 3 & 12 & 1282.1886 & -3 & \\
\hline 14 & 2 & 13 & 14 & 3 & 11 & 1282.2288 & -3 & \\
\hline 13 & 2 & 12 & 13 & 3 & 10 & 1282.2604 & 0 & 4 \\
\hline 12 & 2 & 11 & 12 & 3 & 9 & 1282.2842 & -1 & \\
\hline 11 & 2 & 10 & 11 & 3 & 8 & 1282.3021 & 0 & 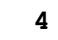 \\
\hline 10 & 2 & 9 & 10 & 3 & 7 & 1282.3149 & 1 & 4 \\
\hline 9 & 2 & 8 & 9 & 3 & 6 & 1282.3237 & 2 & \\
\hline 5 & 2 & 3 & 5 & 3 & 3 & 1282.3410 & 2 & 4 \\
\hline 6 & 2 & 4 & 6 & 3 & 4 & 1282.3484 & 4 & 4 \\
\hline 7 & 2 & 5 & 7 & 3 & 5 & 1282.3586 & 2 & 4 \\
\hline 8 & 2 & 6 & 8 & 3 & 6 & 1282.3733 & 3 & 4 \\
\hline 9 & 2 & 7 & 9 & 3 & 7 & 1282.3928 & 0 & 4 \\
\hline 10 & 2 & 8 & 10 & 3 & 8 & 1282.4191 & 0 & \\
\hline 13 & 5 & 8 & 12 & 6 & 6 & 1282.4469 & -4 & 4 \\
\hline 11 & 2 & 9 & 11 & 3 & 9 & 1282.4531 & 0 & \\
\hline 12 & 2 & 10 & 12 & 3 & 10 & 1282.4960 & -1 & 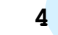 \\
\hline 23 & 4 & 20 & 24 & 3 & 22 & 1282.5247 & -2 & \\
\hline 3 & 1 & 3 & 4 & 2 & 3 & 1282.5437 & 4 & 4 \\
\hline 13 & 2 & 11 & 13 & 3 & 11 & 1282.5496 & 0 & \\
\hline 22 & 1 & 22 & 22 & 2 & 20 & 1282.5870 & 8 & \\
\hline 14 & 2 & 12 & 14 & 3 & 12 & 1282.6148 & -2 & \\
\hline 22 & 7 & 15 & 21 & 8 & 13 & 1282.6626 & 9 & \\
\hline 15 & 2 & 13 & 15 & 3 & 13 & 1282.6933 & -4 & 4 \\
\hline 3 & 1 & 2 & 4 & 2 & 2 & 1282.7162 & 6 & \\
\hline 16 & 2 & 14 & 16 & 3 & 14 & 1282.7865 & -5 & \\
\hline 14 & 2 & 12 & 15 & 1 & 14 & 1282.8531 & -4 & \\
\hline 12 & 1 & 11 & 13 & 0 & 13 & 1282.8842 & -4 & \\
\hline 17 & 2 & 15 & 17 & 3 & 15 & 1282.8958 & -4 & \\
\hline 18 & 2 & 16 & 18 & 3 & 16 & 1283.0214 & -9 & \\
\hline 17 & 2 & 16 & 18 & 1 & 18 & 1283.1152 & -6 & \\
\hline 19 & 2 & 17 & 19 & 3 & 17 & 1283.1657 & -5 & \\
\hline 18 & 3 & 15 & 19 & 2 & 17 & 1283.2481 & -5 & \\
\hline 21 & 1 & 21 & 21 & 2 & 19 & 1283.2885 & 3 & \\
\hline 20 & 2 & 18 & 20 & 3 & 18 & 1283.3284 & -3 & \\
\hline 19 & 3 & 17 & 20 & 2 & 19 & 1283.3622 & -6 & \\
\hline 18 & 6 & 12 & 17 & 7 & 10 & 1283.3758 & 2 & \\
\hline 21 & 2 & 19 & 21 & 3 & 19 & 1283.5100 & -3 & \\
\hline 22 & 2 & 20 & 22 & 3 & 20 & 1283.7111 & -2 & \\
\hline 22 & 4 & 18 & 23 & 3 & 20 & 1283.7502 & 7 & \\
\hline 6 & 0 & 6 & 7 & 1 & 6 & 1283.8901 & 3 & \\
\hline 23 & 2 & 21 & 23 & 3 & 21 & 1283.9319 & 1 & \\
\hline 20 & 1 & 20 & 20 & 2 & 18 & 1283.9416 & 6 & \\
\hline 14 & 5 & 9 & 13 & 6 & 7 & 1284.0218 & -1 & \\
\hline 22 & 4 & 19 & 23 & 3 & 21 & 1284.0939 & 2 & \\
\hline 2 & 1 & 2 & 3 & 2 & 2 & 1284.1543 & 5 & \\
\hline 24 & 2 & 22 & 24 & 3 & 22 & 1284.1718 & 1 & 4 \\
\hline 11 & 1 & 10 & 12 & 0 & 12 & 1284.1793 & -3 & \\
\hline 23 & 7 & 16 & 22 & 8 & 14 & 1284.2414 & 1 & 10 \\
\hline 2 & 1 & 1 & 3 & 2 & 1 & 1284.2414 & 6 & 10 \\
\hline 16 & 2 & 15 & 17 & 1 & 17 & 1284.3168 & -1 & \\
\hline 25 & 2 & 23 & 25 & 3 & 23 & 1284.4313 & 5 & \\
\hline 19 & 1 & 19 & 19 & 2 & 17 & 1284.5451 & 6 & \\
\hline 13 & 2 & 11 & 14 & 1 & 13 & 1284.5626 & 0 & \\
\hline
\end{tabular}

\begin{tabular}{|c|c|c|c|c|c|c|c|c|}
\hline 10 & 4 & 6 & 9 & 5 & 4 & 1284.5904 & 1 & 4 \\
\hline 26 & 2 & 24 & 26 & 3 & 24 & 1284.7092 & 5 & 4 \\
\hline 18 & 3 & 16 & 19 & 2 & 18 & 1284.8330 & -4 & 4 \\
\hline 19 & 6 & 13 & 18 & 7 & 11 & 1284.9526 & 0 & 4 \\
\hline 27 & 2 & 25 & 27 & 3 & 25 & 1285.0048 & -2 & 10 \\
\hline 17 & 3 & 14 & 18 & 2 & 16 & 1285.0048 & 5 & 10 \\
\hline 6 & 3 & 3 & 5 & 4 & 1 & 1285.0736 & -1 & 4 \\
\hline 18 & 1 & 18 & 18 & 2 & 16 & 1285.0991 & -1 & 4 \\
\hline 28 & 2 & 26 & 28 & 3 & 26 & 1285.3208 & 18 & 4 \\
\hline 21 & 4 & 17 & 22 & 3 & 19 & 1285.3954 & -12 & 4 \\
\hline 10 & 1 & 9 & 11 & 0 & 11 & 1285.5077 & -1 & 4 \\
\hline 15 & 2 & 14 & 16 & 1 & 16 & 1285.5406 & -1 & 4 \\
\hline 5 & 0 & 5 & 6 & 1 & 5 & 1285.5851 & 5 & 4 \\
\hline 15 & 5 & 10 & 14 & 6 & 8 & 1285.5962 & -6 & 4 \\
\hline 17 & 1 & 17 & 17 & 2 & 15 & 1285.6057 & -2 & 4 \\
\hline 29 & 2 & 27 & 29 & 3 & 27 & 1285.6515 & 15 & 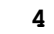 \\
\hline 21 & 4 & 18 & 22 & 3 & 20 & 1285.6643 & 1 & 4 \\
\hline 1 & 1 & 1 & 2 & 2 & 1 & 1285.7510 & 7 & 4 \\
\hline 1 & 1 & 0 & 2 & 2 & 0 & 1285.7800 & 6 & 4 \\
\hline 24 & 7 & 17 & 23 & 8 & 15 & 1285.8205 & 0 & 4 \\
\hline 30 & 2 & 28 & 30 & 3 & 28 & 1285.9994 & 21 & 4 \\
\hline 16 & 1 & 16 & 16 & 2 & 14 & 1286.0659 & 0 & 4 \\
\hline 11 & 4 & 7 & 10 & 5 & 5 & 1286.1630 & -1 & 4 \\
\hline 12 & 2 & 10 & 13 & 1 & 12 & 1286.2780 & 0 & 4 \\
\hline 17 & 3 & 15 & 18 & 2 & 17 & 1286.3157 & -8 & 4 \\
\hline 31 & 2 & 29 & 31 & 3 & 29 & 1286.3631 & 31 & 4 \\
\hline 15 & 1 & 15 & 15 & 2 & 13 & 1286.4809 & 1 & 4 \\
\hline 20 & 6 & 14 & 19 & 7 & 12 & 1286.5300 & 6 & 4 \\
\hline 7 & 3 & 4 & 6 & 4 & 2 & 1286.6442 & -5 & 4 \\
\hline 16 & 3 & 13 & 17 & 2 & 15 & 1286.7397 & -2 & 4 \\
\hline 32 & 2 & 30 & 32 & 3 & 30 & 1286.7397 & 24 & 4 \\
\hline 14 & 2 & 13 & 15 & 1 & 15 & 1286.7869 & -1 & 4 \\
\hline 14 & 1 & 14 & 14 & 2 & 12 & 1286.8526 & 0 & 4 \\
\hline 9 & 1 & 8 & 10 & 0 & 10 & 1286.8669 & -1 & 4 \\
\hline 20 & 4 & 16 & 21 & 3 & 18 & 1287.0307 & 1 & 4 \\
\hline 16 & 5 & 11 & 15 & 6 & 9 & 1287.1715 & -3 & 4 \\
\hline 13 & 1 & 13 & 13 & 2 & 11 & 1287.1837 & 2 & 4 \\
\hline 20 & 4 & 17 & 21 & 3 & 19 & 1287.2360 & 1 & 4 \\
\hline 4 & 0 & 4 & 5 & 1 & 4 & 1287.2608 & 4 & 4 \\
\hline 25 & 7 & 18 & 24 & 8 & 16 & 1287.4001 & 8 & 4 \\
\hline 12 & 1 & 12 & 12 & 2 & 10 & 1287.4766 & 5 & 4 \\
\hline 11 & 1 & 11 & 11 & 2 & 9 & 1287.7340 & 10 & 4 \\
\hline 16 & 3 & 14 & 17 & 2 & 16 & 1287.8105 & -5 & 4 \\
\hline 10 & 1 & 10 & 10 & 2 & 8 & 1287.9574 & 4 & 4 \\
\hline 11 & 2 & 9 & 12 & 1 & 11 & 1287.9966 & 1 & 4 \\
\hline 13 & 2 & 12 & 14 & 1 & 14 & 1288.0556 & 1 & 4 \\
\hline 21 & 6 & 15 & 20 & 7 & 13 & 1288.1061 & 1 & 4 \\
\hline 9 & 1 & 9 & 9 & 2 & 7 & 1288.1510 & 3 & 4 \\
\hline 8 & 3 & 5 & 7 & 4 & 3 & 1288.2160 & -3 & 4 \\
\hline 8 & 1 & 7 & 9 & 0 & 9 & 1288.2551 & 1 & 4 \\
\hline 8 & 1 & 8 & 8 & 2 & 6 & 1288.3173 & 6 & 4 \\
\hline 7 & 1 & 7 & 7 & 2 & 5 & 1288.4573 & -4 & 10 \\
\hline 23 & 5 & 19 & 24 & 4 & 21 & 1288.4853 & 5 & 4 \\
\hline 6 & 1 & 6 & 6 & 2 & 4 & 1288.5762 & 5 & 4 \\
\hline 19 & 4 & 15 & 20 & 3 & 17 & 1288.6530 & 0 & 4 \\
\hline 5 & 1 & 5 & 5 & 2 & 3 & 1288.6736 & 6 & 4 \\
\hline 17 & 5 & 12 & 16 & 6 & 10 & 1288.7466 & -4 & 4 \\
\hline 4 & 1 & 4 & 4 & 2 & 2 & 1288.7519 & 6 & 4 \\
\hline 3 & 1 & 3 & 3 & 2 & 1 & 1288.8122 & 1 & 4 \\
\hline 2 & 1 & 2 & 2 & 2 & 0 & 1288.8573 & 7 & 4 \\
\hline 3 & 0 & 3 & 4 & 1 & 3 & 1288.9183 & 6 & 4 \\
\hline 2 & 1 & 1 & 2 & 2 & 1 & 1288.9461 & 7 & 4 \\
\hline 26 & 7 & 19 & 25 & 8 & 17 & 1288.9771 & -5 & 4 \\
\hline 3 & 1 & 2 & 3 & 2 & 2 & 1288.9911 & 6 & 4 \\
\hline 4 & 1 & 3 & 4 & 2 & 3 & 1289.0513 & 6 & 4 \\
\hline 5 & 1 & 4 & 5 & 2 & 4 & 1289.1262 & 3 & 4 \\
\hline 6 & 1 & 5 & 6 & 2 & 5 & 1289.2163 & 2 & 4 \\
\hline 13 & 4 & 9 & 12 & 5 & 7 & 1289.3095 & -1 & 4 \\
\hline 15 & 3 & 13 & 16 & 2 & 15 & 1289.3162 & 2 & 4 \\
\hline 7 & 1 & 6 & 7 & 2 & 6 & 1289.3214 & 2 & 4 \\
\hline 12 & 2 & 11 & 13 & 1 & 13 & 1289.3461 & 0 & 4 \\
\hline 8 & 1 & 7 & 8 & 2 & 7 & 1289.4413 & 1 & 4 \\
\hline 9 & 1 & 8 & 9 & 2 & 8 & 1289.5762 & 1 & 4 \\
\hline 7 & 1 & 6 & 8 & 0 & 8 & 1289.6697 & 0 & 4 \\
\hline 22 & 6 & 16 & 21 & 7 & 14 & 1289.6827 & 5 & 4 \\
\hline 10 & 2 & 8 & 11 & 1 & 10 & 1289.7153 & 1 & 4 \\
\hline 10 & 1 & 9 & 10 & 2 & 9 & 1289.7256 & -2 & 4 \\
\hline
\end{tabular}



$\begin{array}{rrrrrrrrr}11 & 3 & 8 & 12 & 2 & 10 & 1295.1253 & -6 & 10\end{array}$ $\begin{array}{rrrrrrrrr}30 & 1 & 29 & 30 & 2 & 29 & 1295.2568 & -1 & 4\end{array}$

17

13$$
2
$$

22 


\begin{tabular}{|c|c|c|c|c|c|c|c|c|}
\hline 7 & 1 & 7 & 6 & 2 & 5 & 1299.4661 & 4 & 4 \\
\hline 16 & 5 & 11 & 17 & 4 & 13 & 1299.5019 & 18 & 10 \\
\hline 26 & 0 & 26 & 26 & 1 & 26 & 1299.5321 & 1 & 4 \\
\hline 11 & 2 & 10 & 10 & 3 & 8 & 1299.5499 & -3 & 4 \\
\hline 11 & 2 & 9 & 10 & 3 & 7 & 1299.6935 & -1 & 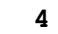 \\
\hline 27 & 0 & 27 & 27 & 1 & 27 & 1299.7335 & 1 & 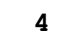 \\
\hline 24 & 5 & 19 & 23 & 6 & 17 & 1299.7606 & 5 & A \\
\hline 24 & 5 & 20 & 23 & 6 & 18 & 1299.7666 & -9 & 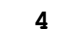 \\
\hline 12 & 4 & 8 & 13 & 3 & 10 & 1299.7978 & -4 & 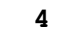 \\
\hline 12 & 4 & 9 & 13 & 3 & 11 & 1299.8112 & 3 & 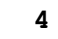 \\
\hline 4 & 2 & 2 & 5 & 1 & 4 & 1299.8884 & 2 & 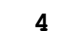 \\
\hline 28 & 0 & 28 & 28 & 1 & 28 & 1299.9344 & 3 & $z$ \\
\hline 8 & 3 & 5 & 9 & 2 & 7 & 1299.9672 & -2 & 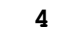 \\
\hline 3 & 0 & 3 & 2 & 1 & 1 & 1300.0183 & 7 & 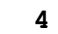 \\
\hline 8 & 3 & 6 & 9 & 2 & 8 & 1300.0669 & 0 & 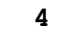 \\
\hline 29 & 0 & 29 & 29 & 1 & 29 & 1300.1348 & 4 & 4 \\
\hline 23 & 7 & 16 & 24 & 6 & 18 & 1300.2147 & 9 & 4 \\
\hline 7 & 1 & 6 & 6 & 2 & 4 & 1300.2697 & 2 & 4 \\
\hline 21 & 1 & 21 & 21 & 0 & 21 & 1300.3084 & -1 & 4 \\
\hline 20 & 1 & 20 & 20 & 0 & 20 & 1300.3160 & -6 & 10 \\
\hline 22 & 1 & 22 & 22 & 0 & 22 & 1300.3160 & 3 & 10 \\
\hline 20 & 4 & 16 & 19 & 5 & 14 & 1300.3271 & -22 & 4 \\
\hline 19 & 1 & 19 & 19 & 0 & 19 & 1300.3390 & -10 & 10 \\
\hline 23 & 1 & 23 & 23 & 0 & 23 & 1300.3390 & 8 & 10 \\
\hline 18 & 1 & 18 & 18 & 0 & 18 & 1300.3772 & -9 & 10 \\
\hline 24 & 1 & 24 & 24 & 0 & 24 & 1300.3772 & 14 & 10 \\
\hline 17 & 1 & 17 & 17 & 0 & 17 & 1300.4291 & -12 & 10 \\
\hline 25 & 1 & 25 & 25 & 0 & 25 & 1300.4291 & 12 & 10 \\
\hline 16 & 1 & 16 & 16 & 0 & 16 & 1300.4950 & -3 & 10 \\
\hline 26 & 1 & 26 & 26 & 0 & 26 & 1300.4950 & 9 & 10 \\
\hline 31 & 0 & 31 & 31 & 1 & 31 & 1300.5364 & 9 & 4 \\
\hline 27 & 1 & 27 & 27 & 0 & 27 & 1300.5724 & -13 & 10 \\
\hline 15 & 1 & 15 & 15 & 0 & 15 & 1300.5724 & 5 & 10 \\
\hline 14 & 1 & 14 & 14 & 0 & 14 & 1300.6585 & 3 & 4 \\
\hline 28 & 1 & 28 & 28 & 0 & 28 & 1300.6665 & 4 & 4 \\
\hline 19 & 6 & 13 & 20 & 5 & 15 & 1300.6788 & 7 & 4 \\
\hline 32 & 0 & 32 & 32 & 1 & 32 & 1300.7381 & 11 & 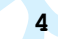 \\
\hline 13 & 1 & 13 & 13 & 0 & 13 & 1300.7526 & 1 & 4 \\
\hline 29 & 1 & 29 & 29 & 0 & 29 & 1300.7708 & 3 & 4 \\
\hline 16 & 3 & 14 & 15 & 4 & 12 & 1300.7958 & -4 & 4 \\
\hline 16 & 3 & 13 & 15 & 4 & 11 & 1300.8193 & -6 & 4 \\
\hline 12 & 1 & 12 & 12 & 0 & 12 & 1300.8526 & 1 & 4 \\
\hline 30 & 1 & 30 & 30 & 0 & 30 & 1300.8867 & 5 & 4 \\
\hline 8 & 1 & 8 & 7 & 2 & 6 & 1300.9159 & 4 & 4 \\
\hline 33 & 0 & 33 & 33 & 1 & 33 & 1300.9406 & 9 & 4 \\
\hline 11 & 1 & 11 & 11 & 0 & 11 & 1300.9562 & 3 & 4 \\
\hline 31 & 1 & 31 & 31 & 0 & 31 & 1301.0134 & 8 & \\
\hline 10 & 1 & 10 & 10 & 0 & 10 & 1301.0608 & 3 & 4 \\
\hline 15 & 5 & 10 & 16 & 4 & 12 & 1301.0691 & 2 & 4 \\
\hline 12 & 2 & 11 & 11 & 3 & 9 & 1301.1029 & 2 & 4 \\
\hline 34 & 0 & 34 & 34 & 1 & 34 & 1301.1455 & 17 & 4 \\
\hline 32 & 1 & 32 & 32 & 0 & 32 & 1301.1500 & 10 & 4 \\
\hline 9 & 1 & 9 & 9 & 0 & 9 & 1301.1644 & 4 & 4 \\
\hline 35 & 2 & 34 & 35 & 1 & 34 & 1301.2071 & -15 & 4 \\
\hline 8 & 1 & 8 & 8 & 0 & 8 & 1301.2643 & 3 & \\
\hline 33 & 1 & 33 & 33 & 0 & 33 & 1301.2963 & 16 & 4 \\
\hline 12 & 2 & 10 & 11 & 3 & 8 & 1301.3015 & -1 & $A$ \\
\hline 34 & 2 & 33 & 34 & 1 & 33 & 1301.3235 & -24 & 4 \\
\hline 25 & 5 & 20 & 24 & 6 & 18 & 1301.3343 & 4 & 4 \\
\hline 25 & 5 & 21 & 24 & 6 & 19 & 1301.3411 & 4 & \\
\hline 35 & 0 & 35 & 35 & 1 & 35 & 1301.3514 & 18 & \\
\hline 7 & 1 & 7 & 7 & 0 & 7 & 1301.3589 & 4 & 4 \\
\hline 11 & 4 & 7 & 12 & 3 & 9 & 1301.3730 & 6 & 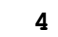 \\
\hline 11 & 4 & 8 & 12 & 3 & 10 & 1301.3805 & 1 & \\
\hline 6 & 1 & 6 & 6 & 0 & 6 & 1301.4459 & 4 & 4 \\
\hline 33 & 2 & 32 & 33 & 1 & 32 & 1301.4636 & -12 & 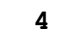 \\
\hline 5 & 1 & 5 & 5 & 0 & 5 & 1301.5238 & 4 & \\
\hline 4 & 0 & 4 & 3 & 1 & 2 & 1301.5310 & 8 & 4 \\
\hline 3 & 2 & 1 & 4 & 1 & 3 & 1301.5428 & 11 & 4 \\
\hline 7 & 3 & 4 & 8 & 2 & 6 & 1301.5599 & 0 & 10 \\
\hline 36 & 0 & 36 & 36 & 1 & 36 & 1301.5599 & 26 & 10 \\
\hline 4 & 1 & 4 & 4 & 0 & 4 & 1301.5911 & 5 & \\
\hline 35 & 1 & 35 & 35 & 0 & 35 & 1301.6144 & 23 & \\
\hline 32 & 2 & 31 & 32 & 1 & 31 & 1301.6236 & -12 & 10 \\
\hline 7 & 3 & 5 & 8 & 2 & 7 & 1301.6236 & 1 & 10 \\
\hline 3 & 1 & 3 & 3 & 0 & 3 & 1301.6465 & 6 & \\
\hline 2 & 1 & 2 & 2 & 0 & 2 & 1301.6888 & 5 & \\
\hline
\end{tabular}

\begin{tabular}{|c|c|c|c|c|c|c|c|}
\hline 1 & 1 & 1 & 1 & 0 & 1 & 1301.7175 & 6 \\
\hline 22 & 7 & 15 & 23 & 6 & 17 & 1301.7726 & -4 \\
\hline 36 & 1 & 36 & 36 & 0 & 36 & 1301.7852 & 25 \\
\hline 31 & 2 & 30 & 31 & 1 & 30 & 1301.8044 & -8 \\
\hline 8 & 1 & 7 & 7 & 2 & 5 & 1301.9381 & 3 \\
\hline 30 & 2 & 29 & 30 & 1 & 29 & 1302.0034 & -13 \\
\hline 29 & 2 & 28 & 29 & 1 & 28 & 1302.2207 & -11 \\
\hline 18 & 6 & 12 & 19 & 5 & 14 & 1302.2433 & 11 \\
\hline 9 & 1 & 9 & 8 & 2 & 7 & 1302.3506 & 4 \\
\hline 17 & 3 & 15 & 16 & 4 & 13 & 1302.3682 & 0 \\
\hline 17 & 3 & 14 & 16 & 4 & 12 & 1302.4018 & -2 \\
\hline 28 & 2 & 27 & 28 & 1 & 27 & 1302.4538 & -11 \\
\hline 14 & 5 & 9 & 15 & 4 & 11 & 1302.6370 & 3 \\
\hline 13 & 2 & 12 & 12 & 3 & 10 & 1302.6500 & -4 \\
\hline 27 & 2 & 26 & 27 & 1 & 26 & 1302.7009 & -10 \\
\hline 26 & 5 & 21 & 25 & 6 & 19 & 1302.9052 & -15 \\
\hline 26 & 5 & 22 & 25 & 6 & 20 & 1302.9140 & 6 \\
\hline 13 & 2 & 11 & 12 & 3 & 9 & 1302.9188 & 1 \\
\hline 10 & 4 & 6 & 11 & 3 & 8 & 1302.9447 & 4 \\
\hline 10 & 4 & 7 & 11 & 3 & 9 & 1302.9504 & 13 \\
\hline 26 & 2 & 25 & 26 & 1 & 25 & 1302.9598 & -10 \\
\hline 5 & 0 & 5 & 4 & 1 & 3 & 1303.0238 & 5 \\
\hline 6 & 3 & 3 & 7 & 2 & 5 & 1303.1445 & -3 \\
\hline 6 & 3 & 4 & 7 & 2 & 6 & 1303.1831 & 1 \\
\hline 25 & 2 & 24 & 25 & 1 & 24 & 1303.2285 & -8 \\
\hline 1 & 1 & 0 & 0 & 0 & 0 & 1303.3148 & 6 \\
\hline 21 & 7 & 14 & 22 & 6 & 16 & 1303.3324 & 6 \\
\hline 22 & 4 & 19 & 21 & 5 & 17 & 1303.4744 & 2 \\
\hline 22 & 4 & 18 & 21 & 5 & 16 & 1303.4789 & -4 \\
\hline 24 & 2 & 23 & 24 & 1 & 23 & 1303.5041 & -10 \\
\hline 9 & 1 & 8 & 8 & 2 & 6 & 1303.6117 & -2 \\
\hline 10 & 1 & 10 & 9 & 2 & 8 & 1303.7697 & 0 \\
\hline 23 & 2 & 22 & 23 & 1 & 22 & 1303.7852 & -7 \\
\hline 17 & 6 & 11 & 18 & 5 & 13 & 1303.8060 & 2 \\
\hline 18 & 3 & 16 & 17 & 4 & 14 & 1303.9392 & -3 \\
\hline 18 & 3 & 15 & 17 & 4 & 13 & 1303.9864 & -1 \\
\hline 22 & 2 & 21 & 22 & 1 & 21 & 1304.0688 & -7 \\
\hline 14 & 2 & 13 & 13 & 3 & 11 & 1304.1924 & -1 \\
\hline 13 & 5 & 8 & 14 & 4 & 10 & 1304.2037 & 0 \\
\hline 21 & 2 & 20 & 21 & 1 & 20 & 1304.3532 & -6 \\
\hline 6 & 0 & 6 & 5 & 1 & 4 & 1304.4965 & 5 \\
\hline 9 & 4 & 5 & 10 & 3 & 7 & 1304.5155 & 10 \\
\hline 14 & 2 & 12 & 13 & 3 & 10 & 1304.5458 & -1 \\
\hline 20 & 2 & 19 & 20 & 1 & 19 & 1304.6360 & -6 \\
\hline 5 & 3 & 2 & 6 & 2 & 4 & 1304.7240 & 3 \\
\hline 5 & 3 & 3 & 6 & 2 & 5 & 1304.7452 & 2 \\
\hline 20 & 7 & 13 & 21 & 6 & 15 & 1304.8906 & 2 \\
\hline 19 & 2 & 18 & 19 & 1 & 18 & 1304.9146 & -15 \\
\hline 23 & 4 & 20 & 22 & 5 & 18 & 1305.0480 & 7 \\
\hline 23 & 4 & 19 & 22 & 5 & 17 & 1305.0551 & 5 \\
\hline 11 & 1 & 11 & 10 & 2 & 9 & 1305.1743 & 4 \\
\hline 18 & 2 & 17 & 18 & 1 & 17 & 1305.1898 & -6 \\
\hline 10 & 1 & 9 & 9 & 2 & 7 & 1305.2891 & 0 \\
\hline 16 & 6 & 10 & 17 & 5 & 12 & 1305.3680 & -9 \\
\hline 17 & 2 & 16 & 17 & 1 & 16 & 1305.4574 & -5 \\
\hline 19 & 3 & 17 & 18 & 4 & 15 & 1305.5095 & -3 \\
\hline 19 & 3 & 16 & 18 & 4 & 14 & 1305.5734 & -5 \\
\hline 16 & 2 & 15 & 16 & 1 & 15 & 1305.7168 & -4 \\
\hline 15 & 2 & 14 & 14 & 3 & 12 & 1305.7278 & -5 \\
\hline 12 & 5 & 7 & 13 & 4 & 9 & 1305.7699 & -2 \\
\hline 7 & 0 & 7 & 6 & 1 & 5 & 1305.9479 & 3 \\
\hline 15 & 2 & 14 & 15 & 1 & 14 & 1305.9665 & -3 \\
\hline 8 & 4 & 4 & 9 & 3 & 6 & 1306.0843 & 8 \\
\hline 15 & 2 & 13 & 14 & 3 & 11 & 1306.1837 & -1 \\
\hline 14 & 2 & 13 & 14 & 1 & 13 & 1306.2052 & -3 \\
\hline 13 & 2 & 12 & 13 & 1 & 12 & 1306.4320 & -3 \\
\hline 19 & 7 & 12 & 20 & 6 & 14 & 1306.4499 & 11 \\
\hline 3 & 1 & 2 & 2 & 0 & 2 & 1306.5256 & 7 \\
\hline 12 & 1 & 12 & 11 & 2 & 10 & 1306.5630 & 0 \\
\hline 24 & 4 & 21 & 23 & 5 & 19 & 1306.6203 & 3 \\
\hline 24 & 4 & 20 & 23 & 5 & 18 & 1306.6304 & 2 \\
\hline 12 & 2 & 11 & 12 & 1 & 11 & 1306.6460 & -2 \\
\hline 11 & 2 & 10 & 11 & 1 & 10 & 1306.8464 & 1 \\
\hline 15 & 6 & 9 & 16 & 5 & 11 & 1306.9319 & 1 \\
\hline 11 & 1 & 10 & 10 & 2 & 8 & 1306.9668 & -1 \\
\hline 10 & 2 & 9 & 10 & 1 & 9 & 1307.0320 & -1 \\
\hline 20 & 3 & 18 & 19 & 4 & 16 & 1307.0791 & \\
\hline
\end{tabular}




\begin{tabular}{|c|c|c|c|c|c|c|c|}
\hline 20 & 3 & 17 & 19 & 4 & 15 & 1307.1645 & -2 \\
\hline 9 & 2 & 8 & 9 & 1 & 8 & 1307.2028 & 1 \\
\hline 16 & 2 & 15 & 15 & 3 & 13 & 1307.2570 & 0 \\
\hline 11 & 5 & 6 & 12 & 4 & 8 & 1307.3361 & 1 \\
\hline 8 & 2 & 7 & 8 & 1 & 7 & 1307.3579 & 3 \\
\hline 8 & 0 & 8 & 7 & 1 & 6 & 1307.3773 & 4 \\
\hline 7 & 2 & 6 & 7 & 1 & 6 & 1307.4968 & 4 \\
\hline 6 & 2 & 5 & 6 & 1 & 5 & 1307.6188 & 1 \\
\hline 7 & 4 & 3 & 8 & 3 & 5 & 1307.6519 & 4 \\
\hline 5 & 2 & 4 & 5 & 1 & 4 & 1307.7246 & 5 \\
\hline 4 & 2 & 3 & 4 & 1 & 3 & 1307.8130 & 7 \\
\hline 16 & 2 & 14 & 15 & 3 & 12 & 1307.8326 & -5 \\
\hline 3 & 2 & 2 & 3 & 1 & 2 & 1307.8838 & 6 \\
\hline 13 & 1 & 13 & 12 & 2 & 11 & 1307.9369 & 1 \\
\hline 2 & 2 & 1 & 2 & 1 & 1 & 1307.9369 & 4 \\
\hline 18 & 7 & 11 & 19 & 6 & 13 & 1308.0084 & 13 \\
\hline 2 & 2 & 0 & 2 & 1 & 2 & 1308.0456 & 5 \\
\hline 3 & 2 & 1 & 3 & 1 & 3 & 1308.1015 & 4 \\
\hline 4 & 1 & 3 & 3 & 0 & 3 & 1308.1537 & 4 \\
\hline 4 & 2 & 2 & 4 & 1 & 4 & 1308.1775 & 6 \\
\hline 25 & 4 & 22 & 24 & 5 & 20 & 1308.1905 & -13 \\
\hline 25 & 4 & 21 & 24 & 5 & 19 & 1308.2063 & 1 \\
\hline 5 & 2 & 3 & 5 & 1 & 5 & 1308.2738 & 4 \\
\hline 32 & 3 & 30 & 32 & 2 & 30 & 1308.3541 & -7 \\
\hline 6 & 2 & 4 & 6 & 1 & 6 & 1308.3922 & 3 \\
\hline 14 & 6 & 8 & 15 & 5 & 10 & 1308.4944 & 0 \\
\hline 7 & 2 & 5 & 7 & 1 & 7 & 1308.5341 & 3 \\
\hline 12 & 1 & 11 & 11 & 2 & 9 & 1308.6422 & 0 \\
\hline 8 & 2 & 6 & 8 & 1 & 8 & 1308.7010 & 2 \\
\hline 21 & 3 & 18 & 20 & 4 & 16 & 1308.7594 & -1 \\
\hline 17 & 2 & 16 & 16 & 3 & 14 & 1308.7777 & -2 \\
\hline 9 & 0 & 9 & 8 & 1 & 7 & 1308.7832 & 1 \\
\hline 31 & 3 & 29 & 31 & 2 & 29 & 1308.7997 & -21 \\
\hline 9 & 2 & 7 & 9 & 1 & 9 & 1308.8947 & 1 \\
\hline 10 & 5 & 5 & 11 & 4 & 7 & 1308.9022 & 6 \\
\hline 10 & 2 & 8 & 10 & 1 & 10 & 1309.1172 & -1 \\
\hline 6 & 4 & 2 & 7 & 3 & 4 & 1309.2189 & -1 \\
\hline 30 & 3 & 28 & 30 & 2 & 28 & 1309.2389 & -11 \\
\hline 14 & 1 & 14 & 13 & 2 & 12 & 1309.2957 & 3 \\
\hline 11 & 2 & 9 & 11 & 1 & 11 & 1309.3709 & -1 \\
\hline 17 & 2 & 15 & 16 & 3 & 13 & 1309.4934 & -6 \\
\hline 17 & 7 & 10 & 18 & 6 & 12 & 1309.5667 & 12 \\
\hline 12 & 2 & 10 & 12 & 1 & 12 & 1309.6578 & -2 \\
\hline 29 & 3 & 27 & 29 & 2 & 27 & 1309.6668 & -7 \\
\hline 26 & 4 & 23 & 25 & 5 & 21 & 1309.7635 & 12 \\
\hline 26 & 4 & 22 & 25 & 5 & 20 & 1309.7836 & 11 \\
\hline 5 & 1 & 4 & 4 & 0 & 4 & 1309.7982 & 3 \\
\hline 13 & 2 & 11 & 13 & 1 & 13 & 1309.9803 & -2 \\
\hline 13 & 6 & 7 & 14 & 5 & 9 & 1310.0570 & 0 \\
\hline 28 & 3 & 26 & 28 & 2 & 26 & 1310.0813 & -8 \\
\hline 10 & 0 & 10 & 9 & 1 & 8 & 1310.1651 & 2 \\
\hline 22 & 3 & 20 & 21 & 4 & 18 & 1310.2102 & -8 \\
\hline 18 & 2 & 17 & 17 & 3 & 15 & 1310.2899 & -4 \\
\hline 13 & 1 & 12 & 12 & 2 & 10 & 1310.3115 & -6 \\
\hline 14 & 2 & 12 & 14 & 1 & 14 & 1310.3406 & -4 \\
\hline 22 & 3 & 19 & 21 & 4 & 17 & 1310.3586 & -1 \\
\hline 9 & 5 & 4 & 10 & 4 & 6 & 1310.4672 & 3 \\
\hline 27 & 3 & 25 & 27 & 2 & 25 & 1310.4811 & -9 \\
\hline 20 & 8 & 12 & 21 & 7 & 14 & 1310.5597 & 29 \\
\hline 15 & 1 & 15 & 14 & 2 & 13 & 1310.6390 & 1 \\
\hline 15 & 2 & 13 & 15 & 1 & 15 & 1310.7415 & -3 \\
\hline 5 & 4 & 1 & 6 & 3 & 3 & 1310.7863 & 3 \\
\hline 26 & 3 & 24 & 26 & 2 & 24 & 1310.8649 & -5 \\
\hline 2 & 2 & 0 & 1 & 1 & 0 & 1311.1089 & 5 \\
\hline 16 & 7 & 9 & 17 & 6 & 11 & 1311.1247 & 7 \\
\hline 2 & 2 & 1 & 1 & 1 & 1 & 1311.1448 & 4 \\
\hline 18 & 2 & 16 & 17 & 3 & 14 & 1311.1662 & -3 \\
\hline 16 & 2 & 14 & 16 & 1 & 16 & 1311.1844 & -5 \\
\hline 25 & 3 & 23 & 25 & 2 & 23 & 1311.2299 & -8 \\
\hline 27 & 4 & 24 & 26 & 5 & 22 & 1311.3296 & 9 \\
\hline 27 & 4 & 23 & 26 & 5 & 21 & 1311.3597 & 3 \\
\hline 6 & 1 & 5 & 5 & 0 & 5 & 1311.4598 & 3 \\
\hline 11 & 0 & 11 & 10 & 1 & 9 & 1311.5212 & 0 \\
\hline 24 & 3 & 22 & 24 & 2 & 22 & 1311.5756 & -6 \\
\hline 12 & 6 & 6 & 13 & 5 & 8 & 1311.6194 & -2 \\
\hline 17 & 2 & 15 & 17 & 1 & 17 & 1311.6720 & -5 \\
\hline 23 & 3 & 21 & 22 & 4 & 19 & 1311.7724 & -10 \\
\hline
\end{tabular}

$\begin{array}{lll}1311.7927 & -5 & 10\end{array}$ $1311.9648 \quad 15 \quad 4$ $1311.9672 \quad-2 \quad 4$ $1311.9731-3 \quad 4$ $1312.0334 \quad 12 \quad 4$ $1312.1110 \quad 10 \quad 10$ \begin{tabular}{rrr}
1312.4813 & -4 & 4 \\
\hline
\end{tabular} $1312.6422 \quad 6 \quad 4$ $\begin{array}{rrr}1312.6422 & 6 & 4 \\ 1312.6830 & 3 & 4\end{array}$ $1312.7362 \quad-4 \quad 4$ $\begin{array}{rrr}1312.7495 & 5 & 4 \\ 1312.7876 & -6 & 4\end{array}$ $1312.8506 \quad-3 \quad 10$ $1312.8506 \quad 3 \quad 10$ $\begin{array}{lll}1312.9669 & -2 & 4\end{array}$ $\begin{array}{lll}1313.1394 & 1 & 4\end{array}$ $1313.1728 \quad-3 \quad 4$ $\begin{array}{lll}1313.1821 & -1 & 4\end{array}$ $1313.2808 \quad-2 \quad 4$ $1313.2863 \quad 1 \quad 4$ $1313.3319 \quad-8 \quad 4$ $\begin{array}{lll}1313.3546 & -4 & 4\end{array}$ $1313.4188 \quad-5 \quad 4$ $\begin{array}{lll}1313.5128 & -4 & 4\end{array}$ $\begin{array}{lll}1313.5733 & -4 & 4\end{array}$ $\begin{array}{lll}1313.5973 & -3 & 4 \\ 1313.6224 & -6 & 4\end{array}$ $\begin{array}{lll}1313.6485 & -3 & 4\end{array}$ $1313.6656 \quad 21 \quad 10$ $1313.7630 \quad-1 \quad 4$ $\begin{array}{lll}1313.8575 & -2 & 4\end{array}$ 1313.9953 0 4 $1314.0422 \quad 0 \quad 4$ $\begin{array}{lll}1314.0776 & 3 & 4\end{array}$ $\begin{array}{lll}1314.1023 & -4 & 4\end{array}$ $1314.1202 \quad 1 \quad 4$ $\begin{array}{rrr}1314.1316 & 3 & 4\end{array}$ $1314.1588 \quad 2 \quad 10$ $1314.1588 \quad 8 \quad 10$ $\begin{array}{lll}1314.1671 & 2 & 4\end{array}$ $\begin{array}{lll}1314.1784 & 3 & 4\end{array}$ $\begin{array}{lll}1314.1933 & 2 & 4\end{array}$ $1314.2128 \quad 1 \quad 4$ $\begin{array}{lll}1314.2379 & 1 & 4\end{array}$ $1314.2692 \quad-2 \quad 4$ $\begin{array}{lll}1314.3084 & -2 & 4\end{array}$ $\begin{array}{lll}1314.3564 & -3 & 4\end{array}$ $\begin{array}{rrr}1314.3722 & 4 & 4 \\ 1314.4148 & -2 & 4\end{array}$ $\begin{array}{lll}1314.4845 & -5 & 4\end{array}$ $\begin{array}{lll}1314.5444 & -3 & 4\end{array}$ $\begin{array}{lll}1314.5681 & -2 & 4\end{array}$ $\begin{array}{lll}1314.5795 & -3 & 4\end{array}$ $\begin{array}{lll}1314.6662 & -4 & 4\end{array}$ $1314.7452 \quad 1 \quad 4$ $\begin{array}{lll}1314.7678 & -5 & 4\end{array}$ $\begin{array}{lll}1314.7815 & -2 & 4\end{array}$ $\begin{array}{lll}1314.8322 & -11 & 4\end{array}$ $\begin{array}{rrr}1314.8876 & -7 & 4\end{array}$ $\begin{array}{lll}1314.9156 & -1 & 4\end{array}$ $\begin{array}{lll}1315.0703 & -2 & 4\end{array}$ $\begin{array}{lrr}1315.1907 & 0 & 4 \\ 1315.2187 & 13 & 10\end{array}$ $\begin{array}{rrr}1315.2487 & 2 & 4\end{array}$ $\begin{array}{rrr}1315.2572 & -7 & 4\end{array}$ $1315.4260 \quad 0 \quad 4$ $\begin{array}{lll}1315.4523 & 5 & 4\end{array}$ $\begin{array}{lll}1315.6168 & -4 & 4\end{array}$ $\begin{array}{rrr}1315.6590 & 4 & 4 \\ 1315.6833 & 3 & 4\end{array}$ $\begin{array}{lll}1315.8005 & -5 & 4\end{array}$ $\begin{array}{lll}1315.8637 & -5 & 4\end{array}$ 


\begin{tabular}{|c|c|c|c|}
\hline 26 & 3 & 23 & 26 \\
\hline 5 & 2 & 4 & 4 \\
\hline 22 & 2 & 21 & 21 \\
\hline 21 & 2 & 19 & 20 \\
\hline 9 & 6 & 3 & 10 \\
\hline 26 & 3 & 24 & 25 \\
\hline 24 & 2 & 22 & 24 \\
\hline 9 & 1 & 8 & 8 \\
\hline 28 & 3 & 25 & 28 \\
\hline 15 & 0 & 15 & 14 \\
\hline 16 & 8 & 8 & 17 \\
\hline 26 & 3 & 23 & 25 \\
\hline 17 & 1 & 16 & 16 \\
\hline 29 & 3 & 26 & 29 \\
\hline 20 & 1 & 20 & 19 \\
\hline 6 & 2 & 4 & 5 \\
\hline 25 & 2 & 23 & 25 \\
\hline 30 & 3 & 27 & 30 \\
\hline 12 & 7 & 5 & 13 \\
\hline 6 & 2 & 5 & 5 \\
\hline 23 & 2 & 22 & 22 \\
\hline 16 & 0 & 16 & 15 \\
\hline 22 & 2 & 20 & 21 \\
\hline 27 & 3 & 25 & 26 \\
\hline 26 & 2 & 24 & 26 \\
\hline 10 & 1 & 9 & 9 \\
\hline 15 & 8 & 7 & 16 \\
\hline 21 & 1 & 21 & 20 \\
\hline 18 & 1 & 17 & 17 \\
\hline 7 & 2 & 5 & 6 \\
\hline 3 & 3 & 0 & 2 \\
\hline 11 & 7 & 4 & 12 \\
\hline 17 & 0 & 17 & 16 \\
\hline 24 & 2 & 23 & 23 \\
\hline 27 & 4 & 24 & 27 \\
\hline 7 & 2 & 6 & 6 \\
\hline 26 & 4 & 23 & 26 \\
\hline 25 & 4 & 22 & 25 \\
\hline 22 & 1 & 22 & 21 \\
\hline 24 & 4 & 21 & 24 \\
\hline 23 & 2 & 21 & 22 \\
\hline 23 & 4 & 20 & 23 \\
\hline 22 & 4 & 19 & 22 \\
\hline 21 & 4 & 18 & 21 \\
\hline 20 & 4 & 17 & 20 \\
\hline 19 & 4 & 16 & 19 \\
\hline 19 & 1 & 18 & 18 \\
\hline 18 & 4 & 15 & 18 \\
\hline 11 & 1 & 10 & 10 \\
\hline 8 & 2 & 6 & 7 \\
\hline 25 & 4 & 21 & 25 \\
\hline 17 & 4 & 14 & 17 \\
\hline 24 & 4 & 20 & 24 \\
\hline 23 & 4 & 19 & 23 \\
\hline 22 & 4 & 18 & 22 \\
\hline 16 & 4 & 13 & 16 \\
\hline 21 & 4 & 17 & 21 \\
\hline 20 & 4 & 16 & 20 \\
\hline 15 & 4 & 12 & 15 \\
\hline 19 & 4 & 15 & 19 \\
\hline 18 & 4 & 14 & 18 \\
\hline 17 & 4 & 13 & 17 \\
\hline 14 & 4 & 11 & 14 \\
\hline 16 & 4 & 12 & 16 \\
\hline 15 & 4 & 11 & 15 \\
\hline 13 & 4 & 10 & 13 \\
\hline 12 & 4 & 9 & 12 \\
\hline 14 & 4 & 10 & 14 \\
\hline 18 & 0 & 18 & 17 \\
\hline 4 & 3 & 1 & 3 \\
\hline 25 & 2 & 24 & 24 \\
\hline 23 & 1 & 23 & 22 \\
\hline 8 & 2 & 7 & 7 \\
\hline 19 & 0 & 19 & 18 \\
\hline 24 & 2 & 22 & 23 \\
\hline 13 & 8 & 5 & 14 \\
\hline 9 & 2 & 7 & \\
\hline
\end{tabular}

\begin{tabular}{|c|c|c|c|c|}
\hline 2 & 25 & 1315.9452 & 6 & 4 \\
\hline 1 & 4 & 1316.0127 & -1 & 4 \\
\hline 3 & 19 & 1316.2388 & -3 & 10 \\
\hline 3 & 17 & 1316.2479 & -4 & 4 \\
\hline 5 & 5 & 1316.3084 & 2 & 4 \\
\hline 4 & 22 & 1316.4391 & -5 & 4 \\
\hline 1 & 24 & 1316.4532 & 3 & 4 \\
\hline 0 & 8 & 1316.5591 & 0 & 4 \\
\hline 2 & 27 & 1316.5703 & 17 & 4 \\
\hline 1 & 13 & 1316.6696 & 0 & 4 \\
\hline 7 & 10 & 1316.7729 & 11 & 10 \\
\hline 4 & 21 & 1316.8159 & 9 & 4 \\
\hline 2 & 14 & 1316.8742 & -8 & 4 \\
\hline 2 & 28 & 1316.9384 & 21 & 4 \\
\hline 2 & 18 & 1317.1342 & -1 & 4 \\
\hline 1 & 4 & 1317.1449 & 3 & 4 \\
\hline 1 & 25 & 1317.3412 & 12 & 4 \\
\hline 2 & 29 & 1317.3465 & 21 & 4 \\
\hline 6 & 7 & 1317.3603 & -3 & 4 \\
\hline 1 & 5 & 1317.6726 & 4 & 4 \\
\hline 3 & 20 & 1317.6971 & -7 & 4 \\
\hline 1 & 14 & 1317.8827 & -2 & 4 \\
\hline 3 & 18 & 1317.9598 & 1 & 4 \\
\hline 4 & 23 & 1317.9853 & -8 & 4 \\
\hline 1 & 26 & 1318.2795 & 11 & 4 \\
\hline 0 & 9 & 1318.3021 & -3 & 4 \\
\hline 7 & 9 & 1318.3279 & 11 & 10 \\
\hline 2 & 19 & 1318.3905 & 1 & 4 \\
\hline 2 & 15 & 1318.4711 & -7 & 4 \\
\hline 1 & 5 & 1318.6175 & 3 & 4 \\
\hline 2 & 0 & 1318.8477 & 5 & 4 \\
\hline 6 & 6 & 1318.9206 & -1 & 4 \\
\hline 1 & 15 & 1319.0655 & 0 & 4 \\
\hline 3 & 21 & 1319.1428 & -12 & 4 \\
\hline 3 & 24 & 1319.2163 & 16 & 4 \\
\hline 1 & 6 & 1319.3502 & 2 & 4 \\
\hline 3 & 23 & 1319.3827 & 6 & 4 \\
\hline 3 & 22 & 1319.5258 & 11 & 4 \\
\hline 2 & 20 & 1319.6336 & 6 & 4 \\
\hline 3 & 21 & 1319.6481 & 12 & 4 \\
\hline 3 & 19 & 1319.6769 & -1 & 4 \\
\hline 3 & 20 & 1319.7512 & 2 & 4 \\
\hline 3 & 19 & 1319.8390 & 0 & 4 \\
\hline 3 & 18 & 1319.9132 & 5 & 4 \\
\hline 3 & 17 & 1319.9741 & 1 & 4 \\
\hline 3 & 16 & 1320.0241 & -2 & 4 \\
\hline 2 & 16 & 1320.0448 & -8 & 4 \\
\hline 3 & 15 & 1320.0653 & 1 & 4 \\
\hline 0 & 10 & 1320.0704 & 0 & 4 \\
\hline 1 & 6 & 1320.0785 & 3 & 4 \\
\hline 3 & 23 & 1320.0988 & -4 & 10 \\
\hline 3 & 14 & 1320.0988 & 7 & 10 \\
\hline 3 & 22 & 1320.1038 & 15 & 4 \\
\hline 3 & 21 & 1320.1083 & 2 & 4 \\
\hline 3 & 20 & 1320.1154 & -2 & 4 \\
\hline 3 & 13 & 1320.1242 & 0 & 10 \\
\hline 3 & 19 & 1320.1242 & 0 & 10 \\
\hline 3 & 18 & 1320.1330 & -4 & 4 \\
\hline 3 & 12 & 1320.1437 & -8 & 10 \\
\hline 3 & 17 & 1320.1437 & 11 & 10 \\
\hline 3 & 16 & 1320.1517 & 1 & 4 \\
\hline 3 & 15 & 1320.1599 & -1 & 10 \\
\hline 3 & 11 & 1320.1599 & -2 & 10 \\
\hline 3 & 14 & 1320.1683 & 7 & 4 \\
\hline 3 & 13 & 1320.1729 & -14 & 10 \\
\hline 3 & 10 & 1320.1729 & 12 & 10 \\
\hline 3 & 9 & 1320.1801 & 0 & 10 \\
\hline 3 & 12 & 1320.1801 & 1 & 10 \\
\hline 1 & 16 & 1320.2173 & -1 & 4 \\
\hline 2 & 1 & 1320.4160 & 11 & 4 \\
\hline 3 & 22 & 1320.5757 & -13 & 4 \\
\hline 2 & 21 & 1320.8632 & 9 & 4 \\
\hline 1 & 7 & 1321.0465 & 1 & 4 \\
\hline 1 & 17 & 1321.3388 & -1 & 4 \\
\hline 3 & 20 & 1321.3987 & 9 & 4 \\
\hline I & 7 & 1321.4379 & -7 & 10 \\
\hline 1 & 7 & 1321.5295 & 1 & 4 \\
\hline
\end{tabular}

\begin{tabular}{|c|c|c|c|c|c|c|c|}
\hline 20 & 1 & 19 & 19 & 2 & 17 & 1321.5934 & -8 \\
\hline 12 & 1 & 11 & 11 & 0 & 11 & 1321.8649 & -2 \\
\hline 5 & 3 & 2 & 4 & 2 & 2 & 1321.9821 & 7 \\
\hline 5 & 3 & 3 & 4 & 2 & 3 & 1321.9859 & -1 \\
\hline 26 & 2 & 25 & 25 & 3 & 23 & 1321.9968 & 3 \\
\hline 24 & 1 & 24 & 23 & 2 & 22 & 1322.0801 & 11 \\
\hline 20 & 0 & 20 & 19 & 1 & 18 & 1322.4303 & -1 \\
\hline 9 & 2 & 8 & 8 & 1 & 8 & 1322.7615 & 0 \\
\hline 10 & 2 & 8 & 9 & 1 & 8 & 1322.9729 & 1 \\
\hline 21 & 1 & 20 & 20 & 2 & 18 & 1323.1152 & -6 \\
\hline 25 & 2 & 23 & 24 & 3 & 21 & 1323.1201 & 7 \\
\hline 25 & 1 & 25 & 24 & 2 & 23 & 1323.2836 & 3 \\
\hline 27 & 2 & 26 & 26 & 3 & 24 & 1323.4003 & -17 \\
\hline 21 & 0 & 21 & 20 & 1 & 19 & 1323.4933 & 1 \\
\hline 6 & 3 & 3 & 5 & 2 & 3 & 1323.5463 & 5 \\
\hline 6 & 3 & 4 & 5 & 2 & 4 & 1323.5571 & 7 \\
\hline 13 & 1 & 12 & 12 & 0 & 12 & 1323.6881 & -4 \\
\hline 11 & 2 & 9 & 10 & 1 & 9 & 1324.4109 & 1 \\
\hline 26 & 1 & 26 & 25 & 2 & 24 & 1324.4757 & -2 \\
\hline 10 & 2 & 9 & 9 & 1 & 9 & 1324.4954 & 0 \\
\hline 22 & 0 & 22 & 21 & 1 & 20 & 1324.5283 & -3 \\
\hline 22 & 1 & 21 & 21 & 2 & 19 & 1324.6080 & -5 \\
\hline 28 & 2 & 27 & 27 & 3 & 25 & 1324.7929 & -3 \\
\hline 26 & 2 & 24 & 25 & 3 & 22 & 1324.8394 & 4 \\
\hline 7 & 3 & 4 & 6 & 2 & 4 & 1325.1070 & 1 \\
\hline 7 & 3 & 5 & 6 & 2 & 5 & 1325.1283 & 2 \\
\hline 14 & 1 & 13 & 13 & 0 & 13 & 1325.5413 & -11 \\
\hline 32 & 5 & 28 & 32 & 4 & 28 & 1325.5792 & -1 \\
\hline 30 & 5 & 26 & 30 & 4 & 26 & 1325.7398 & 6 \\
\hline 29 & 5 & 25 & 29 & 4 & 25 & 1325.8044 & 3 \\
\hline 12 & 2 & 10 & 11 & 1 & 10 & 1325.8458 & -1 \\
\hline 28 & 5 & 24 & 28 & 4 & 24 & 1325.8609 & 4 \\
\hline 29 & 5 & 24 & 29 & 4 & 26 & 1325.8859 & -31 \\
\hline 27 & 5 & 23 & 27 & 4 & 23 & 1325.9094 & 2 \\
\hline 28 & 5 & 23 & 28 & 4 & 25 & 1325.9215 & -23 \\
\hline 27 & 5 & 22 & 27 & 4 & 24 & 1325.9520 & -35 \\
\hline 26 & 5 & 22 & 26 & 4 & 22 & 1325.9520 & 9 \\
\hline 26 & 5 & 21 & 26 & 4 & 23 & 1325.9835 & -5 \\
\hline 25 & 5 & 21 & 25 & 4 & 21 & 1325.9877 & 6 \\
\hline 25 & 5 & 20 & 25 & 4 & 22 & 1326.0086 & -8 \\
\hline 24 & 5 & 20 & 24 & 4 & 20 & 1326.0179 & 3 \\
\hline 24 & 5 & 19 & 24 & 4 & 21 & 1326.0312 & -3 \\
\hline 23 & 5 & 19 & 23 & 4 & 19 & 1326.0432 & -3 \\
\hline 23 & 5 & 18 & 23 & 4 & 20 & 1326.0501 & 4 \\
\hline 22 & 5 & 17 & 22 & 4 & 19 & 1326.0635 & 24 \\
\hline 23 & 1 & 22 & 22 & 2 & 20 & 1326.0702 & -7 \\
\hline 21 & 5 & 17 & 21 & 4 & 17 & 1326.0832 & 0 \\
\hline 20 & 5 & 16 & 20 & 4 & 16 & 1326.0979 & -1 \\
\hline 20 & 5 & 15 & 20 & 4 & 17 & 1326.1091 & 2 \\
\hline 21 & 5 & 16 & 21 & 4 & 18 & 1326.1169 & -10 \\
\hline 17 & 5 & 13 & 17 & 4 & 13 & 1326.1282 & 13 \\
\hline 29 & 2 & 28 & 28 & 3 & 26 & 1326.1693 & -5 \\
\hline 11 & 2 & 10 & 10 & 1 & 10 & 1326.2484 & 0 \\
\hline 4 & 4 & 0 & 3 & 3 & 0 & 1326.4621 & 0 \\
\hline 24 & 0 & 24 & 23 & 1 & 22 & 1326.5245 & -4 \\
\hline 27 & 2 & 25 & 26 & 3 & 23 & 1326.5544 & 11 \\
\hline 8 & 3 & 5 & 7 & 2 & 5 & 1326.6635 & 1 \\
\hline 8 & 3 & 6 & 7 & 2 & 6 & 1326.7017 & 3 \\
\hline 28 & 1 & 28 & 27 & 2 & 26 & 1326.8288 & 8 \\
\hline 13 & 2 & 11 & 12 & 1 & 11 & 1327.2804 & -2 \\
\hline 15 & 1 & 14 & 14 & 0 & 14 & 1327.4279 & -8 \\
\hline 25 & 0 & 25 & 24 & 1 & 23 & 1327.4910 & 6 \\
\hline 24 & 1 & 23 & 23 & 2 & 21 & 1327.5011 & -6 \\
\hline 12 & 2 & 11 & 11 & 1 & 11 & 1328.0206 & 0 \\
\hline 5 & 4 & 1 & 4 & 3 & 1 & 1328.0307 & 1 \\
\hline 9 & 3 & 6 & 8 & 2 & 6 & 1328.2139 & 1 \\
\hline 28 & 2 & 26 & 27 & 3 & 24 & 1328.2609 & 17 \\
\hline 9 & 3 & 7 & 8 & 2 & 7 & 1328.2770 & 2 \\
\hline 26 & 0 & 26 & 25 & 1 & 24 & 1328.4385 & 8 \\
\hline 14 & 2 & 12 & 13 & 1 & 12 & 1328.7174 & -4 \\
\hline 25 & 1 & 24 & 24 & 2 & 22 & 1328.8996 & -5 \\
\hline 16 & 1 & 15 & 15 & 0 & 15 & 1329.3483 & -6 \\
\hline 27 & 0 & 27 & 26 & 1 & 25 & 1329.3698 & 1 \\
\hline 6 & 4 & 2 & 5 & 3 & 2 & 1329.5991 & 1 \\
\hline 10 & 3 & 7 & 9 & 2 & 7 & 1329.7564 & -1 \\
\hline 13 & 2 & 12 & 12 & 1 & 12 & 1329.8119 & -3 \\
\hline 10 & 3 & 8 & 9 & 2 & 8 & 1329.8550 & 1 \\
\hline
\end{tabular}


1 1 16 11 17 18

19 20

\begin{tabular}{|c|c|c|}
\hline 343,351 & -4 & \\
\hline 1343.3580 & -3 & \\
\hline 1343.3655 & 1 & \\
\hline 1343.3731 & 4 & \\
\hline 1343.3798 & -2 & 10 \\
\hline 1343.3798 & 3 & 10 \\
\hline 1343.3883 & 10 & \\
\hline 1343.3956 & 12 & \\
\hline 1343.4026 & 12 & \\
\hline 1343.4092 & 11 & \\
\hline 1343.4157 & 13 & \\
\hline 1343.4215 & 13 & \\
\hline 1343.4270 & 16 & \\
\hline 1343.6644 & 0 & \\
\hline 1343.6838 & -1 & \\
\hline 1343.7994 & -10 & \\
\hline 1343.8519 & -1 & \\
\hline 1344.2862 & -6 & \\
\hline 1344.3859 & -1 & \\
\hline 1344.5102 & -3 & \\
\hline 1344.8584 & -6 & \\
\hline 1344.9458 & -1 & 4 \\
\hline 1345.2135 & -2 & \\
\hline 1345.2428 & 0 & \\
\hline 1345.5016 & 7 & \\
\hline 1345.7465 & -2 & 4 \\
\hline 1345.9296 & -3 & \\
\hline 1346.0043 & -6 & 4 \\
\hline 1346.0794 & -2 & 4 \\
\hline 1346.5113 & -1 & \\
\hline 1346.7579 & 1 & 4 \\
\hline 1346.8001 & -1 & 4 \\
\hline 1346.8287 & -9 & 4 \\
\hline 1347.0897 & 2 & 4 \\
\hline 1347.1848 & 7 & 4 \\
\hline 1347.5839 & 0 & 4 \\
\hline 1347.6482 & -3 & 4 \\
\hline 1348.0757 & -1 & 4 \\
\hline 1348.2396 & -6 & 4 \\
\hline 1348.2958 & -1 & \\
\hline 1348.3559 & -1 & 4 \\
\hline 1348.4162 & 1 & 4 \\
\hline 1348.6670 & -10 & 4 \\
\hline 1348.8188 & -10 & 4 \\
\hline 1348.8476 & -30 & 10 \\
\hline 1348.8563 & -21 & 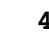 \\
\hline 1348.8652 & -15 & 4 \\
\hline 1348.8743 & -13 & 4 \\
\hline 1348.8843 & -7 & 4 \\
\hline 1348.8950 & 2 & 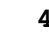 \\
\hline 1348.9051 & 1 & 10 \\
\hline 1348.9051 & 16 & 10 \\
\hline 1348.9161 & 7 & $a_{2}$ \\
\hline 1348.9274 & 13 & 4 \\
\hline 1348.9384 & 14 & 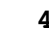 \\
\hline 1348.9498 & 19 & 4 \\
\hline 1348.9610 & 22 & 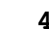 \\
\hline 1348.9715 & 19 & 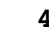 \\
\hline 1348.9823 & 21 & 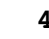 \\
\hline 1348.9914 & 8 & 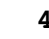 \\
\hline 1349.0010 & 5 & 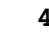 \\
\hline 1349.0088 & -11 & 4 \\
\hline 1349.2169 & -2 & 4 \\
\hline 1349.2492 & -4 & 4 \\
\hline 1349.6389 & 1 & 4 \\
\hline 1349.7289 & 4 & 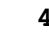 \\
\hline 1349.8268 & 0 & 4 \\
\hline 1349.9101 & -4 & 4 \\
\hline 1350.2370 & -9 & - $>3$ \\
\hline 1350.5028 & -13 & 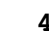 \\
\hline 1350.6626 & 15 & 4 \\
\hline 1350.7850 & -2 & \\
\hline 1350.8292 & -3 & 4 \\
\hline 1350.9270 & -9 & 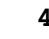 \\
\hline 1351.0296 & 3 & \\
\hline 1351.2003 & -1 & \\
\hline 1351.3494 & 1 & \\
\hline
\end{tabular}




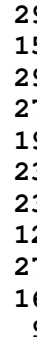

15
29
27
1
2
2
1
2
1

\begin{tabular}{|c|c|c|c|c|c|c|}
\hline 2 & 28 & 28 & 1 & 28 & 1361.1543 & -22 \\
\hline 7 & 8 & 14 & 6 & 8 & 1361.2240 & 3 \\
\hline 3 & 27 & 28 & 2 & 27 & 1361.3010 & -12 \\
\hline 4 & 23 & 26 & 3 & 23 & 1361.6534 & 11 \\
\hline 6 & 13 & 18 & 5 & 13 & 1361.7379 & 3 \\
\hline 5 & 18 & 22 & 4 & 18 & 1362.0513 & -1 \\
\hline 5 & 19 & 22 & 4 & 19 & 1362.0716 & 6 \\
\hline 8 & 4 & 11 & 7 & 4 & 1362.1425 & -6 \\
\hline 4 & 24 & 26 & 3 & 24 & 1362.3187 & 20 \\
\hline 7 & 9 & 15 & 6 & 9 & 1362.7917 & 4 \\
\hline 9 & 0 & 8 & 8 & 0 & 1362.9418 & -25 \\
\hline 4 & 24 & 27 & 3 & 24 & 1363.0543 & -1 \\
\hline 6 & 14 & 19 & 5 & 14 & 1363.2971 & 4 \\
\hline 5 & 19 & 23 & 4 & 19 & 1363.5902 & 1 \\
\hline 5 & 20 & 23 & 4 & 20 & 1363.6135 & 8 \\
\hline 8 & 5 & 12 & 7 & 5 & 1363.7140 & -3 \\
\hline 7 & 10 & 16 & 6 & 10 & 1364.3589 & 8 \\
\hline 4 & 25 & 28 & 3 & 25 & 1364.4349 & 5 \\
\hline 9 & 1 & 9 & 8 & 1 & 1364.5152 & -17 \\
\hline 6 & 15 & 20 & 5 & 15 & 1364.8541 & 2 \\
\hline 5 & 20 & 24 & 4 & 20 & 1365.1217 & -13 \\
\hline 5 & 21 & 24 & 4 & 21 & 1365.1511 & 1 \\
\hline 8 & 6 & 13 & 7 & 6 & 1365.2850 & -1 \\
\hline 7 & 11 & 17 & 6 & 11 & 1365.9245 & 6 \\
\hline 9 & 2 & 10 & 8 & 2 & 1366.0883 & -15 \\
\hline 6 & 16 & 21 & 5 & 16 & 1366.4091 & 3 \\
\hline 5 & 21 & 25 & 4 & 21 & 1366.6488 & -11 \\
\hline 5 & 22 & 25 & 4 & 22 & 1366.6857 & 0 \\
\hline 8 & 7 & 14 & 7 & 7 & 1366.8561 & 4 \\
\hline 7 & 12 & 18 & 6 & 12 & 1367.4895 & 9 \\
\hline 9 & 3 & 11 & 8 & 3 & 1367.6617 & -11 \\
\hline 6 & 17 & 22 & 5 & 17 & 1367.9614 & 5 \\
\hline 5 & 22 & 26 & 4 & 22 & 1368.1681 & -22 \\
\hline 5 & 23 & 26 & 4 & 23 & 1368.2180 & 14 \\
\hline 8 & 8 & 15 & 7 & 8 & 1368.4263 & 5 \\
\hline 7 & 13 & 19 & 6 & 13 & 1369.0527 & 7 \\
\hline 9 & 4 & 12 & 8 & 4 & 1369.2355 & -4 \\
\hline 6 & 18 & 23 & 5 & 18 & 1369.5105 & 17 \\
\hline 5 & 23 & 27 & 4 & 23 & 1369.6818 & -20 \\
\hline 5 & 24 & 27 & 4 & 24 & 1369.7440 & 3 \\
\hline 10 & 0 & 9 & 9 & 0 & 1369.9230 & -35 \\
\hline 8 & 9 & 16 & 7 & 9 & 1369.9962 & 8 \\
\hline 7 & 14 & 20 & 6 & 14 & 1370.6149 & 9 \\
\hline 9 & 5 & 13 & 8 & 5 & 1370.8090 & 0 \\
\hline 6 & 20 & 24 & 5 & 20 & 1371.0509 & 0 \\
\hline 6 & 19 & 24 & 5 & 19 & 1371.0788 & 10 \\
\hline 5 & 24 & 28 & 4 & 24 & 1371.1858 & -37 \\
\hline 5 & 25 & 28 & 4 & 25 & 1371.2681 & 12 \\
\hline 10 & 1 & 10 & 9 & 1 & 1371.4984 & -27 \\
\hline 8 & 10 & 17 & 7 & 10 & 1371.5651 & 8 \\
\hline 7 & 15 & 21 & 6 & 15 & 1372.1752 & 7 \\
\hline 9 & 6 & 14 & 8 & 6 & 1372.3825 & 5 \\
\hline 6 & 20 & 25 & 5 & 20 & 1372.6079 & -12 \\
\hline 6 & 21 & 25 & 5 & 21 & 1372.6167 & 11 \\
\hline 5 & 25 & 29 & 4 & 25 & 1372.6837 & -29 \\
\hline 10 & 2 & 11 & 9 & 2 & 1373.0743 & -17 \\
\hline 8 & 11 & 18 & 7 & 11 & 1373.1336 & 12 \\
\hline 7 & 16 & 22 & 6 & 16 & 1373.7336 & 5 \\
\hline 9 & 7 & 15 & 8 & 7 & 1373.9555 & 8 \\
\hline 6 & 21 & 26 & 5 & 21 & 1374.1512 & 23 \\
\hline 10 & 3 & 12 & 9 & 3 & 1374.6506 & -6 \\
\hline 8 & 12 & 19 & 7 & 12 & 1374.7007 & 11 \\
\hline 7 & 17 & 23 & 6 & 17 & 1375.2903 & 4 \\
\hline 9 & 8 & 16 & 8 & 8 & 1375.5286 & 14 \\
\hline 6 & 22 & 27 & 5 & 22 & 1375.6886 & 25 \\
\hline 10 & 4 & 13 & 9 & 4 & 1376.2266 & 0 \\
\hline 8 & 13 & 20 & 7 & 13 & 1376.2670 & 13 \\
\hline 11 & 0 & 10 & 10 & 0 & 1376.8005 & -49 \\
\hline 7 & 18 & 24 & 6 & 18 & 1376.8442 & -3 \\
\hline 9 & 9 & 17 & 8 & 9 & 1377.1008 & 16 \\
\hline 6 & 23 & 28 & 5 & 23 & 1377.2201 & 3 \\
\hline 10 & 5 & 14 & 9 & 5 & 1377.8030 & 10 \\
\hline 8 & 14 & 21 & 7 & 14 & 1377.8316 & 10 \\
\hline 11 & 1 & 11 & 10 & 1 & 1378.3788 & -26 \\
\hline 7 & 19 & 25 & 6 & 19 & 1378.3969 & 1 \\
\hline 9 & 10 & 18 & 8 & 10 & 1378.6726 & 20 \\
\hline 6 & 24 & 29 & 5 & 24 & 1378.7491 & -3 \\
\hline
\end{tabular}




\begin{tabular}{|c|c|c|c|c|c|c|c|}
\hline 16 & 10 & 6 & 15 & 9 & 6 & 1379.3791 & 17 \\
\hline 27 & 7 & 20 & 26 & 6 & 20 & 1379.9461 & -5 \\
\hline 13 & 11 & 2 & 12 & 10 & 2 & 1379.9572 & -5 \\
\hline 20 & 9 & 11 & 19 & 8 & 11 & 1380.2432 & 18 \\
\hline 31 & 6 & 25 & 30 & 5 & 25 & 1380.2736 & -10 \\
\hline 24 & 8 & 16 & 23 & 7 & 16 & 1380.9556 & -7 \\
\hline 17 & 10 & 7 & 16 & 9 & 7 & 1380.9556 & 30 \\
\hline 28 & 7 & 21 & 27 & 6 & 21 & 1381.4919 & -17 \\
\hline 14 & 11 & 3 & 13 & 10 & 3 & 1381.5376 & 35 \\
\hline 21 & 9 & 12 & 20 & 8 & 12 & 1381.8135 & 21 \\
\hline 25 & 8 & 17 & 24 & 7 & 17 & 1382.5168 & 1 \\
\hline 18 & 10 & 8 & 17 & 9 & 8 & 1382.5303 & 28 \\
\hline 29 & 7 & 22 & 28 & 6 & 22 & 1383.0354 & -22 \\
\hline 22 & 9 & 13 & 21 & 8 & 13 & 1383.3832 & 27 \\
\hline 26 & 8 & 18 & 25 & 7 & 18 & 1384.0721 & -32 \\
\hline 19 & 10 & 9 & 18 & 9 & 9 & 1384.1050 & 30 \\
\hline 30 & 7 & 23 & 29 & 6 & 23 & 1384.5750 & -34 \\
\hline 16 & 11 & 5 & 15 & 10 & 5 & 1384.6846 & 0 \\
\hline 23 & 9 & 14 & 22 & 8 & 14 & 1384.9492 & 7 \\
\hline 20 & 10 & 10 & 19 & 9 & 10 & 1385.6788 & 28 \\
\hline 17 & 11 & 6 & 16 & 10 & 6 & 1386.2610 & 17 \\
\hline 24 & 9 & 15 & 23 & 8 & 15 & 1386.5148 & -5 \\
\hline 28 & 8 & 20 & 27 & 7 & 20 & 1387.1839 & -26 \\
\hline 21 & 10 & 11 & 20 & 9 & 11 & 1387.2514 & 21 \\
\hline 32 & 7 & 25 & 31 & 6 & 25 & 1387.6427 & -64 \\
\hline 18 & 11 & 7 & 17 & 10 & 7 & 1387.8335 & 8 \\
\hline 25 & 9 & 16 & 24 & 8 & 16 & 1388.0790 & -18 \\
\hline 29 & 8 & 21 & 28 & 7 & 21 & 1388.7343 & -43 \\
\hline 22 & 10 & 12 & 21 & 9 & 12 & 1388.8220 & 2 \\
\hline 19 & 11 & 8 & 18 & 10 & 8 & 1389.4030 & -12 \\
\hline 26 & 9 & 17 & 25 & 8 & 17 & 1389.6415 & -32 \\
\hline 20 & 11 & 9 & 19 & 10 & 9 & 1390.9666 & -63 \\
\hline 27 & 9 & 18 & 26 & 8 & 18 & 1391.2018 & -53 \\
\hline 24 & 10 & 14 & 23 & 9 & 14 & 1391.9589 & -47 \\
\hline
\end{tabular}

v9 Band

$\begin{array}{rrrr}24 & 6 & 18 & 25 \\ 23 & 6 & 17 & 24 \\ 22 & 6 & 16 & 23 \\ 22 & 8 & 14 & 23 \\ 21 & 6 & 15 & 22 \\ 21 & 7 & 14 & 22 \\ 21 & 8 & 13 & 22 \\ 20 & 5 & 15 & 21 \\ 20 & 6 & 14 & 21 \\ 20 & 7 & 13 & 21 \\ 20 & 8 & 12 & 21 \\ 20 & 9 & 11 & 21 \\ 19 & 6 & 13 & 20 \\ 19 & 7 & 12 & 20 \\ 18 & 5 & 13 & 19 \\ 19 & 9 & 10 & 20 \\ 18 & 6 & 12 & 19 \\ 18 & 7 & 11 & 19 \\ 18 & 8 & 10 & 19 \\ 17 & 5 & 12 & 18 \\ 18 & 9 & 9 & 19 \\ 17 & 6 & 11 & 18 \\ 17 & 7 & 10 & 18 \\ 17 & 8 & 9 & 18 \\ 16 & 5 & 11 & 17 \\ 17 & 9 & 8 & 18 \\ 16 & 6 & 10 & 17 \\ 16 & 7 & 9 & 17 \\ 16 & 8 & 8 & 17 \\ 15 & 4 & 11 & 16 \\ 16 & 9 & 7 & 17 \\ 15 & 5 & 10 & 16 \\ 15 & 6 & 9 & 16 \\ 16 & 10 & 6 & 17 \\ 15 & 7 & 8 & 16 \\ 15 & 8 & 7 & 16 \\ 14 & 4 & 10 & 15 \\ 15 & 9 & 6 & 16 \\ 14 & 5 & 9 & 15 \\ 14 & 6 & 8 & 15\end{array}$

$\begin{array}{rrrrrrr}9 & & & & 5 & 4 \\ 6 & 20 & 1093.9551 & 26 & 7 & 8 & 5 \\ 6 & 19 & 1095.5764 & 12 & 7 & 7 & 5 \\ 6 & 18 & 1097.1928 & 7 & 7 & 6 & 5 \\ 8 & 16 & 1098.1506 & -3 & 7 & 5 & 5 \\ 6 & 17 & 1098.8039 & -1 & 7 & 10 & 6 \\ 7 & 16 & 1099.2314 & -15 & 7 & 9 & 6 \\ 8 & 15 & 1099.7337 & -6 & 7 & 8 & 6 \\ 5 & 17 & 1100.1536 & 14 & 7 & 7 & 6 \\ 6 & 16 & 1100.4110 & -5 & 7 & 6 & 6 \\ 7 & 15 & 1100.8264 & -7 & 7 & 10 & 7 \\ 8 & 14 & 1101.3108 & -36 & 7 & 9 & 7 \\ 9 & 13 & 1101.9029 & -8 & 10 & 8 & 7 \\ 6 & 15 & 1102.0137 & -13 & 7 & 7 & 7 \\ 7 & 14 & 1102.4180 & -1 & 7 & 9 & 8 \\ 5 & 15 & 1103.3443 & -1 & 7 & 8 & 8 \\ 9 & 12 & 1103.4599 & -11 & 7 & 11 & 8 \\ 6 & 14 & 1103.6147 & -1 & 7 & 13 & 8 \\ 7 & 13 & 1104.0056 & -9 & 7 & 12 & 8 \\ 8 & 12 & 1104.4658 & -1 & 10 & 10 & 8 \\ 5 & 14 & 1104.9379 & 1 & 7 & 9 & 9 \\ 9 & 11 & 1105.0147 & -1 & 7 & 10 & 9 \\ 6 & 13 & 1105.2112 & -3 & 7 & 11 & 9 \\ 7 & 12 & 1105.5908 & -14 & 7 & 12 & 9 \\ 8 & 11 & 1106.0348 & -30 & 10 & 13 & 9 \\ 5 & 13 & 1106.5300 & 5 & 7 & 14 & 9 \\ 9 & 10 & 1106.5647 & -10 & 7 & 15 & 9 \\ 6 & 12 & 1106.8026 & -26 & 10 & 10 & 10 \\ 7 & 11 & 1107.1743 & -13 & 7 & 11 & 10 \\ 8 & 10 & 1107.6060 & -14 & 10 & 12 & 10 \\ 4 & 13 & 1107.8662 & 0 & 7 & 13 & 10 \\ 9 & 9 & 1108.1135 & -6 & 10 & 14 & 10 \\ 5 & 12 & 1108.1200 & 4 & 7 & 15 & 10 \\ 6 & 11 & 1108.3958 & -5 & 7 & 6 & 4 \\ 10 & 8 & 1108.7248 & 22 & 10 & 6 & 5 \\ 7 & 10 & 1108.7575 & 7 & 7 & 7 & 4 \\ 8 & 9 & 1109.1758 & 7 & 7 & 7 & 5 \\ 4 & 12 & 1109.4603 & 6 & 7 & 7 & 6 \\ 9 & 8 & 1109.6598 & -8 & 7 & 8 & 4 \\ 5 & 11 & 1109.7087 & 6 & 7 & 5 & 6 \\ 6 & 10 & 1109.9843 & -6 & 7 & & \end{array}$

$\begin{array}{lll}1110.2323 & 3 & 7\end{array}$

$\begin{array}{rrr}1110.3351 & -9 & 7 \\ 1111.0521 & 3 & 7\end{array}$

$\begin{array}{lll}1111.2076 & 21 & 7\end{array}$

$\begin{array}{lll}1111.5705 & -8 & 7\end{array}$

$\begin{array}{rrr}1111.7401 & 7 & 7\end{array}$

$\begin{array}{lll}1111.9117 & -18 & 7\end{array}$

$\begin{array}{rrr}1112.6417 & -6 & 10\end{array}$

$\begin{array}{lll}1112.8806 & -3 & 7\end{array}$

$1113.1568 \quad 127$

$\begin{array}{lll}1113.4890 & -4 & 7\end{array}$

$1114.2312 \quad-1 \quad 7$

$\begin{array}{lll}1114.2923 & -1 & 7\end{array}$

$\begin{array}{lll}1114.4655 & 2 & 7\end{array}$

$1114.7382 \quad 27$

$\begin{array}{lll}1115.0635 & -4 & 7\end{array}$

$\begin{array}{rrr}1115.4315 & -3 & 7 \\ 1115.8353 & 1 & 7\end{array}$

$\begin{array}{rrr}1115.8353 & 1 & 7 \\ 1116.0490 & 7 & 10\end{array}$

$\begin{array}{lll}1116.3188 & 1 & 7\end{array}$

$\begin{array}{lll}1116.6371 & -1 & 7\end{array}$

$\begin{array}{lll}1116.9935 & -3 & 7\end{array}$

$\begin{array}{lll}1117.4036 & -8 & 7\end{array}$

$\begin{array}{lll}1117.6307 & 6\end{array}$

$1117.8981 \quad 3 \quad 7$

$\begin{array}{rrr}1118.2097 & 4 & 7\end{array}$

$\begin{array}{rrr}1118.5554 & 1 & 10 \\ 1118.9893 & 9 & 7\end{array}$

$\begin{array}{lll}1119.2107 & 0 & 7\end{array}$

$\begin{array}{lll}1119.4760 & 6 & 7\end{array}$

$\begin{array}{lll}1119.7808 & 2 & 7\end{array}$

$\begin{array}{rrr}1120.5719 & 12 & 7\end{array}$

$1120.7905 \quad 5 \quad 7$

$\begin{array}{lll}1121.0524 & 8 & 7\end{array}$

$1121.3529 \quad 19 \quad 7$

$\begin{array}{rrr}1122.1518 & 6 & 7 \\ 1123.7310 & 10 & 7\end{array}$

$\begin{array}{lll}1133.3151 & 18 & 7\end{array}$

$1133.3247-16 \quad 7$

$\begin{array}{rr}1133.3379 & 2\end{array}$

$\begin{array}{rrr}1133.3482 & 5 & 7\end{array}$

$\begin{array}{lll}1133.5495 & -2 & 7\end{array}$

$\begin{array}{lll}1133.5625 & -9 & 7\end{array}$

$\begin{array}{lll}1133.5748 & -5 & 7\end{array}$

$\begin{array}{lll}1133.5863 & 8 & 7\end{array}$

$\begin{array}{lll}1133.5945 & 3 & 7\end{array}$

$1133.8644 \quad 17$

$\begin{array}{lll}1133.8714 & -1 & 7\end{array}$

$\begin{array}{rrr}1133.8781 & 6 & 7 \\ 1133.8837 & 13 & 7\end{array}$

$\begin{array}{lll}1134.2123 & -16 & 10\end{array}$

$\begin{array}{lrr}1134.2123 & 19 & 10\end{array}$

$\begin{array}{lll}1134.2174 & -17 & 10\end{array}$

$\begin{array}{lll}1134.2174 & -26 & 10\end{array}$

$\begin{array}{lll}1134.2174 & -28 & 10\end{array}$

$\begin{array}{lr}1134.2174 \quad 5 & 10\end{array}$

$\begin{array}{lll}1134.5770 & 7 & 7\end{array}$

$\begin{array}{lll}1134.5976 & 8 & 7\end{array}$

$\begin{array}{lll}1134.6176 & 1 & 7\end{array}$

$\begin{array}{lll}1134.6378 & -1 & 7\end{array}$

$\begin{array}{lll}1134.6550 & -26 & 7\end{array}$

$1134.6762 \quad 1 \quad 10$

$1134.6946 \quad 16 \quad 10$

$\begin{array}{rrr}1134.9793 & 12 & 7\end{array}$

$\begin{array}{lll}1135.0353 & 11 & 7\end{array}$

$\begin{array}{lll}1135.0920 & 10 & 7\end{array}$

$\begin{array}{lll}1135.1483 & 8 & 7\end{array}$

$\begin{array}{lll}1135.2044 & 13 & 7\end{array}$

$\begin{array}{rrr}1135.2588 & 19 & 10\end{array}$

$1142.5276 \quad 3 \quad 7$

$1142.7406 \quad-1 \quad 10$

$\begin{array}{lll}1144.0822 & 13 & 10\end{array}$

$\begin{array}{lll}1144.2953 & -7 & 10\end{array}$

$\begin{array}{lll}1144.5547 & 14 & 7\end{array}$

$\begin{array}{lll}1145.6317 & -8 & 7\end{array}$

$\begin{array}{lll}1145.8501 & 5 & 7 \\ 1146.1097 & 5 & 7\end{array}$ 


\begin{tabular}{|c|c|c|c|c|c|c|c|c|}
\hline 8 & 7 & 1 & 7 & 7 & 1 & 1146.4078 & -11 & 10 \\
\hline 9 & 4 & 5 & 8 & 4 & 5 & 1147.1818 & -1 & 7 \\
\hline 9 & 5 & 4 & 8 & 5 & 4 & 1147.4019 & 3 & \\
\hline 9 & 6 & 3 & 8 & 6 & 3 & 1147.6633 & 0 & \\
\hline 9 & 7 & 2 & 8 & 7 & 2 & 1147.9682 & -3 & \\
\hline 10 & 4 & 6 & 9 & 4 & 6 & 1148.7296 & 3 & \\
\hline 10 & 5 & 5 & 9 & 5 & 5 & 1148.9518 & 0 & \\
\hline 10 & 6 & 4 & 9 & 6 & 4 & 1149.2149 & -4 & \\
\hline 10 & 7 & 3 & 9 & 7 & 3 & 1149.5266 & 1 & 0 \\
\hline 10 & 8 & 2 & 9 & 8 & 2 & 1149.8758 & 4 & \\
\hline 11 & 4 & 7 & 10 & 4 & 7 & 1150.2750 & 3 & \\
\hline 11 & 5 & 6 & 10 & 5 & 6 & 1150.4998 & -3 & \\
\hline 11 & 6 & 5 & 10 & 6 & 5 & 1150.7645 & -5 & \\
\hline 11 & 7 & 4 & 10 & 7 & 4 & 1151.0826 & -2 & \\
\hline 11 & 8 & 3 & 10 & 8 & 3 & 1151.4419 & -2 & \\
\hline 12 & 4 & 8 & 11 & 4 & 8 & 1151.8180 & 0 & \\
\hline 11 & 9 & 2 & 10 & 9 & 2 & 1151.8367 & 7 & \\
\hline 12 & 5 & 7 & 11 & 5 & 7 & 1152.0469 & 3 & \\
\hline 12 & 6 & 6 & 11 & 6 & 6 & 1152.3119 & -4 & \\
\hline 12 & 7 & 5 & 11 & 7 & 5 & 1152.6367 & -4 & \\
\hline 12 & 8 & 4 & 11 & 8 & 4 & 1153.0066 & -8 & \\
\hline 13 & 4 & 9 & 12 & 4 & 9 & 1153.3590 & -3 & \\
\hline 12 & 9 & 3 & 11 & 9 & 3 & 1153.4199 & -2 & \\
\hline 13 & 5 & 8 & 12 & 5 & 8 & 1153.5911 & 1 & \\
\hline 13 & 6 & 7 & 12 & 6 & 7 & 1153.8565 & -4 & , \\
\hline 12 & 10 & 2 & 11 & 10 & 2 & 1153.8681 & 3 & 10 \\
\hline 13 & 7 & 6 & 12 & 7 & 6 & 1154.1897 & 5 & 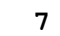 \\
\hline 13 & 8 & 5 & 12 & 8 & 5 & 1154.5698 & -12 & \\
\hline 14 & 4 & 10 & 13 & 4 & 10 & 1154.8982 & -3 & 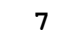 \\
\hline 13 & 9 & 4 & 12 & 9 & 4 & 1155.0030 & -1 & \\
\hline 14 & 5 & 9 & 13 & 5 & 9 & 1155.1340 & 7 & 7 \\
\hline 14 & 6 & 8 & 13 & 6 & 8 & 1155.3980 & -7 & \\
\hline 13 & 10 & 3 & 12 & 10 & 3 & 1155.4879 & 7 & \\
\hline 14 & 7 & 7 & 13 & 7 & 7 & 1155.7385 & -3 & 7 \\
\hline 14 & 8 & 6 & 13 & 8 & 6 & 1156.1318 & -6 & , \\
\hline 15 & 4 & 11 & 14 & 4 & 11 & 1156.4344 & -12 & \\
\hline 14 & 9 & 5 & 13 & 9 & 5 & 1156.5839 & -5 & 7 \\
\hline 15 & 5 & 10 & 14 & 5 & 10 & 1156.6741 & 8 & 7 \\
\hline 15 & 6 & 9 & 14 & 6 & 9 & 1156.9364 & -9 & \\
\hline 14 & 10 & 4 & 13 & 10 & 4 & 1157.1068 & 18 & \\
\hline 15 & 7 & 8 & 14 & 7 & 8 & 1157.2847 & -9 & \\
\hline 15 & 8 & 7 & 14 & 8 & 7 & 1157.6908 & -7 & 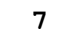 \\
\hline 15 & 9 & 6 & 14 & 9 & 6 & 1158.1635 & -1 & \\
\hline 16 & 5 & 11 & 15 & 5 & 11 & 1158.2116 & 6 & \\
\hline 16 & 6 & 10 & 15 & 6 & 10 & 1158.4719 & -6 & 7 \\
\hline 15 & 10 & 5 & 14 & 10 & 5 & 1158.7222 & 16 & \\
\hline 16 & 7 & 9 & 15 & 7 & 9 & 1158.8287 & -8 & \\
\hline 16 & 8 & 8 & 15 & 8 & 8 & 1159.2466 & -12 & 7 \\
\hline 16 & 9 & 7 & 15 & 9 & 7 & 1159.7395 & -7 & \\
\hline 17 & 5 & 12 & 16 & 5 & 12 & 1159.7473 & 11 & \\
\hline 17 & 6 & 11 & 16 & 6 & 11 & 1160.0046 & 5 & \\
\hline 16 & 10 & 6 & 15 & 10 & 6 & 1160.3356 & 23 & \\
\hline 17 & 7 & 10 & 16 & 7 & 10 & 1160.3694 & -8 & \\
\hline 17 & 8 & 9 & 16 & 8 & 9 & 1160.7999 & -11 & \\
\hline 18 & 5 & 13 & 17 & 5 & 13 & 1161.2797 & 9 & 7 \\
\hline 17 & 9 & 8 & 16 & 9 & 8 & 1161.3134 & -1 & \\
\hline 18 & 6 & 12 & 17 & 6 & 12 & 1161.5309 & -7 & \\
\hline 18 & 7 & 11 & 17 & 7 & 11 & 1161.9056 & -17 & 7 \\
\hline 17 & 10 & 7 & 16 & 10 & 7 & 1161.9435 & 13 & \\
\hline 18 & 8 & 10 & 17 & 8 & 10 & 1162.3497 & -11 & \\
\hline 19 & 5 & 14 & 18 & 5 & 14 & 1162.8097 & 11 & 7 \\
\hline 18 & 9 & 9 & 17 & 9 & 9 & 1162.8829 & -3 & \\
\hline 19 & 6 & 13 & 18 & 6 & 13 & 1163.0545 & -3 & \\
\hline 19 & 7 & 12 & 18 & 7 & 12 & 1163.4401 & -5 & \\
\hline 18 & 10 & 8 & 17 & 10 & 8 & 1163.5482 & 14 & 7 \\
\hline 19 & 8 & 11 & 18 & 8 & 11 & 1163.8959 & -10 & \\
\hline 20 & 5 & 15 & 19 & 5 & 15 & 1164.3374 & 18 & \\
\hline 19 & 9 & 10 & 18 & 9 & 10 & 1164.4464 & -22 & 10 \\
\hline 20 & 6 & 14 & 19 & 6 & 14 & 1164.5728 & -3 & \\
\hline 20 & 7 & 13 & 19 & 7 & 13 & 1164.9689 & -9 & \\
\hline 19 & 10 & 9 & 18 & 10 & 9 & 1165.1474 & 10 & 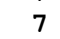 \\
\hline 20 & 8 & 12 & 19 & 8 & 12 & 1165.4379 & -9 & \\
\hline 21 & 5 & 16 & 20 & 5 & 16 & 1165.8606 & 11 & \\
\hline 20 & 9 & 11 & 19 & 9 & 11 & 1166.0078 & -15 & \\
\hline 21 & 6 & 15 & 20 & 6 & 15 & 1166.0862 & -1 & \\
\hline 21 & 7 & 14 & 20 & 7 & 14 & 1166.4941 & -5 & \\
\hline 20 & 10 & 10 & 19 & 10 & 10 & 1166.7416 & 11 & \\
\hline
\end{tabular}

